\title{
Design \\ of \\ Support Structures \\ for \\ Offshore Wind Turbines
}

\section{Proefschrift}

ter verkrijging van de graad van doctor aan de Technische Universiteit Delft,

op gezag van de Rector Magnificus prof. dr. ir. J.T. Fokkema in het openbaar te verdedigen ten overstaan van een commissie, door het College voor Promoties aangewezen, op woensdag 26 april 2006 te 13:00 uur door

Jan VAN DER TEMPEL

Civiel ingenieur geboren te Enschede 
Dit proefschrift is goedgekeurd door de promotoren:

Prof. dr. ir. J.H. Vugts

Prof. dr. ir. G.A.M. van Kuik

Samenstelling promotiecommissie:

Rector Magnificus

Prof. dr. ir. J.H. Vugts

Prof. dr. ir. G.A.M. van Kuik

Prof. ir. J. Meek

Prof. dr. ir. M.J.L. van Tooren

Prof. dr. dipl-ing. M.J. Kühn

Ir. E. Berkel

D.C. Quarton, M.A. C.Eng. voorzitter

Technische Universiteit Delft, promotor

Technische Universiteit Delft, promotor

Technische Universiteit Delft

Technische Universiteit Delft

Universität Stuttgart

Shell WindEnergy, Den Haag

Garrad Hassan \& Partners, Bristol

This $\mathrm{PhD}$ thesis was sponsored by the Cornelis Lely Stichting.

ISBN 90-76468-11-7

Offshore Engineering, report 2006.OE.009

DUWIND, report 2006.029

Published and distributed by the author in cooperation with:

Offshore Engineering
Stevinweg 1
2628 CN Delft
+31152784758
www.offshore.tudelft.nl

Offshore Engineering

$2628 \mathrm{CN}$ Delft

www.offshore.tudelft.nl
DUWIND

Kluyverweg 1

2629 HS Delft

+31 152785170

www.duwind.tudelft.nl

Cover picture: $\quad$ Courtesy of Gunnar Britse $@$ www.windpowerphotos.com

Printing: $\quad$ FEBO Druk B.V., Enschede

Copyright (C) 2006 Jan van der Tempel

No part of this book may be reproduced in any form, by print, copy

or in any other way without prior written permission from the author. 


\section{Acknowledgements}

Many people and institutions have contributed directly and indirectly to this thesis. I would like to thank them all very sincerely and name (too) few. First and foremost Prof. dr. ir. Jan Vugts for sending me an e-mail in the spring of 2001 to ask me to apply for this $\mathrm{PhD}$ position. Jan, you were very consistent in stating that, though being a hard worker, I most certainly am not the typical academic scholar. I still am not, though I tried to change the definition of typical. Our differences in approach always converged at some point and I must say I see your strict ways of working emerge in my own methods more and more. Prof. dr. ir. Gijs van Kuik I would like to thank for offering an inspiring working environment in the Section for Wind Energy and DUWIND with the numerous projects and extra-curricular activities I was allowed and encouraged to participate in. Thanks to Prof. ir. Jan Meek for his personal coaching and trust and for creating an atmosphere of professional efficiency which has become a true centre of excellence in offshore engineering.

Of over 50 students I had the privilege to supervise, the contributions of David Cerda Salzmann and Wybren de Vries to this thesis are the most pronounced and I am very happy that they help set a new standard for $\mathrm{PhD}$ people and content. Furthermore the scientific assistance of Toni Subroto and Michiel Zaaijer is gratefully acknowledged. Hans Mulder and Ruud van Rooij are thanked for providing the insights in the greater world of university systems and subsidy grants; Joke and Sylvia for always knowing a way around bureaucratic obstacles. David-Pieter Molenaar is thanked for sharing that beer in Berlin that gave us the idea for the Ampelmann.

Outside the university, the following companies are acknowledged: Shell WindEnergy, Ballast Nedam, Heerema, Argoss and Rambøll for providing valuable data and documents. Tim Camp and Mungo Morris of Garrad Hassan \& Partners are acknowledged for our joint efforts in the OWTES project and their support with Bladed. Furthermore, Jos Beurskens and Chris Westra are thanked for having set up the national offshore wind energy consortium We@Sea through which this work will be disseminated.

This PhD work was supported by a grant from the Cornelis Lely Stichting. 


\section{Summary}

To meet growing energy demands, the Kyoto protocol and the much desired diversification of supply, wind energy has become a mainstream source of energy in the EU. Cost wise it is already competing with gas fired electricity. In the last decade wind moved offshore to accommodate even more wind power. The offshore wind resource is more abundant and of a better quality, resulting in higher electricity output. On the other hand, the cost of installing turbines offshore is higher than onshore.

To improve the cost-effectiveness of offshore wind, the risks involved must be known and mitigated and the critical design parameters must be optimised. From an engineering point of view, these requirements can be met through the following steps:

- understand the basics of offshore wind turbines

- apply lessons learned from previous projects

- improve design tools.

This thesis focuses on the design of the support structure. First, the basics of offshore engineering and of wind energy technology are summarized, specifically focused on the support structure design. Then, an overview is given of four actual offshore wind farm designs and their details. The design methods were compared mutually and with a design of a typical offshore oil platform. For most of the design steps, the methodology is consistent. Only the fatigue damage assessment is done differently for each individual project.

Fatigue assessment in offshore engineering is done in the frequency domain. This method can be applied because the wave loads can be effectively linearized. The advantages of the frequency domain method are the clarity of presentation of intermediate results and the final outcome as well as the speed of calculation. The offshore wind industry standard (both onshore and offshore) is to use time domain simulations, which enables taking all non-linearities of the turbine operation into account. A disadvantage of this for the design of support structures is that offshore contractors lack both the aerodynamic knowledge and knowledge of the turbine details to use the full time domain simulation method to calculate the total fatigue damage. In this thesis a frequency domain method is developed to solve this problem. An interface between turbine manufacturer and offshore contractor is created that avoids the need to transfer commercially sensitive turbine details. The offshore contractor can further optimise the support structures with the software packages he normally uses.

The frequency domain method is tested for the Blyth offshore wind turbines, for which a validated computer model and on-site measurements were available. Further, the 
method is applied to a design for the Dutch offshore wind farm to be erected at Egmond in 2006. In both cases, the frequency domain method works very well and gives results that compare well with time domain results. The computer time required to perform a fatigue calculation has been reduced from several hours in the time domain to less than 2 minutes in the frequency domain. This high speed of calculation opens possibilities for parameter variations to check the sensitivity of design choices and for optimisation of every structure within the wind farm. This has the potential to significantly reduce cost and risk.

A key issue in the accuracy of the method is the effect of the aerodynamic damping of the operating turbine on support structure dynamics. Several calculation methods for this damping have been tested and have shown to give reasonable results. More work is needed to more accurately pinpoint the magnitude of this aerodynamic damping.

The frequency domain method is currently being implemented in the software of an offshore contractor while other companies have already shown interest. 


\section{Table of contents}

Acknowledgements.....................................................................................................................

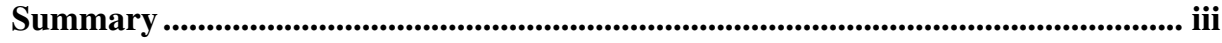

Table of contents.............................................................................................................................

List of symbols ............................................................................................................................. vii

1.Introduction....................................................................................................................................

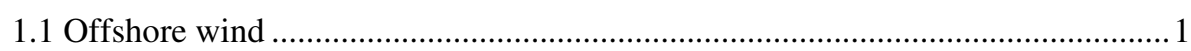

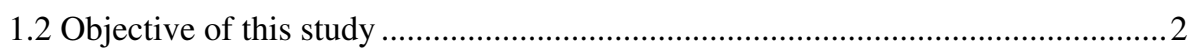

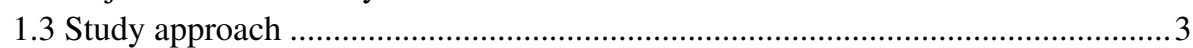

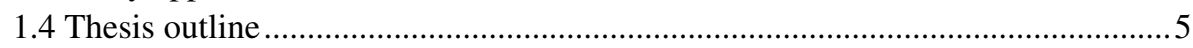

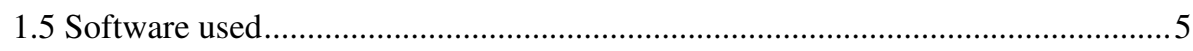

2. Basics of offshore, wind and turbines ........................................................................

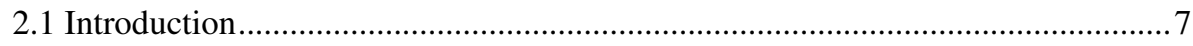

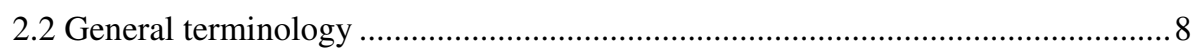

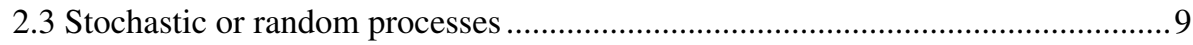

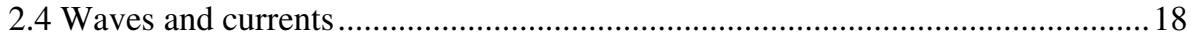

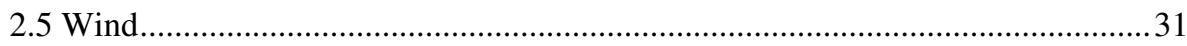

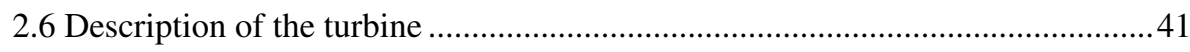

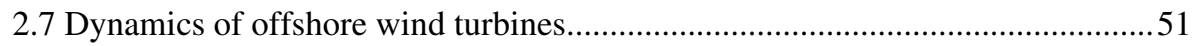

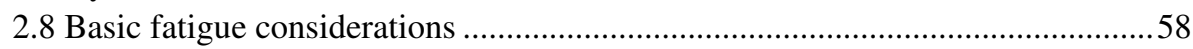

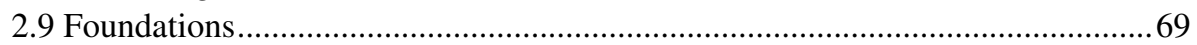

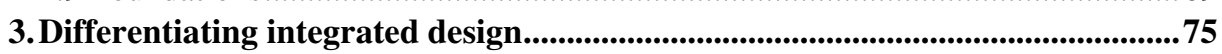

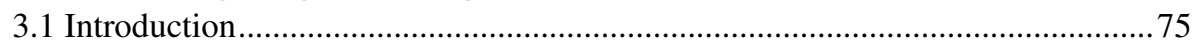

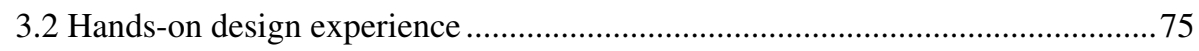

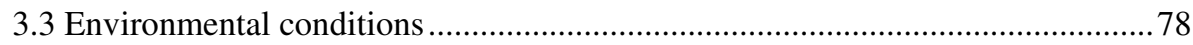

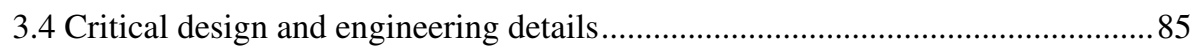

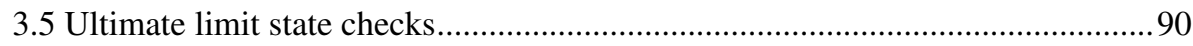

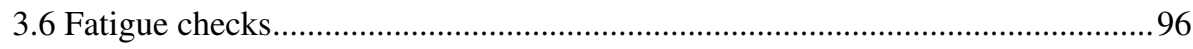

3.7 More options for fatigue: frequency domain ................................................... 102

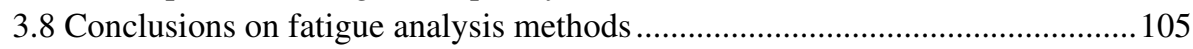

4.Frequency domain fatigue due to waves...................................................................107

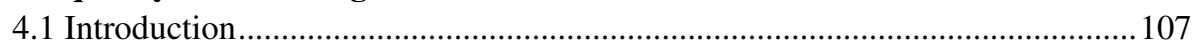

4.2 Basics of frequency domain calculations: the 1 DOF system ..............................107

4.3 Example application to an offshore wind turbine.............................................111

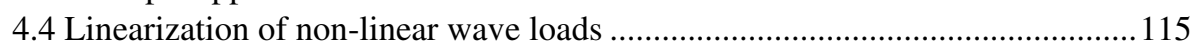

4.5 Frequency domain determination of fatigue damage due to waves ...................119

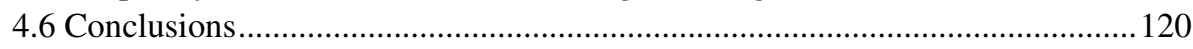


5.Devising a frequency domain method for offshore wind turbine fatigue..........121

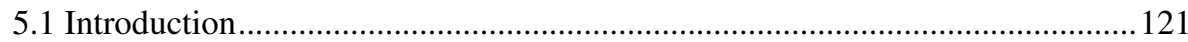

5.2 Similarities between time and frequency domain approaches............................121

5.3 Determining stress response spectra under wind loading.................................. 124

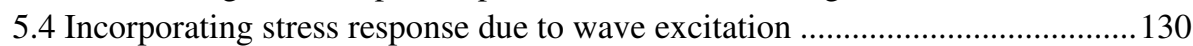

5.5 Fatigue damage calculation using the spectra of the total stress response .........130

5.6 Expert opinions on fatigue damage check in the time domain........................... 132

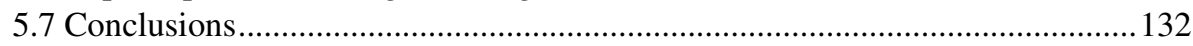

6. Application of frequency domain fatigue to Blyth ........................................135

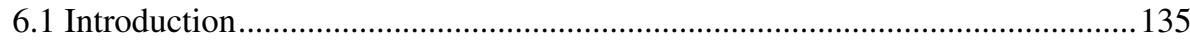

6.2 Description of the simulation model and parameter settings ..............................135

6.3 Transfer function for fluctuating wind speed to tower top load ........................143

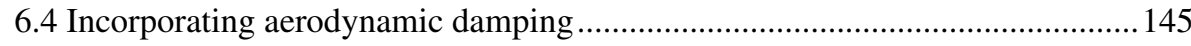

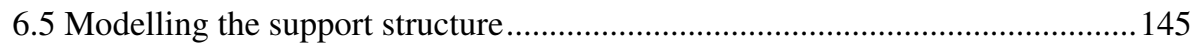

6.6 Comparison of frequency domain calculations with time domain calculations and with measurements under wind loading only ................................................ 148

6.7 Comparison of frequency domain results with time domain and measurements under combined wind and wave loading............................................................ 151

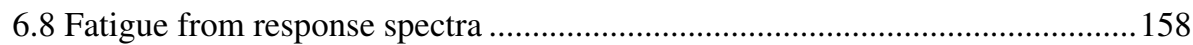

6.9 Conclusions on frequency domain fatigue calculations .................................... 163

7.Frequency domain fatigue for OWEZ ...................................................165

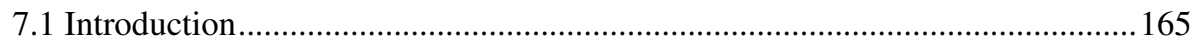

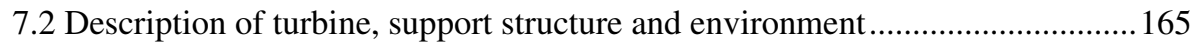

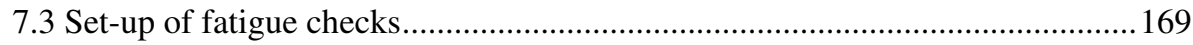

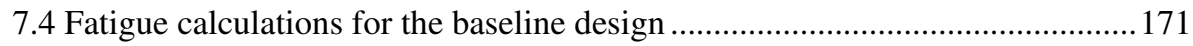

7.5 The effect of scour on the lifetime fatigue damage ...................................... 178

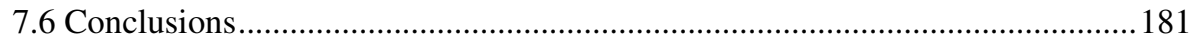

8. Conclusions and Outlook ...................................................................................... 183

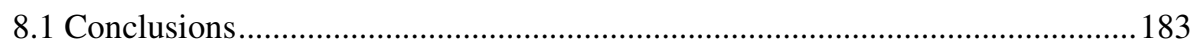

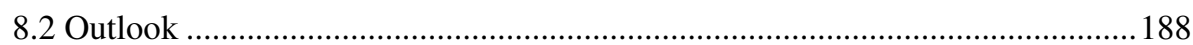

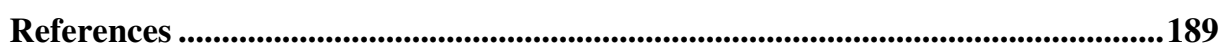

Appendix I Wave induced inertia and drag load and moment graphs.................195

Appendix II 3D scatter diagram for OWEZ site.......................................................197

Appendix III Parameters of all 112 states .........................................................200

Appendix IV Comparison of response spectra for time domain and frequency

domain for 112 states for manually fitted aerodynamic damping ....................201

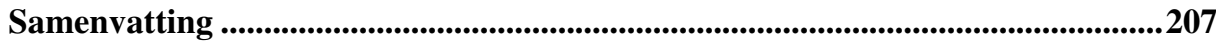

Curriculum vitae .........................................................................................................209 


\section{List of symbols}

$\begin{array}{ll}1 \mathrm{P} & \text { rotation frequency of turbine } \\ 3 \mathrm{P} & \text { blade passing frequency of three-bladed turbine } \\ a & \text { induction factor } \\ A & \text { exposed area } \\ A_{0} & \text { mean of a signal } \\ A_{\text {disk }} & \text { area of the actuator disk } \\ A_{F} & \text { factor in Morison equation calculation } \\ A_{M} & \text { factor in Morison equation calculation } \\ A_{q} & \text { Fourier coefficient } \\ A_{\text {rotor }} & \text { swept area of the rotor } \\ a_{s i} & \text { added mass coefficient of impacting ship } \\ A_{\text {wave }} & \text { wave amplitude } \\ B_{F} & \text { factor in Morison equation calculation } \\ B_{M} & \text { factor in Morison equation calculation } \\ B_{q} & \text { Fourier coefficient } \\ c & \text { damping coefficient } \\ c_{a} & \text { airfoil chord length } \\ c_{a}(r) & \text { airfoil chord length at radial position } r \\ C_{\text {aero }} & \text { aerodynamic coefficient } \\ c_{\text {critical }} & \text { critical damping } \\ C_{d} & \text { hydrodynamic drag coefficient } \\ C_{D}(\alpha) & \text { aerodynamic drag coefficient } \\ c_{d a m p i n g} & \text { damping coefficient } \\ C_{L}(\alpha) & \text { tower bending stiffness } \\ C_{m} & \text { aerodynamic lift coefficient } \\ C_{P} & \text { hydrodynamic inertia coefficient } \\ C_{s} & \text { power coefficient } \\ c_{s i} & \text { slamming coefficient } \\ c_{w} & \text { stiffness of impacting part of vessel } \\ d & \text { Weibull scale parameter } \\ D & \text { water depth } \\ D_{a v} & \text { cylinder diameter } \\ D_{\text {fat }} & \text { tower average diameter }=D-t_{w} \\ D_{\text {fat,life }} & \text { fatigue damage ratio per unit of time } \\ D_{\text {fat,state }} & \text { lifetime fatigue damage } \\ D_{r} & \text { fatigue damage per environmental state } \\ D_{\text {rotor }} & E I \\ E & \text { relative density of sand } \\ f & \end{array}$

[Hz]

$[\mathrm{Hz}]$

[-]

[m $\left.{ }^{2}\right]$

[...]

$\left[\mathrm{m}^{2}\right]$

$[-]$

[-]

[-]

$\left[\mathrm{m}^{2}\right]$

[-]

[m]

$[-]$

$[-]$

[-]

[Ns/m]

[m]

[m]

[-]

[Ns/m]

[-]

[-]

$[\mathrm{Ns} / \mathrm{m}]$

[-]

[-]

[-]

$[-]$

[N/m]

[-]

[m]

[m]

[m]

[1/time]

[-]

[-]

$\left[\mathrm{kg} / \mathrm{m}^{3}\right]$

[m]

$\left[\mathrm{N} / \mathrm{m}^{2}\right]$

$\left[\mathrm{Nm}^{2}\right]$

[Hz] 


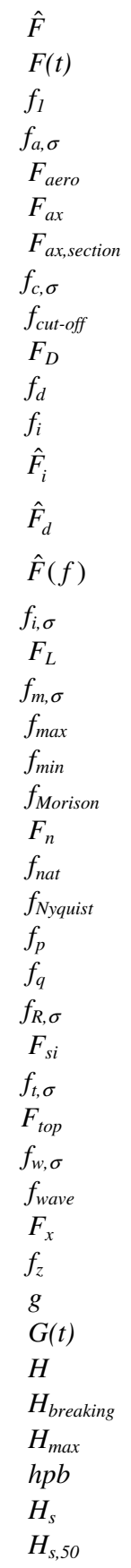

amplitude of excitation load

load as function of time

basic frequency, $1 / T$

$[\mathrm{Hz}]$

effect of misalignment

$[-]$

aerodynamic load

$[\mathrm{N}]$

axial load on actuator disk

$[\mathrm{N}]$

axial load on blade section $\quad[\mathrm{N}]$

corrosion effect

cut-off frequency $[\mathrm{Hz}]$

aerodynamic drag load $\quad[\mathrm{N}]$

hydrodynamic drag load per unit length $\quad[\mathrm{N} / \mathrm{m}]$

hydrodynamic inertia load per unit length $\quad[\mathrm{N} / \mathrm{m}]$

amplitude of hydrodynamic inertia load $[\mathrm{N}]$

amplitude of hydrodynamic drag load [N]

amplitude of excitation load as function of frequency $\quad[\mathrm{N}]$

influence of importance of structural element

aerodynamic lift load $[\mathrm{N}]$

material effect [-]

highest sample frequency of interest $[\mathrm{Hz}]$

lowest sample frequency of interest $\quad[\mathrm{Hz}]$

total hydrodynamic load per unit length $\quad[\mathrm{N} / \mathrm{m}]$

normalising factor for JONSWAP spectrum

first natural frequency $\quad[\mathrm{Hz}]$

Nyquist frequency $\quad[\mathrm{Hz}]$

peak frequency of the spectrum: $1 / T_{p} \quad[\mathrm{~Hz}]$

frequency of the $q^{\text {th }}$ Fourier component $[\mathrm{Hz}]$

effect of mean stress

ship-impact load $[\mathrm{N}]$

thickness effect

tower top load [N]

effect of weld shape

wave frequency $[\mathrm{Hz}]$

load in $x$-direction $\quad[\mathrm{N}]$

mean zero-crossing frequency: $1 / T_{z} \quad[\mathrm{~Hz}]$

acceleration due to gravity $\left[\mathrm{m} / \mathrm{s}^{2}\right]$

gust factor for a gust of duration $(t)$

incident wave height [m]

breaking wave height $[H / d=0.78] \quad[\mathrm{m}]$

maximum wave height [m]

half power bandwidth $\quad[\mathrm{Hz}]$

significant wave height [m]

significant wave height for return period of 50 years $\quad$ [m] 


\begin{tabular}{|c|c|c|}
\hline$H_{v}$ & visually estimated wave height & {$[\mathrm{m}]$} \\
\hline$I_{t}$ & turbulence intensity & {$[\%]$} \\
\hline$k$ & spring stiffness & {$[\mathrm{N} / \mathrm{m}]$} \\
\hline$k_{s}$ & initial modulus of horizontal subgrade reaction & {$[\mathrm{MPa} / \mathrm{m}]$} \\
\hline$k_{\text {tower }}$ & tower spring stiffness & {$[\mathrm{N} / \mathrm{m}]$} \\
\hline$k_{w}$ & Weibull shape parameter & {$[-]$} \\
\hline$k_{\text {wave }}$ & wave number $=2 \pi / \lambda_{\text {wave }}$ & {$\left[\mathrm{m}^{-1}\right]$} \\
\hline$k_{x x}$ & parameter for soil spring matrix & {$[\mathrm{N} / \mathrm{m}]$} \\
\hline$k_{x \phi}$ & parameter for soil spring matrix & {$[\mathrm{Nm} / \mathrm{m}]$} \\
\hline$k_{\phi x}$ & parameter for soil spring matrix & {$[\mathrm{N} / \mathrm{deg}]$} \\
\hline$k_{\phi \phi}$ & parameter for soil spring matrix & {$[\mathrm{Nm} / \mathrm{deg}]$} \\
\hline$L$ & tower height & {$[\mathrm{m}]$} \\
\hline LAT & lowest astronomical tide, reference level & {$[\mathrm{m}]$} \\
\hline$L_{v}$ & integral length scale of wind model & [m] \\
\hline$m$ & mass & {$[\mathrm{kg}]$} \\
\hline$M$ & moment & {$[\mathrm{Nm}]$} \\
\hline$\hat{M}_{i}$ & amplitude of hydrodynamic inertia moment & {$[\mathrm{Nm}]$} \\
\hline$\hat{M}_{d}$ & amplitude of hydrodynamic drag moment & {$[\mathrm{Nm}]$} \\
\hline$m_{0}$ & spectral moment of order 0 & {$[\ldots]$} \\
\hline$m_{l}$ & spectral moment of order 1 & {$[\ldots]$} \\
\hline$m_{2}$ & spectral moment of order 2 & {$[\ldots]$} \\
\hline$m_{4}$ & spectral moment of order 4 & {$[\ldots]$} \\
\hline$m_{n}$ & spectral moment of order $n$ & {$[\ldots]$} \\
\hline$m_{\text {rep }}$ & representative mass for a 1 DOF system & {$[\mathrm{kg}]$} \\
\hline$m_{s i}$ & displacement mass of impacting vessel & {$[\mathrm{kg}]$} \\
\hline$m_{S N}$ & inverse slope of $S$ - $N$ curve & {$[-]$} \\
\hline MSL & mean sea level & {$[\mathrm{m}]$} \\
\hline$m_{t o p}$ & top mass & {$[\mathrm{kg}]$} \\
\hline$m_{\text {tower }}$ & tower mass & {$[\mathrm{kg}]$} \\
\hline$n$ & counter $1,2, \ldots N$ & {$[-]$} \\
\hline$N$ & even number of equally spaced time steps $\Delta t$ & {$[-]$} \\
\hline$N_{b}$ & number of blades & {$[-]$} \\
\hline$N_{F F T}$ & number of sample records in FFT procedure & {$[-]$} \\
\hline$n_{i}$ & number of stress range variations $S_{i}$ & {$[-]$} \\
\hline$N_{i}$ & allowable number of variations for stress range $S_{i}$ & {$[-]$} \\
\hline$N N$ & power of FFT length: $2^{N N}$ & {$[-]$} \\
\hline$P$ & turbine power & {$[\mathrm{W}]$} \\
\hline$P\left(s_{i}\right)$ & cumulative probability distribution of stress peaks & {$[-]$} \\
\hline$P\left(S_{i}\right)$ & cumulative probability distribution of stress ranges & {$[-]$} \\
\hline$P_{0, s i}$ & minimum crushing strength of impacting vessel part & {$[\mathrm{N}]$} \\
\hline$P_{\text {Dirlik }}$ & Dirlik cumulative probability distribution & {$[-]$} \\
\hline$P_{N}$ & cumulative probability of the standard normal distribution & {$[-]$} \\
\hline$P_{\text {Rayleigh }}$ & Rayleigh probability distribution & {$[-]$} \\
\hline
\end{tabular}




\begin{tabular}{|c|c|c|}
\hline$P_{\text {Rice }}$ & Rice probability distribution & {$[-]$} \\
\hline$q$ & counter $1,2, \ldots$ end & {$[-]$} \\
\hline$Q_{D}$ & Dirlik parameter & {$[-]$} \\
\hline$r$ & distance of blade element to axis of rotation & {$[\mathrm{m}]$} \\
\hline$R$ & radius $(1 / 2 D)$ & {$[\mathrm{m}]$} \\
\hline$R_{D}$ & Dirlik parameter & {$[-]$} \\
\hline$R_{\text {root }}$ & radius of the blade root & {$[\mathrm{m}]$} \\
\hline$R_{\text {tip }}$ & radius of the blade tip & [m] \\
\hline$S(f)$ & single sided density spectrum & {$[\ldots]$} \\
\hline$s(t)$ & time varying stress & {$\left[\mathrm{N} / \mathrm{m}^{2}\right]$} \\
\hline$S_{F}(f)$ & load spectrum & {$\left[\mathrm{N}^{2} \mathrm{~s}\right]$} \\
\hline$S_{\text {Ftop }}(f)$ & tower top load spectrum & {$\left[\mathrm{N}^{2} \mathrm{~s}\right]$} \\
\hline$S_{i}$ & $i^{t h}$ stress range class of $s(t)$ & {$\left[\mathrm{N} / \mathrm{m}^{2}\right]$} \\
\hline$s_{i}$ & $i^{t h}$ peak of $s(t)$ & {$\left[\mathrm{N} / \mathrm{m}^{2}\right]$} \\
\hline$S_{J S}(f)$ & JONSWAP (wave) spectrum & {$\left[\mathrm{m}^{2} \mathrm{~s}\right]$} \\
\hline$S_{\text {Kaimal }}(f)$ & Kaimal (turbulence) spectrum & {$\left[(\mathrm{m} / \mathrm{s})^{2} \mathrm{~s}\right]$} \\
\hline$S_{\text {Kármán }}(f)$ & Von Kármán (turbulence) spectrum & {$\left[(\mathrm{m} / \mathrm{s})^{2} \mathrm{~s}\right]$} \\
\hline$S_{P M}(f)$ & Pierson-Moskowitz (wave) spectrum & {$\left[\mathrm{m}^{2} \mathrm{~s}\right]$} \\
\hline$S_{u}$ & displacement spectrum & {$\left[\mathrm{m}^{2} \mathrm{~s}\right]$} \\
\hline$s_{u}$ & undrained shear stress & {$\left[\mathrm{N} / \mathrm{m}^{2}\right]$} \\
\hline$S_{v}(f)$ & turbulence spectrum & {$\left[(\mathrm{m} / \mathrm{s})^{2} \mathrm{~s}\right]$} \\
\hline$t$ & time & {$[\mathrm{s}]$} \\
\hline$T$ & duration of measurement $T=N \Delta t$ & {$[\mathrm{~s}]$} \\
\hline$T_{c}$ & average crest period & [s] \\
\hline$T_{d}$ & duration of environmental state & {$[\mathrm{s}]$} \\
\hline$T_{i}$ & incident wave period & {$[\mathrm{s}]$} \\
\hline$T_{m}$ & mean period & [s] \\
\hline$T_{p}$ & peak period (of peak in spectrum) & [s] \\
\hline$T_{r}$ & time length of sub-record & {$[\mathrm{s}]$} \\
\hline$T R F$ & transfer function & {$[\ldots]$} \\
\hline$T_{r-F F T}$ & length of sub-record for FFT of time series & {$[\mathrm{s}]$} \\
\hline$T_{s}$ & average period between stress ranges & {$[\mathrm{s}]$} \\
\hline$T_{\text {total }}$ & total time length of record & {$[\mathrm{s}]$} \\
\hline$t_{w}$ & tower wall thickness & {$[\mathrm{m}]$} \\
\hline$T_{z}$ & mean zero-crossing period & [s] \\
\hline$u$ & horizontal water particle velocity due to wave motion & {$[\mathrm{m} / \mathrm{s}]$} \\
\hline$\dot{u}$ & horizontal water particle acceleration due to wave motion & {$\left[\mathrm{m} / \mathrm{s}^{2}\right]$} \\
\hline$U_{c}(z)$ & current velocity at elevation $z$ & {$[\mathrm{~m} / \mathrm{s}]$} \\
\hline$U_{c 0}$ & current velocity at the sea surface $z=0$ & {$[\mathrm{~m} / \mathrm{s}]$} \\
\hline$U_{c 0, \max }$ & maximum current velocity at the sea surface & {$[\mathrm{m} / \mathrm{s}]$} \\
\hline$u_{\text {top }}$ & tower top displacement & {$[\mathrm{m}]$} \\
\hline$V_{0}$ & undisturbed wind velocity & {$[\mathrm{m} / \mathrm{s}]$} \\
\hline$V_{\text {cut-in }}$ & turbine cut-in wind speed & {$[\mathrm{m} / \mathrm{s}]$} \\
\hline$V_{\text {cut-out }}$ & turbine cut-out wind speed & {$[\mathrm{m} / \mathrm{s}]$} \\
\hline
\end{tabular}




\begin{tabular}{|c|c|c|}
\hline$V_{d i r}$ & wind direction & [deg] \\
\hline$V_{\text {disk }}$ & wind velocity at the actuator disk & {$[\mathrm{m} / \mathrm{s}]$} \\
\hline$V_{\text {gust }}$ & wind speed in gust & {$[\mathrm{m} / \mathrm{s}]$} \\
\hline$V_{\text {rated }}$ & rated wind speed of turbine & {$[\mathrm{m} / \mathrm{s}]$} \\
\hline$V_{\text {rel }}$ & relative wind speed at blade element & {$[\mathrm{m} / \mathrm{s}]$} \\
\hline$V_{\text {rot }}$ & rotation speed at blade element & {$[\mathrm{m} / \mathrm{s}]$} \\
\hline$V_{\text {section }}$ & relative wind speed at blade section & {$[\mathrm{m} / \mathrm{s}]$} \\
\hline$v_{s i}$ & velocity of impacting vessel & {$[\mathrm{m} / \mathrm{s}]$} \\
\hline$V_{\text {tip }}$ & speed of blade tip & {$[\mathrm{m} / \mathrm{s}]$} \\
\hline$V_{w}$ & mean wind speed & {$[\mathrm{m} / \mathrm{s}]$} \\
\hline$V_{w}(z)$ & mean wind speed at height $z$ & {$[\mathrm{~m} / \mathrm{s}]$} \\
\hline$V_{w, \text { ext } 10 \mathrm{~min}}$ & extreme 10 minute mean wind speed & {$[\mathrm{m} / \mathrm{s}]$} \\
\hline$V_{w, \text { ext } 1 h}$ & extreme 1 hour mean wind speed & {$[\mathrm{m} / \mathrm{s}]$} \\
\hline$V_{w, r}$ & mean wind speed at the reference height $z_{r}$ & {$[\mathrm{~m} / \mathrm{s}]$} \\
\hline$V_{w, \text { year }}$ & yearly mean wind speed & {$[\mathrm{m} / \mathrm{s}]$} \\
\hline$x$ & displacement in $x$-direction & {$[\mathrm{m}]$} \\
\hline$\dot{x}$ & velocity in $x$-direction & {$[\mathrm{m} / \mathrm{s}]$} \\
\hline$\ddot{x}$ & acceleration in $x$-direction & {$\left[\mathrm{m} / \mathrm{s}^{2}\right]$} \\
\hline$\hat{x}$ & amplitude of displacement & {$[\mathrm{m}]$} \\
\hline$\hat{x}(f)$ & amplitude of displacement as function of frequency & [m] \\
\hline$x(t)$ & displacement as function of time in $x$-direction & {$[\mathrm{m}]$} \\
\hline$x_{0}$ & initial displacement in $x$-direction & {$[\mathrm{m}]$} \\
\hline$x_{m}$ & Dirlik parameter & {$[-]$} \\
\hline$x x$ & arbitrary number & {$[-]$} \\
\hline$y$ & horizontal displacement in soil & {$[\mathrm{m}]$} \\
\hline$z$ & $\begin{array}{l}\text { vertical co-ordinate, measured positively upwards from } \\
\text { mean sea level }\end{array}$ & {$[\mathrm{m}]$} \\
\hline$z(t)$ & vertical displacement as function of time & [m] \\
\hline$z_{0}$ & surface roughness length & {$[\mathrm{m}]$} \\
\hline$z_{\text {combinedwave }}(t)$ & water surface elevation at time $t$ & {$[\mathrm{~m}]$} \\
\hline$Z_{D}$ & Dirlik parameter & {$[-]$} \\
\hline$z_{n}$ & discrete vertical displacement at step $n$ & [m] \\
\hline$z_{r}$ & reference height & {$[\mathrm{m}]$} \\
\hline$z_{\text {wave }}(t)$ & water surface elevation at time $t$ & {$[\mathrm{~m}]$} \\
\hline$\Delta f$ & frequency step & {$[\mathrm{Hz}]$} \\
\hline$\Delta r$ & radial length of blade element & {$[\mathrm{m}]$} \\
\hline$\Delta t$ & time step & {$[\mathrm{s}]$} \\
\hline$\Delta z$ & height of impact region & {$[\mathrm{m}]$} \\
\hline$\Delta \sigma_{R}$ & reference fatigue strength at $N_{i}=5 \cdot 10^{6}$ & {$\left[\mathrm{~N} / \mathrm{m}^{2}\right]$} \\
\hline$\Omega_{R}$ & rotor angular velocity & {$[\mathrm{rad} / \mathrm{s}]$} \\
\hline$\alpha$ & angle of attack & {$[\mathrm{deg}]$} \\
\hline$\alpha_{\text {cur }}$ & hydrodynamic current exponent, usually $1 / 7$ & {$[-]$} \\
\hline$\alpha_{\text {shear }}$ & power law coefficient for wind shear & {$[-]$} \\
\hline
\end{tabular}




$\begin{array}{ll}\beta & \text { fraction of critical damping coefficient } \\ \hat{\zeta} & \text { wave amplitude } \\ \varepsilon & \text { spectral width parameter } \\ \varepsilon_{S} & \text { material strain } \\ \varepsilon_{y} & \text { yield strain } \\ \varepsilon_{50} & \text { strain at } 50 \% \text { of the maximum stress } \\ \phi & \text { angle of inflow } \\ \phi^{\prime} & \text { friction angle of sand } \\ \gamma_{D} & \text { Dirlik parameter } \\ \gamma_{J S} & \text { peak enhancement factor for JONSWAP spectrum } \\ \varphi & \text { phase angle } \\ \varphi_{\text {tower }} & \text { angle of tower rotation } \\ \varphi_{\text {wave }} & \text { wave phase angle } \\ \lambda & \text { tip speed ratio } \\ \lambda_{\text {wave }} & \text { wavelength } \\ \mu & \text { tower mass per meter } \\ \theta & \text { pitch angle } \\ \rho_{\text {air }} & \text { density of air } \\ \rho_{\text {soil }} & \text { density of soil } \\ \rho_{\text {steel }} & \text { density of steel } \\ \rho_{\text {water }} & \text { water density } \\ \sigma & \text { standard deviation } \\ \sigma_{a} & \text { JONSWAP slope coefficient for left-hand side } \\ \sigma_{b} & \text { JONSWAP slope coefficient for right-hand side } \\ \sigma_{J S} & \text { JONSWAP slope coefficient } \\ \sigma_{s} & \text { stress } \\ \sigma_{v} & \text { standard deviation of the wind speed } \\ \sigma_{y} & \text { yield stress } \\ & \end{array}$

[-]

[m]

[-]

[-]

[-]

[\%]

[deg]

[deg]

[-]

[-]

[rad/s]

[deg]

[rad]

[-]

[m]

[kg/m]

[deg]

$\left[\mathrm{kg} / \mathrm{m}^{3}\right]$

$\left[\mathrm{kg} / \mathrm{m}^{3}\right]$

$\left[\mathrm{kg} / \mathrm{m}^{3}\right]$

$\left[\mathrm{kg} / \mathrm{m}^{3}\right]$

[...]

[-]

[-]

[-]

$\left[\mathrm{N} / \mathrm{m}^{2}\right]$

$[\mathrm{m} / \mathrm{s}]$

$\left[\mathrm{N} / \mathrm{m}^{2}\right]$ 


\section{Introduction}

\subsection{Offshore wind}

Wind has been a source of energy for mankind for thousands of years. Only the industrial revolution marked a temporary end in its dominance of the energy supply. An effort by large companies in the 1970s to re-introduce wind as a powerful source of energy all but failed, to be overtaken and surpassed by environmentally-conscious do-ityourself projects by individuals and small companies. Their slow but firm continuation evolved to the multi-billion-euro business that it is today [1].

Wind energy onshore nowadays is a mature industry responsible for meeting a part of the energy needs in countries around the world. Though care for the environment is a large driver as far as the public opinion and political opportunism are concerned, for the active players in this field it is just business. Part of this business is to find the best locations to harvest the energy from the wind. With the best onshore spots already occupied and neighbours complaining aplenty in an overcrowded Europe, it was a natural step to take wind offshore. Moreover, the wind resources are even more abundant and of better quality at sea

Like many advances in technology, offshore wind received a nudge in the right direction from national and European development programmes. Subsequent to a number of large research projects, offshore demonstration wind turbines were erected in Sweden, Denmark and The Netherlands in the early 1990s. Figure 1.1 shows the Nogersund turbine in Sweden (1990), Vindeby in Denmark (1991) and Lely in The Netherlands (1995).
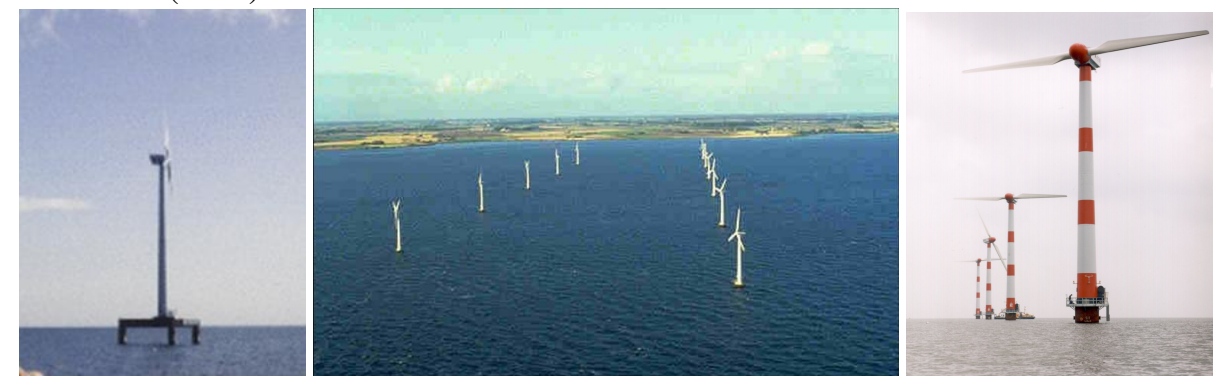

Figure 1.1 Demonstration offshore wind projects: Nogersund (S), Vindeby (DK) and Lely (NL)

At the end of the 1990s, wind turbines had evolved to multi-megawatt machines. At Blyth, UK, two Vestas V66 2MW turbines were installed offshore to demonstrate the applicability in the harsher environment of the North Sea. This project was followed not long after by several commercial projects in Scandinavian waters to culminate in the first large scale offshore wind power station of 80 turbines at Horns Rev in 2002. 
Promoted by suitable support mechanisms, Denmark, Ireland, the UK and Germany are progressing in offshore wind turbine installation. The effect of these efforts is that practical experience is building up resulting in better understandin of the risks involved which will eventually make offshore wind cost effective even without support mechanisms. Already $500 \mathrm{MW}$ has been installed and plans for another 27,000 MW are on the drawing boards up to 2009 [2]. Figure 1.2 gives an overview of the installed and planned offshore wind farms.

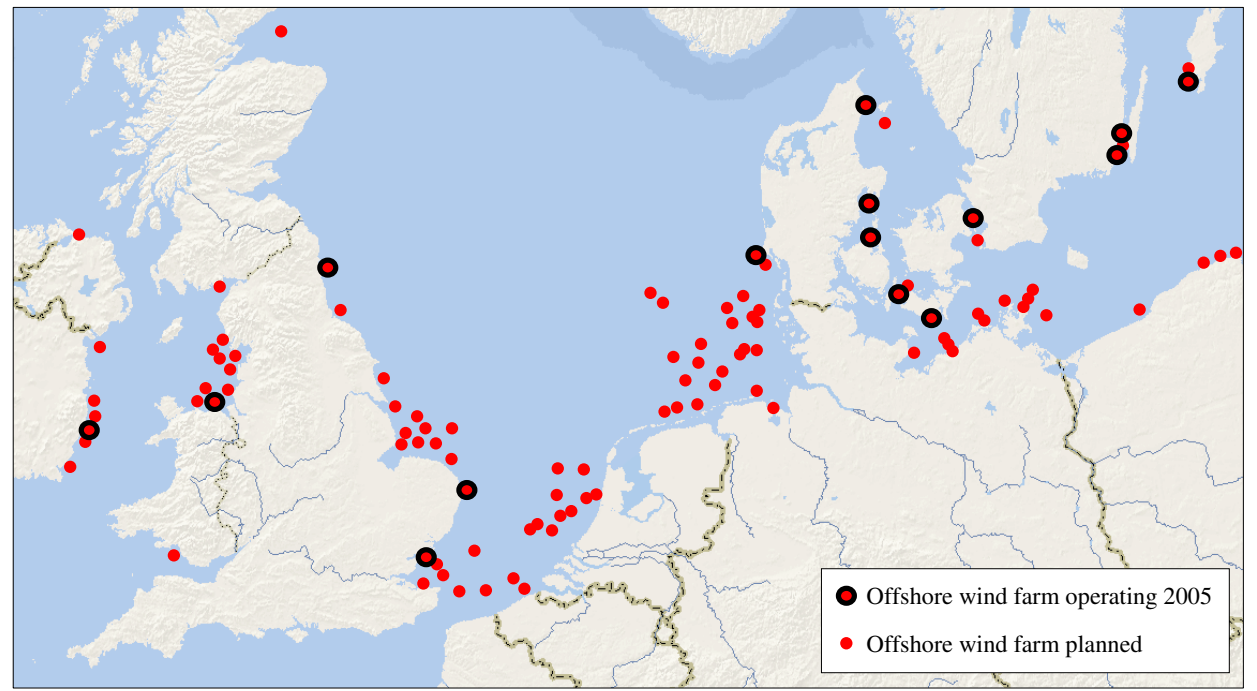

Figure 1.2 Overview of existing and planned offshore wind farms in Europe

\subsection{Objective of this study}

To improve the cost-effectiveness of offshore wind and make it a mainstream source of energy, the design drivers should be known in depth to reduce risk and optimise structures.

Offshore engineering and wind energy engineering are both mature fields of technology. The combination of these fields to create offshore wind turbines is not always just a simple summation. The objective of the study reported in this thesis is to explore the basics of the parent technologies and, with measurements and simulations, the effects of the combination. The final goal is to: "Devise a basis for design of Offshore Wind Turbine Support Structures".

The most common type of support structure used so far is the monopile. This type of structure is therefore the main focus point of this study. The general expectation is that with larger, heavier turbines in deeper water, the tripod and other types of structures will 
be good candidates for future offshore wind turbines. The design approach is tailored for monopiles but can in the future also be applied to alternative structures.

\subsection{Study approach}

\subsubsection{Four reference sites}

To enhance the practicality of this academic thesis, four actual offshore wind farms have been selected to serve as reference. The four sites have their own specific characteristics making this thesis applicable to generic offshore wind turbine design. The sites are briefly described below; their locations are shown in Figure 1.3.

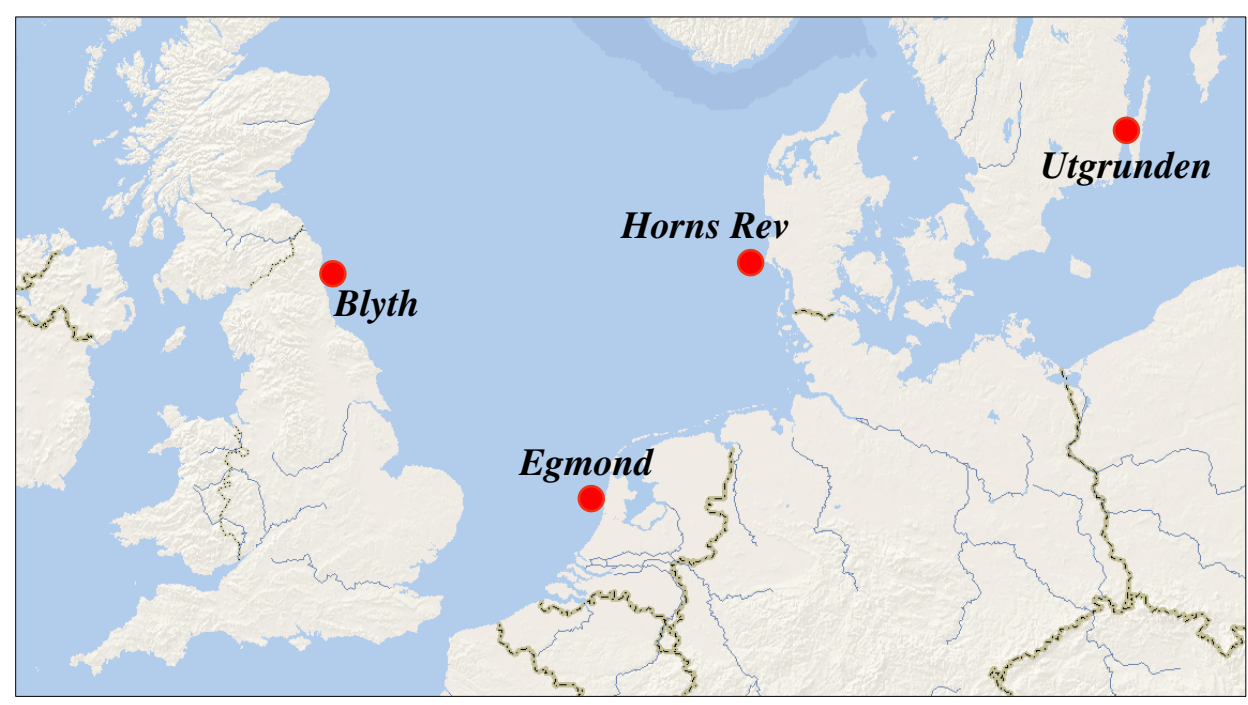

Figure 1.3 North and Baltic Sea with 4 locations

Blyth

At Blyth two Vestas V66 2 MW turbines are in operation since 1999. The turbine installation was sponsored by the European Union, as was the research project, which was focussed on scientific monitoring of these turbines. This project was named OWTES for "Design Methods for Offshore Wind Turbines at Exposed Sites" [3]. The project was finished in the summer of 2003 and delivered a large number of very relevant reports and a database with load and response measurements.

The turbines are installed on a rocky outcrop called the North Spit. The most specific characteristics of this site are the rocky seabed and the distorted wave pattern due to the Spit. Furthermore, the site is located along the East coast of the UK in an area where the strongest winds come from the west over land which reduces the wave action compared to other, more exposed sites. 


\section{Horns Rev}

The 80 turbines off the West coast of Denmark are a typical example of future large offshore wind farms. Extensive pre-design information and soil, wind and wave data are available. The location is completely exposed to the governing westerly winds and waves and is exposed to high tidal currents. The offshore wind farm produces a maximum of $160 \mathrm{MW}$, which is enough to cover $2 \%$ the electricity consumption of Denmark. The wind farm has been operating since the end of 2002.

\section{Utgrunden}

This Swedish offshore wind farm was also a subject of study by Kühn [4]. It consists of 7 Tacke (now GE) 1.5 MW turbines, which are built on a sand bank in the Kalmarsund between the Swedish mainland and Öland. The wind farm was commissioned in 2000. Wave loads are not so extreme as at the North Sea locations, but the Baltic Sea may be covered with ice in the winter.

\section{OWEZ}

During this study project the Dutch demonstration offshore wind farm was designed and will be constructed while this thesis is being printed. The offshore wind farm will be located $10 \mathrm{~km}$ off the coast of Egmond aan Zee and has been named Offshore Wind farm Egmond aan Zee, OWEZ in short. It will comprise 36 Vestas V90 turbines, giving it a maximum output of $108 \mathrm{MW}$. Participation in the design process for this offshore wind farm gave the opportunity to test the theoretical approach directly in a real project. The site had already been investigated during the Opti-OWECS study [5].

\subsubsection{Validation, variation and application}

With the installation of actual offshore wind turbines, it finally became possible to perform measurements and validate the various models created in desktop studies. A major part of this report is therefore based on this model validation. Especially the extensive measurements in the OWTES project are of great value in this respect [3].

When the specific models and modelling in general are found to agree with reality, the models are used for parameter variation. It is expected that support structure design is very site dependent. By parameter variation, mainly based on the reference sites mentioned in the previous section, the site dependency of various aspects can be tested and recommendations for a generic design approach can be drafted.

These recommendations can be used in two phases of the design process. First, a-priori knowledge of general behaviour of offshore wind turbines can assist the designer in in the front-end engineering phase giving reliable preliminary estimates of critical design aspects. Second, detailed design of various sub-components can be performed fully or partially uncoupled from the offshore wind turbine. 


\subsection{Thesis outline}

The thesis gives a number of case descriptions, based on the reference sites described in section 1.3.1. Chapter 2 provides the general background: offshore and wind energy terminology is introduced and where needed adapted to fit the specifics of offshore wind turbines. Because the design of offshore wind turbines leans heavily on time varying, stochastic processes, the basics of stochastics are summarized. The general calculation methods for wave, current and wind loads are reviewed with a focus on their application to offshore wind turbines. Finally, general turbine characteristics are summarized and the calculation methods of turbine loads are presented to introduce the overall offshore wind turbine dynamics.

The general design methodology for offshore wind turbines is illustrated in chapter 3 following available design documents of the four reference sites. The methodology is compared to a design of an offshore jacket for the oil and gas industry. The design is presented in three distinct parts: extreme load checks, fatigue damage checks and design details. Of these areas, for fatigue damage assessment no consensus yet exists for offshore wind turbine design. This will therefore be the focal point of the subsequent chapters.

Chapter 4 gives an overview of frequency domain calculations in general and the analysis of fatigue due to wave loading in particular. Methods to apply the frequency domain approach on wind turbine response calculations are explored in chapter 5 and applied to a model of the Blyth turbine in chapter 6 . The method is also checked against measurements at the Blyth turbine. A practical frequency domain method has been developed and in chapter 7 the method is applied to the design of the OWEZ design. As the method enables parameter variation to be performed very easily, the effects of different aerodynamic damping ratio's, wave parameters and scour depths on the support structure dynamics and fatigue are calculated. Chapter 8 summarizes the conclusions and gives an outlook on the further development of offshore wind turbine design practice.

\subsection{Software used}

The following computer programs were used in this thesis:

- BLADED, a commercial (offshore) wind turbine design package, Garrad Hassan \& Partners Ltd.

- SESAM, offshore structural design package, Det Norske Veritas

- ANSYS, general purpose finite element program, Anys Inc.

- MATLAB, general purpose mathematical modelling program, MathWorks Inc.

- RECAL, Matlab tool for offshore wind turbine modelling, DUT

- EXCEL, spreadsheet program, Microsoft Inc. 


\section{Basics of offshore, wind and turbines}

\subsection{Introduction}

The technology of offshore wind energy is based on two independent and self-relying engineering fields. To enhance communication and mutual understanding between these fields a common language should be developed. When creating such a language the aim should always be to make things clearer and easier. Enforcing difficult, though literally perhaps correct, terms on "the other party" will only enlarge the communications gap. From a scientific point of view an effort to make things easier may contradict a purely scientific approach of defining entities completely and correctly. A good example of terminology difficulties is the following.

\footnotetext{
According to people in the wind-business, the term windmill should not be used for wind turbines: nothing is being milled or ground. When defining an offshore wind turbine, the entire system could be named an Offshore Wind Energy Converter (OWEC). But this full description is too long and OWEC sounds strange. People will rather use the more intuitive Offshore Wind Turbine in speaking and the abbreviation OWT in writing. But how then to define the parts on top of the support structure? Using the term "turbine" would not be confusing when OWEC was used, but "offshore wind turbine" and "turbine" together can easily lead to confusion. Then, maybe, the best term to use for the entire system would be Offshore Windmill, always in full, and turbine for nacelle and rotor. However, Offshore Windmill is not commonly accepted.

In this thesis the entire system will always be referred to as "offshore wind turbine", while the rotor-nacelle assembly will always be referred to as "RNA". according to the IEC standard [65].
}

Section 2.2 reviews general offshore and wind energy terminology and defines new terms specific for offshore wind energy where necessary. As wind and waves are time varying stochastic processes, the basics of stochastics are reviewed in section 2.3. The next two sections describe wave and wind conditions and the calculation methods of wave, current and wind loads on structures. Section 2.6 gives an overview of all specific features of modern day turbines that can have an impact on the design of the support structure. The calculation methods for loads on and in the turbine are explained. Section 2.7 then combines turbine and wind and wave dynamics. The basics of dynamics are reviewed in the light of offshore wind turbine design. Section 2.8 gives an overview of 
the fatigue process and how to determine fatigue damage, with particular application to the support structure. The final section 2.9 describes soil and foundations.

\subsection{General terminology}

An overview of the terminology chosen for this thesis is shown in Figure 2.1.

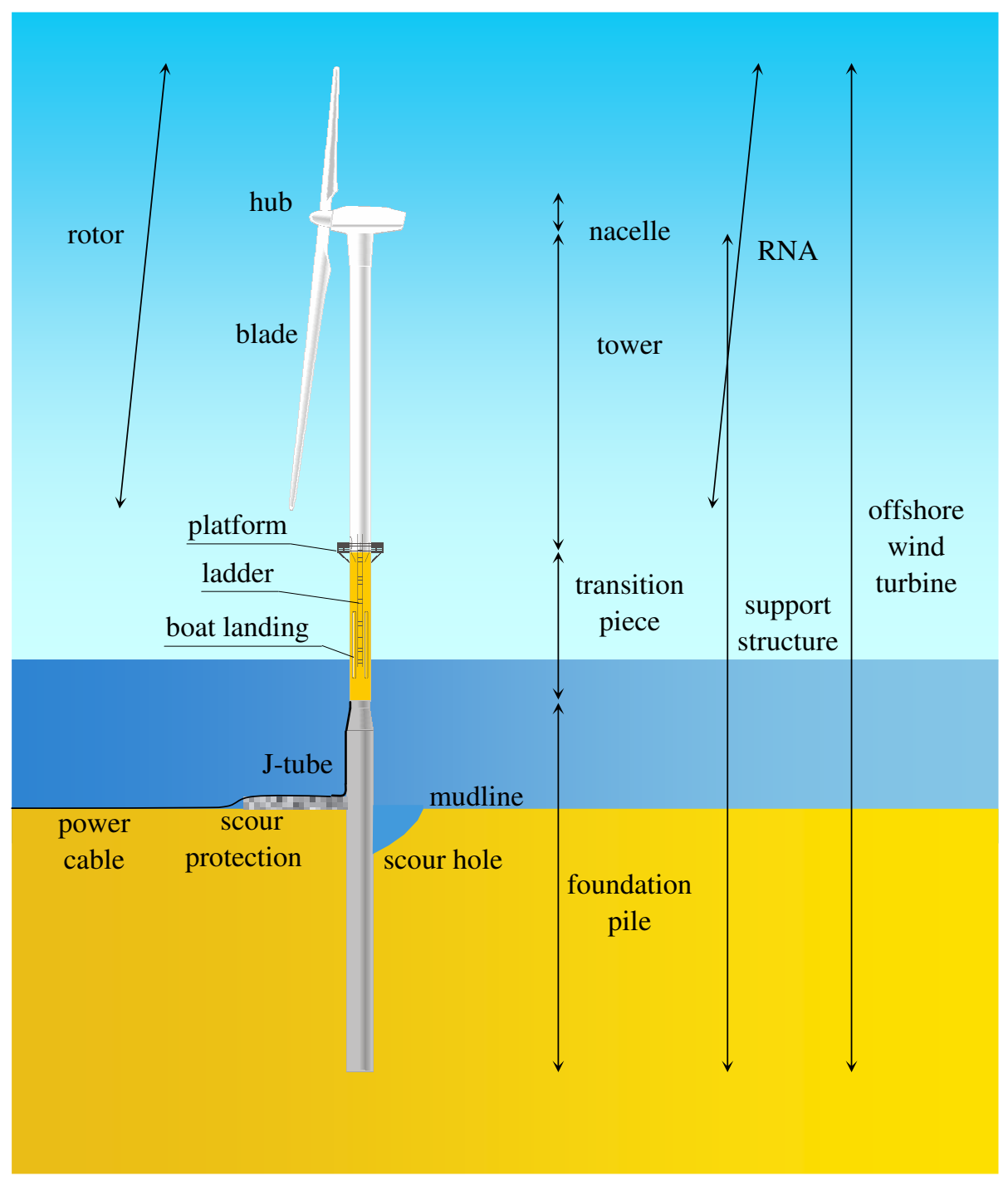

Figure 2.1 Overview of offshore wind turbine terminology 


\subsection{Stochastic or random processes}

\subsubsection{Introduction}

Loads on, and consequently responses of offshore wind turbines vary in time. For analysis of time series in the time domain mainly generalities can be distinguished: maximum, minimum, mean, strange peaks or slow variations. To make the data more accessible, time series can be transformed into the frequency domain. The data can then be plotted as a spectrum. Figure 2.2a shows a measured time series of the mudline bending stress of an offshore wind turbine. Figure $2.2 \mathrm{~b}$ shows the corresponding spectrum created from this data. In the spectrum several characteristic responses can be distinguished far more easily.
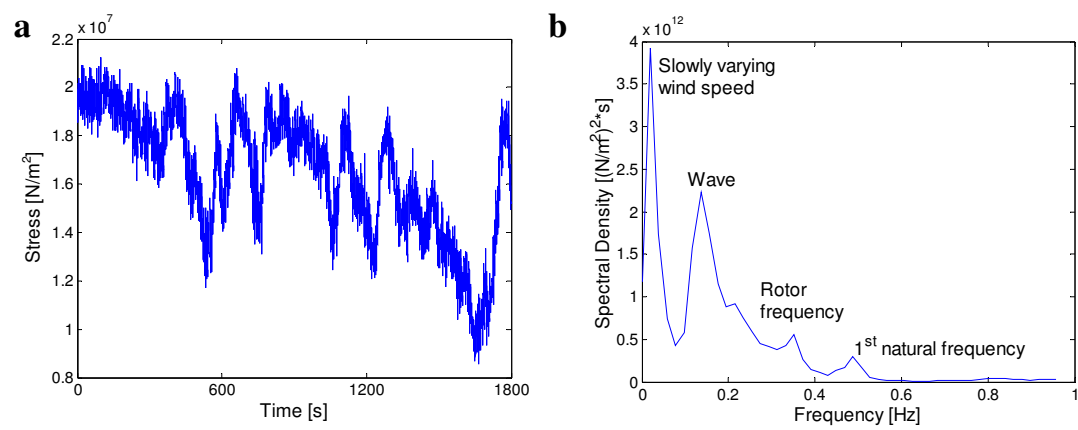

Figure 2.2 (a) Time domain record of measured mudline bending stress variation

(b) The frequency domain spectrum of the same time trace

\subsubsection{Description of random data}

When designing structures, two typical time related phenomena can be distinguished:

1. Deterministic events: the event can be repeated and the behaviour of the event can be predicted reasonably well through knowledge of the physics or through previous measurements. An emergency stop of the turbine or a vessel colliding with an offshore wind turbine are examples of deterministic events.

2. Random or stochastic events: a typical time-varying event that cannot be reproduced or predicted in detail. To understand these processes "one should conceptually think in terms of all time history records that could have occurred" [6]. Examples are wind and wave excitation.

Analysis of random data is enhanced when the statistical parameters of a random process remain constant for every realisation of a certain event. When this is true, the data of the event is called stationary. Sea wave fields also called "sea state", for instance, are generally assumed to be stationary for a reference period of 3 hours. 
To transform a time series to the frequency domain, Fourier transformation is used. The basis of Fourier transformations is the assumption that random signals can be represented by the sum of a number of sinusoids or wavelets, each with a specific amplitude, frequency and phase angle. This can be demonstrated in a simple Excel-sheet example. Three sine waves are defined and their discrete elevation per time step $z(t)$ is calculated for a period of $4 \mathrm{~s}$ at an interval of $0.1 \mathrm{~s}$. The waves are of the form:

$$
z_{\text {wave }}(t)=A_{\text {wave }} \sin \left(f_{\text {wave }} \cdot 2 \pi \cdot t+\varphi_{\text {wave }}\right)
$$

with:

\begin{tabular}{l|l|l}
\hline$z_{\text {wave }}(t)$ & elevation at time $t$ & {$[\mathrm{~m}]$} \\
$A_{\text {wave }}$ & wave amplitude & {$[\mathrm{m}]$} \\
$f_{\text {wave }}$ & wave frequency & {$[\mathrm{Hz}]$} \\
$\varphi_{\text {wave }}$ & wave phase angle & {$[\mathrm{rad}]$} \\
$t$ & time & {$[\mathrm{s}]$}
\end{tabular}

From 3 arbitrary regular waves, a combined wave can be made as shown in Figure 2.3.
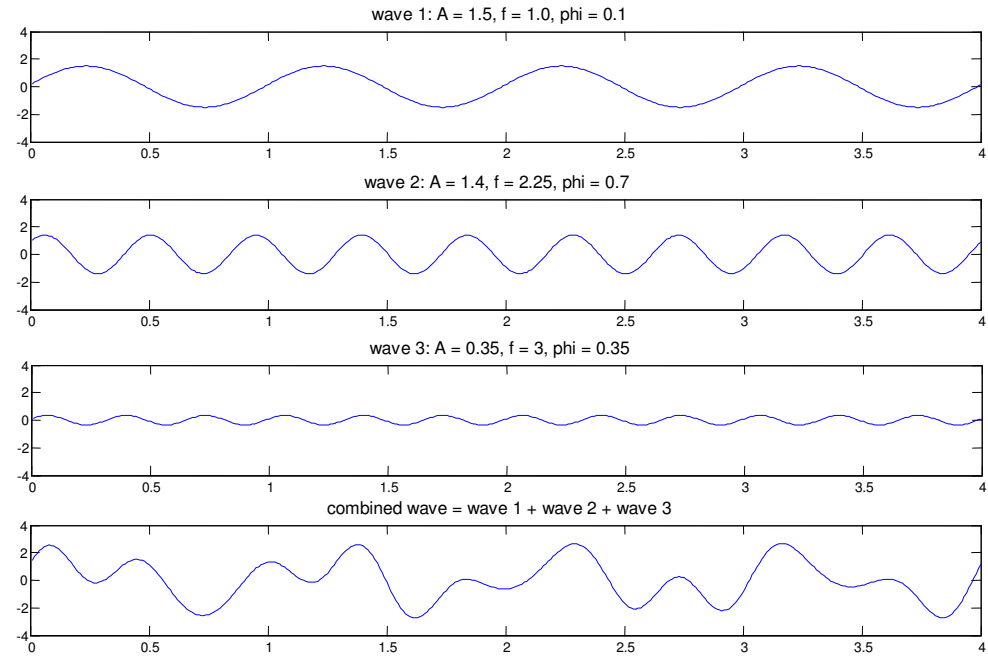

Figure 2.3 Three regular waves combined to an irregular wave

In this case we know the exact mathematical description of the three waves from which the combined wave was constructed and therefore the exact description of the combined wave: 


$$
z_{\text {combinedwave }}(t)=\sum_{\text {wave }=1}^{3} A_{\text {wave }} \sin \left(f_{\text {wave }} \cdot 2 \pi \cdot t+\varphi_{\text {wave }}\right)
$$

\subsubsection{Fourier series representation of random data}

If we now reverse the approach by assuming that the combined wave is available as a measurement and assume that it is composed of a finite number of discrete waves, Fourier transform provides a mathematical tool to reproduce the sine waves making up the combined wave. The time period of the measurement is $T$ and the time step is $\Delta t$. To accomplish this reproduction process, equation (2.2) is transformed from single sines with phase angles to a combination of sines and cosines:

$$
z(t)=A_{0}+\sum_{q=1}^{e n d}\left\{A_{q} \cos \left(2 \pi f_{q} t\right)+B_{q} \sin \left(2 \pi f_{q} t\right)\right\}
$$

with:

\begin{tabular}{l|l|l}
$A_{0}$ & mean of the signal (in this case 0) & {$[\mathrm{m}]$} \\
$A_{q}$ and $B_{q}$ & Fourier coefficients & {$[\mathrm{m}]$} \\
$q$ & counter $1,2, \ldots q_{\mathrm{end}}$ & {$[-]$} \\
$f_{q}$ & frequency of $q^{\text {th }}$ Fourier component & {$[\mathrm{Hz}]$} \\
$t$ & time & {$[\mathrm{s}]$}
\end{tabular}

From the theory of Fourier analysis the coefficients $A_{q}$ and $B_{q}$ are defined by:

$$
A_{q}=\frac{2}{T} \int_{0}^{T} z(t) \cos \left(2 \pi f_{q} t\right) d t \text { and } B_{q}=\frac{2}{T} \int_{0}^{T} z(t) \sin \left(2 \pi f_{q} t\right) d t
$$

where the integral can be made discrete with:

\begin{tabular}{l|l|l}
\hline$T$ & duration of measurement $T=N \Delta t$ & {$[\mathrm{~s}]$} \\
$f_{q}$ & $=q \cdot f_{1}=q \cdot \frac{1}{T}=\frac{q}{N \Delta t}$ & {$[\mathrm{~Hz}]$} \\
$t$ & $n \Delta t$ & {$[\mathrm{~s}]$} \\
$\Delta t$ & time step & {$[\mathrm{s}]$} \\
$n$ & counter $1,2, \ldots . N$ & {$[-]$} \\
$N$ & total number of time steps & {$[-]$}
\end{tabular}

which makes equation (2.5) the discrete representation of equation (2.4): 


$$
A_{q}=\frac{2}{N} \sum_{n=1}^{e n d} z_{n} \cos \frac{2 \pi q n}{N} \text { and } B_{q}=\frac{2}{N} \sum_{n=1}^{e n d} z_{n} \sin \frac{2 \pi q n}{N}
$$

The coefficients in equations (2.2) and (2.3) are interchangeable as follows:

$$
A_{\text {wave }}=\sqrt{A_{q}^{2}+B_{q}^{2}} \quad \text { and } \quad \tan \left(\varphi_{\text {wave }}\right)=\frac{B_{q}}{A_{q}}
$$

By applying the Fourier transform to the signal created by adding the three harmonic sine waves in Figure 2.3 a plot can be made of the amplitude $A_{\text {wave }}$ and $\varphi_{\text {wave }}$ as a function of frequency as shown in Figure 2.4. For the amplitude, all values except at the frequencies 1, 2.25 and 3 are zero; for the phase angle, the intermediate frequencies return a random scatter of angles as shown in the right-hand side graph. The values at $f$ $=1,2.25$ and 3 are the exact input of the initial sines.
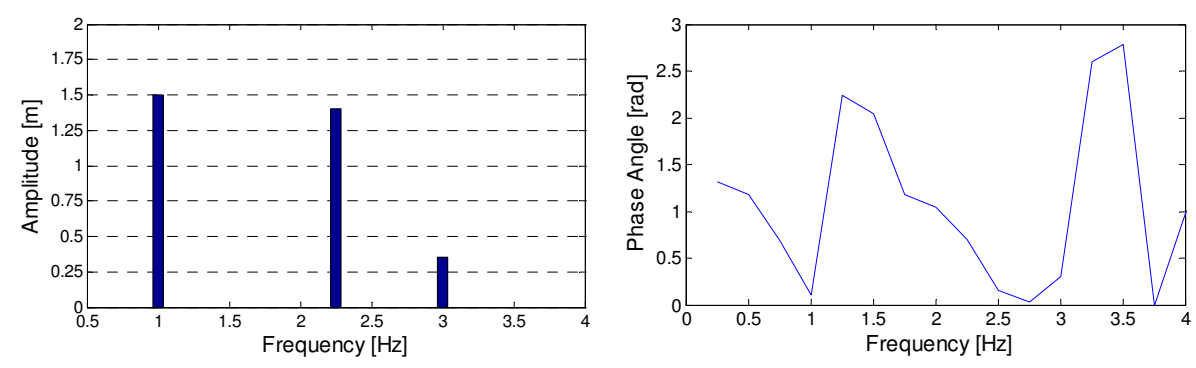

Figure 2.4 Amplitude and phase angle results of Fourier transform per frequency

If all information from the Fourier transform is preserved and re-used to create a new sum of harmonic waves, the exact same random wave signal will be found again.

\subsubsection{Time and frequency domain descriptions}

Although the Fourier transformation can be performed manually, as shown in the previous section, its use is widespread and several algorithms exist that are programmed to perform the transformation in a standard manner on any given signal. The most commonly used algorithm is the Fast Fourier Transform or FFT. The most common output is usually not the amplitude and phase angle as a function of frequency, but the power spectral density per frequency, defined as: $\frac{1}{2}\left(A_{\text {wave }}^{2} / \Delta f\right)=\frac{1}{2}\left\{\left(A_{q}^{2}+B_{q}^{2}\right) T\right\}$ as function of frequency. When the power spectral density is plotted as a function of frequency, we find a power (or energy) density spectrum, psd, auto spectrum or just spectrum. 
A spectrum can also be used to recreate a time signal. By assuming that the phase angle is distributed randomly, harmonic waves can be created based on the power spectral density at each separate frequency, combined with a randomly picked phase angle. The time series created in this way is never the exact copy of the time series the spectrum was retrieved from, but the spectral parameters are the same, provided that the signal is long enough. Figure 2.5 shows the inverse conversion from frequency to time domain as well as the normal transformation from time to frequency domain. For both transformations standard algorithms are available, the most commonly used is the Fast Fourier Transform (FFT) and its Inverse (IFFT).

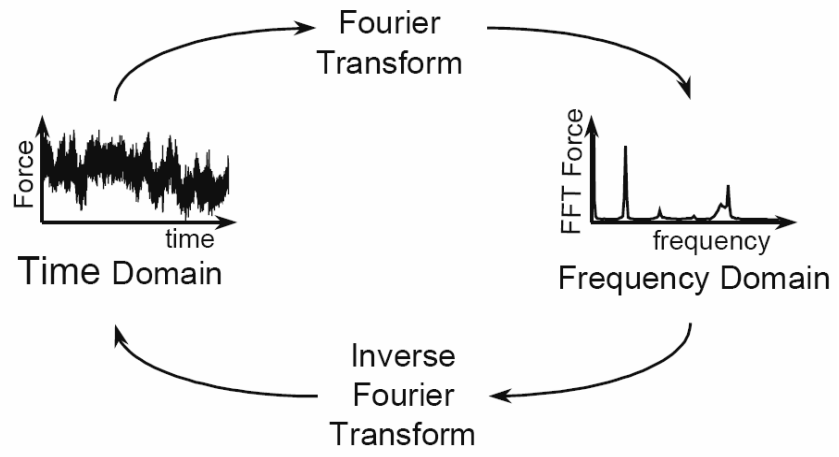

Figure 2.5 Conversion from time domain to frequency domain via FFT and from frequency domain to time domain via IFFT

\subsubsection{Quality control}

\section{Main parameters in the time and frequency domain}

The basis of Fourier transform is the concept that a time varying signal can be described as a sum of sine functions with specific amplitudes, frequencies and phases. The transform provides a calculation method to derive the amplitudes and frequencies from, in this specific case, discrete and finite time records. Let an arbitrary record of $x(t)$ of total length $T$ be sampled at a time step $\Delta t=T / N$, with $N$ being even. This creates a set of $N$ data points $x_{n}$ in the time domain, $n=1,2, \ldots, N$. The representation of $x(t)$ in the frequency domain consists of the sum of sinusoids with frequencies $q \Delta f=q(1 / T)$. The lowest or basic frequency is $f_{\min }=f_{1}=1 / T$, while the highest frequency is obtained for $q=N / 2$, hence $f_{\max }=f_{N / 2}=\mathrm{N} /(2 T)$; see the section 2.3.6.

For FFT a further requirement is that $N$ is a power of 2: 512, 1024, 2048, etc.

\section{Aliasing and the Nyquist frequency}

Fourier analysis of a discretely sampled signal (e.g. a wave record) can erroneously introduce a wave component with a higher frequency, an alias of the original wave component. This effect can be demonstrated as follows. A harmonic wave with a 
frequency of $1 \mathrm{~Hz}$ is sampled at $0.2 \mathrm{~s}$, the striped line in Figure 2.6. The Fourier transform will not only retrieve the original $1 \mathrm{~Hz}$ wave, sampled at the diamonds in Figure 2.6, but can also fit a $4 \mathrm{~Hz}$ wave to those same points, see the red, solid line.

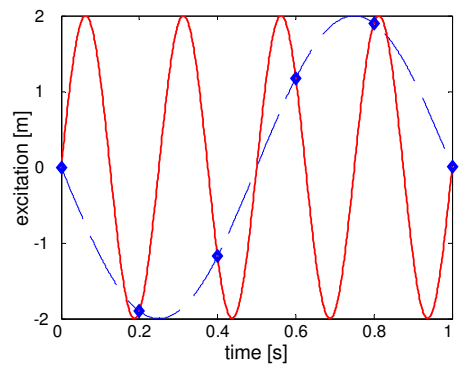

Figure 2.6 Aliasing effect: $1 \mathrm{~Hz}$ wave (striped line) is sampled at $0.2 \mathrm{~s}$ intervals (diamonds). FFT can also produce an incorrect but fitting $4 \mathrm{~Hz}$ wave to the same points (solid line)

To prevent this aliasing effect, a cut-off frequency must be defined, above which no frequencies should be included in the Fourier series. This so-called Nyquist frequency is defined by $f_{\text {Nyquist }}=1 /(2 \Delta t)[\mathrm{Hz}]$ with $\Delta t$ being the sample interval in [s]. Thus the highest frequency component of the frequency domain representation should be limited to $q=N / 2$.

A more everyday example of aliasing can be seen on TV when swiftly rotating objects are shown (helicopter rotor, wheels of a coach in a western). When speed varies, the object seems to rotate forward, backward or stand still, depending on the rotation speed and the reproduction speed of the television.

\section{Resonance peaks in response variables}

Fourier transforms can be applied to properties of natural phenomena (water surface elevation, wind speed) causing loads, but also to response records. The first category of signals is usually well behaved without sharp peaks in the outcome of the Fourier transform. However, response signals may contain sharp peaks at resonance frequencies, which require special attention during Fourier manipulations. The nature of these peaks is treated in section 2.7. If the frequency step $\Delta f$ is too big, the transformation could "step over" the crucial resonance frequency and miss its peak. To prevent this, the half power bandwidth $(h p b)$ of the peak can be determined from the natural frequency $f_{\text {nat }}$ and the damping coefficient $\beta$, from which $\Delta f$ should be chosen:

$$
\Delta f \leq h p b=2 \beta f_{\text {nat }}
$$

with:

\begin{tabular}{l|l|l}
\hline$\Delta f$ & frequency step & {$[\mathrm{Hz}]$} \\
$\beta$ & damping coefficient & {$[-]$} \\
$f_{\text {nat }}$ & natural frequency & {$[\mathrm{Hz}]$}
\end{tabular}


The requirement is that the frequency step is at least smaller than the $h p b$. For a better resolution of the spectrum a smaller frequency step is to be recommended: $\Delta f=0.25$ $h p b$. The requirements for successful Fourier Transformation are summarized in Figure 2.7 .

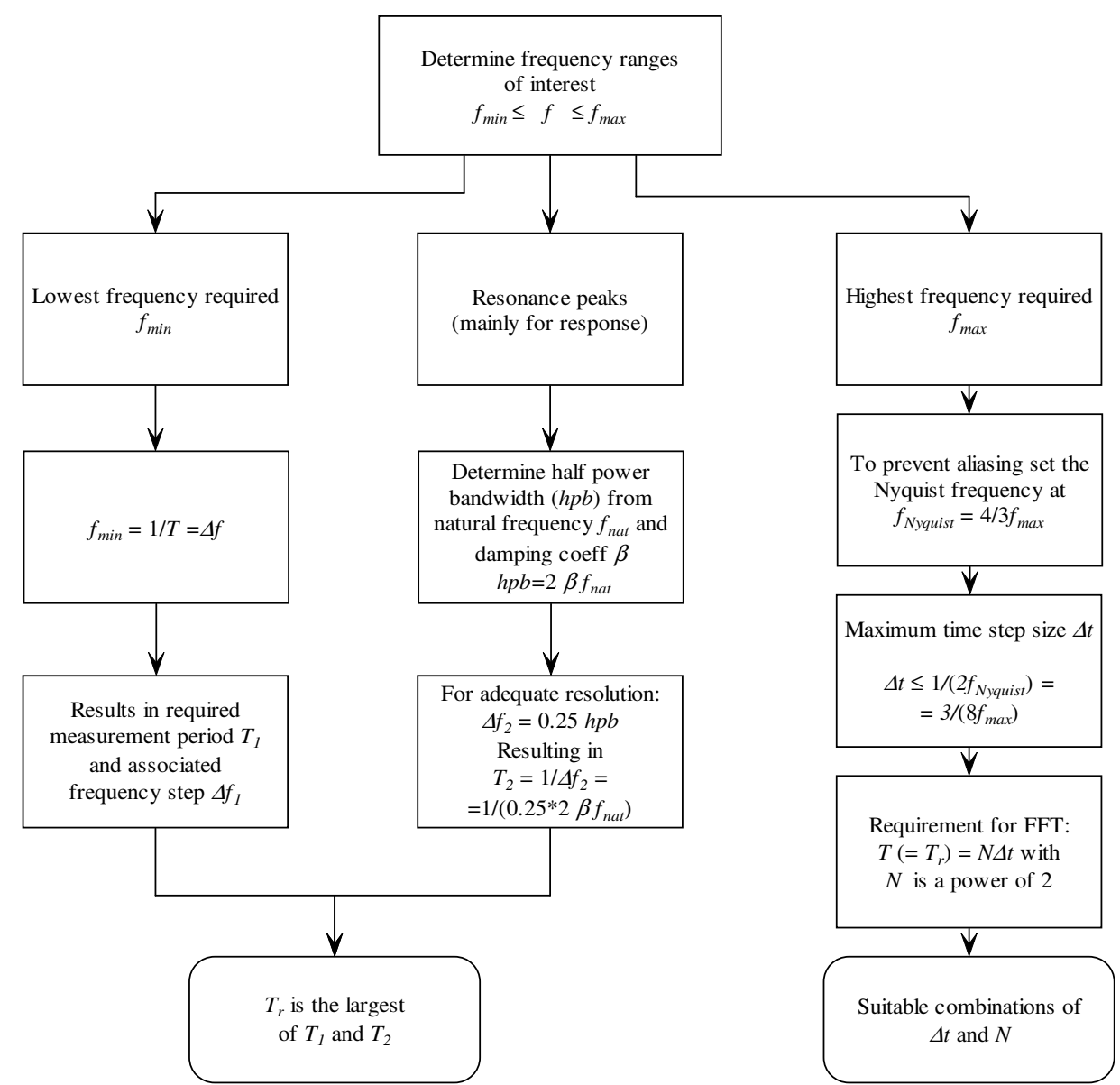

Figure 2.7 Requirements for the input time signal for successful Fourier transformation

Figure 2.7 illustrates in a diagram the procedure for selecting compatible values of the time step $\Delta t$, the number of data points $N$ and the basic record length $T_{r}$ to which the FFT can be successfully applied. $T_{r}$ is also called the subrecord length, hence the subscript $r . T_{r}$ is only one time record out of an infinite number of possible realisations of the random phenomenon of interest. To get a statistically reliable representation of 
this phenomenon a suitably large number $N_{F F T}$ of independent realisations, each of length $T_{r}$, need to be subjected to FFT analysis, making the total record $T=N_{F F T} T_{r}$.

\subsubsection{Trends and windowing of time series}

Not all signals have a zero mean; some even have a linearly deviating mean. Most spectral estimation algorithms give the option to remove the mean or trends. When effects are studied that are not influenced by a slowly deviating mean value, removing the trend will not have an effect on the analyses. But there are some cases where the trend is actually part of an even lower frequency contribution.

To produce spectra based on time series of finite length, the FFT procedure cuts the full time series into sub-records of length $T_{r}$. The "raw" spectral densities for all subrecords are then averaged to arrive at a "smoothed" spectrum. As discussed, the subrecord length is $T_{r}=N \Delta t$ with $N$ being a power of 2 to facilitate the FFT procedure. Examples for $N$ are $N=2^{10}=1024$ or $N=2^{11}=2048$ data points; this is the block size of the FFT analysis. A smaller block size reduces $T_{r}$ and therefore the frequency resolution making the spectrum smoother. However, this also reduces its capability to adequately capture sharp peaks. When a spectrum becomes too smooth, characteristic peaks may not be shown in the frequency domain representation of the time series data.

The discontinuities of the start and end of each finite length sub-record cause leakage of spectral density to adjacent frequencies. This so-called side-lobe leakage can be suppressed by applying a windowing technique: the actual signal is multiplied by a window, which reduces the ends smoothly to zero, suppressing the effects of the discontinuity. Figure 2.8 shows the use of a Hanning window or cosine taper. To compensate the influence of the windows on the Fourier transformation, the spectrum should be corrected by a factor $\sqrt{8 / 3}$.

The loss of sampled data due to the windowing can be compensated by using subrecords that have an overlap. The overlap can be up to $50 \%$, which means that with a number of $x x$ sub-records the total record length $T=x x T_{r}$ can be reduced to $T=(x x / 2) T_{r}$. Overlapping does mean that data is used twice, making the analyses no longer strictly independent. The overlapping is also shown in Figure 2.8. 
Original time series

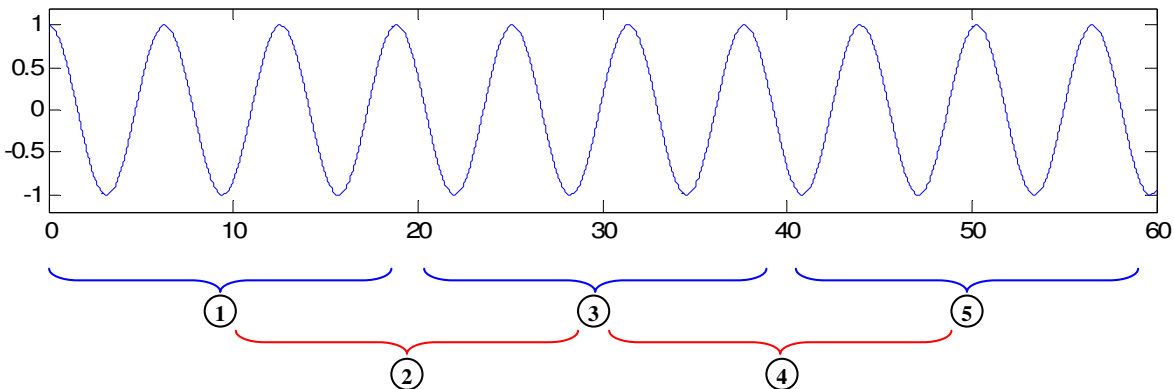

(1)

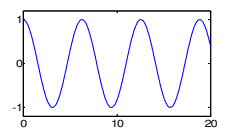

(2)

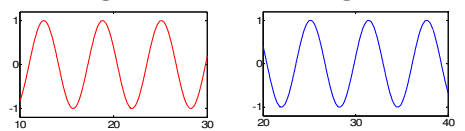

(4)
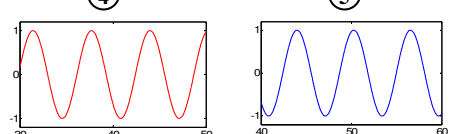

Apply window on each time record to minimize influence of finite length (here Hanning window)
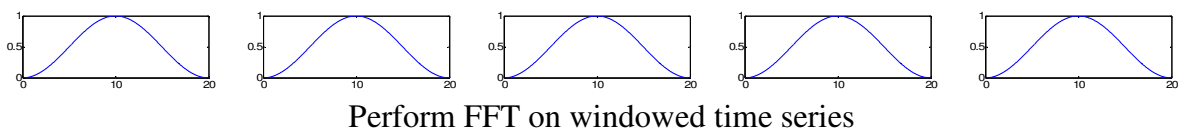

Perform FFT on windowed time series

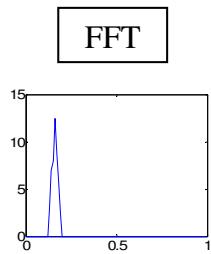

\section{FFT}

FFT
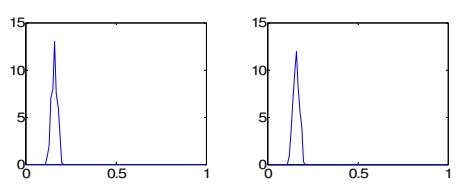

FFT

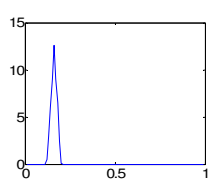

FFT

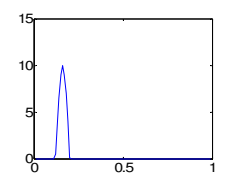

Take the mean of all spectra

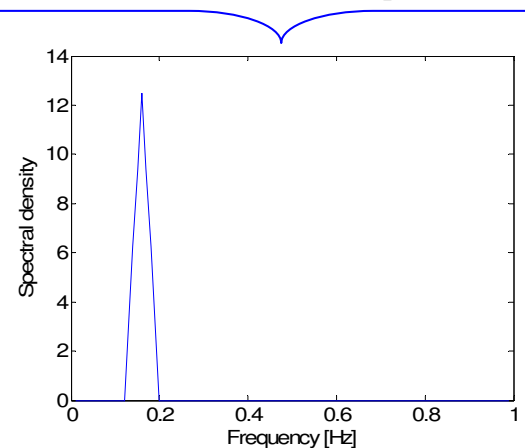

Figure 2.8 Windowing technique using a Hanning window and 50\% overlap of subrecords applied to a regular sine wave record 


\subsection{Waves and currents}

\subsubsection{Sea surface description}

The main driver behind sea waves is the wind. Only absolute absence of wind may result in a mirror-like sea surface, every slightest gust of wind will cause ripples, up to waves as large as houses. When examining the sea surface, numerous waves can be seen, travelling in, seemingly, every possible direction. To catch this random process, some models have been developed over the years.

If we reduce the surface of an area of sea to a single point, measuring the surface elevation in time as it runs through this point, the random sea in Figure 2.9a will result in the graph in Figure 2.9b. This time varying signal can be transformed to an energy density spectrum as shown in Figure 2.9c. This spectrum is also referred to as a wave spectrum.

a
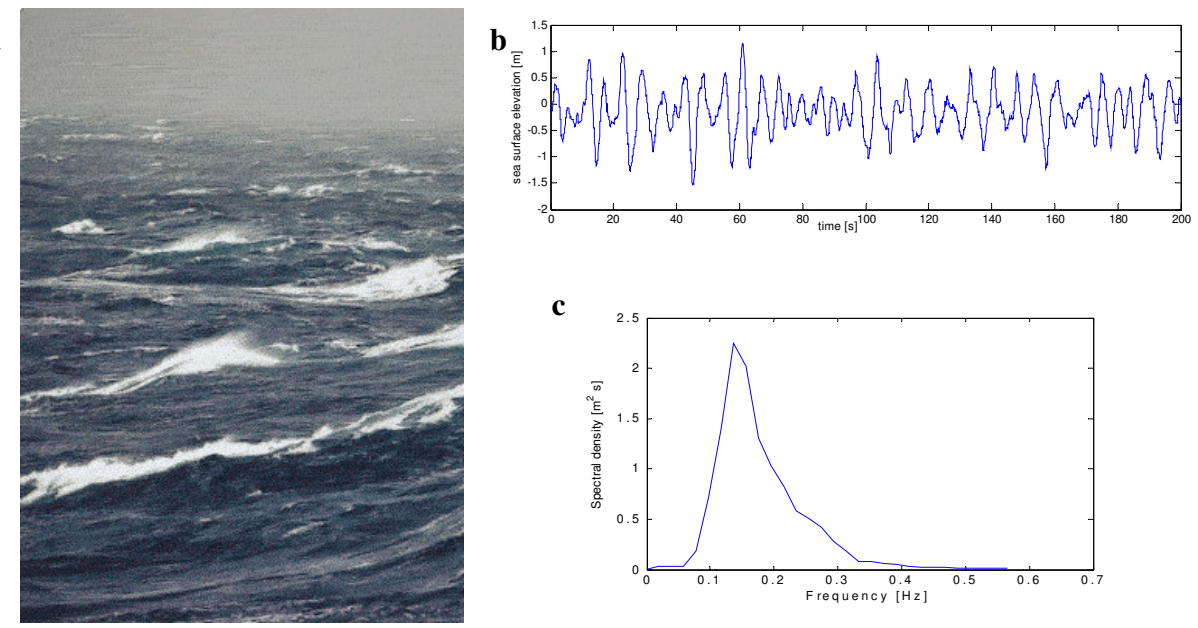

c

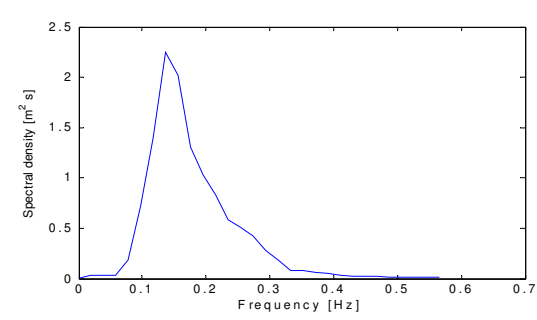

Figure 2.9 (a) Actual sea surface elevation. (b) Single point time recording of sea surface elevation. (c) Wave spectrum of measured time recording of sea surface elevation.

Some characteristic parameters can be defined from both the spectrum and the time series. The significant wave height of the spectrum, $H_{s}$, is defined as the mean of the $1 / 3$ highest waves in the time series, which is equal to 4 times the standard deviation $\sigma$ of the time series. The standard deviation is also the square root of the zeroth order moment of the spectrum, $m_{0}$. A remarkable feature of this parameter is that a visual estimate by sailors, $H_{v}$, compares rather well to the statistical $H_{s}$ value.

From the time series the mean zero crossing period $T_{z}$ can be found by dividing the measurement time by the number of zero up-crossings. This period can also be found 
from the spectrum as the square root of the zeroth moment over the second moment. A summary of the relations between time domain and spectral parameters is given in table 2.1. Note that these definitions are true for spectra based on frequency in [Hz] [7] [12].

Table 2.1. Relations between time series and spectral parameters for waves

\begin{tabular}{l|l} 
Description & Relation \\
\hline \hline spectral moments $(n=0,1,2 \ldots)$ & $m_{n}=\int_{0}^{\infty} f^{n} S(f) d f$ \\
variance or mean square & $\sigma^{2}=m_{0}$ \\
standard deviation or root-mean-square (RMS) & $\sigma=\sqrt{m_{0}}$ \\
significant wave height & $H_{s} \approx 4 \sigma$ \\
visual estimate of the wave height & $H_{v} \approx H_{s}$ \\
mean zero crossing period & $T_{z}=\sqrt{\frac{m_{0}}{m_{2}}}$ \\
mean period of the spectrum & $T_{m}=\frac{m_{0}}{m_{1}}$ \\
& \\
mean crest period & $T_{c}=\sqrt{\frac{m_{2}}{m_{4}}}$ \\
$\begin{array}{l}\text { estimate of the most probable maximum wave } \\
\text { height in a sea state for 1000 waves (appr. 3 hours) } \\
\text { for a Pierson-Moskowitz spectrum }\end{array}$ & $H_{\max }=1.86 H_{s}$
\end{tabular}

The shape of a wave spectrum has been fitted with several curves. A frequently used spectral shape is the Pierson-Moskowitz wave spectrum [8]. The shape was fitted to measurements taken in the Atlantic Ocean during long periods of constant environmental conditions. The spectrum is therefore based on the input of one single parameter: the average wind speed. The spectrum describes the sea surface elevation due to the wind speed for a fully developed sea at infinite fetch. Later, the spectrum description was adjusted to have $H_{s}$ and $T_{z}$ as input:

$$
S_{P M}(f)=\frac{H_{s}^{2}}{4 \pi T_{z}^{4} f^{5}} \exp \left(-\frac{1}{\pi}\left(f T_{z}\right)^{-4}\right)
$$

Figure 2.10 shows a plot of the Pierson-Moskowitz spectrum for the parameters $H_{s}$ and $T_{z}$ from the wave signal in figure 2.9. 


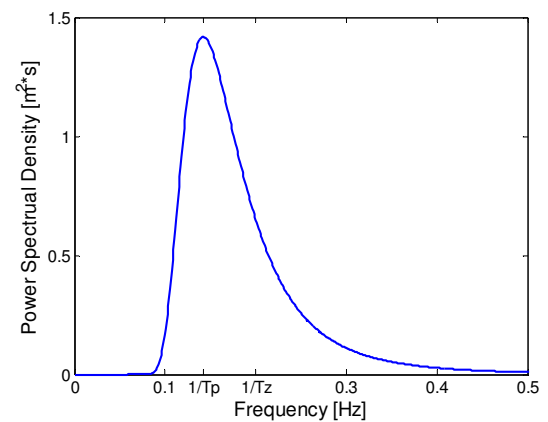

Figure 2.10 Pierson Moskowitz spectrum for $H_{s}=1.5 \mathrm{~m}$ and $T_{z}=5 \mathrm{~s}$ with mean zero-crossing frequency at $f_{z}=1 / T_{z}=0.2 \mathrm{~Hz}$ and peak spectral frequency at $f_{p}=0.14 \mathrm{~Hz}$

The mean of the zero-crossing frequency $f_{z}=1 / T_{z}$ is shown in the plot. A visually more characteristic parameter is the frequency at which the peak occurs, $f_{p}$, and its inverse $T_{p}$. Note that this peak refers to the peak of the spectrum and has nothing to do with the mean crest period $T_{c}$ referring to wave crests. The relation between $T_{p}$ and $T_{z}$ for the PM spectrum is $T_{p}=1.41 T_{z}$. Equation (2.7) can be re-written for $T_{p}$ :

$$
S_{P M}(f)=\frac{5}{16} \frac{H_{s}^{2}}{T_{p}^{4} f^{5}} \exp \left(-\frac{5}{4}\left(f T_{p}\right)^{-4}\right)
$$

The peak period becomes important for the enhancement of the Pierson-Moskowitz spectrum: further measurements of wave spectra were done in the Joint North Sea Wave Project from which the JONSWAP spectrum originated [9]. This spectrum represents sea states that are not fully developed under a certain wind condition. The wave spectrum shape is therefore much more peaked. The JONSWAP spectrum is actually an extended version of the Pierson-Moskowitz spectrum, incorporating a peak enhancement factor, which is controlled by a peak shape parameter $\gamma_{I S}$. When the shape parameter is taken as $\gamma_{I S}=1$ the JONSWAP spectrum is equal to the Pierson-Moskowitz spectrum. A typical value for not fully developed seas is $\gamma_{I S}=3.3$. The shape of the slopes before and after $T_{p}$, are controlled by slope factors $\sigma_{a}$ and $\sigma_{b}$.

Because the peak enhancement increases the total area under the spectrum, a normalising factor $F_{n}$ must be introduced to ensure that the area (the zeroth order moment) under the JONSWAP spectra still represents the real energy density of the sea state. Several derivations exist for this normalising factor; equation (2.9) shows one of the formulations from [12]. 


$$
\begin{gathered}
S_{J S}(f)=F_{n} \cdot S_{P M}(f) \cdot \gamma_{J S} \exp \left(\frac{-\left(f-f_{p}\right)^{2}}{2 \sigma_{J S} f_{p}^{2}}\right) \\
\sigma_{J S}=\left\{\begin{array}{l}
\left.\sigma_{a} \text { for } f \leq f_{p} \text { (typically: } 0.07\right) \\
\left.\sigma_{b} \text { for } f>f_{p} \text { (typically: } 0.09\right)
\end{array}\right. \\
F_{n}=\left[5\left(0.065 \gamma_{J S}^{0.803}+0.135\right)\right]^{-1} \text { for } 1 \leq \gamma_{J S} \leq 10
\end{gathered}
$$

Furthermore, the relation between $T_{p}$ and $T_{z}$ for a JONSWAP spectrum differs from that for a Pierson-Moskowitz spectrum. An approximation is shown in equation (2.10) [69]:

$$
T_{p} / T_{z} \approx 0.327 e^{-0.315 \gamma_{J S}}+1.17
$$

Using either the zero-crossing period or the peak period as basis for the wave spectrum, can result in different spectra. Figure 2.11 shows Pierson-Moskowitz and JONSWAP spectra for these two cases.
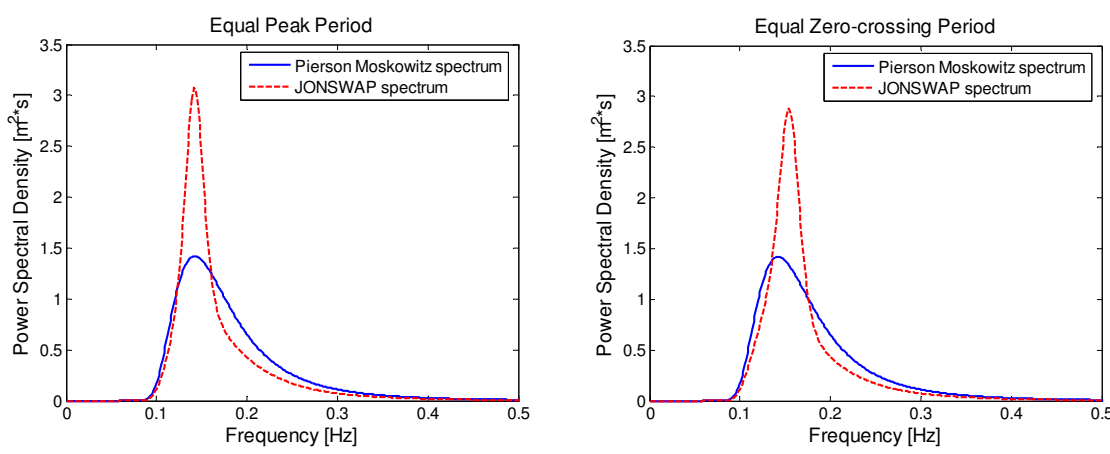

Figure 2.11 Pierson-Moskowitz (PM) and JONSWAP (JS) spectra for $H_{s}=1.5 \mathrm{~m}$ and $T_{z}=5 \mathrm{~s}$ left-hand side: equal peak period, right-hand side: equal zero-crossing period

Although Pierson-Moskowitz and JONSWAP are the most commonly used spectra, other descriptions exist. Remember that all these "standard" spectra try to reproduce the actual measured spectra at a certain location under certain circumstances. When detailed, long-term measurements are available for a site, more finely tuned spectra can be used. In all other cases the designer can only select an appropriate shape based on the parameters available for a site. 


\subsubsection{Representation of a random sea}

With a suitable wave spectrum, the calculations can start by converting the spectrum back into individual sinusoids with IFFT. The sinusoids have an amplitude and a frequency that can be derived from the energy density given by the spectrum. The phase angle is appointed randomly to each sinusoid. The sum of all these harmonic waves is the sea surface elevation at each point in time.

For every harmonic wave, the water particle motion can be described by linear wave theory according to Airy [12]. In deep water the water particles are moving in circles in accordance with the harmonic wave. The diameter of the circle decays with depth below the surface. When the water depth becomes small relative to the wavelength, $\lambda_{\text {wave }}<0.5 d$, the effect from the seabed transforms the circular motion into an elliptic motion. The particle motion is shown in Figure 2.12.

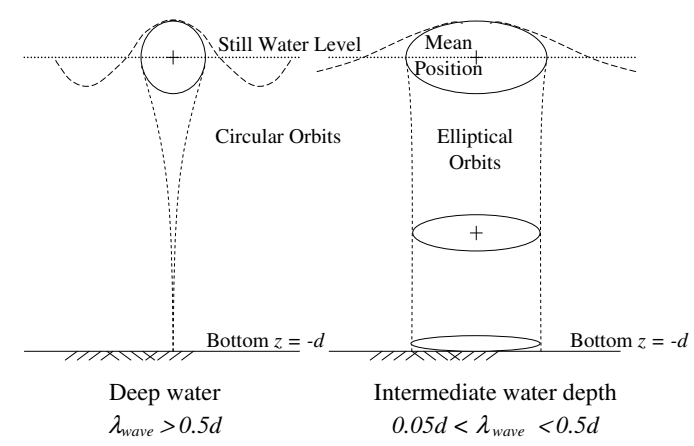

Figure 2.12 Particle orbits according to the Airy theory

The horizontal water particle kinematics are described by equation (2.11), with the $z$-axis pointing upwards from the free water surface $(-d \leq z \leq 0)$ and position $x$ horizontally in the wave direction; $t$ is time:

$$
\begin{aligned}
& u(x, z ; t)=\hat{\zeta} 2 \pi f \frac{\cosh k_{\text {wave }}(z+d)}{\sinh k_{\text {wave }} d} \cos \left(k_{\text {wave }} x-2 \pi f t\right) \\
& \dot{u}(x, z ; t)=\hat{\zeta}(2 \pi f)^{2} \frac{\cosh k_{\text {wave }}(z+d)}{\sinh k_{\text {wave }} d} \sin \left(k_{\text {wave }} x-2 \pi f t\right)
\end{aligned}
$$

with:

\begin{tabular}{l|l|l}
\hline$\hat{\zeta}$ & wave amplitude $(=0.5 H)$ & {$[\mathrm{m}]$} \\
$k_{\text {wave }}$ & wave number $=2 \pi / \lambda_{\text {wave }}$ & {$\left[\mathrm{m}^{-1}\right]$} \\
$f$ & wave frequency & {$[\mathrm{Hz}]$} \\
$\lambda_{\text {wave }}$ & wavelength & {$[\mathrm{m}]$} \\
$d$ & water depth & {$[\mathrm{m}]$}
\end{tabular}


Because Airy linear wave theory is only valid up to the still water level, it does not describe the kinematics in the wave top. Several methods have been suggested to correct this. One of the most commonly used methods is Wheeler stretching [11] [12]. The wave kinematics profile over the water depth is determined for every time step. This profile is then stretched to the instantaneous wave surface elevation. The method is shown in Figure 2.13.

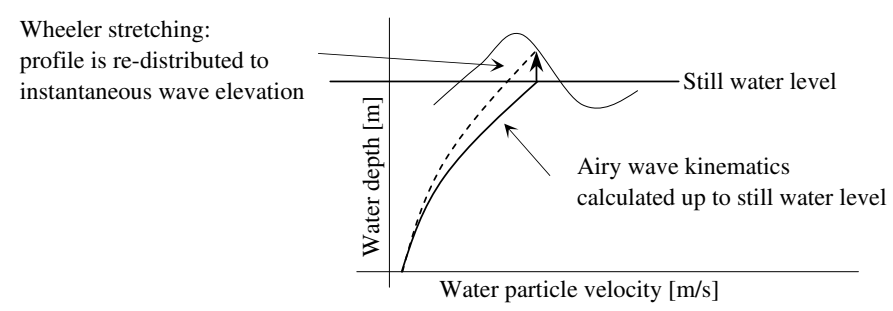

Figure 2.13 Normal Airy wave kinematics up to the still water level and Wheeler stretching to the instantaneous wave elevation

\subsubsection{Wave loads on structures}

\section{Loads on vertical towers}

The wave particle kinematics can now be used to calculate the loads on a structure with the Morison Equation. The relative velocity of the structure can also be incorporated but is ignored here as its magnitude is very small compared to the water particle velocities. The Morison equation is an empirical formula to calculate the hydrodynamic loads on slender members per unit length:

$$
\begin{aligned}
& f_{\text {Morison }}(x, z, t)=f_{d}(x, z, t)+f_{i}(x, z, t) \\
& f_{d}(x, z, t)=C_{d} \cdot \frac{1}{2} \rho_{\text {water }} D \cdot|u(x, z, t)| u(x, z, t) \\
& f_{i}(x, z, t)=C_{m} \cdot \frac{\rho_{\text {water }} \pi D^{2}}{4} \cdot \dot{u}(x, z, t)
\end{aligned}
$$

with:

\begin{tabular}{l|l|l}
\hline$f_{\text {Morison }}$ & hydrodynamic load & {$[\mathrm{N} / \mathrm{m}]$} \\
$f_{d}$ & hydrodynamic drag load & {$[\mathrm{N} / \mathrm{m}]$} \\
$f_{i}$ & hydrodynamic inertia load & {$[\mathrm{N} / \mathrm{m}]$} \\
$C_{d}$ & hydrodynamic drag coefficient & {$[-]$} \\
$C_{m}$ & hydrodynamic inertia coefficient & {$[-]$} \\
$\rho_{\text {water }}$ & density of water & {$\left[\mathrm{kg} / \mathrm{m}^{3}\right]$} \\
$u$ & water particle velocity & {$[\mathrm{m} / \mathrm{s}]$} \\
$\dot{u}$ & water particle acceleration & {$\left[\mathrm{m} / \mathrm{s}^{2}\right]$} \\
$D$ & diameter of cylinder section & {$[\mathrm{m}]$}
\end{tabular}


Figure 2.14 shows the representation of a slender vertical member under hydrodynamic loads. The sum of drag and inertia load is the total hydrodynamic load on the cylinder. Note that velocity and acceleration have a $90^{\circ}$ phase difference, so inertia and drag loads will also be out of phase. This means that in general the maximum load is not equal to either maximum drag or maximum inertia load.

The coefficients $C_{m}$ and $C_{d}$ are determined by the specific load case under consideration (extreme or fatigue), the shape of the structure, the presence of marine growth and other factors. Typical values for fatigue calculations on monopile structures are $C_{m}=2.0$ and $C_{d}=0.7$, but the designer has to make sure the values are correct for each specific load case [12].

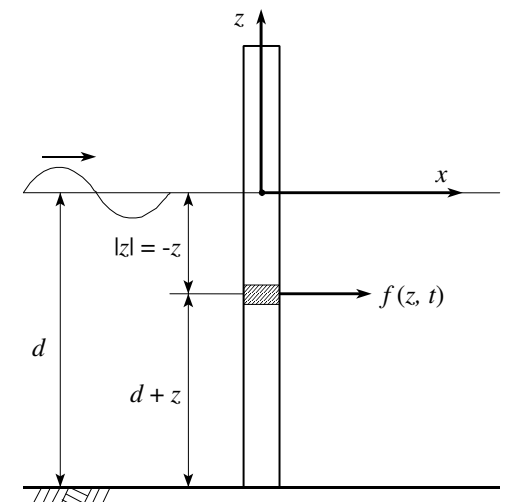

Figure 2.14 Slender vertical tower with hydrodynamic loads

\section{Horizontal and inclined members}

When members of a structure are inclined or horizontal, the Morison equation can still be used, but the amount of administration increases significantly. Not only the horizontal velocities and accelerations then need to be calculated per structural interval, with the Airy theory, but the velocity and acceleration vectors in the horizontal and the vertical directions must be transposed to those perpendicular to the member under consideration. The overall structural loading can then be found by combining all individual section loads in their local grid in relation to the global grid.

Next to the bookkeeping required for the different inclinations of structural members, also a phase shift occurs as a wave top first reaches one leg of a structure and some time later the next. Although the amount of calculations required for more complex structures increases significantly when compared to a single vertical tower, computer programs can very effectively handle all this computational administration. 


\section{Adding current}

When a structure is not only subjected to wave particle velocity and acceleration, but also to a current, the current velocity must be incorporated in the calculation of the total hydrodynamic load in the Morison equation. As the velocity is only a parameter in the drag term, the combined wave and current drag load equation will be:

$$
f_{d}=C_{d} \cdot \frac{1}{2} \rho_{\text {water }} D \cdot\left(\left|\left(u+U_{c}\right)\right|\left(u+U_{c}\right)\right)
$$

with:

\begin{tabular}{l|l|l}
$u$ & wave induced particle velocity & {$[\mathrm{m} / \mathrm{s}]$} \\
$U_{c}$ & current velocity & {$[\mathrm{m} / \mathrm{s}]$}
\end{tabular}

\section{Diffraction}

The basic assumption of the Morison equation is that the submerged members on which the wave loads are calculated do not affect the waves. As long as the cylinder diameter is relatively small compared to the wave length this assumption is valid. For large diameter structures, like the monopile support structures for offshore wind turbines, placed in relatively shallow water with consequently reduced wave length, the validity of the Morison equation can be compromised.

The effect a structure has on the wave field is called diffraction. To incorporate this effect in the Morison equation, the MacCamy-Fuchs correction is introduced [10]. This correction reduces the magnitude of the inertia coefficient. The correction factor is dependent on the ratio of diameter over wave length: $D / \lambda_{\text {wave }}$. Figure 2.15 shows the reduction of $C_{m}$ for increasing ratio.

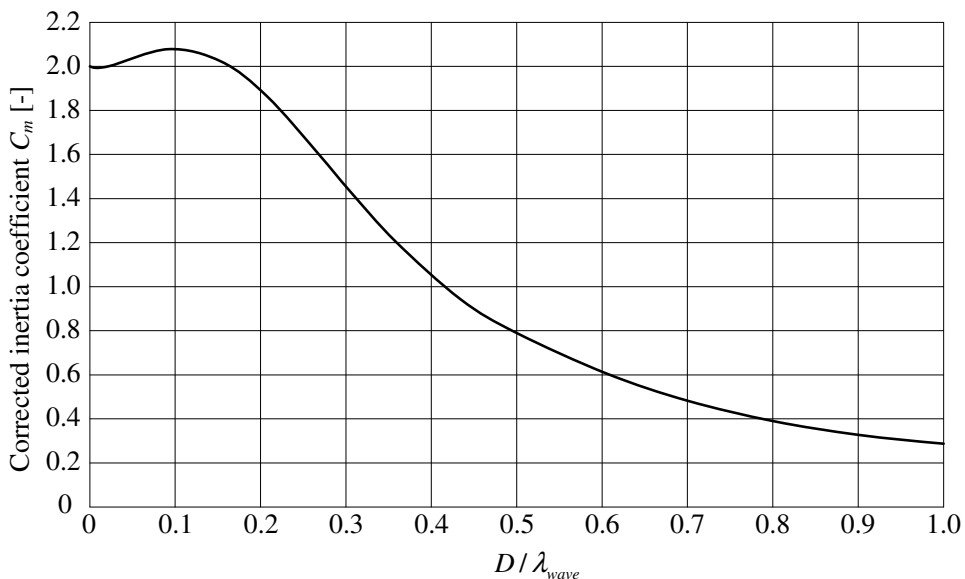

Figure 2.15 MacCamy-Fuchs diffraction correction of the inertia coefficient $C_{m}$ in the Morison equation for cylinder diameter over wave length 


\subsubsection{Extreme waves, non-linear wave theories and breaking waves}

To determine the extreme wave loads on a structure, the maximum wave for the site must be known. When long-term measurements of wave heights are available, a distribution curve can be fitted through the maximum values and extrapolated to find the 50 and 100 year return period maximum wave height. It is also possible that the maximum wave height is limited by to the breaking wave limit due to reduced water depth at the site or in the vicinity (sand banks).

To calculate the wave forces on the structure, the linear Airy theory does not always completely describe the more non-linear features of these extreme waves. Figure 2.16 shows which theory to use in which situation based on the relation between wave height, period and water depth. As the non-linear methods can only be used to model deterministic waves, they are not suitable to model stochastic wave fields.

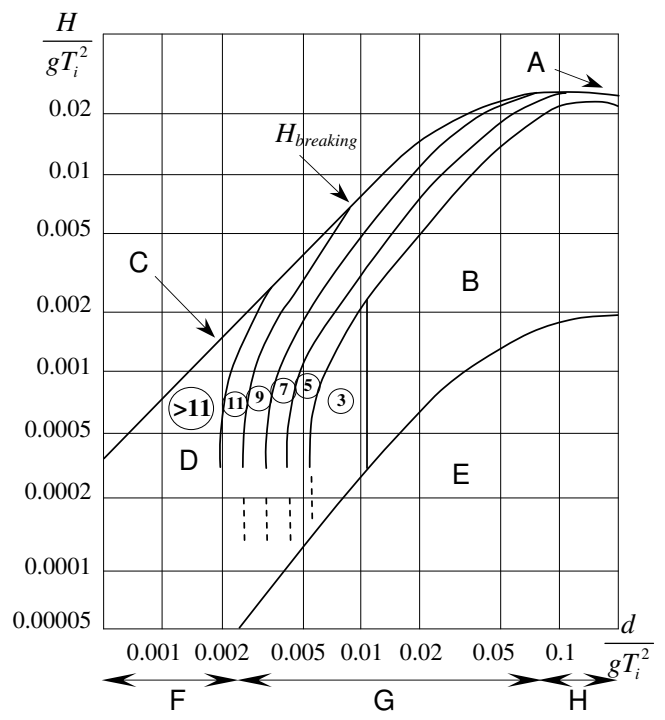

A Deep water breaking limit $H / \lambda_{\text {wave }}=0.14$

B Stokes' 5th order, New Wave or 3rd order Stream Function

C Shallow water breaking limit $H / d=0,78$

D Stream function (showing order number)

E Linear / Airy or 3rd order Stream Function F Shallow water

G Intermediate depth

H Deep water

Figure 2.16 Regions of applicability of different wave theories [12]

The area of applicable theories is enveloped by the breaking wave limit. Waves are assumed to break when $H / d>0.78$. The probability of breaking waves at a specific offshore wind farm site needs to be assessed. At sites with a steep sea floor slope, at sandbanks or rocky outcrops, the probability of breaking waves is high. This is for instance the case at Blyth. Because wave breaking directly onto the pile will induce large loads on the structure, more detailed analysis may be required in such situations. 
Although many experiments have been conducted to find a suitable empirical formulation [13] [14] for the loads of breaking waves on structures, much uncertainty remains. DNV [19] prescribes equation (2.14) to calculate the breaking wave load:

$$
F=1 / 2 \rho_{\text {water }} C_{S} A u^{2}
$$

with:

\begin{tabular}{l|l|l}
\hline$C_{s}$ & slamming coefficient (range: 2 - 6.3) & {$[-]$} \\
$\rho_{\text {water }}$ & water density & {$\left[\mathrm{kg} / \mathrm{m}^{3}\right]$} \\
$A$ & area exposed to breaking wave & {$[\mathrm{m} / \mathrm{s}]$} \\
$u$ & water particle velocity in breaking wave crest & {$[\mathrm{m}]$}
\end{tabular}

Fortunately, the probability of breaking waves is relatively small on a nearly horizontal seabed without abnormal bathymetrical characteristics: most waves just break at or near the coast and not within the offshore wind farm.

\subsubsection{Long-term wave description}

All wave events discussed in the previous sections dealt with sea conditions that are assumed stationary for a certain period of time. The wave conditions during these periods are called sea states and they typically last 3 hours. When long-term effects of the wave climate at a certain site are studied to find the probability of extremes or to calculate the lifetime fatigue damage, a large number of data is required. These data can be taken by measurements from buoys, platforms or satellites, or can be estimated from large meteorological and oceanographic models fed and calibrated with measurements from a large number of stations. The resulting sea states are binned into a scatter diagram. This diagram gives the probability of the occurrence of each combination of $H_{s}$ and $T_{z}$ for that location. Combinations of $H_{s}$ and $T_{p}$ are also common. Scatter diagrams based on different wave periods should not be confused, see section 2.4.1. Every bin represents the probability of occurrence of wave conditions having that specific combination of parameter values, with which the wave spectrum according to a selected spectral model can be determined. The size of the bins can be selected arbitrarily: larger bins will remove a lot of detail but reduce the total number of sea states. A typical bin size for $H_{s}$ is steps of $0.5 \mathrm{~m}$ and for $T_{z}$ (or $T_{p}$ ) steps of $2 \mathrm{~s}$.

When looking at the cloud of occurrences in a wave scatter diagram, the image arises that there is some more or less linear relationship hidden in the cloud of occurrences: the cloud runs from bottom-left to top-right. The general direction of this cloud is of course trivial: large waves have large periods (or they become too steep and break) and small waves have smaller periods. But to fix a linear relationship would considerably reduce the variability reflected in the diagram and discard valuable information. 
Because scatter diagrams are very site-specific, it is even more dangerous to superimpose the assumed linear relationships of one location onto the next. Figure 2.17 shows the scatter diagram for the OWEZ or "NL1" location [5].

\begin{tabular}{|c|c|c|c|c|c|c|c|c|c|}
\hline$T_{z}[\mathrm{~s}]$ & \multirow{2}{*}{$0-1$} & \multirow{2}{*}{$1-2$} & \multirow{2}{*}{$2-3$} & \multirow{2}{*}{$3-4$} & \multirow{2}{*}{$4-5$} & \multirow{2}{*}{$5-6$} & \multirow{2}{*}{$6-7$} & \multirow{2}{*}{$7-8$} & \multirow{2}{*}{ Sum: } \\
\hline$H_{s}[\mathrm{~m}]$ & & & & & & & & & \\
\hline $6.5-7.0$ & & & & & & & & & $\mathbf{0 . 0}$ \\
\hline $6.0-6.5$ & & & & & & & & 0.1 & 0.1 \\
\hline $5.5-6.0$ & & & & & & & 0.1 & 0.1 & 0.2 \\
\hline $5.0-5.5$ & & & & & & & 0.1 & 0.1 & 0.2 \\
\hline 4.5-5.0 & & & & & & & 1 & & 1.0 \\
\hline $4.0-4.5$ & & & & & & & 4 & & 4.0 \\
\hline $3.5-4.0$ & & & & & & 4 & 5 & & 9.0 \\
\hline $3.0-3.5$ & & & & & & 19 & 0.1 & & 19.1 \\
\hline $2.5-3.0$ & & & & & 0.1 & 38 & & & 38.1 \\
\hline $2.0-2.5$ & & & & & 27 & 43 & & & 70.0 \\
\hline $1.5-2.0$ & & & & 0.1 & 115 & 5 & & & 120.1 \\
\hline 1.0-1.5 & & & & 6 & 220 & 1 & & & 227.0 \\
\hline $0.5-1.0$ & & & & 236 & 145 & 1 & & & 382.0 \\
\hline $0.0-0.5$ & 1 & & 1 & 113 & 14 & 0.1 & & & 129.2 \\
\hline Sum: & 1.0 & 0.0 & 1.0 & 355.1 & 521.1 & 111.1 & 10.4 & 0.3 & 1000 \\
\hline
\end{tabular}

Figure 2.17 Wave scatter diagram for $H_{s}$ and $T_{z}$ with occurrence in parts per thousand for the OWEZ location [5]

\subsubsection{Sea level elevation: tides}

The mean sea level elevation varies in time. The main driver of these variations is the tide, but also storm surges may temporarily raise or lower the mean elevation. Tides are driven by the gravitational pull of the moon and sun. This pull creates two bulges of water on both sides of the globe, underneath which the earth rotates. This results in 2 high and 2 low tides every 24 hours for most locations. These high and low tides are further influenced by the local bathymetry. For basin-like areas like the North Sea, the tidal wave is trapped and rotates within the basin, creating an amphidromic system. The tidal wave rotates within the basin and is driven by the Coriolis force due to the earth's rotation, which gives it a counter clock-wise rotation on the Northern Hemisphere. The tidal wave rotates around amphidromic points where the tidal range is zero. Further away from these points the tidal range increases. Figure 2.18 shows the tidal ranges in the North Sea with amphidromic points; medium tidal ranges of nearly $2 \mathrm{~m}$ occur at the Dutch coast, more than $4 \mathrm{~m}$ at the British East coast and large ranges of up to $12 \mathrm{~m}$ occur in the English Channel. The latter tidal range is also driven by the funnelling effect of the local bathymetry. 


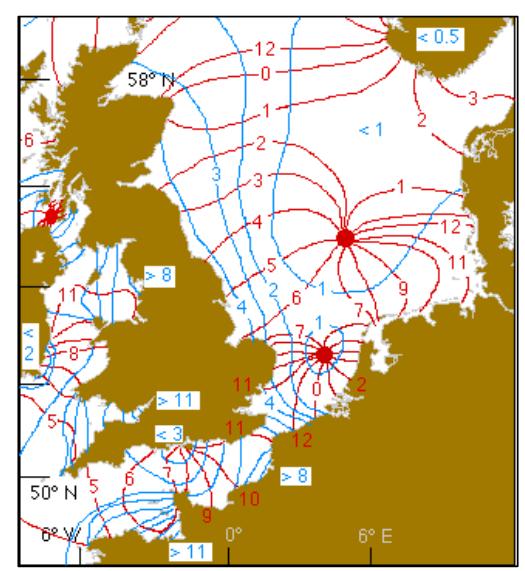

Figure 2.18 Tidal ranges in the North Sea. Blue lines: co-range line, locations with the same tidal range, red lines: co-tidal lines: locations that experience the tide at the same time [15]

The tidal range also has monthly fluctuations. When the sun and moon are aligned with each other, at full moon and new moon, the tidal bulges become extra large, causing a spring tide with higher high-water and lower low-water. When sun and moon are oriented under a $90^{\circ}$ angle of each other, the tidal range is smaller than usual, a neap tide. For design considerations, the spring tides are usually critical for the height of the boat landing, and they can affect the extreme load calculations. For installation matters, the range of the neap tide can be interesting: installation of gravity based structures is easier at neap tide due to the longer period of constant water level and thus minimum currents.

Sea map water depths are usually related to the mean of the lowest astronomical tide (LAT). This means that sailors always know the minimum water depth at locations on the map.

\subsubsection{Currents}

Sea currents are mostly driven by the tides and ocean circulations, although the outflow of rivers, differences in temperature or salinity and storm surges may cause extra local currents. The currents are usually largest where large tidal differences occur, or where local bathymetry influences the tidal currents drastically. An example of the latter is Horns Rev, where the tidal wave of the North Sea is pushed around the corner of Blåvands Huk. This regularly causes local surface current velocities of $2 \mathrm{~m} / \mathrm{s}$.

The current velocity variation is of a time scale much larger than the time scales for load variations associated with the design of offshore wind turbines. It is therefore common to assume the instantaneous surface current velocity and direction to be 
constant in design calculations. The variation of this velocity over water depth is then the only parameter to define. Again local effects have influence on this variation, which can only be established by on-site measurements or expert analysis. When these are not readily available, the design guides usually present three basic current profiles over depth:

- the linear profile

- the bilinear profile

- the power law profile, equation (2.15):

$$
U_{c}(z)=U_{c 0}\left(\frac{z+d}{d}\right)^{\alpha_{c u r}} \quad(-d \leq z \leq 0)
$$

with:

\begin{tabular}{l|l|l}
$U_{c}(z)$ & current velocity at elevation $z$ & {$[\mathrm{~m} / \mathrm{s}]$} \\
$U_{c 0}$ & current velocity at the sea surface $z=0$ & {$[\mathrm{~m} / \mathrm{s}]$} \\
$z$ & vertical co-ordinate, measured positively upwards from mean sea level & {$[\mathrm{m}]$} \\
$d$ & mean water depth & {$[\mathrm{m}]$} \\
$\alpha_{c u r}$ & current exponent, usually $1 / 7$ & {$[-]$}
\end{tabular}

The profiles are shown in Figure 2.19.

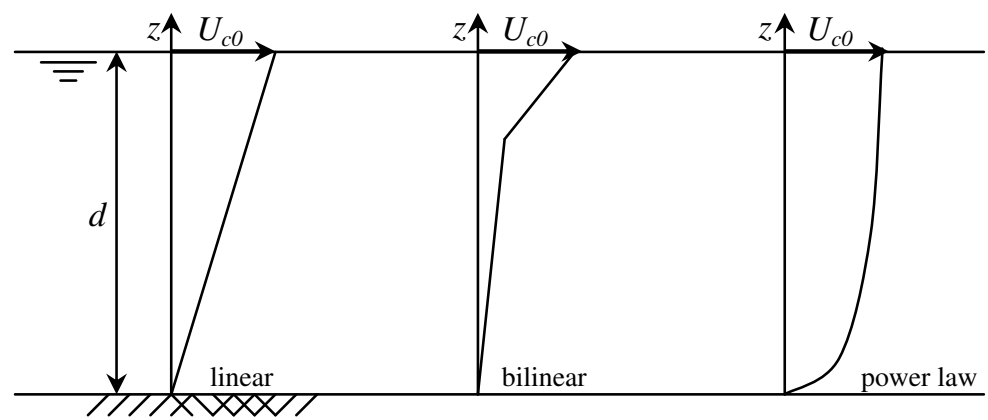

Figure 2.19 Current profile over water depth: linear, bilinear and power law

For currents in combination with waves, the current profile should be stretched analogous to the stretching of waves described in 2.4.2; see reference [12]. 


\subsection{Wind}

\subsubsection{Wind force and wind speed}

Measuring the intensity of the wind first originated from marine applications: in 1838 Admiral Beaufort of the Royal Navy created a look-up table to relate wind force to ship behaviour. Dividing the wind force in 13 classes (0-12) proved an effective method to $\log$ wind behaviour throughout the British fleet. Even the introduction of wind speed measurement with anemometers did not make the Beaufort scale obsolete. The fact that the Navy was the instigator of early meteorological institutions made Beaufort a logical scale to adopt; also the ease to communicate the scale via Morse code prolonged its use. To correlate the wind force scale to real, measurable wind velocity, the World Meteorological Organisation (WMO) eventually drafted a standard table. The Beaufort scale is shown in table 2.2 with speeds in knots and $\mathrm{m} / \mathrm{s}$.

Table 2.2. Beaufort scale with equivalent wind speed and WMO description [16]

\begin{tabular}{c|c|c|l} 
Beaufort & Knots & $\mathrm{m} / \mathrm{s}$ & WMO description \\
\hline \hline 0 & $<1$ & $<0.2$ & Calm \\
1 & $1-3$ & $0.3-1.5$ & Light air \\
2 & $4-6$ & $1.6-3.3$ & Light breeze \\
3 & $7-10$ & $3.4-5.4$ & Gentle breeze \\
4 & $11-16$ & $5.5-7.9$ & Moderate breeze \\
5 & $17-21$ & $8.0-10.7$ & Fresh breeze \\
6 & $22-27$ & $10.8-13.8$ & Strong breeze \\
7 & $28-33$ & $13.9-17.1$ & Near gale \\
8 & $34-40$ & $17.2-20.7$ & Gale \\
9 & $21-47$ & $20.8-24.4$ & Strong gale \\
10 & $48-55$ & $24.5-28.4$ & Storm \\
11 & $56-63$ & $28.5-32.6$ & Violent storm \\
12 & $>64$ & $>32.7$ & Hurricane
\end{tabular}

The current generation of wind turbines usually start operating at 3-4 m/s wind speed and will shut down at around $25 \mathrm{~m} / \mathrm{s}$. In Beaufort terms this would be an operational window between scale 3 and 10 .

Though the Beaufort scale is still used in meteorological forecasts and by the man-inthe-street, in wind energy technology it is common to address wind motion as wind speed in metres per second. Because the wind speed is constantly changing, the main feature of wind speed is its mean. Either over short intervals called gusts (3-10 s), or as 10 minute means, daily means, monthly means or yearly means.

When taking a longer measurement period, the time varying character of the wind can be captured in a wind spectrum, covering frequency ranges from years to seconds. This spectrum, after Van der Hoven [17], is shown in Figure 2.20. 


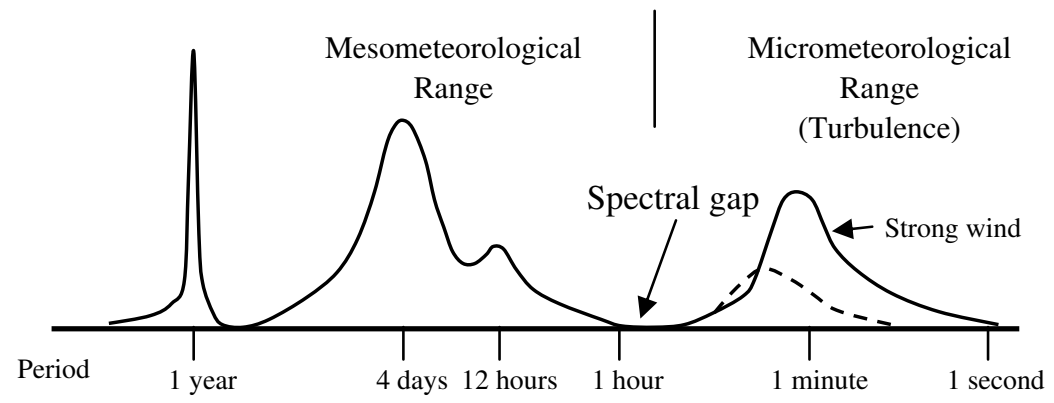

Figure 2.20 Wind speed spectrum over a broad range of frequencies [17]

The frequency content on the left hand side represents the yearly changes, pressure systems and diurnal changes. On the right hand side, the turbulence is visible. The solid line represents high turbulence during a period of high wind speeds, the dotted line reduced turbulence at lower wind speeds. The details of turbulence will be described in the next section. The final characteristic to note is the gap around the 1-hour period, which is known as the "spectral gap", which separates the slowly changing and turbulent ranges. Because in this frequency range the wind speed does not change, the mean over a period of 10 minutes to 1 hour can be considered constant: the instantaneous wind speed changes with turbulence, but the mean wind speed stays constant over the interval.

Recent research on the spectral gap revealed that the gap was more a coincidental feature of the analysis technique Van der Hoven used to create the plot from short term and long term measurements [18]. Although this is probably true, the assumption that in the 10 minute to 1 hour range the mean wind speed is constant has proved to be an effective model for wind turbine design in the past and will therefore be adhered to in this thesis.

\subsubsection{Wind shear and turbulence}

\section{Introduction}

The wind velocity measured in the field shows variations in space, time and direction. A momentary representation of a typical wind speed distribution is shown in Figure 2.21 . 


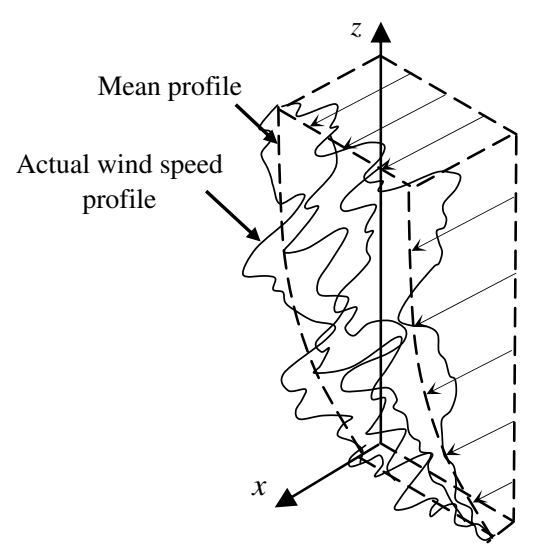

Figure 2.21 Actual wind speed profile

From the figure it is clear that the mean wind speed increases with height, a phenomenon called wind shear. Furthermore, the actual wind speed at any location varies in time and direction around its mean value due to the effect of turbulence.

\section{Wind shear}

In the lower $2 \mathrm{~km}$ of the earth's atmosphere, the atmospheric boundary layer, the wind speed is affected by friction with the earth's surface. This effect, known as wind shear, reduces the wind speed from its undisturbed value at $2 \mathrm{~km}$ to nearly zero at the surface. To describe the shear effect on the mean wind speed at a certain elevation, two main models are commonly used: the logarithmic profile and the power law profile. Both profiles are fitted curves to measured wind shear effects. The logarithmic profile and the power law profile are described by equations (2.16)a, b:

(a)

$$
V_{w}(z)=V_{w, r} \cdot \frac{\ln \left(\frac{z}{z_{0}}\right)}{\ln \left(\frac{z_{r}}{z_{0}}\right)}
$$

(b)

$$
V_{w}(z)=V_{w, r}\left(\frac{z}{z_{r}}\right)^{\alpha_{\text {shear }}}
$$

with:

\begin{tabular}{l|l|l}
$V_{w}(z)$ & mean wind speed at height $z$ & {$[\mathrm{~m} / \mathrm{s}]$} \\
$V_{w, r}$ & mean wind speed at the reference height $z_{r}$ & {$[\mathrm{~m} / \mathrm{s}]$} \\
$z_{r}$ & reference height & {$[\mathrm{m}]$} \\
$z_{0}$ & surface roughness length & {$[\mathrm{m}]$} \\
$\alpha_{\text {shear }}$ & power law coefficient & {$[-]$}
\end{tabular}


Typical values for the surface roughness length for different types of terrain are given in table 2.3. DNV mentions the use of $z_{0}=0.05 \mathrm{~m}$ for offshore [19], unless stated differently. GL advises in their offshore wind regulations to use $z_{0}=0.002 \mathrm{~m} \mathrm{[20]}$.

Table 2.3 Roughness length and power law factor for different types of terrain [21]

\begin{tabular}{l|c|c} 
Type of terrain & $z_{0}[\mathrm{~m}]$ & $\alpha_{\text {shear }}$ \\
\hline \hline City centres & $1-10$ & 0.40 \\
Cities, forests & 0.7 & \\
Suburbs, wooded country side & 0.3 & 0.30 \\
Villages, countryside with trees and hedges & 0.1 & \\
Open farmland, few trees and buildings & 0.03 & 0.16 \\
Flat grassy plains & 0.01 & \\
Flat desert, rough sea & 0.001 & 0.12 \\
Calm sea & 0.0002 &
\end{tabular}

A comparison of the log and power law profiles is shown in Figure 2.22. Figure 2.22a shows the $\log$ and power law curves for a mean wind speed of $10 \mathrm{~m} / \mathrm{s}$ at a reference height of $10 \mathrm{~m}$. Some data sources have reference heights of $60 \mathrm{~m}$. Figure $2.22 \mathrm{~b}$ shows a plot for the latter case and a mean wind speed of $12 \mathrm{~m} / \mathrm{s}$.

a

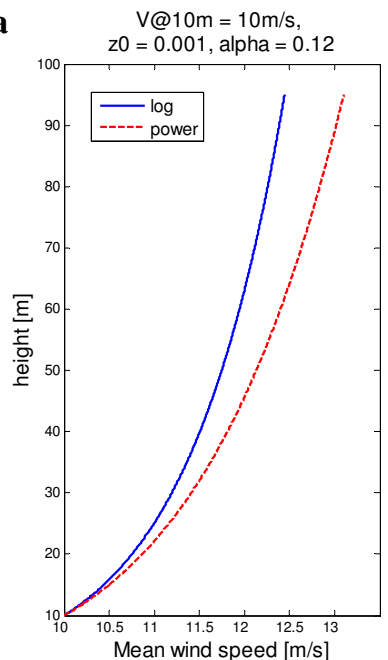

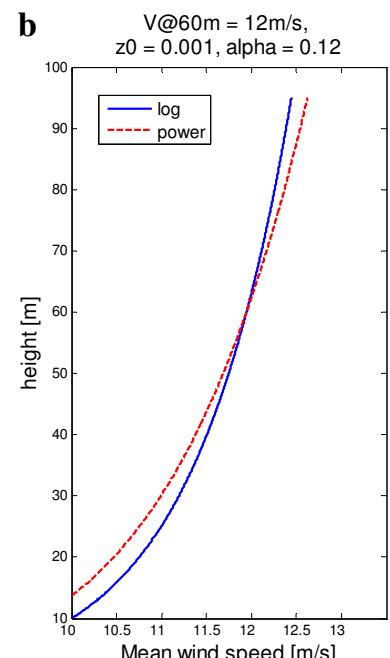

Figure 2.22 Wind shear according to log and power law model a) mean wind speed at $10 \mathrm{~m}$ of $10 \mathrm{~m} / \mathrm{s}$ b) mean wind speed at $60 \mathrm{~m}$ of $12 \mathrm{~m} / \mathrm{s}$ 
Figure 2.22 clearly shows that when a data source is used with wind measurements at $10 \mathrm{~m}$ height, the theories differ more and more as the hub height increases. Before or during the first stages of design the parties should agree on which model to use, not only for load calculations but also for power production. Design programs for turbine load calculations usually demand the mean wind speed at hub height as input. The wind profile is then adjusted according to the shear theory selected. In that case the differences will be similar to the plot shown in Figure $2.22 \mathrm{~b}$ : none at the hub and increasing slightly to the outer limits of the blades. The differences nearly cancel over the rotor disk.

Besides the difference in detail as demonstrated in this example, no real preference exists for either wind shear model.

\section{Turbulence}

When wind is measured in the field, a time varying wind speed can be found as shown in Figure 2.23. From this time series the statistical parameters can be calculated: the mean wind speed and the standard deviation.

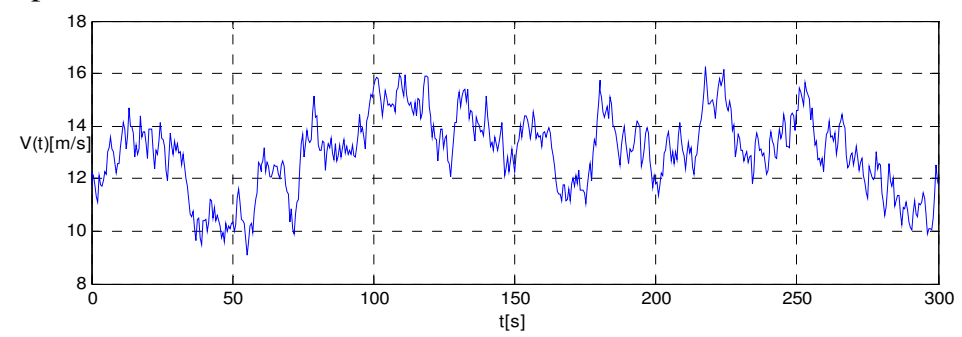

Figure 2.23 Time varying wind speed measurement

The turbulence intensity is defined as the standard deviation of the time varying wind speed divided by the mean wind speed, in percentage:

$$
I_{t}=\frac{\sigma}{V_{w}}[\%]
$$

The turbulence intensity is dependent on height and the roughness of the terrain. Rougher terrain and lower altitude give higher turbulence intensities. Design standards give descriptions of the turbulence intensity based on these roughness and altitude parameters. For design cases fixed turbulence levels can be selected for specific sites. Figure 2.24 shows the recommended turbulence intensities as a function of wind speed for different standards [20] [22] [23]. 


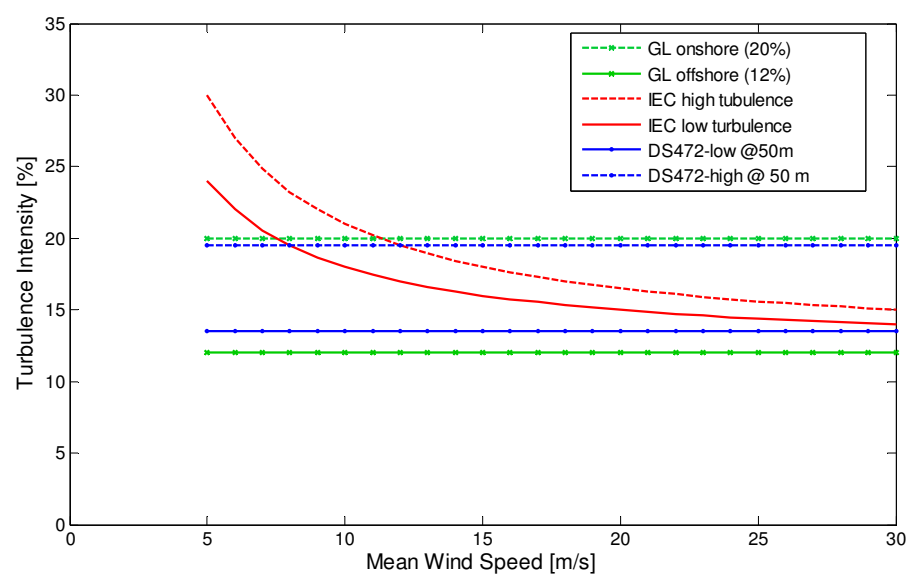

Figure 2.24 Turbulence intensity as a function of wind speed from different standards [20] [22] [23]

Next to the longitudinal turbulence (in the direction of the wind) also the lateral and vertical turbulence intensities are defined by the standards. Different descriptions are available ranging from isotropic turbulence $\left(I_{u}=I_{v}=I_{w}\right)$ to height dependent correlations.

The turbulence can also be represented in a spectral form. The basic shape was already shown in the right-hand side of the Van der Hoven spectrum (Figure 2.20). To enhance the readability of a turbulence spectrum, it is usually presented in log-log scale. Several models have been fitted to the turbulence spectrum. The von Kármán and Kaimal spectra are the most commonly used models [21] although turbulence spectra over sea generally contain even greater low frequency content. These models are based on the mean wind speed, the turbulence intensity and a length scale:

$$
\begin{gathered}
S_{\text {Kármán }}(f)=\frac{\sigma_{v}^{2} 4 L_{v} / V_{w}}{\left(1+70.8\left(f L_{v} / V_{w}\right)^{2}\right)^{5 / 6}} \\
S_{\text {Kaimal }}(f)=\frac{\sigma_{v}^{2} 4 L_{v} / V_{w}}{\left(1+6 f L_{v} / V_{w}\right)^{5 / 3}}
\end{gathered}
$$

with:

\begin{tabular}{l|l|l}
$\sigma_{v}$ & standard deviation of the wind speed & {$[\mathrm{m} / \mathrm{s}]$} \\
$L_{v}$ & integral length scale (model dependent) & {$[\mathrm{m}]$} \\
$V_{w}$ & mean wind speed & {$[\mathrm{m} / \mathrm{s}]$} \\
$f$ & frequency & {$[\mathrm{Hz}]$}
\end{tabular}


The precise definition of the length scales is again prescribed in the literature or in design standards. When these spectra are applied in calculation tools, generally these prescribed values can be selected. The underlying relationships between parameters are then fixed. No preference exists for either method.

\subsubsection{Extreme wind speeds and gusts}

Two basic extreme wind load cases can be defined: the extreme hourly mean wind speed with given return period (1, 50, 100 years) and the extreme incident wind speed within a given short time interval.

The extreme hourly mean wind speed is found by taking a large number of measured means and selecting the extremes above a chosen threshold. These extremes are then plotted on log-linear scale over the number of occurrences per time interval (month, year). By fitting a distribution curve through these measured values, an interpolation can be made of the expected maximum in periods beyond the measurement period. One of the most commonly used is the Gumbel distribution, as shown in Figure 2.25.

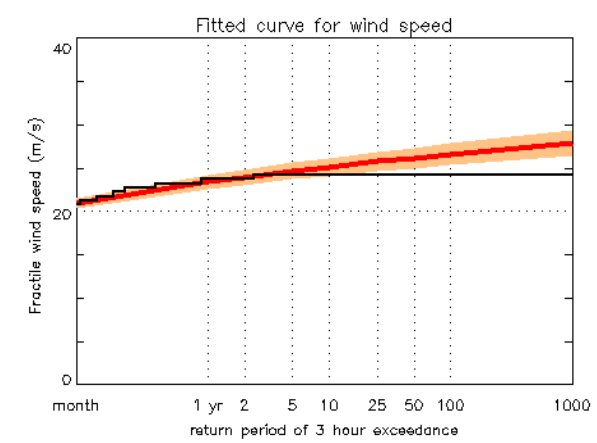

Figure 2.25 Gumbel fit (red line) for data recordings of mean wind speed (steps in black line) [24]

In onshore wind turbine design, the design standards give a prescribed extreme wind speed for different classes. Table 2.4 shows the values given by the IEC [22]. The classes are used as reference for the design of the turbine, they are not directly linked to specific sites. Offshore sites are classed as "S" (special), which are not defined and require site-specific analysis.

Table 2.4 Location classes according to IEC with yearly hourly mean wind speed ( $\left.V_{w, \text { ext lh }}\right)$ and extreme 10-minute mean wind speed $\left(V_{w, \text { ext }} 10 \mathrm{~min}\right)$ in $[\mathrm{m} / \mathrm{s}]$ at hub height

\begin{tabular}{l|c|c|c|c} 
Class & I & II & III & IV \\
\hline \hline$V_{w, \text { ext } 1 h}[\mathrm{~m} / \mathrm{s}]$ & 10 & 8.5 & 7.5 & 6.0 \\
$V_{w, \text { ext } 10 \min }[\mathrm{m} / \mathrm{s}]$ & 50 & 42.5 & 37.5 & 30
\end{tabular}


For offshore wind turbines the site-specific analysis can be based on extreme measured values and a Gumbel distribution, although the standard classes may give a fast first order estimate.

To determine extreme incident wind speed, or gust within a certain time interval, the tail of the turbulence spectrum can be used, but an approximate formulation was derived by Wieringa [25], which shows good agreement with theoretical results. The gust factor $G(t)$ is defined as:

$$
G(t)=1+0.42 I_{t} \ln \frac{3600}{t}
$$

The gust factor is used to convert the hourly mean wind speed to the gust wind speed for duration $t$ :

$$
V_{g u s t}(t)=G(t) \cdot V_{w}
$$

Figure 2.26 shows the gust factors for different durations for the turbulence intensity for onshore (20\%) and offshore (12\%) prescribed by Germanischer Lloyd [20].

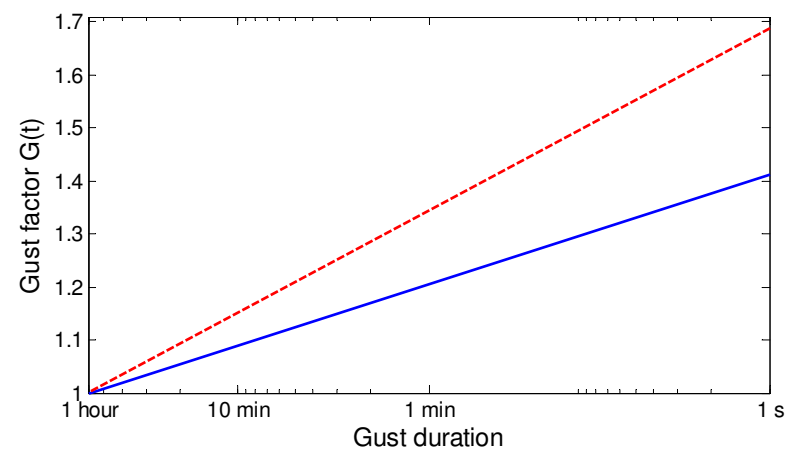

Figure 2.26 Gust factors for different durations and onshore (20\%) (dotted) and offshore (12\%) turbulence intensity as specified by GL [20]

\subsubsection{Long-term distribution of wind speed}

In addition to short-term load calculations, the wind climate at the site of an offshore wind farm needs to be known to assess the potential power production and for fatigue damage calculation. The first characteristic value in this respect is the yearly average wind speed. Figure 2.27 shows the average annual wind speed for the North Sea from [26]. 


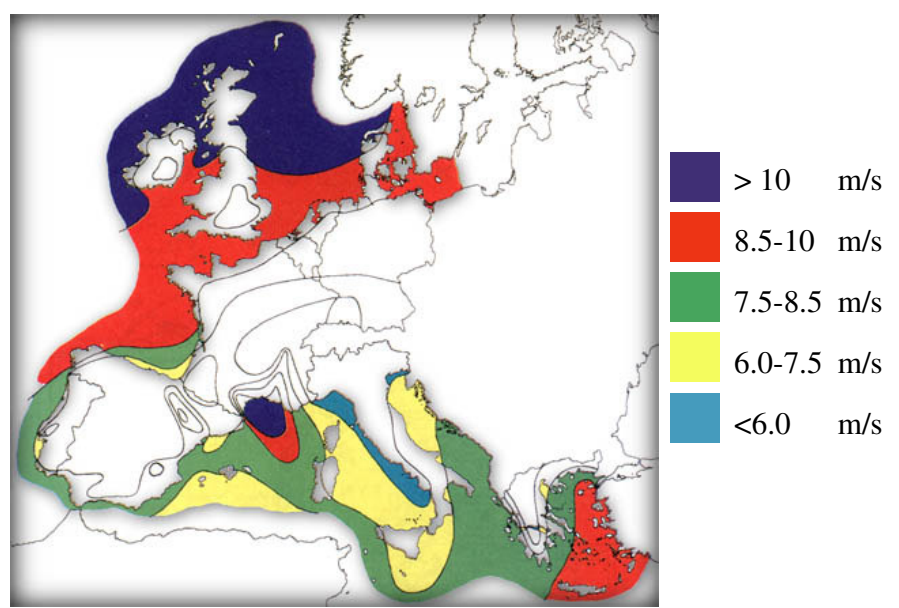

Figure 2.27 Yearly average wind speed at $100 \mathrm{~m}$ height for the European Seas [26]

A typical location will have values of $8 \mathrm{~m} / \mathrm{s}$ ranging up to $10 \mathrm{~m} / \mathrm{s}$ for the northern parts. For onshore sites the yearly average is significantly lower. Coastal sites may match the $8 \mathrm{~m} / \mathrm{s}$ but further inland the value drops rapidly to a typical value of $4 \mathrm{~m} / \mathrm{s}$ for wooded southern Germany. Local landscape features have an effect on the average yearly wind speed. On top of hills and mountains the value may be significantly higher; an island can reduce it.

The annual wind speed distribution is very site-specific and because yearly averages are also variable, long-term measurements are preferable. Figure 2.28 shows the yearly distributions of wind speeds for Horns Rev for various wind directions [27].

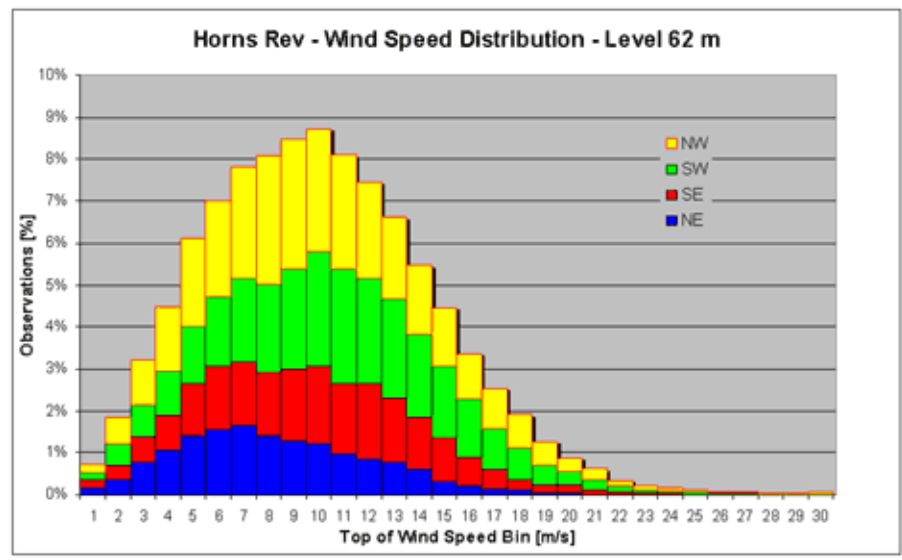

Figure 2.28 Annual wind speed distribution for various wind directions measured at Horns Rev at $62 \mathrm{~m}$ height [27] 
A common way to describe the annual wind speed distribution is by use of the Weibull distribution. The curve represents a fit of the probability density per wind speed at a given location as shown in Figure 2.28 for Horns Rev. It is based on two parameters: a scale parameter $c_{w}$, related to the annual mean wind $V_{w, y e a r}$ speed and a shape parameter $k_{w}$ describing the variability about the mean. Equation (2.22) represents the Weibull probability density function [28]:

$$
f\left(V_{w}\right)=\frac{k_{w}}{c_{w}}\left(\frac{V_{w}}{c_{w}}\right)^{k_{w}-1} e^{-\left(\frac{V_{w}}{c_{w}}\right)^{k_{w}}}
$$

From this expression the yearly mean can be reproduced analytically. A faster method is the empirical formulation by Lysen [66]:

$$
V_{w, \text { year }}=c_{w}\left(0.568+0.433 / k_{w}\right)^{\frac{1}{k_{w}}}
$$

Typical values for $k_{w}$ are: 1.75 for inland areas, 2.0 on the coast and 2.2 offshore. A higher $k_{w}$ value means that the peak becomes lower and the tails flatter, meaning that the distribution contains less extreme high or low values. For offshore locations this is true because diurnal patterns (heating of the surface in the daytime, cooling at night) is much less significant. Figure 2.29 shows the Weibull distributions for typical onshore, coastal and offshore conditions.

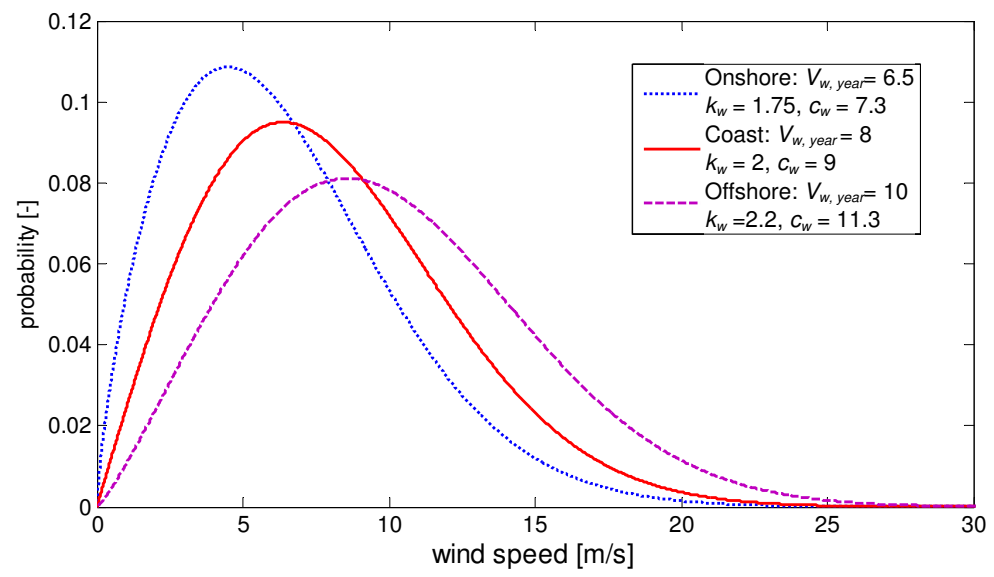

Figure 2.29 Weibull annual wind distributions for typical onshore, coast and offshore conditions 


\subsection{Description of the turbine}

\subsubsection{Introduction}

Wind turbines come in many different types and shapes. This thesis only deals with the most commonly used type in present-day wind energy production: the upwind, 3 bladed wind turbine.

\subsubsection{Modern turbine characteristics}

First and most important, a wind turbine produces electricity. The turbine does so by slowing down the flow of air through its rotor plane. The air flow is transformed to kinetic energy in the rotating blades driving the generator, which in turn converts the kinetic energy to electric power. The components facilitating these conversions of energy are housed in the nacelle as shown in Figure 2.30

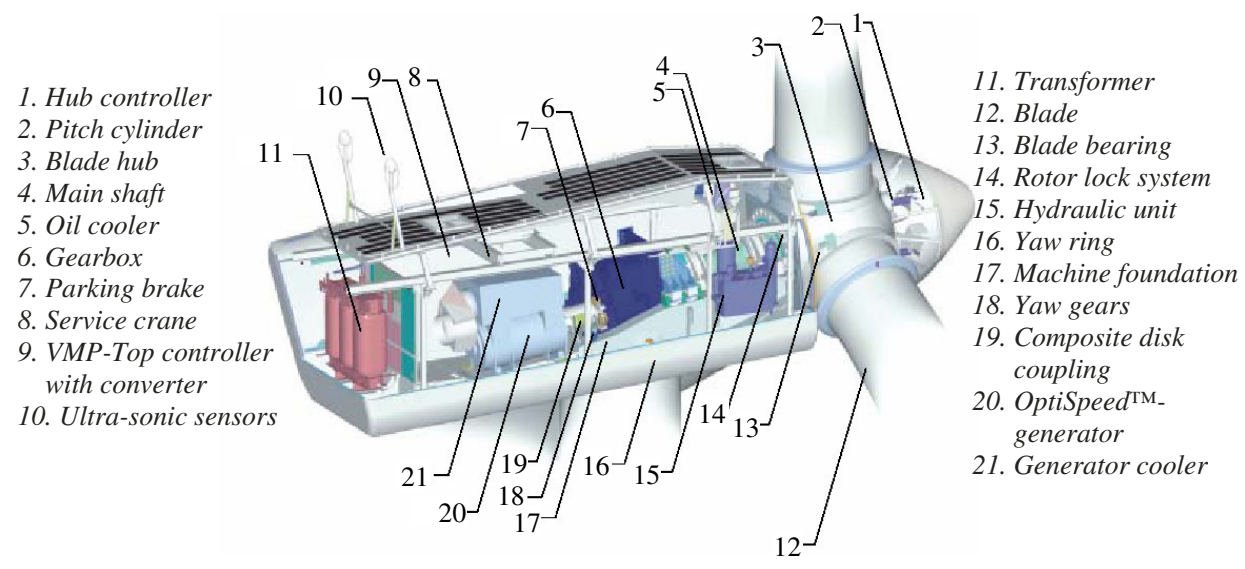

Figure 2.30 Components inside the nacelle of the Vestas V80 turbine used at Horns Rev [29]

To calculate the loads on the turbine, the blade element momentum theory is used. The momentum theory assumes a stream tube as shown in Figure 2.31. The turbine can be seen as an actuator disk, slowing the flow of air, creating a wake with reduced air flow velocity. The load that the actuator disk exerts on the flow to achieve this is the axial load $F_{a x}$. 


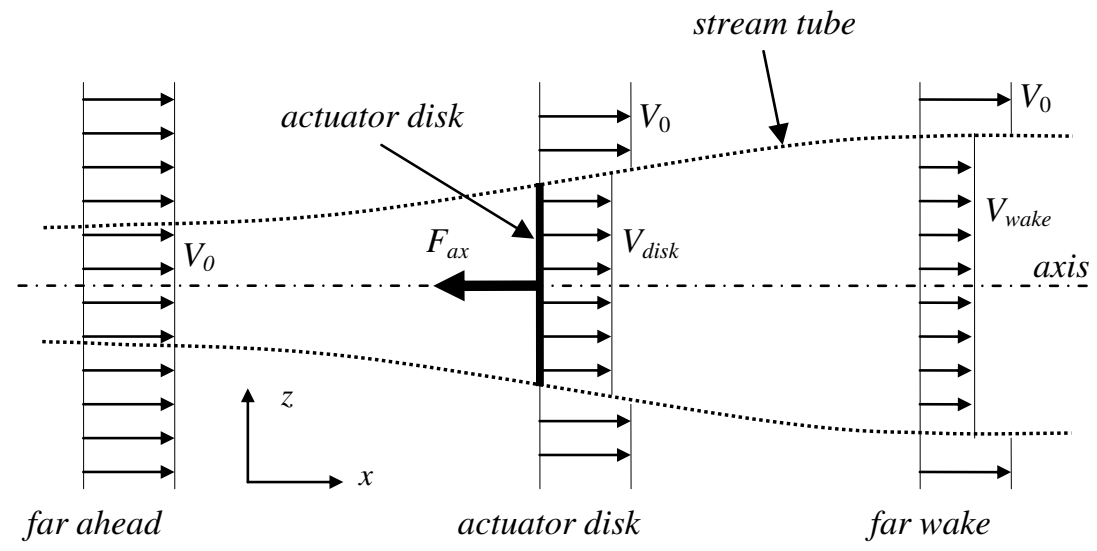

Figure 2.31 Actuator disk with stream tube

By defining the induction factor $a$, equation (2.24), the axial force of the actuator disk on the flow of air can be found using the momentum theory assuming incompressible, homogeneous and horizontal flow:

$$
a=\frac{V_{0}-V_{d i s k}}{V_{0}}
$$

Using the Bernoulli to calculate the resulting load on the actuator disk, we find [21]:

$$
F_{a x}=\frac{1}{2} A_{d i s k} \rho_{a i r} V_{0}^{2} \cdot 4 a(1-a)
$$

with:

\begin{tabular}{l|l|l}
\hline$a$ & induction factor & {$[-]$} \\
$V_{0}$ & undisturbed wind velocity & {$[\mathrm{m} / \mathrm{s}]$} \\
$V_{d i s k}$ & wind velocity at the actuator disk & {$[\mathrm{m} / \mathrm{s}]$} \\
$A_{d i s k}$ & area of the rotor disk & {$\left[\mathrm{m}^{2}\right]$}
\end{tabular}

Because neither the axial load nor the induction factor is known, a second theory is used to solve the equation: the blade element theory. The blade is thought to consist of blade elements each with their own aerodynamic features. The blades are shaped according to a series of airfoil profiles to create the desired aerodynamic performance. The airfoils near the root have a large thickness to accommodate structural elements to transfer the blade loads to the rotor axis. Near the tip, the airfoils are more optimised to performance only. The airfoil variation is shown in Figure 2.32. The airfoils originate 
from aero-elastic design programs and are validated in test projects that describe their shape and aerodynamic performance as tested in wind tunnels.

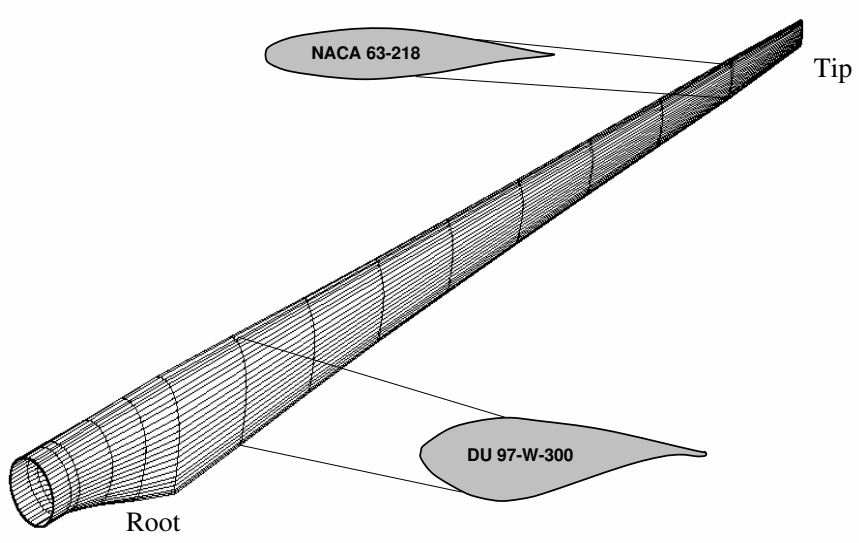

Figure 2.32 Rotor blade with thick airfoils at the root, thin airfoils at the tip

The wind load on a structure can be calculated with an equation similar to that for the calculation of hydrodynamic loads. The density of the air and the air flow velocity are used together with an aerodynamic coefficient that is dependent on the surface roughness, the shape of the structure and the air flow velocity. For a single section of a tubular tower its value is typically $C_{\text {aero }}=0.7$, which is the drag coefficient. The wind load on this section is given by:

$$
F_{\text {aero }}=\frac{1}{2} C_{\text {aero }} \rho_{\text {air }} A V_{\text {section }}^{2}
$$

with:

\begin{tabular}{l|l|c}
\hline$F_{\text {aero }}$ & wind load & {$[\mathrm{N}]$} \\
$C_{\text {aero }}$ & aerodynamic coefficient (shape, surface dependent) & {$[-]$} \\
$\rho_{\text {air }}$ & density of air $(1.225)$ & {$\left[\mathrm{kg} / \mathrm{m}^{3}\right]$} \\
$A$ & exposed area of the section & {$[\mathrm{m}]$} \\
$V_{\text {section }}$ & wind velocity at the centre of the section & {$[\mathrm{m} / \mathrm{s}]$}
\end{tabular}

Airfoils experience not only a drag force, but also a lift force, which is calculated in the same manner. The lift and drag coefficients for airfoils are dependent on the angle of attack as shown in Figure 2.33. 


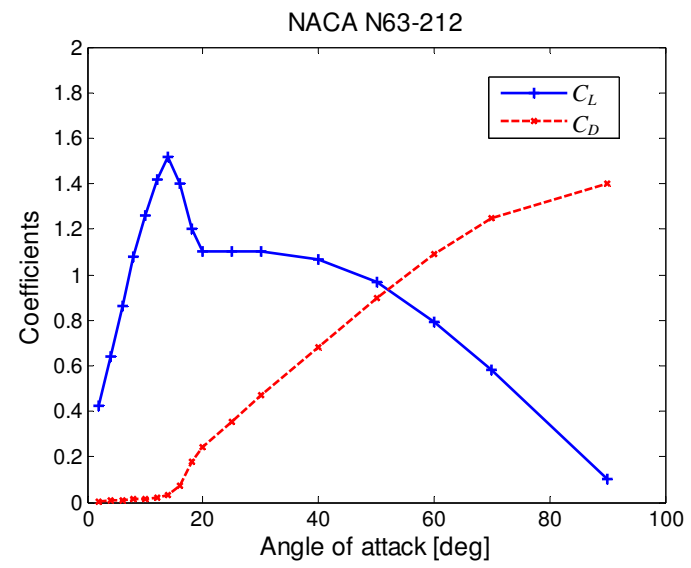

Figure 2.33 Lift coefficient $\left(C_{L}\right)$ and drag coefficient $\left(C_{D}\right)$ curve per angle of attack $\alpha$ for the NACA N63-212 airfoil

To calculate the aerodynamic loads on the blade, the blade is thought to be made of blade elements, all with their own airfoil characteristics. These elements are thought to be infinitely long, so no boundary effects are taken into account and there is no aerodynamic interaction between the elements. The loads on the blade elements are generated by lift and drag, which are induced by the wind speed and the rotation speed, combined in the relative wind speed over the blade:

$$
\begin{gathered}
V_{r e l}=\sqrt{V_{d i s k}^{2}+V_{r o t}^{2}} \\
V_{d i s k}=V_{0}(1-a) \text { and } V_{r o t}=\Omega \cdot r
\end{gathered}
$$

with:

\begin{tabular}{l|l|l}
\hline$V_{\text {rel }}$ & relative wind speed at a blade section & {$[\mathrm{m} / \mathrm{s}]$} \\
$V_{\text {disk }}$ & wind velocity at airfoil & {$[\mathrm{m} / \mathrm{s}]$} \\
$V_{\text {rot }}$ & linear rotation speed at a blade section & {$[\mathrm{m} / \mathrm{s}]$} \\
$\Omega$ & angular rotation speed & {$[\mathrm{rad} / \mathrm{s}]$} \\
$r$ & distance of blade element to axis of rotation & {$[\mathrm{m}]$}
\end{tabular}

Due to this relative wind velocity, lift and drag loads are exerted on the blade element as shown in Figure 2.34. Note that the drag load is not drawn on the same scale as the lift load. Would that be the case, the drag load arrow $\left(F_{D}\right)$ would be shorter than the size of its tip, making it invisible in the plot. The lift and drag loads are calculated with equations (2.28). 

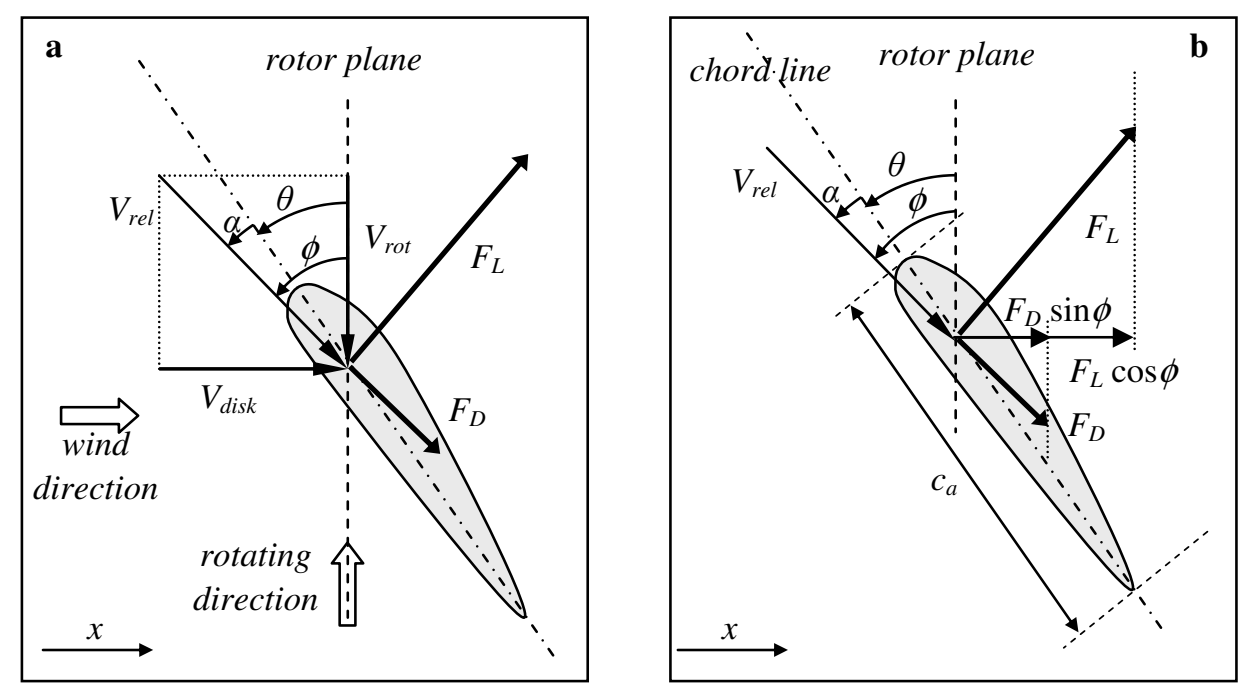

Figure 2.34 a) Lift and drag load on the blade element b) Resulting loads in the $x$ direction

$$
F_{L}=\frac{1}{2} C_{L}(\alpha) \rho_{a i r} V_{r e l}^{2} c_{a} \Delta r \quad F_{D}=\frac{1}{2} C_{D}(\alpha) \rho_{\text {air }} V_{r e l}^{2} c_{a} \Delta r
$$

with:

\begin{tabular}{l|l|l}
\hline$F_{L}$ & aerodynamic lift & {$[\mathrm{N}]$} \\
$F_{D}$ & aerodynamic drag & {$[\mathrm{N}]$} \\
$C_{L}(\alpha)$ & aerodynamic lift coefficient & {$[-]$} \\
$C_{D}(\alpha)$ & aerodynamic drag coefficient & {$[-]$} \\
$\rho_{\text {air }}$ & mass density of air & {$\left[\mathrm{kg} / \mathrm{m}^{3}\right]$} \\
$c_{a}$ & airfoil chord length & {$[\mathrm{m}]$} \\
$\Delta r$ & radial length of blade element & {$[\mathrm{m}]$} \\
$\alpha$ & angle of attack & {$[\mathrm{deg}]$} \\
$\theta$ & pitch angle & {$[\mathrm{deg}]$} \\
$\phi$ & angle of inflow & {$[\mathrm{deg}]$}
\end{tabular}

The load in $x$ direction per blade element is:

$$
F_{x}=F_{L} \cos \phi+F_{D} \sin \phi
$$

Making the total axial load:

$$
F_{a x}=N_{b} \sum_{r=\text { root }}^{r=t i p} F_{x, r}
$$


When the axial load is solved iteratively by using the blade element theory (2.30) and the momentum theory (2.25), the aerodynamic loads on the structure are known. From this, the power production can be calculated:

$$
P=F_{a x} V_{d i s k}=2 \rho_{\text {air }} A_{\text {disk }} V_{0}^{3} a(1-a)^{2}
$$

By defining the power coefficient $C_{p}$ as the power $P$ divided by the total power in the air flow through the rotor plane, equation (2.32) is found:

$$
C_{P}=\frac{P}{\frac{1}{2} \rho_{\text {air }} V_{0}^{3} A_{\text {disk }}}=4 a(1-a)^{2}
$$

The maximum of the power coefficient can be determined to be $C_{P}=16 / 27=0.593$ at $a=1 / 3$ which is known as the Lanchester-Betz limit [30].

The blade element momentum theory is far more refined than basics described here, incorporating, among others, tip and root effects. These effects are described well in [21] and are incorporated in Bladed, which has been used for turbine modelling in this thesis.

\subsubsection{Managing power capture}

Although the theoretical maximum power extraction from the wind is limited at the Betz limit, several parameters influence the actual power capture. If we take a blade configuration optimised to aerodynamic power capture capabilities through its airfoils and twist along the blade to make the angle of attack optimal per airfoil, the power will still be dependent on the relation between rotor speed and wind speed, combined in the relative wind speed $V_{\text {rel }}$ in equation (2.27). We define $\lambda$ as the tip speed ratio: the ratio between the speed of the blade tip and the wind speed:

$$
\lambda=\frac{V_{t i p}}{V_{0}}=\frac{\Omega R}{V_{0}}
$$

The relation between the tip speed ratio and the power coefficient is plotted in Figure 2.35. The curve shows a peak, for this particular blade, at $\lambda=8$. 


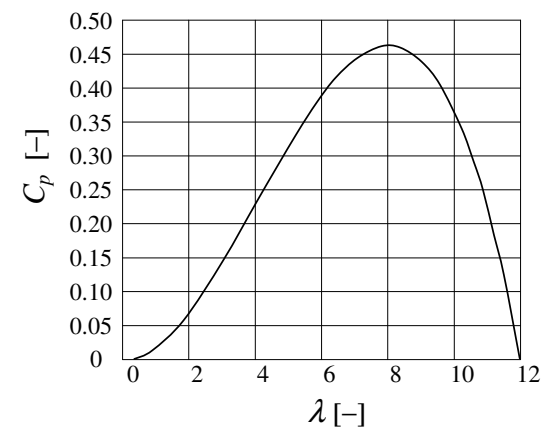

Figure 2.35 Typical $C_{P^{-}} \lambda$ curve

To capture maximum power at every wind speed, the rotation speed should be changed to keep the $C_{P}-\lambda$ curve at its maximum. Figure 2.36 shows the power capture of a variable speed turbine tuning its rotation speed to maximum power. Because the generator is connected to the electricity grid, the grid demands the power output to be of constant frequency. Because of advances in power electronics, the rotation speed and the grid frequency can nowadays be fully uncoupled without loss of power quality. But in the recent past, this option was not always available. Turbines were at that time fitted with constant speed generators. The power capture could therefore only be optimal at one wind speed, which is also shown in Figure 2.36.

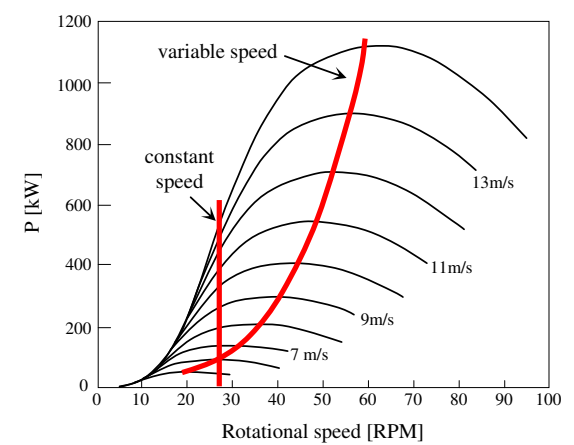

Figure 2.36 Power output as function of rotational speed for different wind speed classes

In Figure 2.36, the power capture of the variable speed turbine continuously increases with higher wind speeds. For actual turbines, the power capture is cut-off at a certain point. The maximum of the power capture is limited by the generator size. The optimum rotor-generator configuration is the result of economic optimisation. The wind speed at which the generator reaches maximum power is called the rated wind speed, $V_{\text {rated }}$. To limit the power capture to match the generator, two basic options exist. 
When the blade is rigidly connected to the hub, the angle of attack and the lift coefficient will increase with the wind speed to a maximum value, as shown in Figure 2.33 and Figure 2.34. When the wind speed increases further, the lift coefficient will decrease: the airflow over the blade becomes detached, the blade stalls. This way the amount of energy extracted from the wind is limited. This is the concept of the stallregulated turbine. A variation of this concept is active stall: the blade is pitched into the wind to induce stall at the desired moment.

The other option to control the power output above rated wind speed is to pitch the blade tail away from the wind making the angle of attack smaller, corresponding with the lower lift coefficients on the left-hand side of Figure 2.33.

The relation between wind speed and power production is now fully described in theory. A wind turbine needs a certain minimum wind speed to start operation, typically $3 \mathrm{~m} / \mathrm{s}$. This wind speed is called the cut-in speed, $V_{\text {cut-in }}$. From this wind speed, the turbine power follows the power curve until the rated power is reached, $V_{\text {rated }}$. Above rated wind speed the turbine remains producing rated power. Finally, for very high wind speeds, the turbine is shut down. This happens at $V_{\text {cut-out }}$, the cut-out wind speed. For present-day turbines $V_{\text {cut-out }}=25 \mathrm{~m} / \mathrm{s}$ typically, although some turbines continue to $V_{\text {cut-out }}=36 \mathrm{~m} / \mathrm{s}$. This value is also the result of cost-component optimisation. Figure 2.37 shows the power (or $P-V$ ) curve for the Vestas V80 turbine [29].

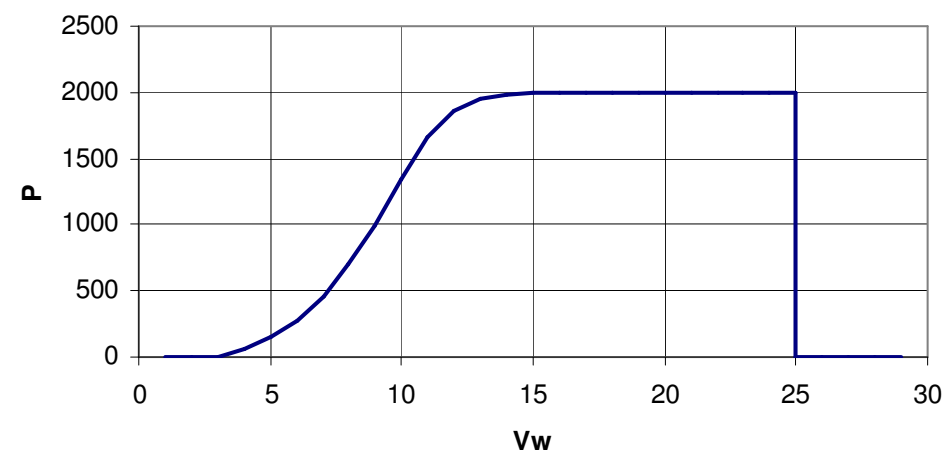

Figure 2.37 Power curve for Vestas V80 turbine [29]

\subsubsection{Annual power production}

The power production of turbines will eventually always be the bottom line of any offshore wind farm project. With long-term wind speed measurements for a specific site, an optimum turbine configuration and a minimum of downtime, detailed yearly production calculations can be made. A very crude rule of thumb to take all effects of lower wind speeds and downtime into account is to estimate the total production to be $1 / 3$ of the rated turbine output [31]. This would mean that a $160 \mathrm{MW}$ rated output offshore wind farm would produce $160 * 1 / 3 * 8760=467200 \mathrm{MWh} /$ year. An average 
European household uses $4000 \mathrm{kWh} /$ year [27], which means the offshore wind farm is able to supply some 150000 homes throughout a year. For Horns Rev in Denmark, this means the $160 \mathrm{MW}$ station is providing $2 \%$ of the total Danish power consumption.

\subsubsection{The turbine in turbulent flow}

When a turbine is in operation, its rotor rotates through a three-dimensional turbulence field. This field can be described by the correlated turbulence effects described in section 2.5. A graphic representation of such a turbulence field is shown in Figure 2.38. This turbulence field was modelled in Bladed for the Blyth turbine, covering an area of 100 by $100 \mathrm{~m}$ at a wind speed of $10 \mathrm{~m} / \mathrm{s}$ with $12 \%$ turbulence intensity. Areas of higher and lower wind speeds are clearly distinguishable.

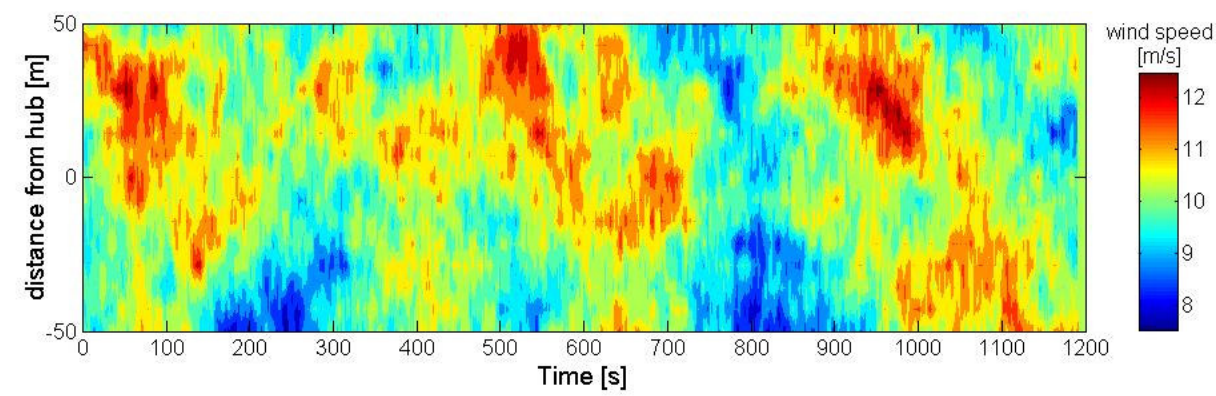

Figure 2.38 Turbulent eddies in a wind field

When a rotating blade passes through an eddy, it experiences a short period of higher or lower wind speed. Figure 2.39 zooms in on such an eddy occurring between $500 \mathrm{~s}$ and 550s in Figure 2.38. Because the size of the eddy is such that a blade will pass through the eddy several times, the turbine structure will experience a load peak at the rotation frequency of the rotor, called 1P; upper figure. This eddy slicing will create not only a load peak at the frequency of $1 \mathrm{P}$ but also at the frequency of all the blades passing: $N_{b} \mathrm{P}=3 \mathrm{P}$ for a three-bladed turbine as shown in the lower figure. 

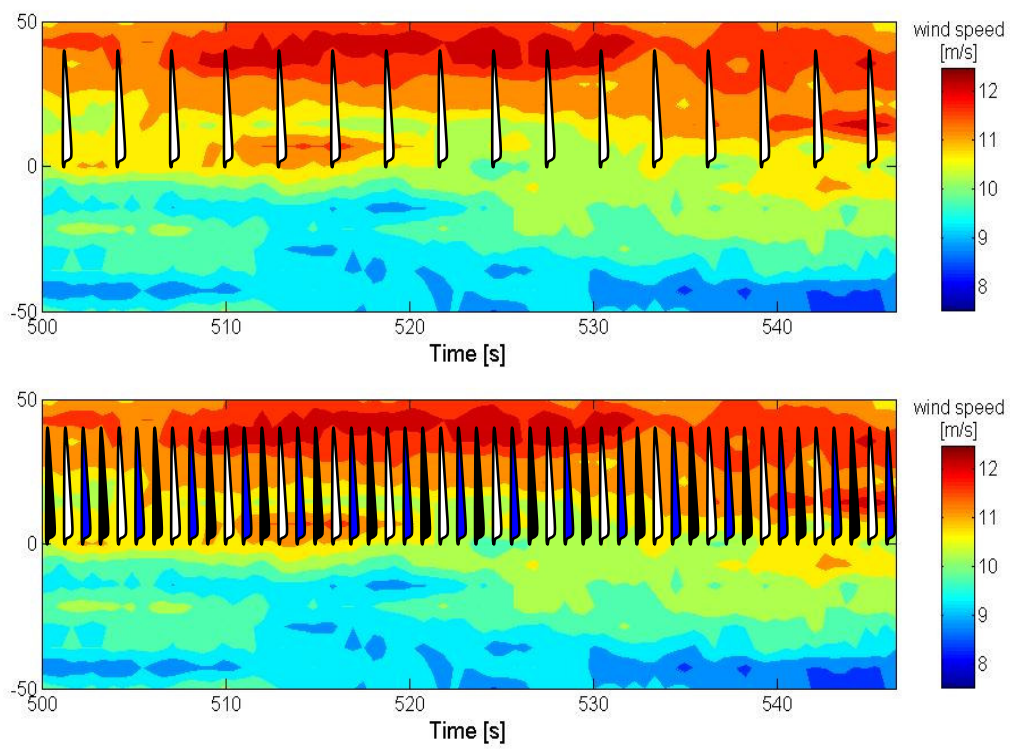

Figure 2.39 Single blade passing through turbulent eddy (above) sampling 1P frequency All three blades (black, white, blue) passing through the eddy at $3 \mathrm{P}$ frequency

To take this effect into account within the turbulence spectrum, the stationary turbulence spectrum, as described in section 2.5 , can be transformed into a rotationally sampled spectrum. An example of a stationary and a rotationally sampled velocity spectrum is shown in Figure 2.40.

a

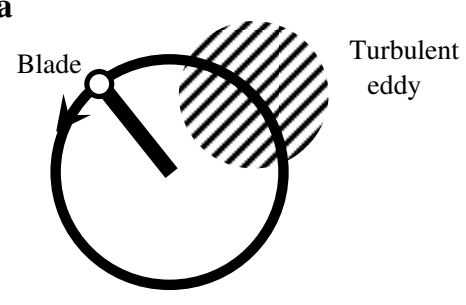

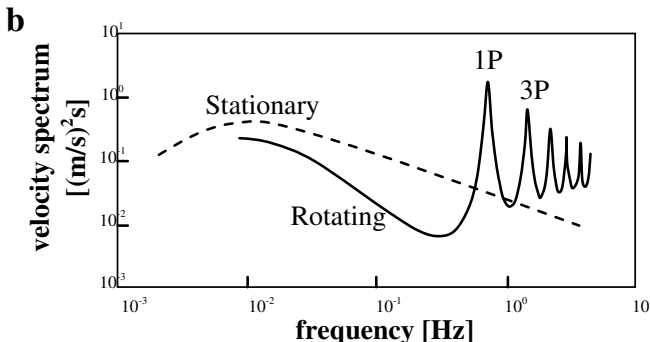

Figure 2.40 a) Blade passing through a turbulent eddy

b) Stationary and rotationally sampled turbulence spectrum 


\subsection{Dynamics of offshore wind turbines}

\subsubsection{The basics of dynamics}

The importance of proper modelling of the structural dynamics can be most conveniently illustrated by considering a single degree of freedom mass-spring-damper system as shown in Figure 2.41. A complete offshore wind turbine system can be thought of as being constructed of a number of coupled multi degree-of-freedom massspring-damper systems [32].

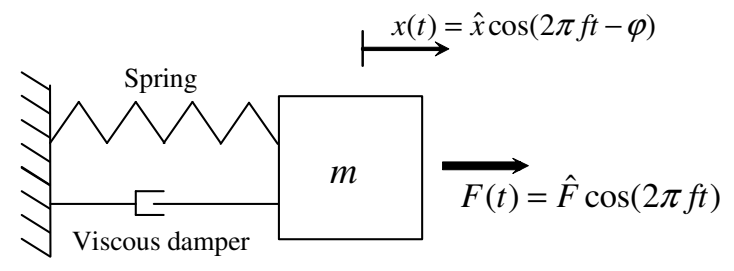

Figure 2.41 Single degree of freedom mass-spring-damper system

When a harmonic excitation $F(t)$ is applied to the mass, the magnitude and phase of the resulting displacement $x$ strongly depend on the frequency of excitation $f$. Three steady state response regions can be distinguished as shown in Figure 2.42:

a) Quasi-static

b) Resonance

c) Inertia dominated.
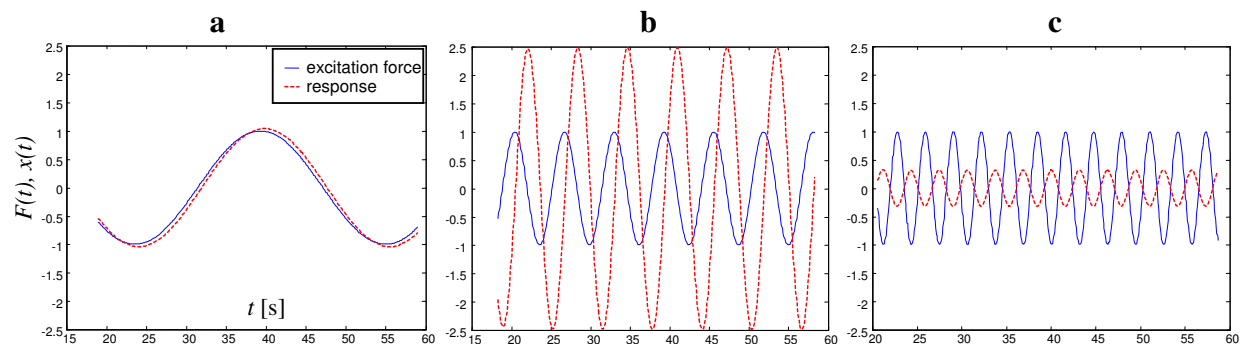

Figure 2.42 a) Quasi-static b) resonant and c) inertia dominated response Solid blue line: excitation, dashed red line: displacement

For frequencies of excitation well below the natural frequency of the system, the response is quasi-static, as illustrated in Figure 2.42a: the displacement of the mass follows the time varying force almost instantaneously, as if it was excited by a static load. Figure $2.42 \mathrm{~b}$ shows a typical response for frequencies of excitation within a 
narrow region around the system's natural frequency. In this region the spring force and the inertia force (almost) cancel, producing a response that is a number of times larger than it would be statically. The resulting amplitude is governed by the damping present in the system. For frequencies of excitation well above the natural frequency, the mass cannot "follow" the excitation any more. Consequently, the response level is low and almost in counter-phase, as illustrated in Figure 2.42c. In this case the inertia of the system dominates the response.

Figure 2.42 illustrates the general fact that, in steady state, a sinusoidal input applied to a linear system generates a sinusoidal output at the same frequency, which differs in magnitude and phase.

The magnitude and phase modifying property of linear systems can be summarized by a plot of the dynamic amplification factor (DAF) and the related phase lag. The DAF depicts the ratio between the dynamic response magnitude and the static response magnitude due to the same magnitude of loading. Figure 2.43 shows the DAF and phase lag plot of the single degree of freedom system depicted in Figure 2.41.
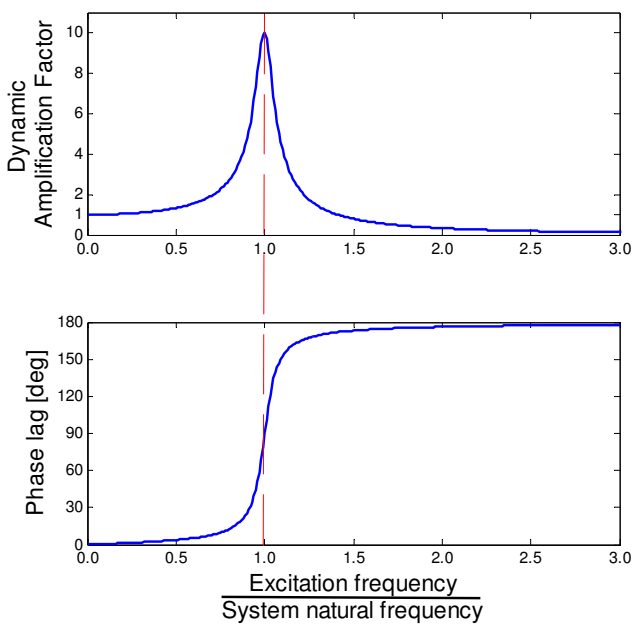

Figure 2.43 Upper figure: dynamic amplification factor per normalised frequency lower figure: phase lag versus normalised frequency

The peak in Figure 2.43 corresponds to the system's natural frequency. The height of the peak is determined by damping. Therefore any resonant problem can be counteracted with adequate damping. In dynamics, the frequency of excitation is at least as important as its magnitude. Resonant behaviour can cause severe load cases, even failure, but is most feared because of potential fatigue problems. For structures where 
dynamics are expected to be a problem, detailed knowledge of the expected frequencies of excitation and the natural frequencies of the structure or part of the structure is vital.

The DAF is commonly used in calculations in the preliminary design phase to account for the effect of dynamic response compared to static response (thereby neglecting the phase information). In general, the DAFs are derived from time-domain simulations similar to the ones shown in Figure 2.43.

The important conclusion that can be drawn from this review is that the response of a wind turbine system subjected to time-varying loads needs to be carefully assessed.

\subsubsection{Soft and stiff wind turbine systems}

\section{Excitation}

To translate the basic model of the previous section to a wind turbine system, first the excitation frequencies are examined first. The most visible source of excitation in a wind turbine system is the rotor. As shown in section 2.6.5, the rotor samples the turbulent eddies in the wind field creating peaks in excitation at frequencies of $1 \mathrm{P}$ and 3P for a three bladed rotor.

These two frequencies are plotted in a graph as shown in Figure 2.44. The horizontal axis represents the frequency $[\mathrm{Hz}]$ and the vertical axis represents an arbitrary response without values. Though higher order excitations do occur, here only $1 \mathrm{P}$ and $3 \mathrm{P}$ are considered as these are the primary excitations. To avoid resonance, the structure should be designed such that its first natural frequency does not coincide with either $1 \mathrm{P}$ or $3 \mathrm{P}$ excitation. This leaves three possible intervals. A very stiff structure, with its first natural frequency above $3 \mathrm{P}$ is called a stiff-stiff structure; if the first natural frequency falls between $1 \mathrm{P}$ and $3 \mathrm{P}$, the structure is said to be soft-stiff while a very soft structure with its first natural frequency below $1 \mathrm{P}$ is called a soft-soft structure.

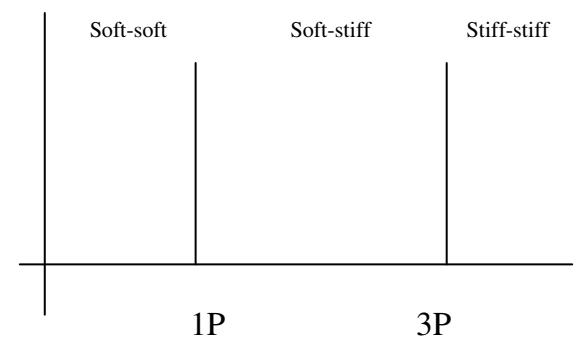

Figure 2.44 Soft to stiff frequency intervals of a three bladed, constant rotational speed wind turbine

The support structure

A flexible wind turbine can be modelled as a flagpole with top mass $m_{t o p}$, as depicted in Figure 2.45. This model resembles the model of the mass-spring-damper system in 
Figure 2.41. The bending flexibility of the tower represents the spring stiffness; the damping is given in the form of a damping coefficient.

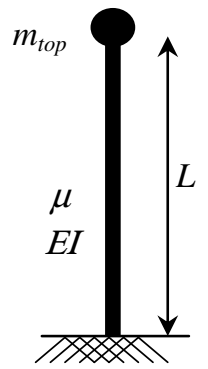

Figure 2.45 Structural model of a flexible wind turbine system

For this model consisting of a uniform beam with a top mass and a fixed base, the following approximation for the calculation of the first natural frequency is valid [33]:

$$
f_{\text {nat }}^{2} \cong \frac{3.04}{4 \pi^{2}} \frac{E I}{\left(m_{\text {top }}+0.227 \mu L\right) L^{3}}
$$

with:

\begin{tabular}{l|l|l}
\hline$f_{\text {nat }}$ & first natural frequency & {$[\mathrm{Hz}]$} \\
$m_{\text {top }}$ & top mass & {$[\mathrm{kg}]$} \\
$\mu$ & tower mass per meter & {$[\mathrm{kg} / \mathrm{m}]$} \\
$L$ & tower height & {$[\mathrm{m}]$} \\
$E I$ & tower bending stiffness & {$\left[\mathrm{Nm}^{2}\right]$}
\end{tabular}

Using the parameters in equation (2.35):

$$
I \cong \frac{1}{8} \pi D_{a v}{ }^{3} t_{w} \text { and } \mu=\rho_{\text {steel }} \pi D_{a v} t_{w} \text { and } a=\frac{m_{\text {top }}}{\rho_{\text {steel }} \pi D_{a v} t_{w} L}
$$

equation (2.34) can be re-written as:

$$
f_{\text {nat }} \cong \frac{D_{a v}}{L^{2}} \sqrt{\frac{E}{104(a+0.227) \rho_{\text {steel }}}}
$$

where:

\begin{tabular}{l|l|l}
$t_{w}$ & tower wall thickness & {$[\mathrm{m}]$} \\
$D_{a v}$ & tower average diameter $=D-t_{w}$ & {$[\mathrm{~m}]$} \\
$\rho_{\text {steel }}$ & density of steel $(7850)$ & {$\left[\mathrm{kg} / \mathrm{m}^{3}\right]$}
\end{tabular}


By way of example, equation (2.36) is applied to the Opti-OWECS design [5]. This design consists of a 2 bladed constant speed turbine. The rotational frequency $1 \mathrm{P}$ is 0.3 $\mathrm{Hz}$, the blade passing frequency $2 \mathrm{P}$ is $0.6 \mathrm{~Hz}$. A soft-soft structure would then have (for example) a first natural frequency of $0.25 \mathrm{~Hz}$, soft-stiff $0.5 \mathrm{~Hz}$ and stiff-stiff $1 \mathrm{~Hz}$. The wall thickness is taken to be constant over the entire height: $75 \mathrm{~mm}$. The system has a top mass of $130000 \mathrm{~kg}$. Equation (2.36) is applied to determine the diameter $D$ corresponding to the specified natural frequencies. The results are listed in table 2.5.

Table 2.5 Required diameters per frequency

\begin{tabular}{c|c|c} 
Type & $f_{\text {nat }}$ & Diameter \\
\hline \hline Soft-soft & $0.25 \mathrm{~Hz}$ & $2.4 \mathrm{~m}$ \\
Soft-stiff & $0.5 \mathrm{~Hz}$ & $4.2 \mathrm{~m}$ \\
Stiff-stiff & $1.0 \mathrm{~Hz}$ & $7.4 \mathrm{~m}$
\end{tabular}

As the cost of procurement and handling of large tubular towers is mainly influenced by the diameter, from an investment point of view the selection of the "softest" structure will be best.

These calculations are for demonstration purposes only, since an actual offshore wind turbine support structure will be dimensioned for many more influences. For instance, the soil properties will always be more flexible than the assumed fixed connection of the model in Figure 2.45. This flexibility will result in a lower overall natural frequency, which may need to be compensated by increasing the diameter.

\subsubsection{Design options for support structure dynamics}

\section{Variable speed}

As described in section 2.6, variable speed turbines are gaining market share from constant speed turbines. They offer higher energy capture and lower dynamic excitation. For example, the Vestas $2 \mathrm{MW}$ turbines have a rotational speed ranging from 10.5 to 24.5 RPM [29]. This means that the interval for a soft-stiff design is correspondingly narrower, as shown in Figure 2.46.

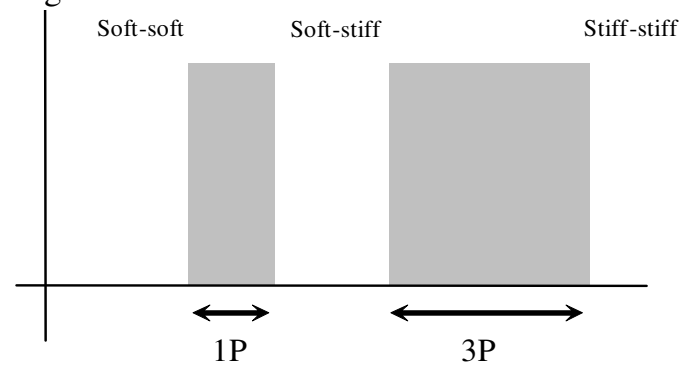

Figure 2.46 Frequency intervals for a variable speed turbine system 


\section{Larger turbines}

The trend to create larger turbines is still strong. This means that rotor blades become longer and tower top masses (generator) larger. The increase in rotor diameter has a direct effect on the soft to stiff approach. As shown in section 2.6.3, the power performance of a turbine can be represented as a function of tip speed ratio as shown in Figure 2.35. The tip speed ratio was defined in equation (2.33) and can be expressed as:

$$
\lambda=\frac{V_{\text {tip }}}{V_{w}}=\frac{\Omega R}{V_{w}}=\frac{f_{1 P} \pi D_{\text {rotor }}}{V_{w}}
$$

The corresponding 1P rotational frequency is given by:

$$
f_{1 P}=\frac{\lambda V_{w}}{\pi D_{\text {rotor }}}
$$

This means that for a fixed (optimal) tip speed ratio the rotational frequency will decrease when the diameter increases. The results of equation (2.38) for a wind speed of $V_{w}=11.4 \mathrm{~m} / \mathrm{s}, \lambda=8$ and rotor diameters of $80 \mathrm{~m}, 100 \mathrm{~m}$ and $120 \mathrm{~m}$, respectively, are plotted in Figure 2.47.

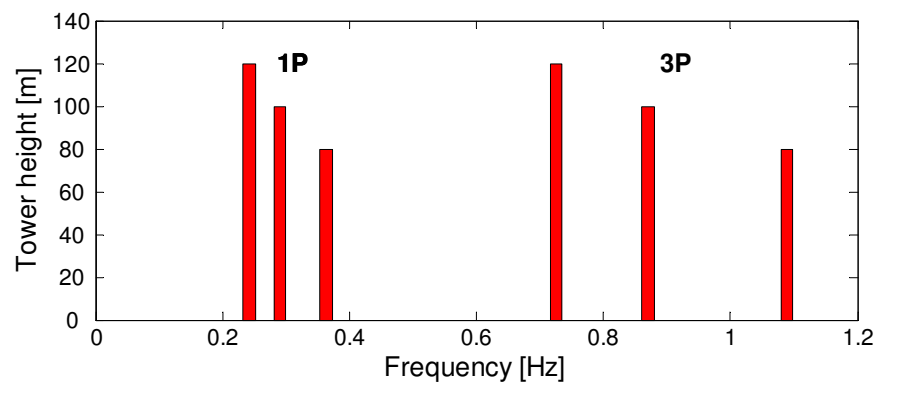

Figure 2.47 $1 \mathrm{P}$ and $3 \mathrm{P}$ frequencies for 80,100 and $120 \mathrm{~m}$ diameter rotors operating at constant rotational speed

The increase in rotor diameter also requires a higher hub height. From equation (2.36) the natural frequency is seen to be inversely proportional to the tower height $L$ squared. This means a large decrease of natural frequency with increasing height.

\section{Wave excitation}

For offshore wind turbine systems an additional source of excitation is present in the waves. Wave frequencies are generally lower than the rotational frequency of the rotor. Because waves come in various periods they span a wide range in the frequency band. 
Figure 2.48 shows the range of mean wave frequencies occurring at the NL1 location, the location of the previously mentioned Opti-OWECS turbine near the Dutch coast [5]. The histogram shows the relative occurrence per year of the wave frequencies. From Figure 2.48, it is clear that when the offshore wind turbine system is designed with a natural frequency below the rotational frequency to avoid resonance, it will come into the area where resonance due to wave excitation will become important.

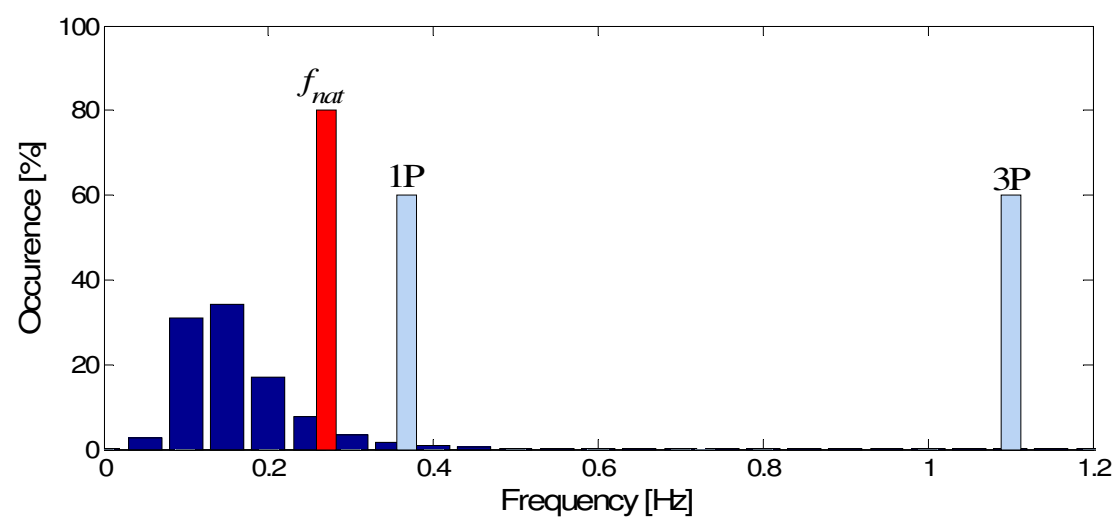

Figure 2.48 Occurrence of wave frequencies with plotted 1P and 3P frequencies for properties of a 3 bladed turbine after Opti-OWECS off the Dutch coast

\subsubsection{Compensating circumstances}

As shown in the previous sections, the design aim would be to create a soft-soft support structure, because it uses less steel and is therefore more const-effective. The trend for the natural frequency of the support structure indeed seems to be moving to this soft range when applying larger rotors and structures and variable speed turbine, with a major risk of resonant behaviour due to wave excitation. In this connection there are two important phenomena that play a role: aerodynamic damping and controllability of variable speed turbines.

\section{Aerodynamic damping}

It was shown in section 2.7.3 that when a typical soft-soft support structure is designed to prevent resonant excitation by the $1 \mathrm{P}$ frequency of the rotor, it would encounter waves with frequencies near its natural frequency for some $10 \%$ of the time (see Figure 2.48). Although resonant behaviour will occur, the dynamic response is significantly reduced as the wind loading on the rotor adds damping to the system, which considerably reduces the height of the peak in Figure 2.43. The tower top displacement and the total fatigue damage are thus correspondingly reduced. However, this aerodynamic damping is not present when the turbine does not produce energy (i.e. when blades are idling or parked). It has been calculated in [34] that, when compared to 
a parked turbine, the fatigue life of the Opti-OWECS support structure will be doubled when the turbine is in operation.

\section{Variable speed turbines}

Variable speed turbines are equipped with sophisticated controls to keep the system running at optimum speed. Although the variability of the rotation speed narrows the intervals of "safe" natural frequencies for the support structure, the controller can be used to create new intervals. Even though the natural frequency lies in the range of the rotational frequency band, the controller can be programmed to skip rotational frequencies around the natural frequency. This will prevent the rotor from exciting the tower's natural frequency. The tuning of the controller can best be done after installation and measurement of the actual first natural frequency of the support structure, because uncertainties in soil conditions and installation particularities can cause the actual natural frequency to deviate appreciably from the design [57]. This frequency skipping by the control system has been applied successfully at the Utgrunden Wind Farm in Sweden [4].

\subsection{Basic fatigue considerations}

\subsubsection{Introduction}

Fatigue is the process of gradual damage done to materials when these are subjected to continually changing stresses. Due to these stress changes, the material slowly deteriorates, initiating cracks which will eventually lead to breaking of the material. Offshore wind turbines are by default subjected to loads varying in time from wind as well as waves. This means that the stress response will also vary continuously, making offshore wind turbinesprone to fatigue if nothing else. In comparison to another highfatigue system, the aeroplane, a designer once stated: "During its lifetime, a wind turbine blade receives the equivalent of the loading of a World War II bomber doing continuous loopings $50 \mathrm{~m}$ of the ground for 30 years" [35]. Such observations do not only apply to the blades but also to the support structure, only these days it would be an A380 with double wing span $100 \mathrm{~m}$ of the ground.

This section gives an overview of the basic principle of fatigue and how fatigue is treated in design calculations.

\subsubsection{Fatigue of a teaspoon}

When we take an ordinary teaspoon, fatigue can be demonstrated rather easily. By bending the teaspoon back and forth, the spoon will break after 50-odd repeated bends, as shown in Figure 2.49. 


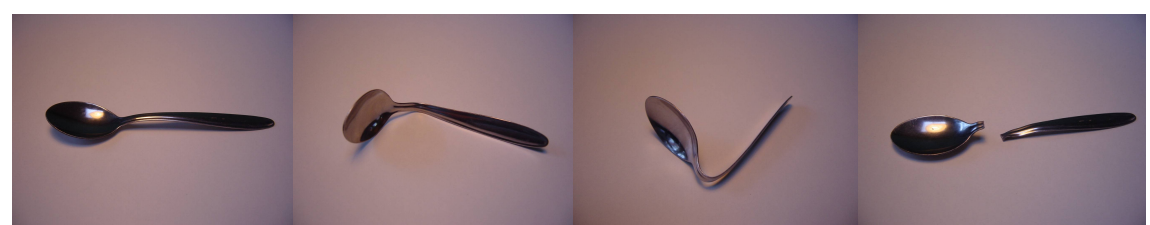

Figure 2.49 Unbent spoon, bending back and forth until breaking

The breaking is not caused by high tension put on the spoon: it is rather impossible to pull the material to its breaking limit manually. The breaking is caused by fatigue. Every new bending induces small cracks or causes existing cracks to grow. Figure 2.50 shows a close-up of the part with small cracks after such bending.

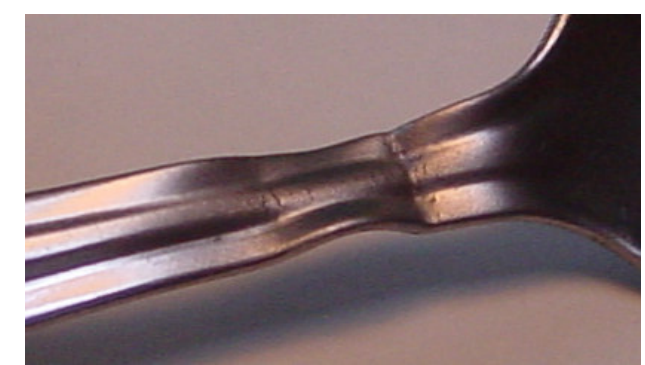

Figure 2.50 Close-up of bending area showing fatigue cracks appearing

Although this method of demonstration is very clear, fatigue will not only occur when a spoon (or structure) is bent through the plastic limit actually deforming the spoon. Figure 2.51 shows the stress-deformation curve of a typical metal. The left hand side shows a linearly increasing deformation with increasing stress following Hooke's law $\sigma_{s}=E \varepsilon_{s}$ [36]. When we come to the point marked $\sigma_{y}$ for yield stress, the material will start to yield: the deformation increases at more or less the same stress. After releasing the stress, this deformation will remain permanent as in the case of the bent tea spoon. When applying higher stresses than the yield stress, the material generally will be able to endure a higher stress until it breaks.

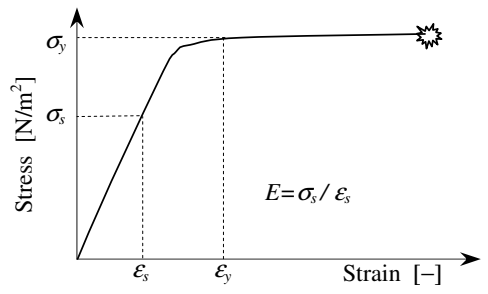

Figure 2.51 Typical stress-deformation curve for steel 
In the case of the teaspoon, fatigue is induced by continuously loading the spoon beyond the yield stress. In the case of offshore wind turbine support structures, the fatigue process is only acting in the lower end of the linear curve where both deformations and stresses are low. But when these small stresses are applied a sufficient number of times, fatigue will still occur.

\subsection{3 $S$ - $N$ curve and Miner rule}

To be able to take fatigue into account in the design process, an empirical design method for the design of steel structures is commonly used. First, for a typical structural detail or a structural connection an $S-N$ curve is created. The component is fixed in a testing frame and sinusoidal stress variations are applied to the component. By counting the number $N$ of cycles required to fail the component at the applied stress range, a single point on the failure envelope can be determined. For welded specimens it is only the stress variation that counts. The mean stress is unknown and has been shown not to influence the number of cycles to failure to any appreciable degree. Therefore the fatigue loading is expressed by the stress range $S$ rather than the stress level.

When the tests are repeated for a large number of identical specimens under different stress ranges $S$, the full failure envelope can be determined. As the empirical data show appreciable scatter, the design $S$ - $N$ curve is determined as the mean of the data minus two standard deviations in $N$, as shown in Figure 2.52.

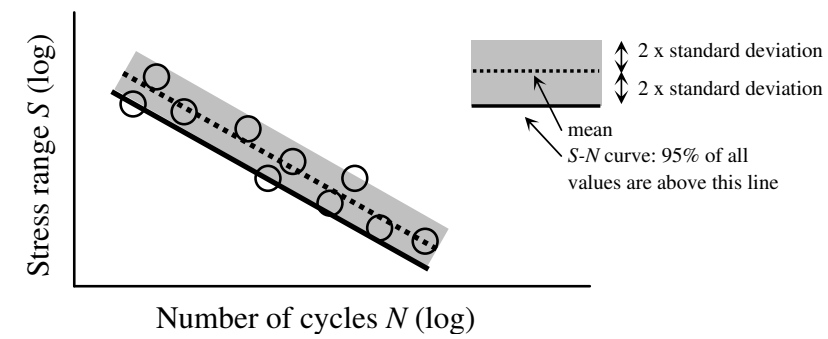

Figure 2.52 Failure points at stress range $S$ in $N$ number of cycles and the corresponding $S$ - $N$ curve

Design standards like those of Germanischer Lloyd and DNV include a number of prescribed $S-N$ curves for different details. Normal $S-N$ curves for steel usually have a branch with slope 3 and for higher cycles a branch with a slope 5 or sometimes 4 (depending on the detail and design standard used), both in the log-log domain. A typical $S$ - $N$ curve is shown in Figure 2.53. Safety factors can be applied to tailor the generic curve for components in special circumstances such as without corrosion protection, at uninspectable places, etc. 


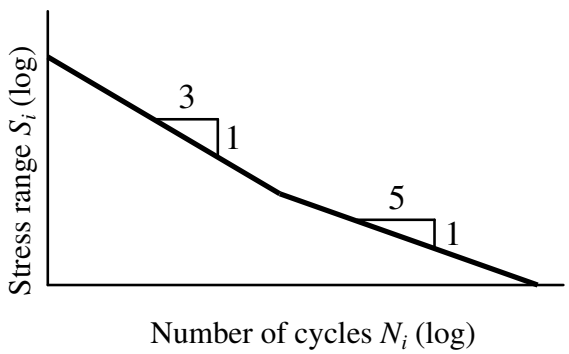

Figure 2.53 Typical $S$ - $N$ curve for a structural detail with slopes 3 and 5 on log-log scales

When the $S$ - $N$ curve for the detail under consideration is known, calculations of the stresses that the detail will experience during its lifetime should be performed. When all stress variations are known, they can be binned in number of variations $n_{i}$ per stress range class $S_{i}$. Taking the associated maximum allowable number of stress variations $N_{i}$ for each stress range class $S_{i}$ from the $S$ - $N$ curve, the Palmgren-Miner rule, or just Miner rule, can be applied [37]. This rule states that the cumulative fatigue damage $D_{f a t}$ is equal to the sum of $n_{i}$ over $N_{i}$ for all stress range classes:

$$
D_{f a t}=\sum_{i} \frac{n_{i}}{N_{i}}
$$

The Miner rule states that the detail will not fail due to fatigue if $D_{f a t}<1.0$.

\subsubsection{Counting methods}

The fatigue calculation method for variable stress ranges in the time domain can be summarized by the flowchart in Figure 2.54. Calculation of the stresses experienced by the detail being considered under all possible load cases during the lifetime will result in a large number of stress time series. By filtering the number of stress variations for every stress range class, the Miner sum can be calculated to check whether $D_{f a t}<1.0$.

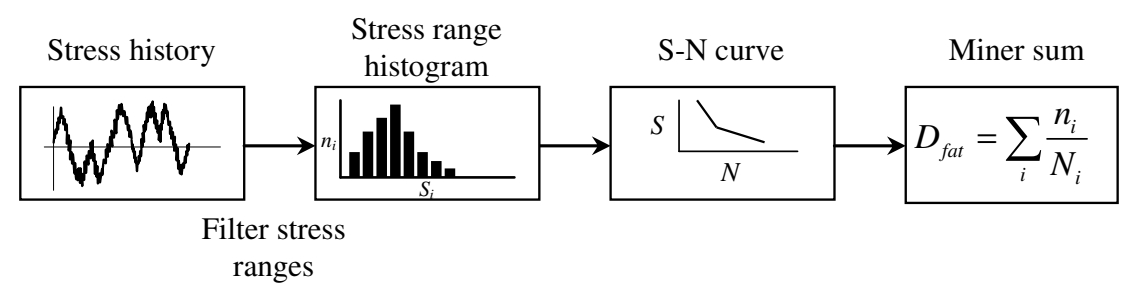

Figure 2.54 Flowchart of fatigue calculation due to variable stress ranges using $S$ - $N$ curve and Miner sum 
Not explained in Figure 2.54 is how to count the stress range cycles. For this counting, several methods exist. This thesis will not go into detail of the pros and cons of the different methods, but will only discuss methods and their field of application in the time and the frequency domain. The general procedure here is that the method most commonly used for a particular design methodology $\mathrm{f}$ be adhered to in this thesis.

\section{Peak counting}

When we consider a stress time record for the bending stress at some point of a typical offshore structure, we can determine the height of the stress peaks (the local maxima) above the mean stress between two successive crossings of the mean stress. A stress maximum is followed by a stress minimum between subsequent crossings of the mean stress. The stress range is now defined as the maximum minus the minimum stress. Alternatively, the stress range is defined as twice the maximum stress, even though the absolute value of the minimum stress is not necessarily equal to the maximum stress.

When post-processing a time series to determine the histogram of the number of variations $n_{i}$ per stress range class $S_{i}$, a commonly used method applying peak $\left(s_{i}\right)$ counting is:

- filter all peaks above the mean of the time record

- bin peaks to pre-set stress classes

- assume that every peak is associated with a stress trough of the same size and set the stress range at two times the peak stress

- the result is a histogram of stress range occurrences, see Figure 2.55.

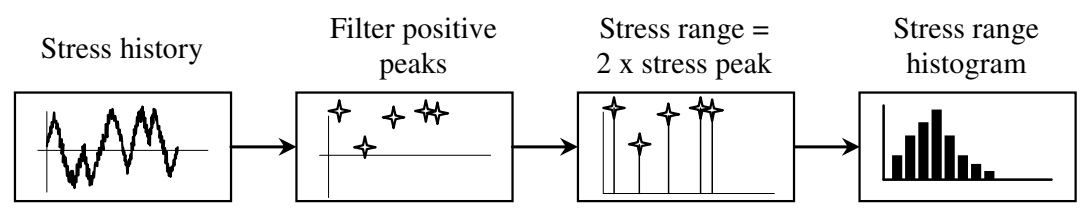

Figure 2.55 Flowchart for peak counting method

When we look at the bending stress at a point of the support structure of a typical offshore wind turbine we will not only find the characteristic stress record due to waves meandering around the mean stress level, but also higher frequency components induced by the turbine loads. Consequently, there can be several stress peaks between successive up- and down-crossings of the mean stress. If all peaks are taken into account, the peak counting method will yield a large number of stress ranges. If only the maxima between successive crossings are considered, peak counting results in a low number of stress ranges, see Figure 2.56. 

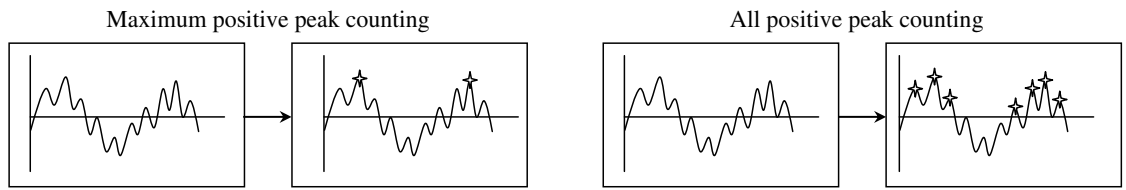

Figure 2.56 Applying maximum positive peak counting and all positive peak counting on a broad banded signal

\section{Rainflow Counting}

To take all peaks into account without doubling, the rainflow method was invented. Rainflow counting [70] [71] received its name because the method resembles rain flowing off a pagoda roof as shown in Figure 2.57. When the stress time series is rotated 90 degrees, the counting algorithm starts:

- at the beginning of the series (point 1)

- at every peak where a previous rainflow drops away (e.g. points $2,4,6, \ldots$ )

- at every trough (e.g. points $3,5,7, \ldots$ )

Every rainflow stops:

- at the end of the signal (point 13)

- when it encounters the bottom of a trough (e.g. series 2-3)

- when it encounters a flow from above (e.g. series 3-2')
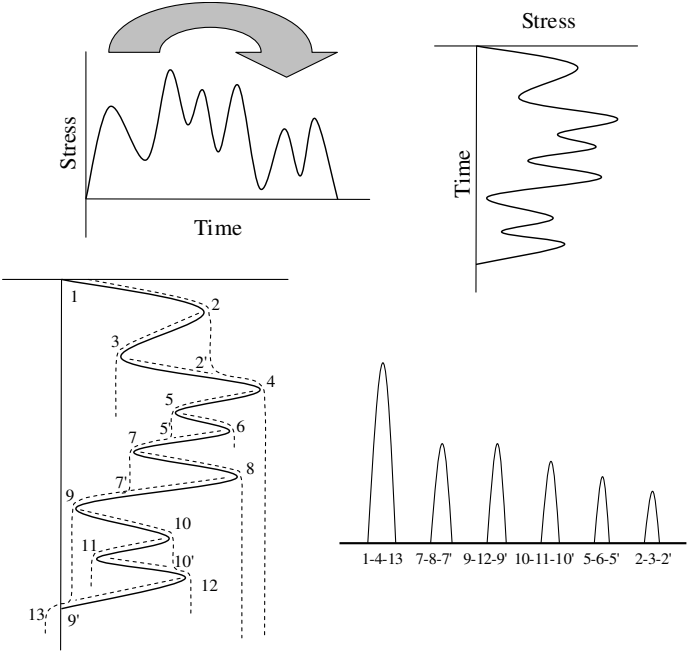

Figure 2.57 Rainflow counting illustration with beginnings and ends following the rainflow rules

When the method has been executed, the signal is taken apart in a number of half stress range variations: the rainflow cycle runs only in one direction each time. The method then combines pairs of half stress cycles: e.g. 1-4 and 4-13 become 1-4-13. The 
only difference between alternative rainflow methods is in the appreciation of the leftovers after combining cycle pairs, but this will not be detailed here. The rainflow method has now returned the stress range cycles with number of occurrences which can again be used in combination with the $S$ - $N$ curve to calculate the Miner sum.

\section{Frequency domain methods}

As discussed in section 2.3, time varying signals can be depicted as time series but can also be captured in a spectrum. When a spectrum is created of the bending stress, several methods exist to determine the stress variations from the spectral properties. All the methods incorporate the same basic steps:

- determine a probability distribution for the stress peaks (the local maxima), based on spectral parameters from the stress spectrum;

- $\quad$ stress ranges are assumed to be twice the peak stress and the distribution of stress ranges is assumed to be equal to the distribution of stress peaks;

- determine the total number of peaks or ranges occurring in the time period under consideration.

Let $s(t)$ be an arbitrary time series of a time varying stress and let $y$ represent the peaks $s_{i}$ (local maxima) of $s(t)$, ordered by increasing values such that $s_{i+1}>s_{i}$. Let further $P\left(y<s_{i}\right)=P\left(s_{i}\right)$ be the cumulative probability distribution of a stress peak being smaller than $s_{i}$. The distribution of the stress ranges of the time varying stress $s(t)$ is then also $P\left(s_{i}\right)$. The corresponding stress range class $S_{i}$ is defined by:

$$
S_{i}=2\left[\frac{1}{2}\left(s_{i}+s_{i+1}\right)\right]=s_{i}+s_{i+1} \quad i=0,1, \ldots \ldots
$$

The probability that $S_{i}$ occurs is:

$$
P\left(s_{i+1}\right)-P\left(s_{i}\right)
$$

The total number of stress ranges occurring during the stationary condition (state) under consideration is:

$$
\frac{T_{d}}{T_{s}}
$$

where $T_{d}$ is the duration of the state and $T_{s}$ is the average period between stress ranges. The number of occurrences of stress range class $S_{i}$ during this state is hence: 


$$
n_{i}\left(S_{i}\right)=\frac{T_{d}}{T_{s}}\left[P\left(s_{i+1}\right)-P\left(s_{i}\right)\right]
$$

The allowable number of stress range class $S_{i}$ according to the $S$ - $N$ curve is $N_{i}$, so that the relative damage contribution of stress range class $S_{i}$ is:

$$
\frac{n_{i}\left(S_{i}\right)}{N_{i}\left(S_{i}\right)}
$$

According to the Miner rule, the partial fatigue damage done during the state under consideration is then:

$$
D_{\text {fat }, \text { state }}=\sum_{i} \frac{n_{i}\left(S_{i}\right)}{N_{i}\left(S_{i}\right)}
$$

The total fatigue damage during the entire service life (or any other desired period) is next determined by summing the partial damages for all states:

$$
D_{\text {fat,life }}=\sum_{\text {all states }} D_{f a t, \text { state }}
$$

What remains is to specify theoretical results for $P\left(s_{i}\right)$ and $T_{s}$, for which two options exist.

If the time series $s(t)$ is Gaussian and narrow-banded the stress peaks are theoretically distributed according to the Rayleigh distribution. Due to the narrow-banded nature of $s(t)$ the Rayleigh distribution also applies to stress ranges. Furthermore, the average period between stress peaks as well as between stress ranges is the average zerocrossing period: $T_{s}=T_{z}$. If $s(t)$ is broad-banded there are intermediate stress peaks between zero-crossings and even negative stress peaks (local maxima below the mean (see right-hand side of Figure 2.56)). The theoretical distribution for all peaks, including the negative ones, is now the Rice distribution. In dealing with broad-banded time series two options are commonly used as approximations. The first is to apply the Rayleigh distribution and $T_{s}=T_{z}$, notwithstanding the broad-banded nature. This may be considered as being equivalent to the maximum positive peak counting between zerocrossings, as shown on the left-hand side of Figure 2.56, The second is to ignore negative stress peaks as being physically unrealistic and apply the Rice distribution to describe all positive peaks. This may be considered as equivalent to all positive peak counting for time series, as shown in the right-hand side of Figure 2.56. Stress ranges 
are set at twice the positive peaks and the Rice distribution is assumed to describe the distribution of stress ranges. The average period between stress peaks and between stress ranges is now the average crest period: $T_{s}=T_{c}$. Both options are detailed hereafter.

\section{Rayleigh}

The Rayleigh distribution is based on the zeroth moment of the spectrum only, $m_{0}$ :

$$
P_{\text {Rayleigh }}\left(s_{i}\right)=1-e^{-\frac{s_{i}^{2}}{2 m_{0}}}
$$

Equation (2.47) can be rewritten in the stress range $S_{i}=2 s_{i}$ to become:

$$
P_{\text {Rayleigh }}\left(S_{i}\right)=1-e^{-\frac{S_{i}^{2}}{8 m_{0}}}
$$

The parameter $m_{0}$ is the zeroth moment of the spectrum of the time series $s(t)$ and the square of the standard deviation of $s(t)$. Using equations (2.43) (2.48) and $T_{z}$ from table 2.1 the number of stress ranges per stress range class becomes:

$$
\begin{aligned}
n_{i}\left(S_{i}\right) & =\frac{T_{d}}{T_{z}}\left[P_{\text {Rayleigh }}\left(S_{i+1}\right)-P_{\text {Rayleigh }}\left(S_{i}\right)\right] \\
& =T_{d} \sqrt{\frac{m_{2}}{m_{0}}}\left[e^{-\frac{S_{i}^{2}}{8 m_{0}}}-e^{-\frac{S_{i+1}^{2}}{8 m_{0}}}\right]
\end{aligned}
$$

\section{Rice}

The Rice distribution [72] [73] is based on the zeroth, second and fourth order moments of $s(t)$. Using the spectral width parameter $\varepsilon$ :

$$
\varepsilon=\sqrt{1-\left(\frac{T_{c}}{T_{z}}\right)^{2}}=\left[1-\frac{m_{2}^{2}}{m_{0} m_{4}}\right]^{1 / 2}
$$

the Rice distribution is given by: 


$$
P_{\text {Rice }}\left(\frac{1}{2} S_{i}\right)=P_{\text {Rice }}\left(s_{i}\right)=P_{N}\left(\frac{s_{i}}{\varepsilon \sqrt{m_{0}}}\right)-\sqrt{1-\varepsilon^{2}} \cdot e^{-\frac{s_{i}^{2}}{2 m_{0}}} \cdot P_{N}\left(\frac{s_{i} \sqrt{1-\varepsilon^{2}}}{\varepsilon \sqrt{m_{0}}}\right)
$$

where $P_{N}$ is the cumulative probability of the standard normal distribution:

$$
P_{N}(x)=\frac{1}{\sqrt{2 \pi}} \int_{-\infty}^{x} e^{-\frac{1}{2} t^{2}} d t
$$

With equations (2.43), (2.51) and $T_{c}$ from table 2.1 the number of stress ranges per stress range class now becomes as per equation (2.53):

$$
\begin{aligned}
& n_{i}\left(S_{i}\right)=\frac{T_{d}}{T_{c}}\left[P_{\text {Rice }}\left(S_{i+1}\right)-P_{\text {Rice }}\left(S_{i}\right)\right] \\
& =T_{d} \sqrt{\frac{m_{4}}{m_{2}}}\left[P_{\text {Rice }}\left(S_{i+1}\right)-P_{\text {Rice }}\left(S_{i}\right)\right]
\end{aligned}
$$

\section{Dirlik}

As rainflow counting is the standard in the wind industry these days, a frequency domain approximation of the rainflow ranges has long since been investigated. The most accurate, when compared to time domain rainflow counting of the same event, is the Dirlik method [74]. This method is completely empirical. By running large numbers of broad-band signals in both time and frequency domain, the parameters in the Dirlik formula were tuned. No theoretical background exists.

In a recent paper [75] the effectiveness of Dirlik, as compared to time domain rainflow counting, was again proven and a new, more theoretically supported method was also introduced. These two methods give very similar results. The latter method has not been used in this thesis, but might prove useful in the future.

Equations (2.54) and (2.55) give the Dirlik formula.

$$
P_{\text {Dirlik }}\left(S_{i}\right)=\frac{\frac{D_{1}}{Q_{D}} e^{-\frac{Z_{D}}{Q_{D}}}+\frac{D_{2} Z_{D}}{R_{D}{ }^{2}} e^{-\frac{Z_{D}{ }^{2}}{2 R_{D}{ }^{2}}}+D_{3} Z_{D} e^{-\frac{Z_{D}{ }^{2}}{2}}}{2 \sqrt{m_{0}}}
$$

with: 


$$
\begin{aligned}
& Z_{D}=\frac{S_{i}}{2 \sqrt{m_{0}}} \quad x_{m}=\frac{m_{1}}{m_{0}} \sqrt{\frac{m_{2}}{m_{4}}}=\frac{T_{c}}{T_{m}} \quad \gamma_{D}=\frac{m_{2}}{\sqrt{m_{0} \cdot m_{4}}}=\sqrt{1-\varepsilon^{2}}=\frac{T_{c}}{T_{z}} \\
& D_{1}=\frac{2\left(x_{m}-\gamma_{D}^{2}\right)}{1+\gamma_{D}^{2}} \quad D_{2}=\frac{1-\gamma_{D}-D_{1}+D_{1}^{2}}{1-R_{D}} \quad D_{3}=1-D_{1}-D_{2} \\
& Q_{D}=\frac{1.25\left(\gamma_{D}-D_{3}-D_{2} \cdot R_{D}\right)}{D_{1}} \quad R_{D}=\frac{\gamma_{D}-x_{m}-D_{1}^{2}}{1-\gamma_{D}-D_{1}+D_{1}^{2}}
\end{aligned}
$$

The number of stress ranges per period under investigation is again found using the crest period $T_{c}$ :

$$
\begin{aligned}
& n_{i}\left(S_{i}\right)=\frac{T_{d}}{T_{c}}\left[P_{\text {Dirlik }}\left(S_{i+1}\right)-P_{\text {Dirlik }}\left(S_{i}\right)\right] \\
& =T_{d} \sqrt{\frac{m_{4}}{m_{2}}}\left[P_{\text {Dirlik }}\left(S_{i+1}\right)-P_{\text {Dirlik }}\left(S_{i}\right)\right]
\end{aligned}
$$

\section{Overview of time and frequency domain methods}

Figure 2.58 gives an overview of the methods described in this section as used in both the frequency and the time domain.

Time Domain
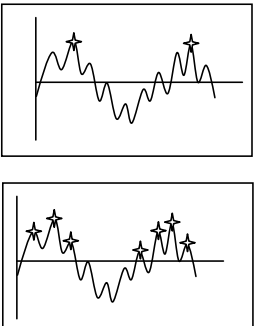

All positive peak counting

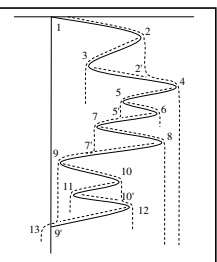

Frequency Domain
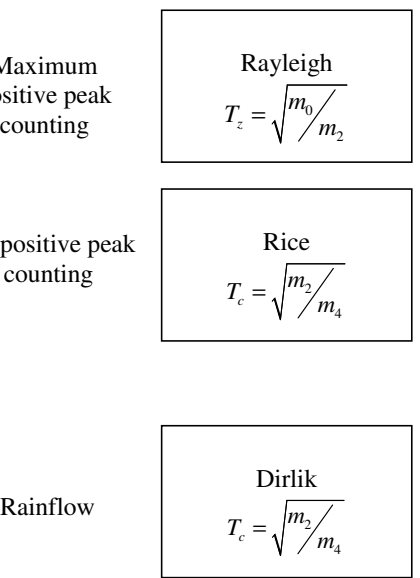

Figure 2.58 Overview of determining stress ranges in time and frequency domain 


\subsection{Foundations}

\subsubsection{Soil properties}

The build-up of the seabed is the result of tens of thousands of years of geological and morphological processes. In the North Sea, large parts of the seabed consist of layers of clay and sand. Both soil types are characterised in capacities of loose, medium, dense to very dense for sand and soft, stiff to hard for clay. Figure 2.59 shows three offshore locations and the different layers of soil [57].

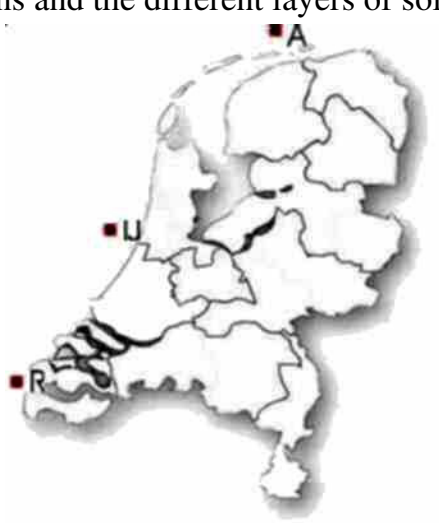

Location

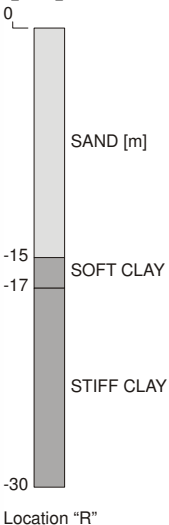

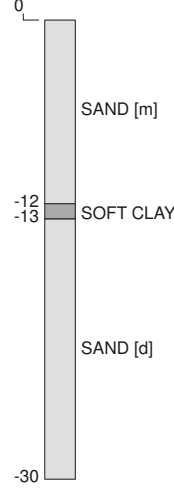

Location "IJ"

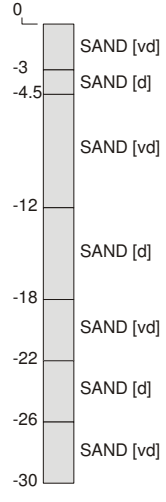

Location "A"

Figure 2.59 Soil layers for 3 offshore sites with medium [m], dense [d] and very dense [vd] sand layers and soft and stiff clay [57]

The characterisation of loose to dense sand and soft to hard clay only gives a first indication of the ability of the soil to carry load. For design, more detailed knowledge is required. This is usually gathered through in-situ sampling and analysis of drilled samples in the laboratory. The first property measured for all types is the density $\rho_{\text {soil }}$ $\left[\mathrm{kg} / \mathrm{m}^{3}\right]$, usually for submerged soil, which is the dry density minus the density of water. A typical value is between 0.4 and $1.0 \mathrm{~kg} / \mathrm{m}^{3}$. For clay, the undrained shear strength $s_{u}$ and the strain at $50 \%$ of the maximum stress $\varepsilon_{50}$ are measured. Table 2.6 gives an overview of typical values when no reliable soil data is available.

Table 2.6 Characteristic parameters for clay

\begin{tabular}{l|c|c}
\multicolumn{1}{c|}{ Clay type } & $s_{u}[\mathrm{kPa}]$ & $\varepsilon_{50}[\%]$ \\
\hline \hline soft & $0-25$ & 1.5 \\
firm & $25-50$ & 1.5 \\
stiff & $50-100$ & 1.0 \\
very stiff & $100-200$ & 0.5 \\
hard & $>200$ & 0.5
\end{tabular}


For sand the friction angle $\phi^{\prime}$ and the relative density of sand $D_{r}$ are derived directly from in-situ measurements. The initial modulus of horizontal subgrade reaction, $k_{s}$, can then be found with the graph in Figure 2.60 [19].

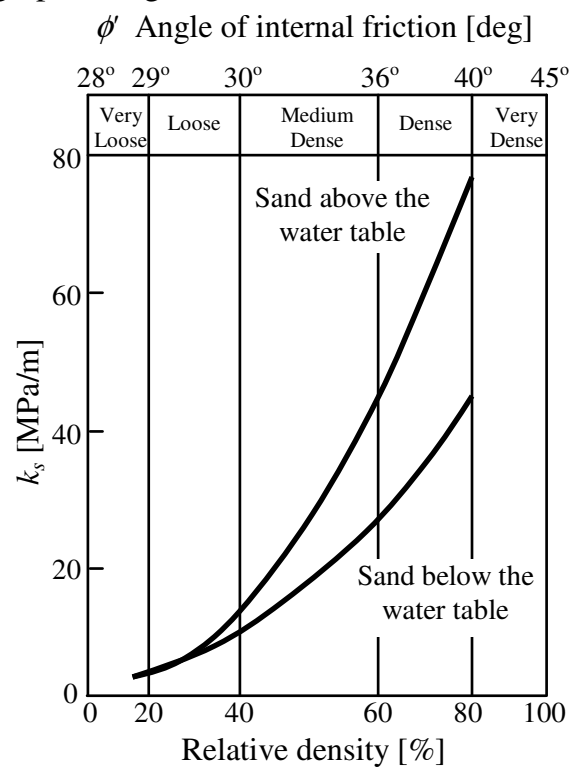

Figure 2.60 Initial modulus of subgrade reaction $k_{s}$ as function of friction angle $\phi^{\prime}[19]$

A good standard for soil investigation and characterisation of soil properties at offshore sites is ISO 19901-4 [76], a document in the ISO 19900 series of new offshore structure standards.

\subsubsection{Foundation modelling}

\section{Transfer of horizontal loads, vertical loads and moments}

In the design of offshore support structures, two main directions of load transfer must be analysed. First, the foundation must be able to transfer all vertical loads, the weight of the structure, to the soil. This is mainly done by friction: the soil around the pile takes a small load per area of surface and as long as the load-transfer-area is large enough, the foundation will suffice. Next to the friction on the outside of the pile, the steel rim of the pile and the soil plug inside the pile may also give vertical bearing capacity. For monopiles of $4 \mathrm{~m}$ diameter and more, the extra pile plug resistance is usually not taken into account due to the large diameter of the pile.

For overturning moments, multi-legged structures mainly rely on vertical capacity. Due to the fixation in the frame, the piles of a multi-legged structure deform in an Sshape, mobilising at the same time horizontal soil resistance. The overturning moment 
is then transferred as axial loads to opposing foundation piles as shown in Figure 2.61. For monopiles, all horizontal loads and moments must be transferred directly to horizontal soil reactions, as shown in the right-hand side of Figure 2.61. As the pile is not fixed at the top, it is free to rotate and translate. For offshore wind turbines on monopiles, this horizontal load transfer usually dictates the pile length: the pile must be long enough to mobilise enough soil over its length to transfer all loads and prevent "toe kick": displacement of the tip of the pile.

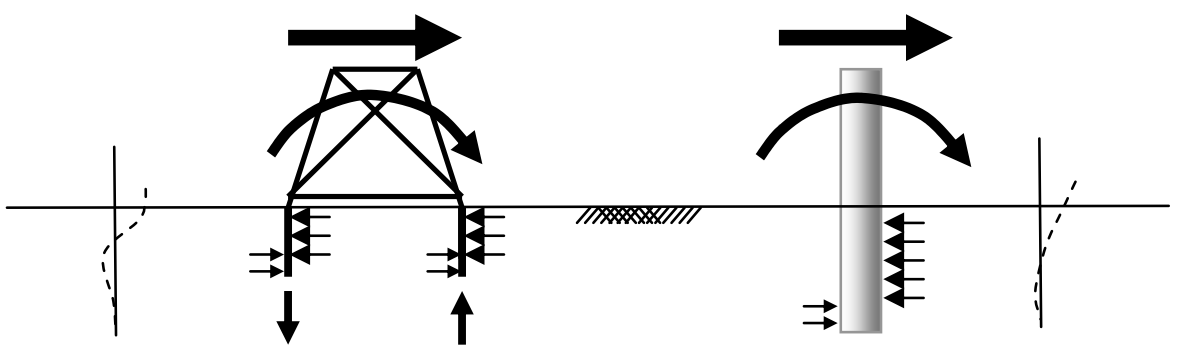

Figure 2.61 Transfer of horizontal loads and moments in multi- and monopile structures and a schematic representation of the pile deformation

\section{Soil springs}

To model the soil reaction loads a set of soil springs is used. Figure 2.62 shows the springs for the horizontal and vertical direction as well as for the pile plug [57].

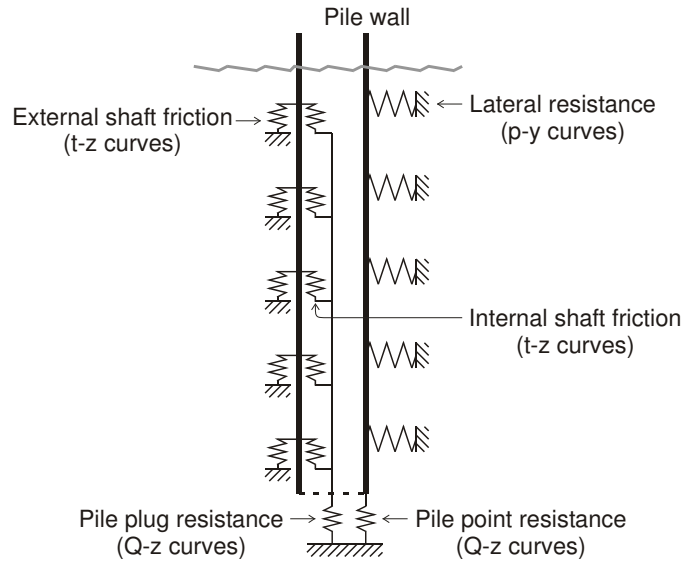

Figure 2.62 Spring model of pile-soil interaction [57]

All springs in Figure 2.62 are non-linear. The properties can be derived from site measurements through calculation methods prescribed in the standards [52], [77]. The 
typical shape of these curves is shown in Figure 2.63. For the first part, left-hand side, the soil reacts linearly and elastically; when the load is released, the soil will return to its original state. Beyond a break point in the curve, deformations will become permanent and the soil starts to lose resistance.

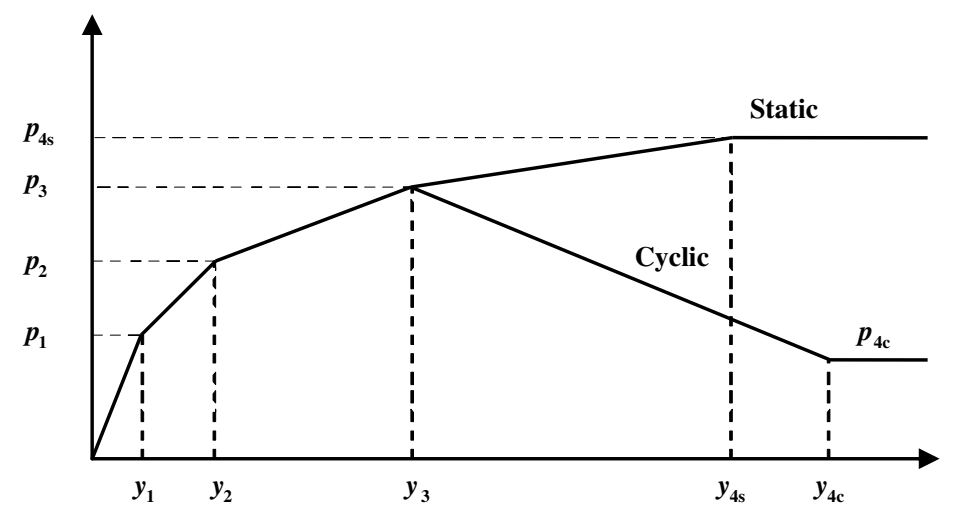

Figure 2.63 Typical shape of horizontal load $p$ and displacement $y$ in a $p-y$ curve for soft clay under static and cyclic loading according to Matlock [68]

For extreme load cases and foundation design, the full non-linear model must be used. For load calculations of the offshore wind turbine and its support structure, a reduced model can also be used as described next.

\section{Stiffness matrix model for foundation representation}

The non-linear spring model can be created in a straightforward way in a finite element program. For fatigue load simulations of the offshore wind turbine however, the full complexity of the non-linear systems is usually not required: most soil reactions remain within the linear elastic range. To reduce calculation time, a stiffness matrix model can be used, which has shown excellent agreement with full non-linear models [57].

The foundation properties are represented by two coupled springs for lateral and rotational reactions as shown in Figure 2.64. 

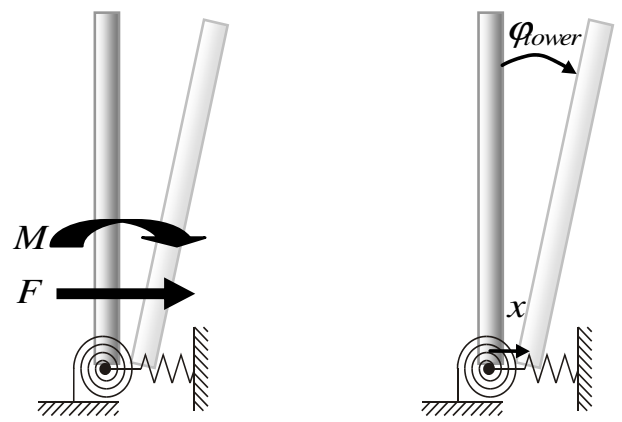

Figure 2.64 Coupled lateral and rotational springs for foundation representation with loads $F$ and $M$ and reactions $x$ and $\varphi_{\text {tower }}$

The spring properties are derived by applying two load sets that are typical for operational conditions of the offshore wind turbine to the non-linear model and using the outcome to derive the spring constants in:

$$
\left[\begin{array}{c}
F \\
M
\end{array}\right]=\left[\begin{array}{ll}
k_{x x} & k_{\varphi x} \\
k_{x \varphi} & k_{\varphi \varphi}
\end{array}\right]\left[\begin{array}{c}
x \\
\varphi_{\text {tower }}
\end{array}\right]
$$

Although other models exist, the non-linear $p-y$ model for foundation detailing and the coupled spring model for offshore wind turbine load cases were proven to be the most suitable for offshore wind turbine design.

For more details on these and other models and comparison with measurements on offshore wind turbines, the reader is referred to [57] [58]. 


\section{Differentiating integrated design}

\subsection{Introduction}

Offshore wind turbine design spans a wide range of disciplines. To come to an optimal and cost-effective final design, previous studies [4][5] have stressed the importance of designing the offshore wind turbine as an integrated system: all sub-components should be taken into account and simultaneously optimised as a whole, rather than doing this in separate (usually discipline dependent) portions. Although this general statement of requiring integrated design is true and crucial for the final design check, design practice is not always easily moulded into explicit integration, especially not in the preliminary design stages. This chapter therefore aims at identifying steps within the integrated process that can be treated in a more or less separate manner. Eventually these differentiated steps will serve the final integration of the overall design.

Design documents for the wind turbines at the four reference sites described in chapter 1 are used to investigate the basic set-up of the overall design process. As a reference, a design document for an offshore oil \& gas structure has also been investigated. The documents and project summaries are presented in section 3.2. The main steps of the design process are further detailed with regard to assessment of the environment in 3.3. Critical design details are discussed in 3.4. Section 3.5 gives a general overview of the ultimate limit state checks required for offshore wind turbines. In section 3.6 the fatigue limit state calculations are analysed. All design reports follow a different approach with respect to fatigue analyses. Section 3.7 therefore summarizes the advantages and disadvantages of the approaches used and contains a proposal for a more consistent and understandable method of assessing fatigue.

\subsection{Hands-on design experience}

\subsubsection{Available documents for reference}

As the number of offshore wind farms designed by a single company can to date still be counted on one hand (or finger), the amount of readily available information is normally limited to what can be found in the literature, meaning essentially reports of research and development projects funded by governments.

To put the documents in a proper perspective, a time line is shown in Figure 3.1. The period during which each of the four wind farms was designed, built and commissioned is shown by a blue bar. Similarly, the periods during which relevant research projects were carried out and the reports were published are shown by green bars. Finally, the 
dates of publication or expected publication of design standards are indicated by the red diamonds.

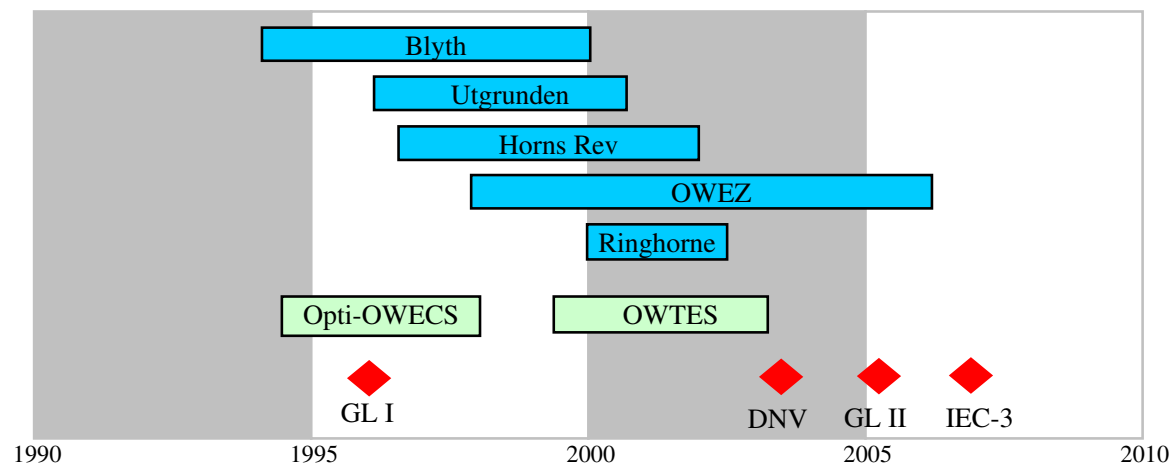

Figure 3.1 Time line for 4 offshore wind farms (blue), research projects (green) and publication of design standards

The following documents were available for review for this thesis project.

\section{Opti-OWECS}

This EU sponsored research project resulted in a first "new-fashion" approach to designing a $300 \mathrm{MW}$ offshore wind farm. The site selected is almost equal to the OWEZ site off the coast of Egmond in The Netherlands. Water depth is $20 \mathrm{~m}$ and a 3 MW, 2 bladed, $80 \mathrm{~m}$ diameter rotor, pitch regulated constant speed turbine was used for the design. The support structure designed in the project was a monopile with a diameter of $3.5 \mathrm{~m}$ reducing to $2.8 \mathrm{~m}$ from the platform up, with a hub height of $60 \mathrm{~m}$ above mean sea level.

The project resulted in 6 sub-reports of which report no. 4 is most relevant to this chapter, describing the design of the offshore wind turbine [5].

\section{Blyth, OWTES}

The turbines at Blyth were the subject of a large EU funded project: OWTES. The project resulted in several reports relevant to this thesis [3][47][50][51][57]. Furthermore, design documents were made available by AMEC [38], but without numbers: only the design methodology is revealed. Shell supplied the environmental study report [56].

\section{Utgrunden}

For the Utgrunden project, the Section for Wind Energy at the Delft University of Technology performed a preliminary design study in [39]. This document provides 
insight in the application of lessons learned during the Opti-OWECS project on a real offshore wind farm.

\section{Horns Rev}

"Mono Pile Foundation" by LIC Engineers A/S [40] is the first preliminary design study for Rødsand (now Nysted) and Horns Rev offshore wind farms. It covers the entire design process including proposed installation methods, time planning and decommissioning.

\section{OWEZ}

During the lengthy process of obtaining a license for the Offshore Wind farm Egmond aan Zee, the NEG-Micon NM92 turbine was a candidate for offshore application. The design report for the support structure was made available for this thesis [41]. The takeover of NEG-Micon by Vestas meant the end of production of the NM92 in 2004; this report is therefore the most up to date "design study" that includes the NM92 as part of a scientific study.

\section{Ringhorne structure}

As a reference for offshore wind turbine design, the design of the Ringhorne platform has been made available to this thesis project [42]. This document describes the basic design steps of a typical offshore oil \& gas structure, designed, constructed and installed by Heerema.

\section{Design standards}

The standards mentioned in the abbreviations in Figure 3.1 are:

- GL I: Gernamischer Lloyd design rules for offshore wind turbines [20], drafted in co-operation with the Opti-OWECS study

- DNV: Det Norske Veritas, Design of offshore wind turbine structures [19]

- GL II: Germanischer Lloyd, revised version of GL I, including lessons learned in the OWTES project [64],

- IEC-3: International Electrotechnical Commision standard IEC 61400-3, forthcoming international offshore wind turbine standard [65].

\subsubsection{Building blocks of design}

All design documents have a similar structure. First, a short introduction describes the project, the location and other particulars and introduces design particulars from previous documents. Then the codes and standards to be used are identified.

A significant part of all documents deals with the analysis of environmental parameters: wind, waves, current, soil, earthquake, etc. Next, details which are critical 
for the overall design are described. These details can be designed more or less independent of the overall structure, but their presence significantly influences the design of the overall structure. When these details have been incorporated, the structure can be checked for extreme load cases in the ultimate limit state and for fatigue.

The design steps are summarized in Figure 3.2 as building blocks of the design process. The different building blocks are detailed further in the following sections. The relating section number is shown in the blocks in Figure 3.2. Ofcourse, the design process is an iteration of the steps depicted here and will not lead directly to a final answer.

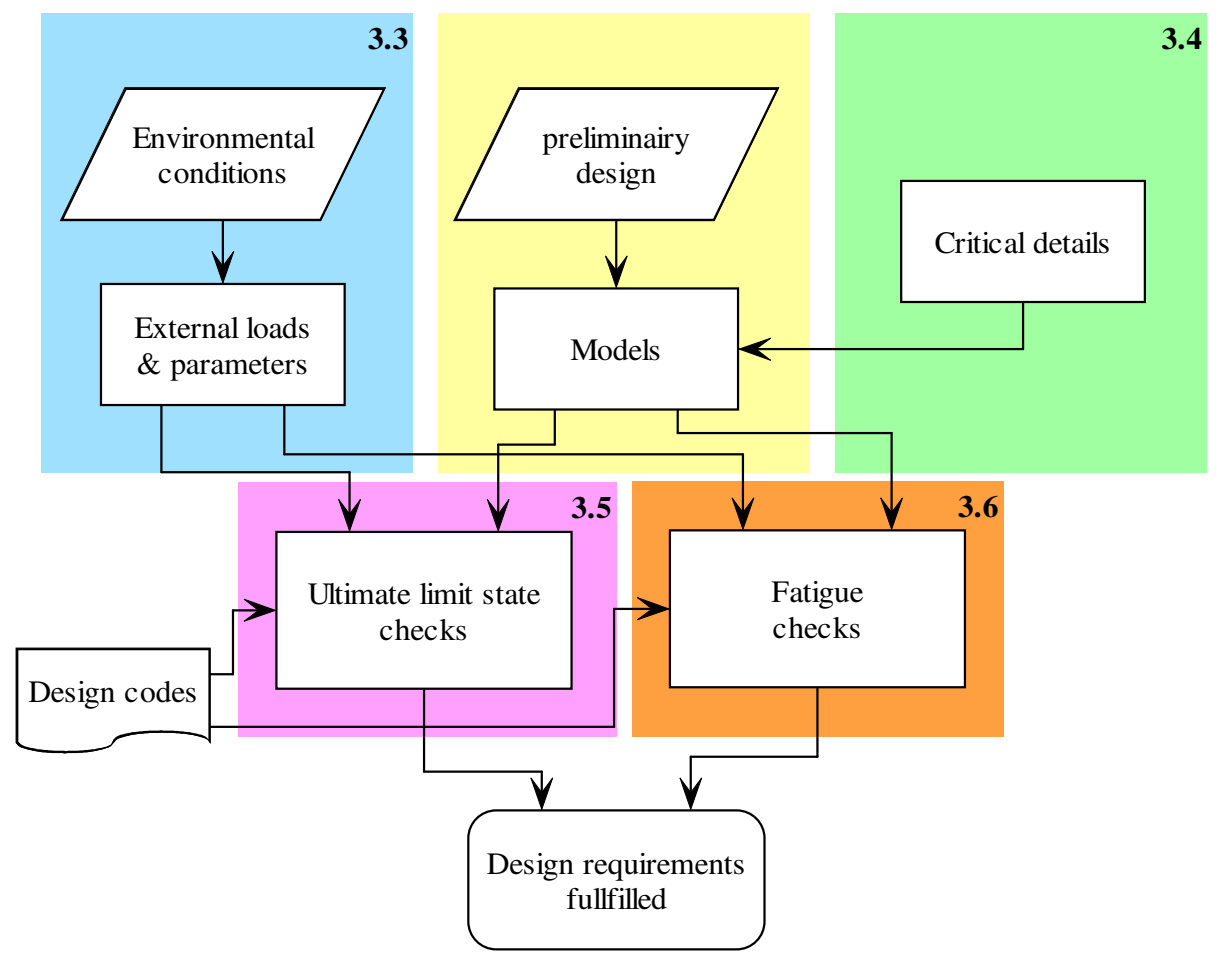

Figure 3.2 Building blocks of design

\subsection{Environmental conditions}

\subsubsection{Introduction}

The relationship between the environment and offshore wind turbines is rather unique: offshore wind turbines are especially designed to catch as much wind load (energy) as 
possible. However, the economical aspect of wind load and the impact of wind loads on structural design must be strictly separated: safety factors on potential profit are by no means translatable to structural safety. Nevertheless, both calculations are critical for a successful project and rely heavily on the quality of the environmental data available for a site. In this section, the critical parameters for the design of the support structure are discussed.

\subsubsection{Wind and wave data}

Wind and wave data can be obtained by direct measurements, by satellite observations or by the use of theoretical models for wind and wave fields. These options are briefly discussed in this section, together with which data was used in the various projects.

\section{Buoys}

Most countries bordering on a sea have a government agency which has measured the wave climate for many years. In the recent past these measurements have been made available to the public via internet. For instance, the Dutch Institute for Coastal and Sea Research (RIKZ) distributes their data on www.golfklimaat.nl. Most of this data is measured by wave buoys positioned along the coast. The data on this particular internet site ranges over a period of 20 years. For some locations, the sea state parameters (wave height, period, direction) are accompanied by wind parameters from a nearby meteorological station.

For design of offshore wind farms, the presence of a buoy in the area can significantly aid the design process. The freely available data can be processed easily to obtain a reasonably detailed description of the wave (and wind) climate. An offshore wind farm is, of course, not always situated at exactly the same location as the buoy. Special care must be taken when transforming the measured data to the wind farm site, also taking bathymetry features into account.

For the OWEZ project a thorough analysis has been performed of the data from the buoy marked YM6, on which the design was subsequently based. Measured data from a nearby met-mast on the pier of IJmuiden harbour was available for the same continuous 20 years.

In order to obtain better information on the on-site conditions, the OWEZ consortium subsequently installed a buoy at the planned location of the offshore wind farm. This buoy is not only capable of measuring wave height and period, but also the wind speed at $10 \mathrm{~m}$ above mean sea level. A picture of the buoy is shown in Figure 3.3.

\section{Measurements from a platform}

For more extensive measurements, the buoy network is usually extended with a platform. A platform offers the possibility to measure the wind speed at different heights, to test new equipment on site and to do experiments. Off the Dutch coast, 
Meetpost Noordwijk has been used for 3 decades. In 2003, the Germans installed a measurement platform in the North Sea off Borkum, FINO, solely for measurements to support their North Sea offshore wind farm design efforts; a photograph of the platform is shown in Figure 3.3.

\section{Met-mast}

As the first offshore wind farms were mainly developed to test the feasibility of offshore wind energy, all farms were equipped with met-masts: slender structures with anemometers reaching up to hub height or beyond to measure the offshore wind characteristics in the vicinity of the farm. Although met-masts are an excellent research tool, they are not ideal to gather detailed environmental data for design purposes. Their installation is usually not timely enough to gather data for a sufficiently long period to provide statistically sound data. Furthermore, the costs of over $€ 1$ million make it a very expensive instrument in an industry, which needs to cut costs as much as possible in order to become fully self-supporting. A picture of the Horns Rev met-mast is shown in Figure 3.3.
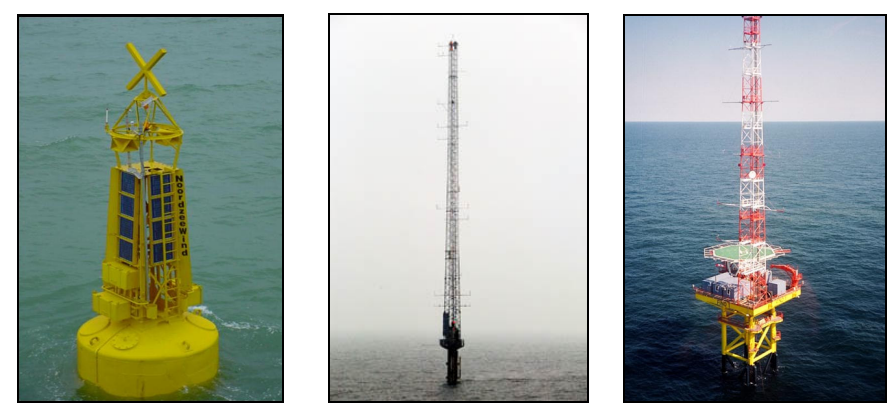

Figure 3.3 Wave and wind measurement buoy at OWEZ, met-mast at Horns Rev, FINO platform north of Borkum

\section{Hindcast data}

To support the design of their structures, the offshore oil \& gas industry in the countries around the North Sea initiated the NESS project (North European Storm Study) to create a wave database for the entire North Sea. A large mathematical model was created, incorporating all relevant land masses and wind-sea interaction. The model was fed with meteorological measurements from stations around the North Sea and validated with wind, wave and current measurements on platforms offshore. The model was used to create a database of wind, wave and current hindcast data for sections of 30 x $30 \mathrm{~km}$ covering the entire North Sea. As the project initially focussed on storms, 7 winters and 2 summers were included. While the project was carried out, 3 consecutive 
stormy summers made the project team decide to continue the hindcast modelling for another 5 full years, the NEXT study.

The database of NESS-NEXT data is currently only available to the project partners. It offers a highly detailed description of all important environmental parameters in a standard format for all locations in the North Sea. A minor drawback is in the limitation of the models used: they were mainly applied to the deeper areas of the North Sea. To use the data for current offshore wind farms, offshore to near-shore "transformation" of the data may in some cases be required.

\section{Satellite data}

A relatively new source of design data comes from satellite observations. By processing altimeter, scatterometer and SAR (radar) images of a large stretch of sea or ocean, wave data can be determined. The wind field data for the local winds driving the local waves can also be established. For instance, www.waveclimate.com offers 15 years of processed satellite data for all sea areas in the world. The service is extended with hindcast models, which are fed by both satellite observations and by available buoy and platform measurements to constantly improve and extend the total database. By using these hindcast models a large drawback of satellite data is overcome, which is that satellites only cover stretches of sea from time to time rather than continuously, thereby missing statistically important continuity.

The ease of access and the way in which the data is presented on the internet make it a very useful source for design data for preliminary design. As for many locations no other sources of such length exist, satellite data can also be usefully applied during later stages of the design process.

Recent developments have made satellite data compatible with wind farm and offshore wind turbine software to further enhance the design process [24].

\section{Data used for the reference sites}

\section{Opti-OWECS}

During the Opti-OWECS study, NESS-NEXT data was made available for this research project. As a database grid point coincided with the location under consideration for the example design application, no transformation of wave and wind data was needed. Seven full years of hindcast data were available for simultaneous wind, wave and current parameters.

\section{Blyth}

With Shell as partner in the project, the design of Blyth could be based on the NESSNEXT data. The problem for this site was that the nearest data point was several tens of kilometres further offshore in $40 \mathrm{~m}$ deeper water. The assistance of Fugro-Geos was called in to create an oceanographic model of the area, which was fed with the NESS- 
NEXT data and calibrated with several buoys along the coast within the model area. The complete design data document covered all wave and wind directions.

\section{Utgrunden}

As the Utgrunden report relates to a first exploration, very crude methods were used to derive correlated wind and wave climate data. The significant wave height of a 50 year sea state was established as $H_{s, 50}=4.5 \mathrm{~m}$. Then a Weibull distribution was assumed to determine the significant wave height distribution from 0 to $4 \mathrm{~m}$. Through a prescribed relationship between $H_{s}$ and $T_{z}$ for the Baltic [59], the zero-crossing period associated with each $H_{s}$ was added. Finally, a relationship between wave height and wind speed produced wind speeds per sea state.

\section{Horns Rev}

The preliminary design document used for this project was not based on any on-site measurements. Based on water depths retrieved from charts, the maximum wave height was established as the breaking wave height of $0.78 \mathrm{x}$ water depth. For wind speeds a Weibull distribution was used in combination with a standard maximum wind class. The document states that fatigue will be assumed to only be wind induced. In later design documents, wave and wind data from the met-mast and from wave buoys were used.

\section{OWEZ}

The OWEZ site nearly coincides with the Opti-OWECS area. A wave buoy is located less than $10 \mathrm{~km}$ west of the location. The freely available 20 year wave data combined with wind data from the pier of IJmuiden harbour were used to derive the design conditions.

\section{Ringhorne}

For the Ringhorne design, all wind and wave design data were supplied by the client. Based on an analysis of the NESS-NEXT database, all critical design data was summarized in a document. The major advantage of designing such a structure is that the design requirements are clear, making data gathering a straightforward job.

\subsubsection{Tides, currents and storm surges}

Contrary to wind and wave data, the tidal parameters can usually be retrieved more easily. Any sea chart will give both tidal range and tidal current velocities at several locations. Figure 3.4 shows the tidal ranges for the five reference sites and the current speeds at spring tide. The figure clearly shows the relationship between large tidal differences and high current velocities. 

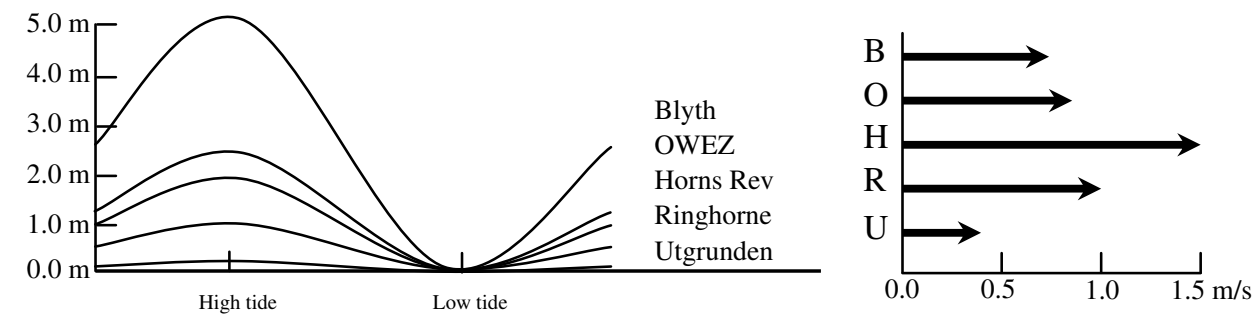

Figure 3.4 Tidal ranges and current velocities for the 5 reference sites

Storm surge is an increase in the water level due a nearby storm system. The storm surge determination is less straightforward than the local current. If site-specific data is available, the storm surge can be extracted from long-term measurements and an extreme value analysis. If no site-specific data is readily available, crude estimates are required, as was the case for Utgrunden. Figure 3.5 shows the established storm surge heights per site.

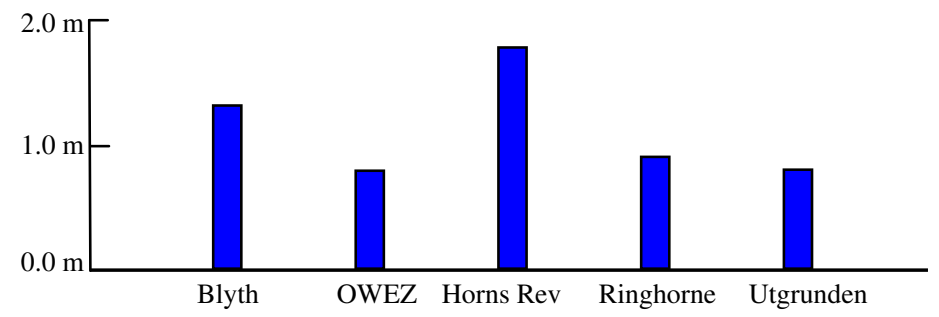

Figure 3.5 Design values for extreme storm surge for the 5 reference sites

\subsubsection{The seabed}

\section{Bathymetry}

The local bathymetry can influence the wave and current parameters significantly. Firstly, sand banks and shallow areas on the seaward side of an offshore wind farm may reduce the maximum wave height within the farm: higher waves will break on the bank. Although large sand banks are usually relatively stationary throughout the 20 year lifetime of an offshore wind farm, the edges of these banks should be avoided for a construction site as they are likely to shift during the lifetime.

Secondly, a mildly sloping seabed may require additional analyses of wave data when the point of measurement lies further offshore. Phenomena like shoaling (waves becoming higher and steeper towards shallower water) and refraction (wave direction turning perpendicular to the coast) may affect the wave climate significantly. 
Thirdly, seabed anomalies within the wind farm can cause breaking waves on offshore wind turbine support structures. This is the case at Blyth, where the turbines are positioned on a rocky outcrop on the seabed, causing fascinating breaking waves.

Finally, the bathymetry throughout the wind farm is unlikely to be uniform and flat. This means that individual turbines are located in deeper or in shallower water; site specific design within the wind farm may become economical when water depth variations are large.

\section{Soil profiles}

The most difficult environmental data to acquire is relevant information of the seabed. As soils can vary dramatically from location to location, even within a wind farm, the best solution would be to hire an expert company to drill holes to determine the soil layering and the soil data at all envisaged turbine locations.

For preliminary design, the soil profiles of nearby structures may be used, should they be available. A geological desktop study can reveal the historical build-up of material from activities in the area: the outflow of rivers giving layers of clay (OWEZ), ice-age glaciers hinting at layers of glacial till and large boulders (Horns Rev).

The soil investigation at the OWEZ site revealed a "mud channel" within the concession area, a small stretch of cohesionless soil. Figure 3.6 shows the channel and the adjusted farm layout with turbines avoiding the softer area.

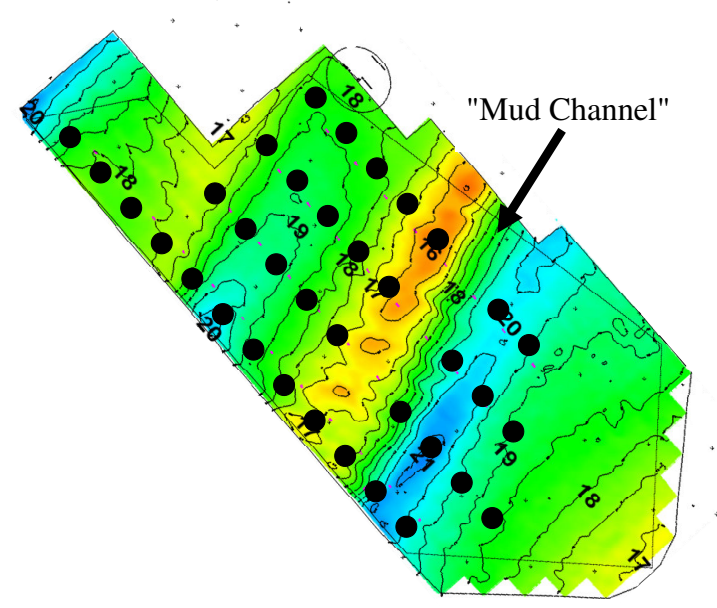

Figure 3.6 Detailed depth chart and general layout for OWEZ with split placement avoiding the "mud channel"

The build-up of the top layer of the soil has another effect on the structure: non cohesive materials like sand may be washed away around the support structure: scour. 
As clay is less susceptible to scour, layers of clay near the surface may limit the depth of the scour process considerably compared to sites with a sand-only top layer.

\section{Moving sand dunes}

A final design driver can be the presence of moving sand waves or sand dunes within the wind farm. These moving masses of sand are part of the large scale morphological system and cannot be counteracted with preventive measures as is possible for a local phenomenon such as scour. Moving sand dunes with an amplitude of $1 \mathrm{~m}$ are found north of the OWEZ site. Sand dunes have an effect on the required foundation depth: all structures must be designed for a local change of the seabed to the extent of the amplitude of the dunes. Furthermore, the cable guiding J-tube needs to be designed in such a way that free-spanning will not cause damage to the cable.

Sand waves can have a significant impact on the design, or at least on the uncertainty in the design, of offshore wind farms, and only recently methods have been developed to establish the presence of moving sand waves. Currently, the most effective method is to use satellite images over a longer period of time to study sand wave movements.

\subsection{Critical design and engineering details}

Although the primary structure will be designed for the ultimate limit state and the fatigue limit state, some critical details need to be assessed before these checks can be carried out. In this section the most important details found in the available documents are presented. For each detail, the main design considerations are summarized. The aim of this section is to give a general overview of relevant details to be considered.

\section{Transition piece}

When monopiles are installed in any type of soil but rock, the pile is usually driven into the ground. In the typical design solution a transition piece is then fitted over the driven pile and connected with grout. The transition piece fulfils several critical roles in the installation process:

- nothing can be connected to the driven foundation pile: pile driving introduces enormous accelerations, damaging or severing anything connected to the pile, especially on the top of the pile

- by connecting appurtenances (J-tube, anodes) to the transition piece, everything can be pre-installed onshore mostly even in workshop conditions

- to a certain extent misalignment of the driven pile can be corrected to make sure the tower will be perfectly vertical

- the transition piece provides a flange to which a "standard" onshore tower can be fitted, if appropriate. 
The main design criterion of the transition piece is the transfer of the vertical and horizontal loads from the tower to the foundation pile. The height of the transition piece is governed by the extreme load cases for the grout

\section{Platform}

The access platform may have a different function from one offshore wind farm to another. At some offshore wind farms, the platform is only meant to carry people and light equipment, at Utgrunden and other locations with GE turbines, the platform is also used to carry a container with the switch gear.

Although the maximum load case will differ for these functions, the method of design is very similar: the required platform area must be supported by steel outriggers which can be supported by diagonal supports connected to the tower or the transition piece. The design loads for the OWEZ site are summarized in table 3.1.

Table 3.1 Design loads for access platform

\begin{tabular}{l|l}
\hline distributed load on platform & $4.0 \mathrm{kN} / \mathrm{m}^{2}$ \\
point load on platform (area: $0.1 \times 0.1 \mathrm{~m})$ & $4.0 \mathrm{kN}$ \\
horizontal line load at top of the handrail & $1.5 \mathrm{kN} / \mathrm{m}$ \\
vertical line load at top of the handrail & $1.5 \mathrm{kN} / \mathrm{m}$
\end{tabular}

The platform deck usually consists of steel grating. The design height of the platform should be such that under no circumstances wave tops can reach the grating. A normal build-up of height above mean sea level is shown in table 3.2, with typical values for OWEZ.

Table 3.2 Defining the design height of the access platform with typical values for OWEZ

\begin{tabular}{l|l|l}
\hline Highest astronomical tide & MSL +1.4 & {$[\mathrm{~m}]$} \\
$3 / 4$ of $H_{\max }$ & $0.75 * 13.99$ & {$[\mathrm{~m}]$} \\
storm surge & 1.0 & {$[\mathrm{~m}]$} \\
air gap & 1.5 & {$[\mathrm{~m}]$}
\end{tabular}

The air gap in table 3.2 is critical for monopiles as the diameter is relatively large and will therefore cause wave run-up. As the grating is usually kept in place by gravity only, upward wave impact may upturn and damage the grating as has been reported for the Blyth turbines, the met-mast at the OWEZ site and a number of turbines at Horns Rev.

\section{J-tube}

The J-tube is a steel or plastic tube exiting the column at the access platform level and extending to the seabed where it bends to become horizontal for pulling the power cable and to guide the cable to the seabed. The J-tube is usually pre-fixed to the transition piece as shown in Figure 3.7. 

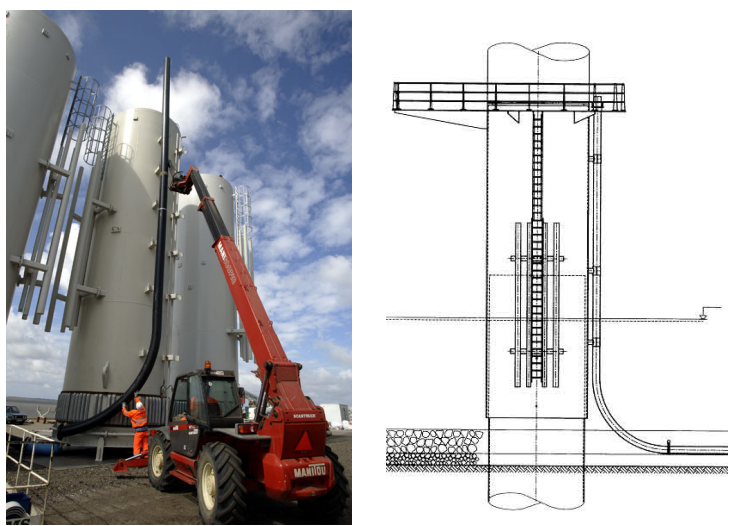

Figure 3.7 Horns Rev transition piece with J-tube

The J-tube presence adds to the horizontal wave loading on the structure and should be taken into account in modelling wave and current loads. An offshore wind turbine at the end of a connection string will only have one tube, whereas most turbines are connected with their neighbours in a string via 2 J-tubes In cases where the power of several strings is gathered at one offshore wind turbine and directly transferred to shore, three or more J-tubes may be required, adding considerable wave loads to the overall structure. More detailed investigation will then be in order as the external tubes will also affect the flow around the monopile, making a re-evaluation of the Morison coefficients necessary.

For the part on the seabed, the J-tube is usually protected with gravel. This will prevent scour and therefore free-spanning of the J-tube and will fix the tube so that no horizontal motions will occur. The exiting cable is usually trenched into the seabed. On a rocky seabed, as at Blyth, the cable needs to be fixed with crams or dumped rock. As this was not done after initial installation, the cable mantle was damaged at the exit point, requiring costly repairs at Blyth.

Currently, new J-tube configurations are introduced allowing the J-tube to follow scour hole profiles and giving the required stability without support of gravel. Another possibility is to guide the cable through the inside of the monopile and let it exit through a pre-cut hole at seabed level. The hole must be reinforced as it introduces a weakness in the support structure at the location where dynamic (fatigue inducing) stresses are almost the highest.

\section{Boat landing}

The boat landing is a contraption of two or more fenders encasing a steel ladder. Like the J-tube, the boat landing is pre-installed on the transition piece. All elements of the boat landing will go through the waterline, adding hydrodynamic loading to the support structure, which should be taken into account in the overall design. Adding more boat 
landing locations, to access the turbine from different directions, will therefore add a significant amount of wave loading.

The wave loads and self weight of the boat landing are less governing than the impact of the maintenance vessels. The design philosophy then becomes to make sure the boat landing will deform under extreme ship impact to prevent damage to the main structure. Details on ship impact are treated next.

\section{Vessel impact}

The philosophy behind vessel impact assessment is the following:

- accidental impact by maintenance vessels should be absorbed by plastic deformation of the boat landing fenders

- accidental impact by maintenance vessels on other parts of the structure should not exceed the plastic deformation limit of the structure

- the probability of accidental impact by larger vessels or containers, and the effects on the structure, should be assessed, but protective measures may not be economically feasible.

The Germanischer Lloyd guideline [20] gives a calculation method to assess the total external load from ship impact on the structure using:

$$
\begin{gathered}
F_{s i}=P_{0, s i} \\
F_{s i}=v_{s i} \sqrt{c_{s i} a_{s i} m_{s i}}
\end{gathered}
$$

with:

\begin{tabular}{l|l|l}
\hline$F_{s i}$ & Impact load on structure as horizontal line load over the width of the foundation & {$[\mathrm{N}]$} \\
$P_{0, s i}$ & minimum crushing strength of the impacting part of the vessel & {$[\mathrm{N}]$} \\
$v_{s i}$ & velocity of impact vessel (at least $0.5 \mathrm{~m} / \mathrm{s})$ & {$[\mathrm{m} / \mathrm{s}]$} \\
$c_{s i}$ & stiffness of impacting part of the vessel & {$[\mathrm{N} / \mathrm{m}]$} \\
$a_{s i}$ & added mass coefficient $1.4-1.6$ side ways, 1.1 bow or stern collision & {$[-]$} \\
$m_{s i}$ & displacement mass of impact vessel & {$[\mathrm{kg}]$}
\end{tabular}

Should this level of detail not be available, GL recommends an impact load of $5 \mathrm{MN}$.

\section{Scour}

Scour is the name for the erosion of loose seabed material directly around offshore structures. The resulting local lowering of the seabed has three consequences for the support structure design:

- reduction of penetration depth, possibly requiring the pile to be driven deeper

- reduction of foundation stiffness, affecting the structure's natural frequency

- possible free-spanning of the J-tube and cable. 
A review of the available theory and measurements [63] revealed that design requirements prescribe the expected scour depth to pile diameter ratio to be between $1 D$ and $2.5 D$, while measurements of real structures point into the direction of $1 D$ to a maximum of $1.3 D$. The effects of scour will also be treated in chapter 7 .

To protect the support structure against scour, a gravel bed is usually dumped around the structure. The mechanism driving scour is that the water particle velocity due to current and waves is increased close to the structure as the current presses around it. This increased water flow is able to stir up and transport small particles, creating scour. By dumping larger particles, gravel or rock, the seabed remains stationary. The design of the rock size is therefore driven by the expected current velocity. Should larger rocks be required, a filter layer of smaller gravel is applied to prevent sand particles from being washed from underneath the rock layer.

\section{Corrosion protection}

The steel support structure is susceptible to corrosion. Four methods of corrosion protection can be applied:

- add extra wall thickness (a corrosion allowance) to have enough reserve to compensate steel consumption throughout the lifetime;

- painting, used above water and in areas sometimes submerged;

- impressed current, active electrical current preventing electrochemical process of corrosion in continuously submerged steel;

- anodes, sacrificial material creating a electrochemical process to prevent corrosion in continuously submerged steel.

Of the above only anodes result in extra loading on the total structure as they add submerged volume. However, the anodes are usually fitted near the seabed where wave action has only minor effect.

\section{Marine growth}

Structures in the sea will be covered with marine growth soon after installation. This marine growth increases the dimensions and the total submerged volume, adding extra wave loading to the structure. Furthermore, the roughness is increased requiring reconsideration of the drag coefficient in the Morison equation. The general assumption for marine growth for design in the southern North Sea is given in table 3.3.

Table 3.3 Marine growth in the southern North Sea [19]

\begin{tabular}{c|c}
0 to $-10 \mathrm{~m}$ MSL & $50 \mathrm{~mm}$ \\
-10 to $-20 \mathrm{~m}$ MSL & $40 \mathrm{~mm}$
\end{tabular}

Some types of paint have the ability to prevent marine growth from occurring. Usually, these paints are only applied in areas where marine growth hampers day to 
day operation, for instance on the ladder. Reports from Blyth show that the ladder mostly needs to be cleaned before accessing the turbine. Figure 3.8 shows a picture of the met-mast at the OWEZ site, during installation and one year after installation.
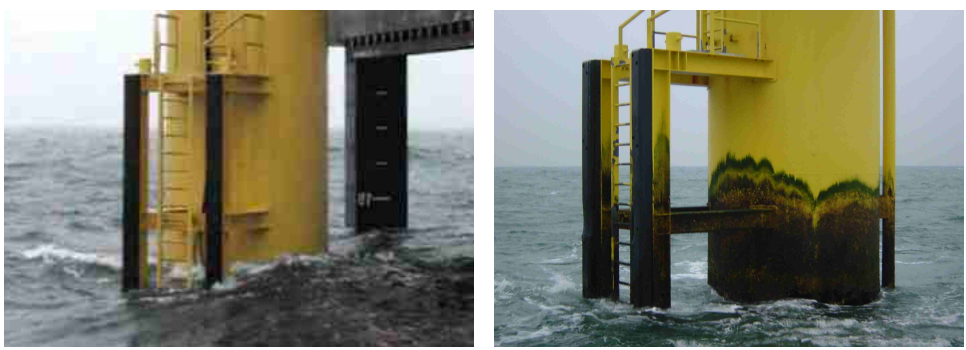

Figure 3.8 Marine growth at OWEZ met-mast, one year after installation

\subsection{Ultimate limit state checks}

\subsubsection{General considerations}

The general approach to test whether a structural design is capable of withstanding the maximum design loads is to analyse all external loads, define relevant design cases, apply these loads on the design and check whether the structure meets the ultimate limit state requirements, i.e. meets or fails the check. This basic procedure is depicted in Figure 3.9.

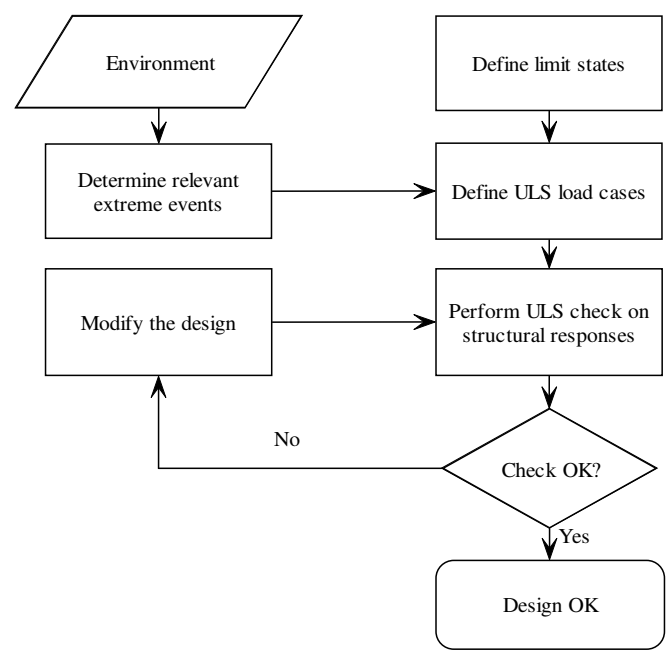

Figure 3.9 Basic extreme load check flowchart 
When the structure fails under a certain load, the initial structure must be re-designed to pass the load case in the next check. When the structure amply passes all load cases, the design can generally be optimized by reducing material.

\section{Probability considerations}

All structures need to be tested for a large number of load cases. A load case generally consists of a combination of different external loads, which are not necessarily all at their maximum at the same time. Therefore a load case inherently corresponds with a certain probability level. As an alternative to defining specific (deterministic) load cases, probabilistic design methods can in principle be applied (see 3.5.4), but this is presently still outside the design practices.

\section{Incorporating dynamics}

As noted, load cases for ultimate limit state checks are usually applied as deterministic loads. However, dynamics may be an important factor when assessing the response of the structure. To take this into account, an estimate of the dynamic amplification factor (DAF) can be established by running a specific load case through a dynamic model and dividing the dynamic response by the corresponding static response. The DAF can then be used as a multiplication factor to incorporate dynamics, after which the load case calculation can be treated in a quasi-static manner. It should be noted explicitly that such simplified treatment of dynamics by means of a DAF is only acceptable if dynamic response is assessed in the stiffness controlled region, so that the dynamic and quasistatic contributions to the overall response are approximately in phase. For extreme response in the resonant or inertia dominated regions, more advanced dynamic analysis is required.

\subsubsection{Design load cases for offshore oil \& gas platforms}

To illustrate the generic procedure in the previous section, the design of the Ringhorne structure is used. This structure was designed, constructed and installed by Heerema during 2000-2002 for ExxonMobil. The environmental conditions for the extreme design load case can be summarized as shown in Figure 3.10. All environmental loads are assumed to act simultaneously in one big storm: maximum wind, maximum wave height and maximum tidal current. These are all contributing to the maximum stress in the members, which should not exceed the design values for all members. 


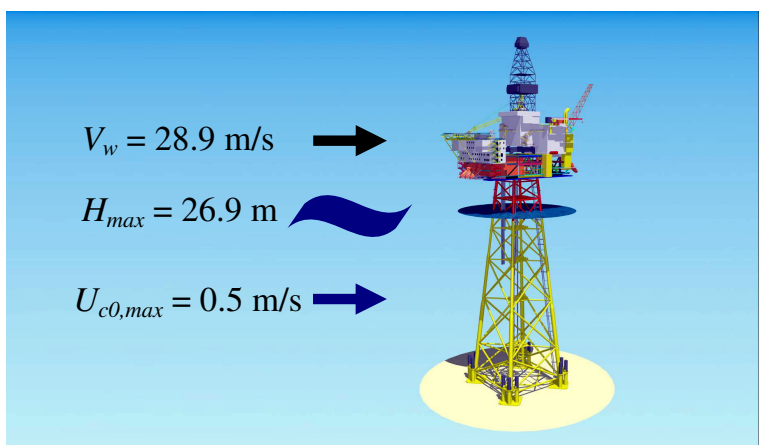

Figure 3.10 Schematic design wind, wave and current conditions for the ultimate limit state

Of course, this one case is not the only check. All imaginable load combinations that could lead to a maximum response anywhere in the structure are checked: loads that occur during service, installation, collision, earthquake, drilling activities. Some loads can be critical for certain parts of the structure, where other, bigger loads are not.

\subsubsection{Offshore wind turbine, extreme load design}

\section{Horns Rev}

At Horns Rev, the design documents are very straightforward in the maximum design loads for the support structure: all loads are determined based on rule-of-thumb estimates of maximum waves, wind and currents. The apparent philosophy behind this approach is that the shallow depth at the site will limit the maximum wave height to the breaking wave height. The RNA loads are only given as shear force and overturning moment at $2.5 \mathrm{~m}$ above mean sea level (MSL), as shown in Figure 3.11. No reference to the origin of the RNA loads is given.

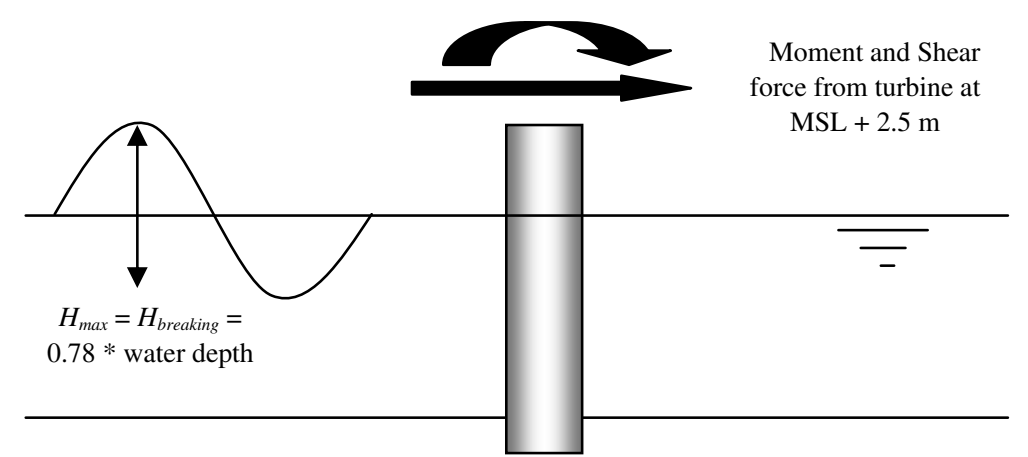

Figure 3.11 Maximum design load case on the support structure of the Horns Rev turbines 


\section{Utgrunden}

At Utgrunden an attempt was made to determine both the wave scatter diagram and the maximum wave probability from assumed statistical distribution of wave heights. The maximum wave height was further limited by the breaking wave height in a water depth of $10 \mathrm{~m}+0.8 \mathrm{~m}$ surge and tide. In the report a preliminary extreme load case check has been performed for a number of cases as prescribed by Germanischer Lloyd. These load cases are shown in table 3.4.

Table 3.4 Extreme load cases checked for Utgrunden

\begin{tabular}{l|l|l|l} 
ID & Load case & wind & wave \\
\hline \hline M1.1 & Maintenance, annual gust & $1 \mathrm{yr}$ gust & $1 \mathrm{yr}$ reduced \\
E1.1 & Extreme operating gust, reduced wave & $V_{\text {cut-out }}+V_{\text {gust }}$ & $50 \mathrm{yr}$ reduced \\
E2.1 & Extreme gust, reduced wave & extreme 5 gust & $50 \mathrm{yr}$ reduced \\
E2.2si & Extreme single wave, reduced gust & extreme 1 min gust & extreme wave \\
E2.2st & Extreme sea state, reduced gust & extreme 1 min gust & extreme sea state \\
E2.4 & Extreme sea ice & extreme 10 min gust & -
\end{tabular}

\section{Blyth}

At Blyth breaking waves do also occur. As at Horns Rev, this makes determining the maximum wave height easy: never larger than the breaking wave height. But in this case the support structure design did not benefit. Due to the very location, the waves would also most certainly break against the support structures, as was registered by the camera mounted on the northern turbine to monitor this phenomenon, shown in Figure 3.12 .

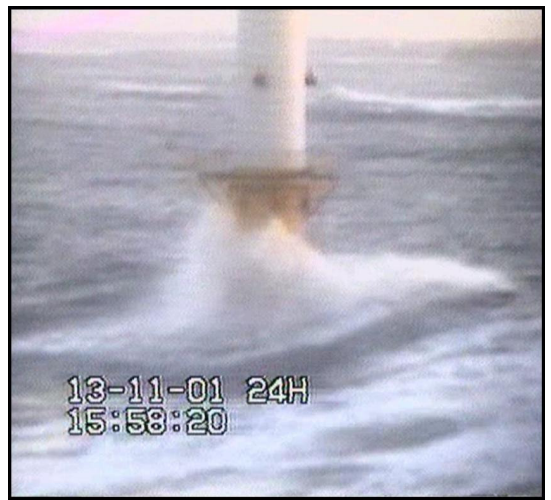

Figure 3.12 Video snapshot of breaking wave on southern turbine at Blyth 
For this reason, the design document includes an extensive study on the subject. Additional, detailed data of the environment was available through a site study by Fugro-Geos based on the NESS-NEXT database. Because the NESS-NEXT data point available near Blyth was still $20 \mathrm{~km}$ removed from the site and valid for a water depth of $40 \mathrm{~m}$, a bathymetry model was used to transform the offshore data to the shallow water site. The model outputs were checked for two measurement locations in the vicinity of Blyth.

\section{OWEZ}

The Near Shore Wind farm site has been the subject of several studies since the early nineties. Table 3.5 shows three different values for the extreme wave height, taken from different sources. The highest value in this table was eventually selected for the design.

Table 3.5 Extreme wave design values from 3 different sources for the OWEZ site

\begin{tabular}{l|c}
\hline OPTI-Owecs (NESS) & $12.8 \mathrm{~m}$ \\
Argoss (Satellite) & $13.2 \mathrm{~m}$ \\
Buoy (RIKZ) & $13.99 \mathrm{~m}$
\end{tabular}

\subsubsection{Full probabilistic method to find extreme response}

The current design practices as described in the previous sections all focus on predetermining the extreme external event and calculating the structure's response to that event. This approach only works when all extreme responses are instigated by extreme external events. For offshore wind turbines this will not always be the case. A simple fact already points in that direction: the maximum turbine thrust in a pitch regulated machine occurs at rated wind speed. The turbine is at that instance trying to capture maximum power from the wind speed. A higher wind speed will result in pitching of the blades and "bleeding" of energy to prevent the generator from overloading. So, nonextreme external events can cause the maximum structural response.

To explore this phenomenon Cheng [43] ran a large number of simulations and used several extreme value calculation methods to find the most probable extreme response. The approach first selects the critical operational windows of the offshore wind turbine for which extreme responses can be expected. For these windows, the upper tail of the environmental conditions related to the window are used for dynamic time domain simulations. By filtering the peaks of the response signal and applying a curve fitting technique, the probabilistic maximum response is found. A flowchart of the method is given in Figure 3.13.

The method is currently an Annex to the draft IEC 61400-3 standard as an optional analysis. The main bottleneck is the rather detailed model required for the analysis and the manner in which the critical operational windows have to be established, encircled in Figure 3.13. 


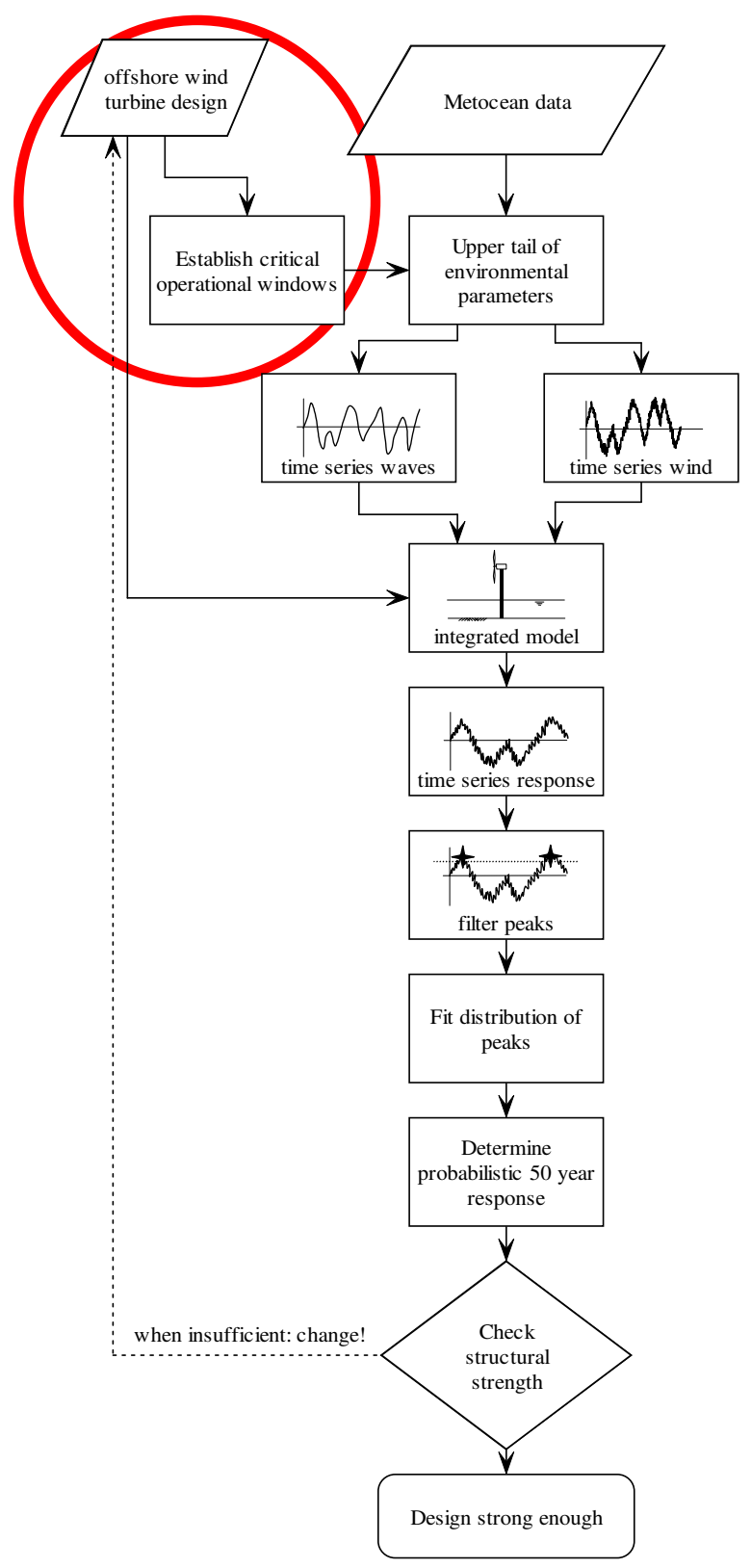

Figure 3.13 Extreme response based approach [43] 


\subsubsection{Conclusions on ultimate limit state checks}

From the previous sections it can be concluded that the current practice generally works well. The design can be easily changed to meet the required strength criteria. There is a general trend towards full probabilistic design focussing on extreme response, rather than on extreme external loads. For offshore wind turbines in particular this probabilistic method can improve the design, as has already been demonstrated in theoretical studies. This thesis will not go deeper into ultimate limit state theories, however.

\subsection{Fatigue checks}

\subsubsection{Introduction}

The fundamentals of fatigue have been introduced in section 2.8. This section will further detail the application of fatigue calculations as used in the design documents available for this thesis. The fatigue calculation method used for the Ringhorne structure is the standard method used throughout the offshore industry. Section 3.6.3 shows that methods differ from project to project for offshore wind turbines. Conclusions on how to proceed on offshore wind turbine fatigue are summarized in section 3.8.

\subsubsection{Fatigue of the Ringhorne jacket}

Although the common belief in the wind turbine community is that offshore structures are designed so stiff that no dynamic response occurs and therefore fatigue is not really a design driver for fixed offshore structures, reality is different. The natural frequency of the Ringhorne jacket was found to be $0.25 \mathrm{~Hz}$, making it susceptible to wave induced dynamic response as shown in the frequency plot in Figure 3.14.

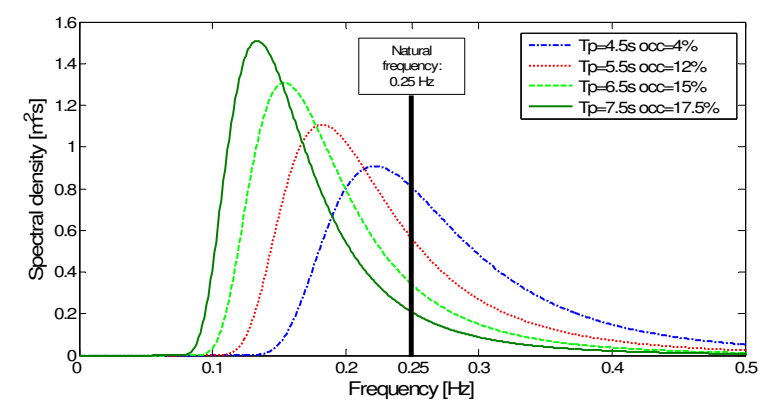

Figure 3.14 Wave spectra and frequency of occurrence for 50\% of all sea states at the Ringhorne location; first natural frequency at $0.25 \mathrm{~Hz}$ receives high loads, resulting in resonant behaviour 
For the site, a detailed wave scatter diagram was available. With the jacket modelled in the finite element frequency domain program SACS, fatigue checks can be done rather quickly. Because the structure consists of a large number of joints, every joint needs to be checked for fatigue resistance. Figure 3.15 shows the flowchart for filtering the critical joints, establishing the required stress concentration factor (SCF) for every particular joint and, if needed, optimising the joint dimensions to make it pass the fatigue check. Joints are a connection of several tubulars and the stress differs per radial location on every tubular (12-3-6-9 'o clock orientations) as well as on the brace side and the chord side of the connecting weld. All these locations are checked for the critical joints (not depicted in Figure 3.15)

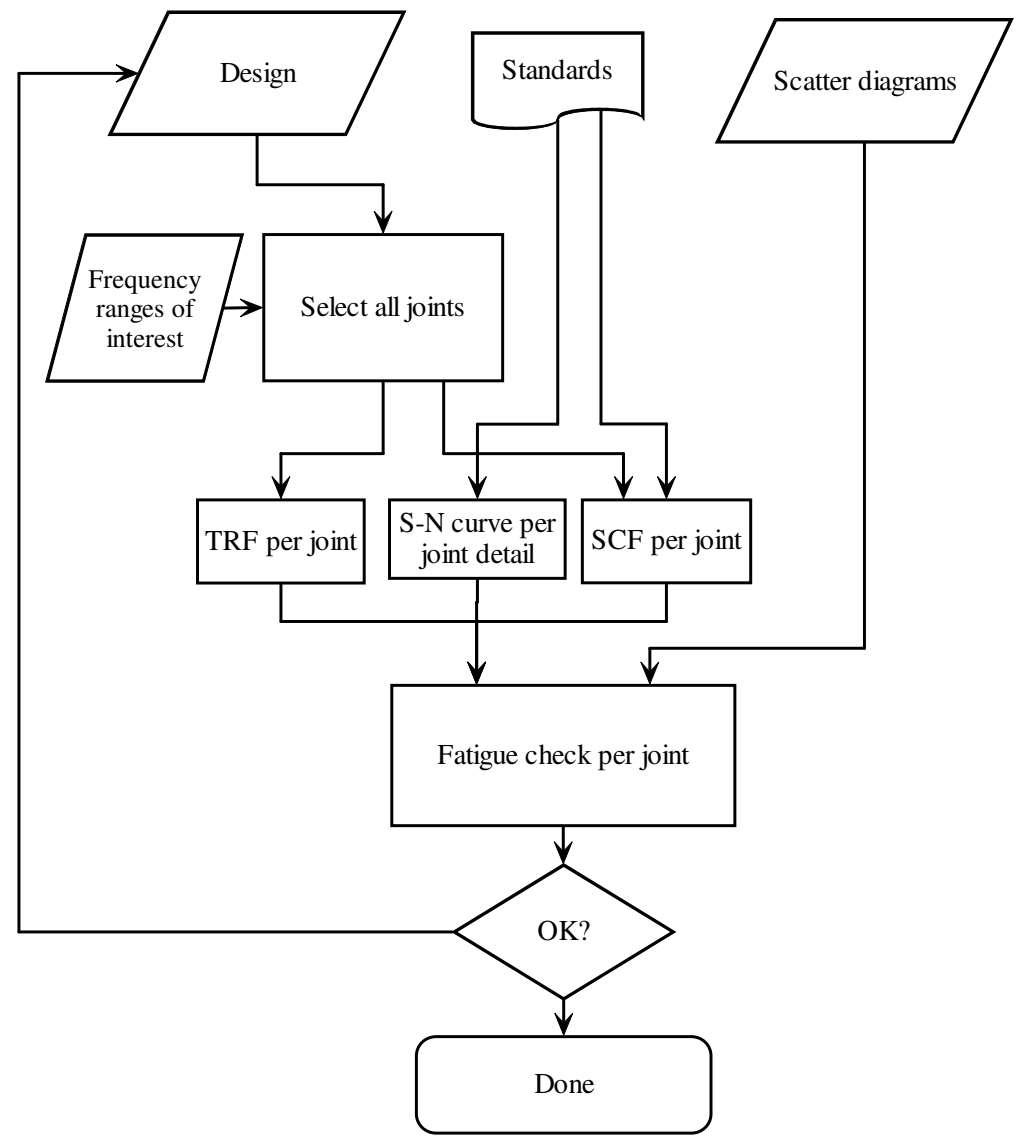

Figure 3.15 Flowchart for fatigue check of joints in the frequency domain (Ringhorne) 


\subsubsection{Offshore wind turbine fatigue analysis: a variety of methods}

\section{Utgrunden}

The methods as documented in the Utgrunden report were mainly based on knowledge gathered in the Opti-OWECS study [5] and [4]. The basics of this design approach are summarized here. The turbine manufacturer did a full lifetime fatigue analysis in the time domain for the wind loads on the rotor. For the hydrodynamic loads, a frequency domain model has been used. To incorporate the aerodynamic damping on the structure's response when the turbine is operating, two models were used: low damping $(1.5 \%)$ in case the turbine is not operating and high damping $(5 \%)$ for production load cases. The combined fatigue damage was calculated by quadratic superposition of the separate wind and wave fatigue contributions. The approach is depicted in Figure 3.16.

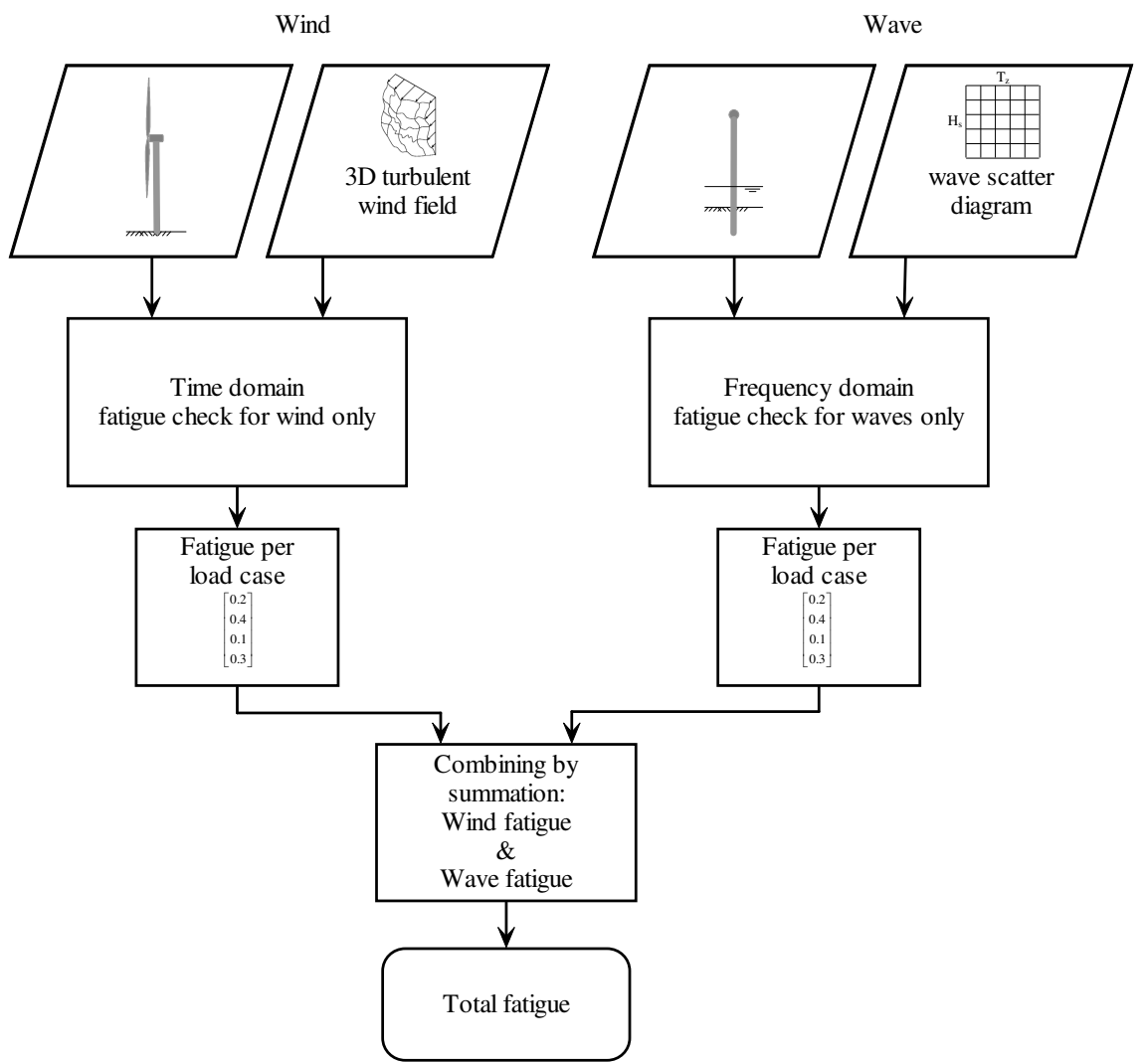

Figure 3.16 Combining the results of separate fatigue analyses for wind and wave loads (Utgrunden) 


\section{Blyth}

For Blyth a fully integrated model of the turbine and support structure was available to the design team. The metocean study [56] had resulted in a load case table consisting of 90 cases of wind speed, significant wave height, mean zero-crossing wave period and wind and wave direction. The flowchart in Figure 3.17 shows the method of deriving the wind-wave load case table.

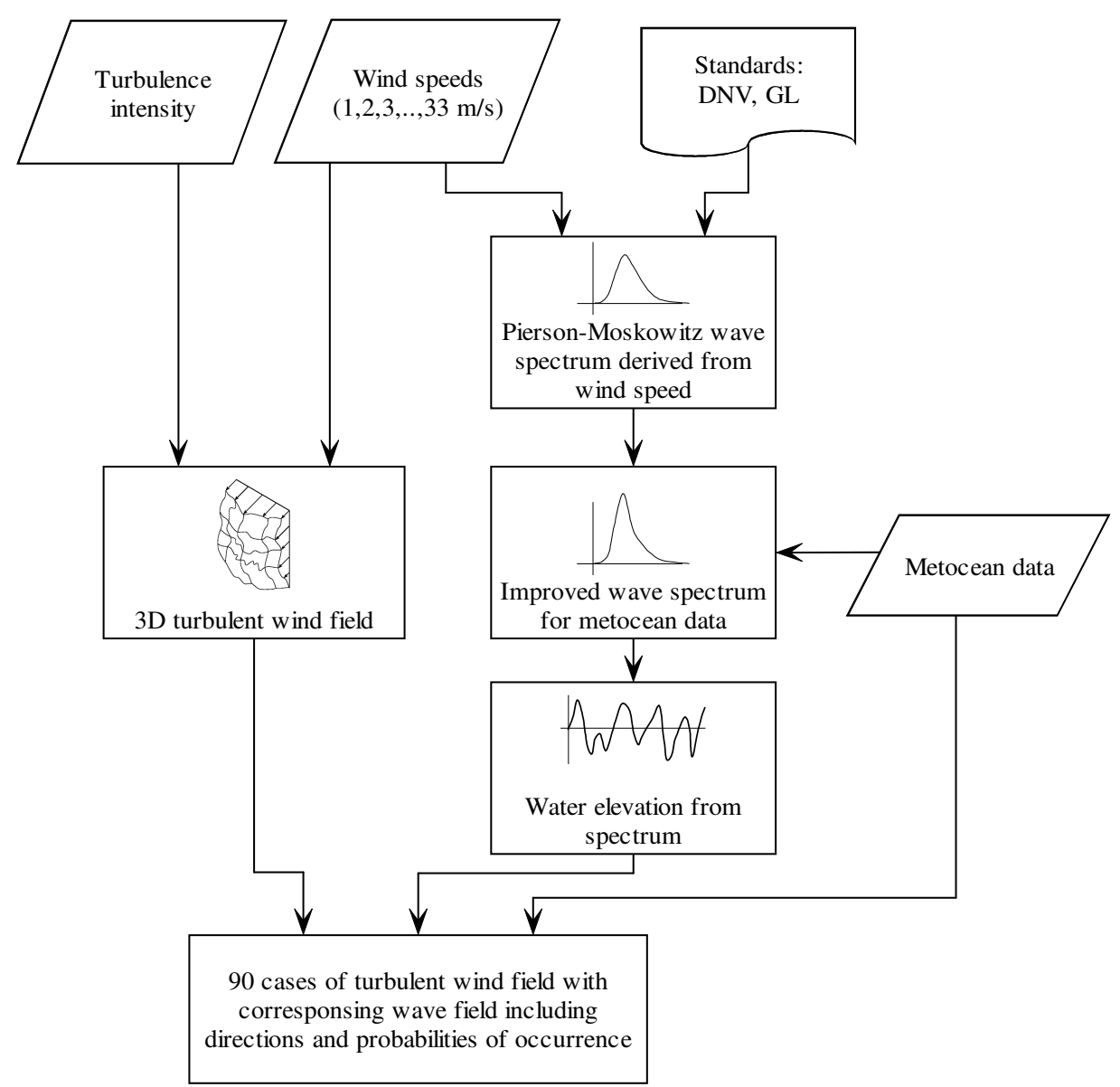

Figure 3.17 Processing the metocean data to 90 wind and wave directional states (Blyth)

The load cases were simulated in Bladed with a single time series of 20 minutes in the time domain for each combination; different random seeds were used for the wind and wave fields for each run shown in Figure 3.18. In the design report a comparison of the load cases was made, acquired through the rather complex processing method depicted 
in Figure 3.18 and directly from the NESS-NEXT report; the comparison shows that the processing method delivered significantly more severe states for the turbines. The report states that the analysis was therefore conservative.

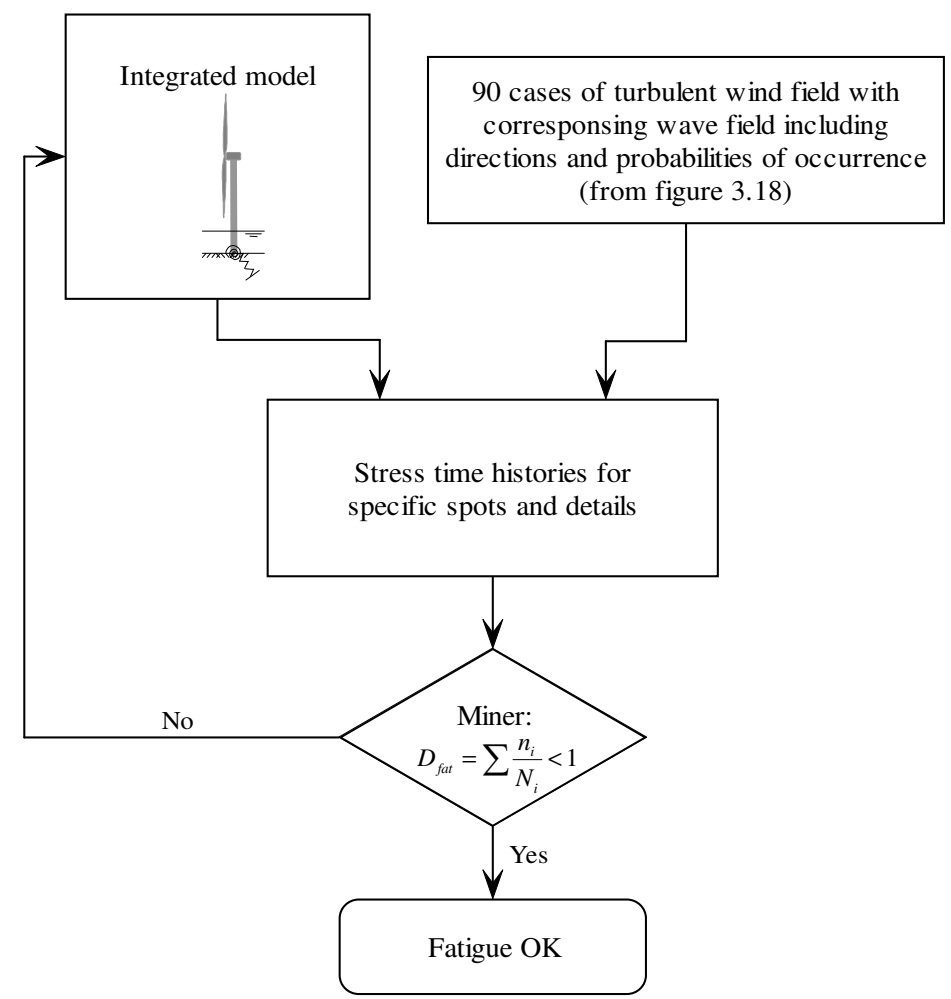

Figure 3.18 Fatigue calculation for 90 load cases (Blyth)

\section{Horns Rev}

In the Horns Rev design report fatigue was only treated very superficially. The philosophy was to assess fatigue based on the maximum amplitude of the stress in the support structure due to dynamic wind loading. Waves were assumed to introduce very low stresses and were therefore neglected. The maximum stress variation amplitude was found to be lower than the fatigue threshold of the $S-N$ curve used. Fatigue was therefore neglected entirely: "no fatigue damage will be present".

\section{OWEZ}

As the process of awarding the contract to the selected EPC (engineering, procurement and construction) contractor was rather lengthy, no legal framework existed between the 
partners in the OWEZ project in the first years which would have facilitated proper integrated design. As the turbine manufacturer was reluctant to share detailed turbine information and the offshore contractor did not have full knowledge of integrated offshore wind turbine design, a rather complicated design method emerged.

Figure 3.19 shows the flowchart of the design steps between the turbine manufacturer and the offshore contractor. The basic design of the foundation pile is done by the offshore contractor and been made to fit the large diameter bottom end of the predesigned tower. Then the design is transferred to the wind turbine manufacturer to combine support structure and turbine and perform fatigue calculations proposed by the offshore contractor. Finally, the offshore contractor incorporates the fatigue calculation results in his documents and takes responsibility for the outcome.

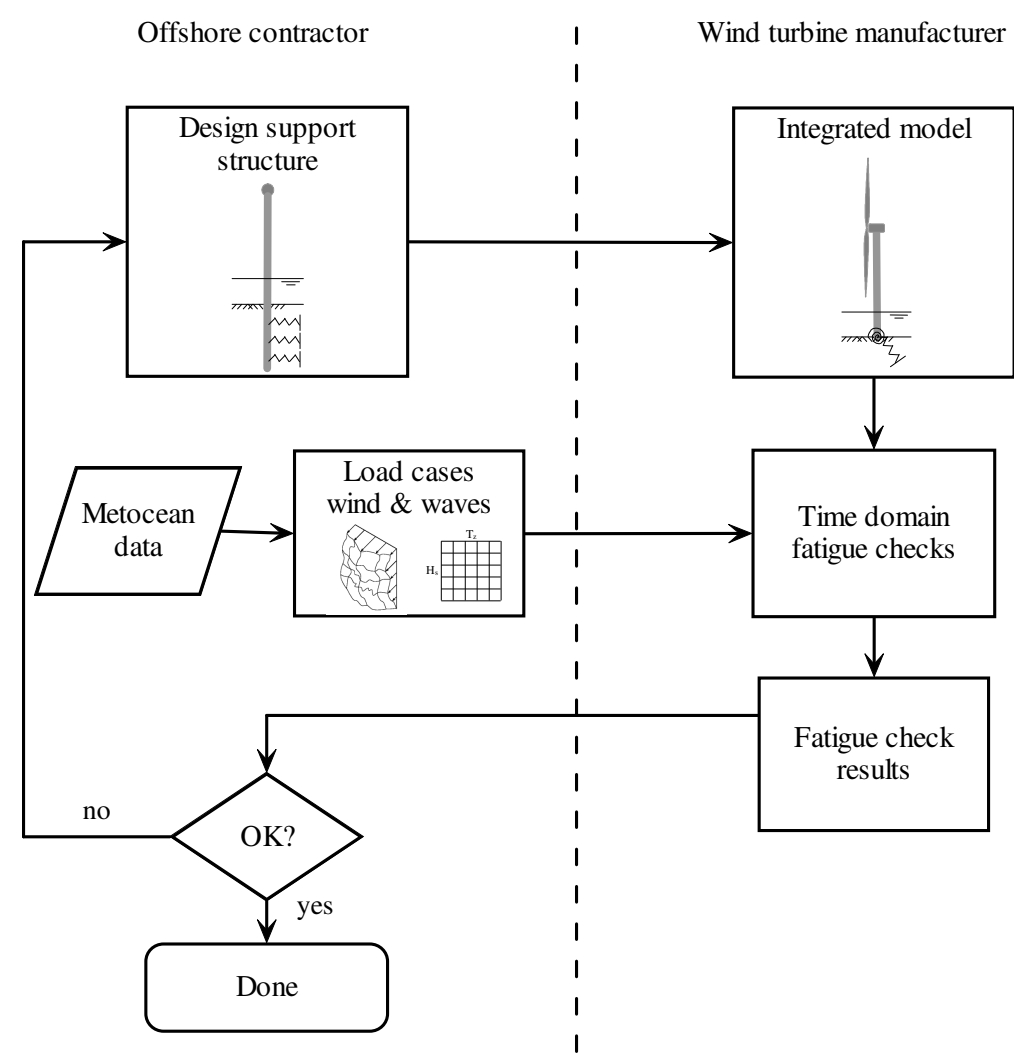

Figure 3.19 Flowchart of how the turbine manufacturer and the offshore contractor shared the design process (OWEZ) 


\subsection{More options for fatigue: frequency domain}

For the executed projects, the previous section clearly shows no similarity in assessing the fatigue damage of offshore wind turbine support structures. With today's abundance of computer power, the current method is to run a large number of load cases through complex time domain models. In the early years of wind turbine design, frequency domain methods were developed to make the best of the much more limited computer capabilities. These methods attracted a lot of attention through the 1980s and early 1990s. The method enabled designers to incorporate stochastic properties of the turbulent wind field in the design in a straightforward and transparent way. Although time domain programs were also available, creating large three-dimensional turbulent wind fields required large amounts of computer memory and fast processors, both not available at that time. The arrival of faster computers with more memory enabled modelling of the non-linearities in aerodynamics and control in the time domain, which was the main reason to abandon frequency domain calculations altogether: no commercial turbine design package in the frequency domain is available at this moment. Bigger and better time domain simulation programs offer designers endless modelling options, but are never quite able to give the transparency of the frequency domain. As a participant in the discussion at a recent workshop stated: "Time domain people are able to model all in minute detail, claiming to solve everything; frequency domain people just solve the problem and always seem to have better understanding." [44]

Two mainstream frequency domain programs will be briefly discussed here: Turbload, by Garrad Hassan and Turbu by ECN. The first program was used for the 1990 study of offshore wind energy potential in the EU [26], but abandoned not long after in favour of further development of the time domain program Bladed. Turbu on the other hand has been under construction throughout the nineties to upgrade it to a full offshore wind turbine simulation tool. Below, the description of Turbu I is used [45].

Both programs are based on the same principles. For a period of 10 minutes, the stochastic properties of the wind field are considered to be stationary, but also the turbine responses can be assumed to be stationary: blade pitch settings, induction factor and rotational speed. At the time when both programs were used, this was very true because most turbines were fixed speed. To solve the non-linear blade-elementmomentum calculation for this presumed steady state, the assistance of a time domain program was required. With steady state solutions per wind speed range, the frequency domain program could proceed.

The main feature of the frequency domain approach was to transform the wind spectrum as seen by a stationary observer to a rotational wind spectrum as seen by the blade. This process is described in section 2.6. By applying the rotationally sampled spectrum to the transfer function for wind speed to a particular blade or tower response, 
a response spectrum was acquired. The response spectrum of the bending stress could then be post-processed to, for instance, rainflow ranges to facilitate fatigue calculation. For this post-processing two options were given: recreation of time series from the response spectrum or direct calculation through spectral parameters. Figure 3.20 shows the flowchart of Turbload from [26].

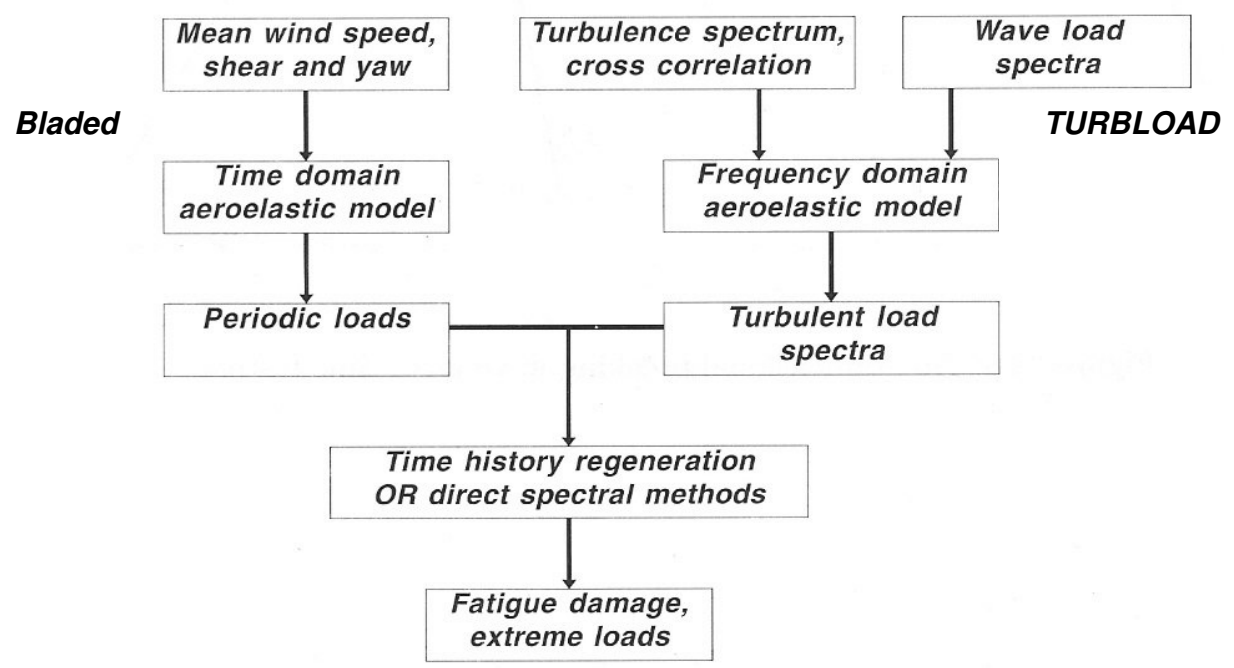

Figure 3.20 Flowchart of Turbload calculation process [26]

The flowchart in Figure 3.20 shows that the execution of Turbload uses time domain calculation of periodic loads in Bladed (the left side). For every mean wind speed a simulation in Bladed is required to solve the blade-element-momentum calculation, incorporating non-linear turbine features.

The working of Turbu is very similar and uses input from the time domain program Phatas. Table 3.6 shows the input parameters that Turbu requires as well as the parameters retrieved from Phatas. 
Table 3.6 Input of Turbu, directly and via Phatas [45]

\begin{tabular}{|c|c|c|c|}
\hline \multicolumn{3}{|c|}{ TURBU-I } & \multirow{2}{*}{$\begin{array}{c}\text { Phatas } \\
\text { Aerodynamics per } \\
\text { blade element }\end{array}$} \\
\hline Turbine & Wind field & Simulation settings & \\
\hline number of blades & hub height & $\begin{array}{c}\text { inverse of frequency } \\
\text { step }\end{array}$ & radius \\
\hline pitch angle & roughness length & $\begin{array}{c}\text { number of mesh } \\
\text { angles }\end{array}$ & twist angle \\
\hline rotor speed & $\begin{array}{l}\text { exponential decay } \\
\text { constant for } \\
\text { coherence function }\end{array}$ & $\begin{array}{l}\text { number of harmonics } \\
\text { in power spectrum }\end{array}$ & angle of attack \\
\hline hub radius & $\begin{array}{l}\text { lower bound wind } \\
\text { class }\end{array}$ & $\begin{array}{c}\text { number of blade } \\
\text { elements }\end{array}$ & load due to lift \\
\hline rotor radius & $\begin{array}{l}\text { upper bound wind } \\
\text { class }\end{array}$ & output options & load due to drag \\
\hline wake state & & & \\
\hline
\end{tabular}

Although several time domain calculations are required to make both programs work, the length of these simulations can be rather short: only a steady state response needs to be reached.

The programs were validated [26][46] and the speed of calculation was always praised whereas the limitations of linearization were emphasized. Both programs were primarily developed from the turbine component load modelling perspective: blade flap response, drive train torque and only marginally for support structure design.

Recent developments of the TURBU code have resulted in a new version: TURBU Offshore [60]. The code has been completely rewritten in Matlab and hydrodynamic loading capabilities were added. The focus of the program, however, remained the correct representation of aerodynamic interaction and turbine component loading. In a large pan-European project, STABCON [61], aero-elastic instabilities were studied and the new TURBU Offshore code was found to perform very well: aero-elastic instabilities were predicted rapidly and correctly, again proving the usefulness of frequency domain output.

In the description of the new code no real emphasis is put on hydrodynamic modelling, although wave spectra can be used as input and will result in increased response of the modelled structure. As yet, no real effort has been made for thorough testing of the modelling of hydrodynamics.

Because the focus of TURBU Offshore is more turbine related than support structure related, and since the Turbload code has not been used for a decade, these programs will not be studied further in this thesis. 


\subsection{Conclusions on fatigue analysis methods}

The variety of fatigue calculation methods presented in the previous sections shows that there is no single approach being used. The main reason for this seems to be practical and contractual: the design of the foundation pile and transition piece is the responsibility of the offshore contractor, while the tower and rotor-nacelle assembly are the domain of the wind turbine manufacturer. Although this division may seem practical from a contractual point of view, the structure does not feel this very interface and will act dynamically from blade tip to foundation pile.

A way to take this hurdle would be to enforce information sharing between the two parties to make sure a complete model can be created comprising all necessary details. Unfortunately, the turbine manufacturer is usually reluctant to share turbine details and frequently only shares typical bits of information such as "damage equivalent loads", a summary of his fatigue analysis procedure, which can only be used for estimating purposes. On the other hand, if turbine details were to be provided to the offshore contractor, the latter will usually not have the required expertise to use it properly.

Another way would be to create a new method for fatigue assessment of the support structure in the frequency domain. This would enable the offshore contractor to use all his traditional methods, software and experience, and enable him to optimise the support structure. It would even make location specific designs within the wind farm feasible. Furthermore, the frequency domain offers the great advantage of providing the offshore designer with clear information on environmental and structural properties to further speedup the design process.

To make such a method work, the turbine properties need to be linearized for steady state conditions and transferred to the frequency domain. The effect of this linearization needs to be checked carefully to prevent loss of accuracy. By devising a standard method of linearization, the transfer of relevant information from the turbine manufacturer to the offshore contractor can be streamlined without the manufacturer having to share sensitive information.

It is recognised that next to the operational and idling load cases also start-up and stoppages as well as the pile driving activities contribute to the total fatigue damage. As these cases can be analysed separately and added to the total fatigue damage, they are not considered in this thesis.

The frequency domain method for calculating fatigue damage will be described in the following chapters. First, chapter 4 presents the current practice in offshore engineering for fatigue assessment of support structures due to wave loads in the frequency domain. Then, chapter 5 describes an analogous approach for wind loads in the frequency domain, which will be applied in chapter 6 where the method is validated for the support structures of the Blyth turbines. In chapter 7 the method will be applied to the 
design of the OWEZ offshore wind turbine and the effectiveness of the method will be demonstrated for quick parameter variation. 


\section{Frequency domain fatigue due to waves}

\subsection{Introduction}

Chapter 3 concluded that, in the offshore industry, current practice for fatigue assessment relies on frequency domain calculations. The major advantages of this method are that it is very fast, allows quick optimisation and is very transparent: the response spectra are readily understood and can be easily interpreted to further enhance design optimisation.

This chapter adresses the general aspects of frequency domain calculations. First, a linear one degree-of-freedom system is analysed to explore the basics of dynamics and the frequency domain method, including links with time domain simulation. In section 4.3 the technique is applied to an offshore wind turbine support structure by way of example. Then, in section 4.4, linearization of the non-linear wave loads is described. Finally, in section 4.5, the response spectrum for wave induced bending stress is determined and used to calculate fatigue damage of the support structure.

\subsection{Basics of frequency domain calculations: the 1 DOF system}

The most elementary representation of a system is shown in Figure 4.1. The system is subject to an external input and produces an output.

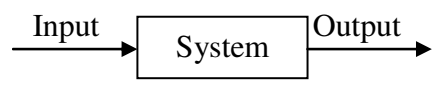

Figure 4.1 Simple system representation: an external input is processed by the system and transformed into a particular output

If the system is taken to be a mass-spring-damper with harmonically varying external load as input and the displacement of the mass as output, Figure 4.2 is created; this figure is identical to Figure 2.41. The system has only one degree of freedom (DOF): the displacement $x$.

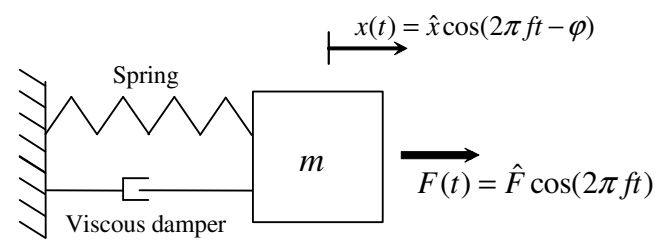

Figure 4.2 Mass-spring-damper system with input load $F$ and output displacement $x$ 
The behaviour of the system can be described by the well known equation of motion:

with:

$$
m \ddot{x}+c \dot{x}+k x=F
$$

\begin{tabular}{l|l|c}
\hline$m$ & mass & {$[\mathrm{kg}]$} \\
$c$ & damping coefficient & {$[\mathrm{Ns} / \mathrm{m}]$} \\
$k$ & spring stiffness & {$[\mathrm{N} / \mathrm{m}]$} \\
$F$ & external load & {$[\mathrm{N}]$} \\
$x$ & displacement & {$[\mathrm{m}]$} \\
$\dot{x}$ & velocity & {$[\mathrm{m} / \mathrm{s}]$} \\
$\ddot{x}$ & acceleration & {$\left[\mathrm{m} / \mathrm{s}^{2}\right]$}
\end{tabular}

The general formulation of equation (4.1) can be programmed in any computational package. The program can then solve the equation of motion for any given time series of input load $F(t)$. Should, for instance, $F(t)=\hat{F} \cos (2 \pi f t)$, the response, $x(t)$, can be found. Figure 4.3 shows 3 different input signals and the system's output.
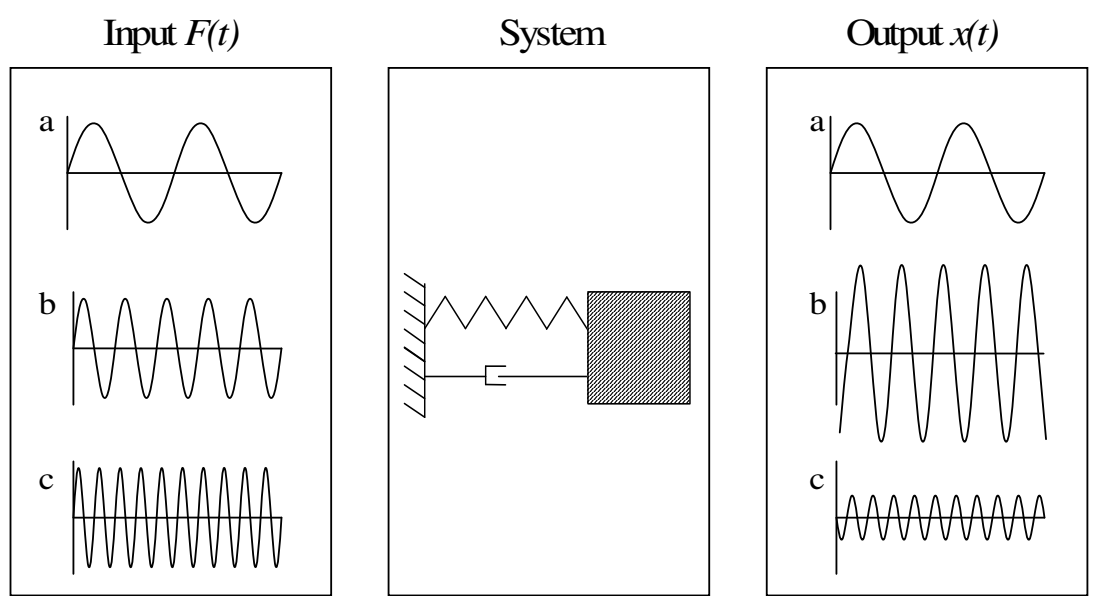

Figure 4.3 Three sinusoidal input signals and the resulting displacement output

By dividing the steady state output amplitude at every input frequency by the corresponding input amplitude, the system's transfer function can be determined, the classic shape of which is shown in Figure 4.4. 


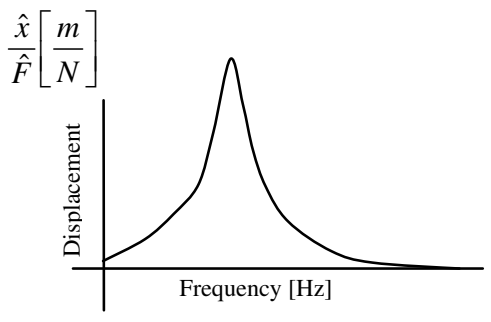

Figure 4.4 System's transfer function by dividing output and input amplitudes per frequency

The system can also be solved analytically. For a linear system the output $u$ will also be harmonic with an amplitude $\hat{u}$ and a phase angle $\varphi$ :

$$
\begin{gathered}
x=\hat{x} \cos (2 \pi f t-\varphi) \\
\dot{x}=-2 \pi f \hat{x} \sin (2 \pi f t-\varphi) \\
\ddot{x}=-4 \pi^{2} f^{2} \hat{x} \cos (2 \pi f t-\varphi)
\end{gathered}
$$

The equation of motion (4.1) thus reduces to an equation with only two unknowns: the amplitude $\hat{x}$ and the phase angle $\varphi$ :

$$
\begin{gathered}
-m 4 \pi^{2} f^{2} \hat{x} \cos (2 \pi f t-\varphi)-c 2 \pi f \hat{x} \sin (2 \pi f t-\varphi)+k \hat{x} \cos (2 \pi f t-\varphi)= \\
=\hat{F} \cos (2 \pi f t)
\end{gathered}
$$

By resolving this equation in an in-phase $(\cos (2 \pi f t))$ and an out-of-phase $(\sin (2 \pi f t))$ part, equation (4.3) can easily be solved. The results are shown in Figure 4.3 and Figure 4.4.

The transfer function provides a direct relationship between input amplitude and output amplitude for each frequency. As the system is fully linear, an external load which is built up from several harmonics can be transformed into response harmonics by which the combined multi-harmonic response can be determined. Figure 4.5 shows the multiple harmonic excitation making up a random excitation with their separate single harmonic responses making up the random response to the random excitation. 

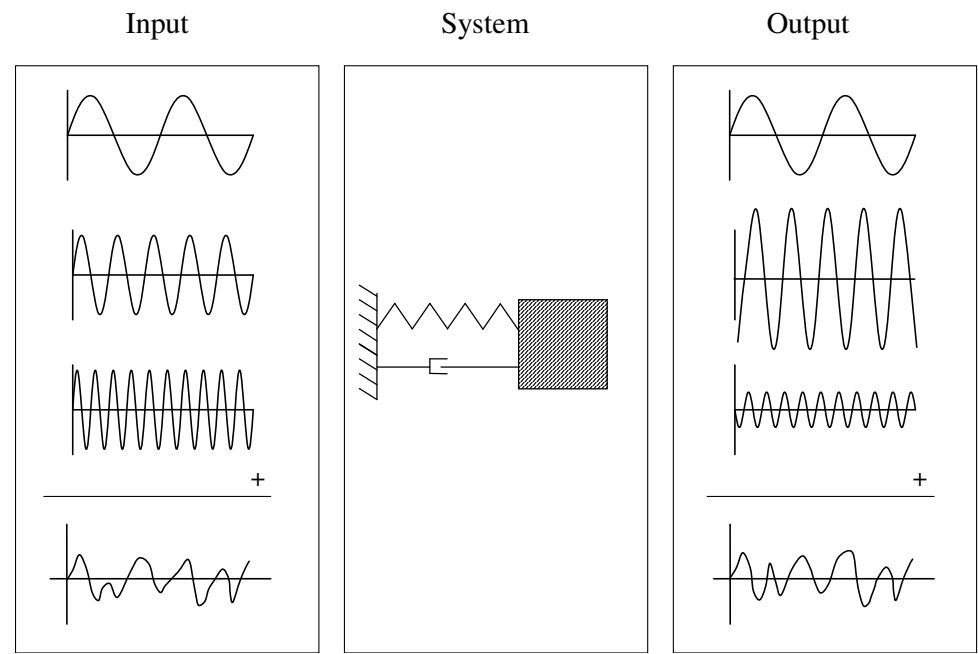

Figure 4.5 Combination of harmonic excitations creating random excitation with accompanying harmonic responses and the combined random response to the random excitation

To transform the time series shown in Figure 4.5 to the frequency domain, both the random input and the random output can be translated into a spectrum through Fast Fourier Transforms (FFT). The relationship between the input and the output spectrum is the transfer function squared. This follows from the definition of spectral density (see section 2.3):

$$
\begin{gathered}
S_{x}(f)=\lim _{\Delta f \rightarrow 0} \frac{1}{2} \frac{[\hat{x}(f)]^{2}}{\Delta f}= \\
\lim _{\Delta f \rightarrow 0}\left[\frac{\hat{x}(f)}{\hat{F}(f)}\right]^{2} \cdot \frac{1}{2} \frac{[\hat{F}(f)]^{2}}{\Delta f}= \\
{\left[\frac{\hat{x}(f)}{\hat{F}(f)}\right]^{2} \cdot S_{F}(f)}
\end{gathered}
$$

hence:

$$
S_{x}(f)=[T R F(f)]^{2} \cdot S_{F}(f)
$$

and:

$$
\operatorname{TRF}(f)=\frac{\hat{x}(f)}{\hat{F}(f)}=\left[\frac{S_{x}(f)}{S_{F}(f)}\right]^{0.5}
$$


Figure 4.6 shows the frequency domain representation of Figure 4.5. The transfer function can either be derived analytically or numerically from the equation of motion or by taking the square root of the output spectrum over the input spectrum.

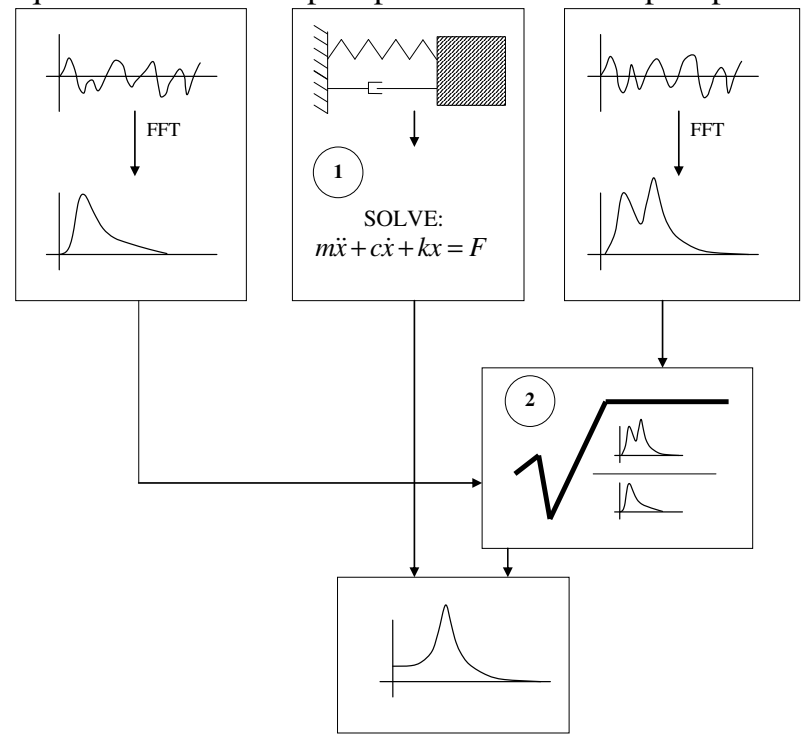

Figure 4.6 Transformation of time series of load and response to spectra; derivation of transfer function through analytical or numerical solution (1) or by the square root of response over excitation spectrum (2)

For simulation in the time domain, the response is calculated by continuously solving the equation of motion at each time step, taking into account the system properties and previous and subsequent time steps through an iteration algorithm. Although a simple 1 DOF system will not require extensive calculations, it can be easily imagined that more complex systems with many degrees of freedom will increase the required computer power.

In the frequency domain, the only relatively intensive computational effort required is the establishment of the transfer function, which is a characteristic property of the system and does not depend on the input. Thus, with the transfer function available, any input spectrum can simply be transformed into an output spectrum.

\subsection{Example application to an offshore wind turbine}

The following example illustrates the application of this modelling technique to real offshore wind turbines. The turbines at Blyth have been subjected to extensive measurement and modelling exercises. Based on the information from the measurements campaigns the structure can be reduced to a model with a mass on top of 
a pole with the mass and inertia, damping and spring stiffness characteristics from the real world.

If desired, the model can be reduced even further to an equivalent 1 DOF system as presented in the previous section. Here, the real system characteristics are used to model the tower top (nacelle) displacement in the direction of the wind. With the methods described earlier, the transfer function between the displacement of the tower top and the loads acting on the tower top can be determined. The reduction of the real turbine to an equivalent 1 DOF system is depicted in Figure 4.7.

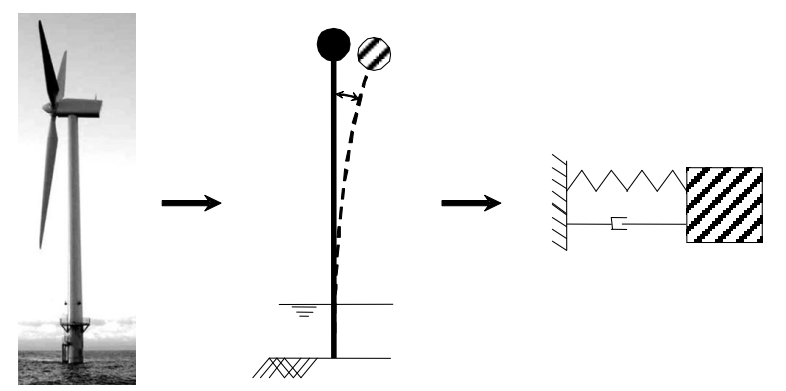

Figure 4.7 Real turbine, modelled as mass-on-pole system and reduced to an equivalent 1 DOF system

The properties required to solve the equation of motion and to create the transfer function are the mass, the spring stiffness and the structural damping. The mass can be found directly from the design data of the actual turbine. Because not all structural mass of the tower will be equally excited, the equivalency of section 2.7 is applied to find the representative mass for modelling the multi-degree-of-freedom system as an equivalent 1 DOF system, equation (4.7), where the tower top mass $m_{\text {top }}=77 \cdot 10^{3} \mathrm{~kg}$ and the tower mass $m_{\text {tower }}=200 \cdot 10^{3} \mathrm{~kg}$ :

$$
\begin{gathered}
m_{\text {rep }}=0.227 m_{\text {tower }}+m_{\text {top }} \\
=0.227 \cdot 200 \cdot 10^{3}+77 \cdot 10^{3} \mathrm{~kg}=122.4 \cdot 10^{3} \mathrm{~kg}
\end{gathered}
$$

The spring stiffness can be determined in several ways. A finite element model can be created. By applying a static top load to the structure and dividing the load magnitude by the top displacement, the spring stiffness $k$ is found. On the real turbine the same method can be applied: connecting a cable between the tower top and an anchor and tensioning the cable with a winch. Figure 4.8 shows a schematic and a photograph of this test. The tests were actually carried out to calibrate the strain gauges; the determination of the spring stiffness was an additional benefit. 

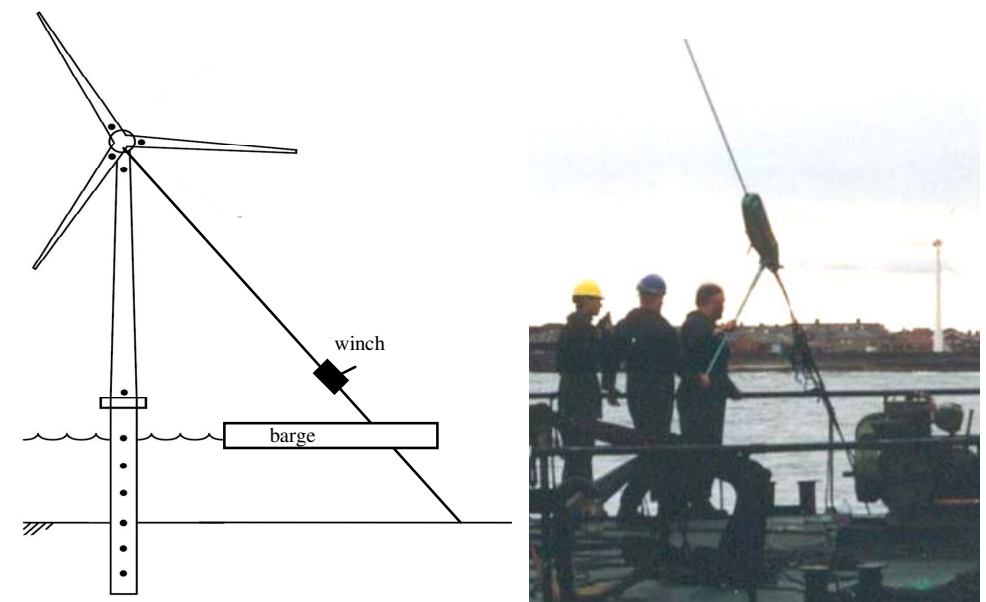

Figure 4.8 Schematic and photograph of applying a top load to the turbine at Blyth to calibrate the strain gauges

A cheaper way of achieving the same is to use the eccentricity of the mass of the nacelle. By switching off the turbine and yawing the nacelle to the desired direction, the strain gauges can also be calibrated. As the anchor used for the previous test appeared to be "crabbing" along the seabed, this latter method was also applied at Blyth.

In this case, the spring stiffness was found with a finite element program, resulting in:

$$
k_{\text {tower }}=\frac{F_{\text {top }}}{x_{\text {top }}}=\frac{1000}{0.00126}=7.94 \cdot 10^{5} \mathrm{~N} / \mathrm{m}
$$

The structural damping cannot be determined as easily. More details on how to assess the damping will be treated in section 6.2. For this example the damping will be taken as $1 \%$ of the critical damping:

$$
\begin{gathered}
\beta=1 \%=0.01=\frac{c_{\text {damping }}}{c_{\text {critical }}}=\frac{c_{\text {damping }}}{2 \sqrt{k_{\text {tower }} \cdot m_{\text {rep }}}} \\
\rightarrow c_{\text {damping }}=(0.01)(2) \sqrt{\left(7.94 \cdot 10^{5}\right)\left(122.4 \cdot 10^{3}\right)}=6235 \mathrm{Ns} / \mathrm{m}
\end{gathered}
$$

The transfer function for tower top load to tower top deflection can now be determined either analytically or numerically using the parameters in equations (4.7), (4.8) and (4.9) in the equation of motion (4.1), resulting in equation (4.10). The resulting transfer function is shown in Figure 4.9. 
$122.4 \cdot 10^{5} \ddot{x}_{t o p}+6235 \dot{x}_{\text {top }}+794 \cdot 10^{3} x_{t o p}=F$

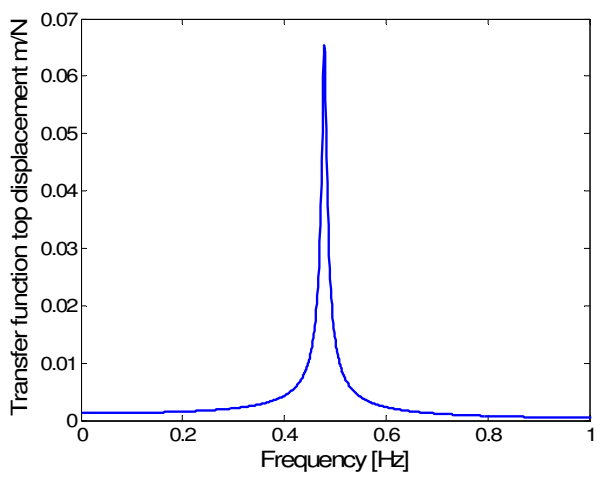

Figure 4.9 Transfer function for tower top load to tower top displacement

To design the support structure for the Blyth turbine, the tower top displacement is most likely not the main design driver, but the bending stress at the mudline. As the mudline bending stress and the tower top displacement can be assumed to be linearly related, the transfer function for tower top displacement only needs to be multiplied by this linear relationship to obtain the transfer function between tower top load and mudline bending stress, as shown in Figure 4.10.

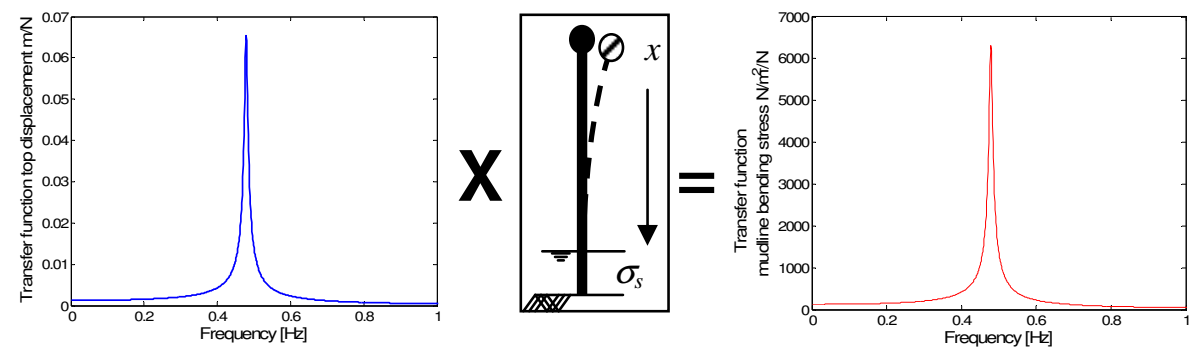

Figure 4.10 Multiplying the transfer function for tower top load to tower top displacement by the linear relationship between tower top displacement and mudline bending stress to obtain the transfer function between tower top load and mudline bending stress

The example shown here uses an equivalent single DOF system of the offshore wind turbine to explain the basic derivation of the transfer function. However, as noted before, in normal design a multi DOF system would be created in a finite element program to find the transfer function for a much more detailed model of the structure. 


\subsection{Linearization of non-linear wave loads}

\subsubsection{Airy wave theory and Morison wave load calculations}

As discussed in section 2.4, the calculation of wave loads on structures consists of two steps:

1. determining the water particle velocity and acceleration

2. calculate the load per section of the structure.

The particle motions can be described by several theories, though in most sea states, the waves can be accurately approximated with a linear theory: Airy. This means that all wave particle velocities and accelerations are linearly dependent on the wave amplitude.

The non-linearity in the wave loads is only introduced by the Morison equation, or more precisely by the drag term in the Morison equation. Equation (4.11) shows the Morison equation (also equation (2.12)) with the square of the velocity component in the drag term:

$$
\begin{gathered}
f(x, z, t)=f_{d}(x, z, t)+f_{i}(x, z, t) \\
f_{d}(x, z, t)=C_{d} \cdot \frac{1}{2} \rho_{\text {water }} D \cdot|u(x, z, t)| u(x, z, t) \\
f_{i}(x, z, t)=C_{m} \cdot \frac{\rho_{\text {water }} \pi D^{2}}{4} \cdot \dot{u}(x, z, t)
\end{gathered}
$$

In the following sections the effect of this non-linear term on the total wave loading on the structure is demonstrated for a monopile support structure.

\subsubsection{Analytical solution for wave loading}

Analytical solutions for the total hydrodynamic load $(F)$ and the overturning moment $(M)$ on a single column of uniform diameter can be obtained by integrating $f_{i}$ and $f_{d}$ from the seabed $z=-d$ to the instantaneous water surface elevation $\zeta$ :

$$
\begin{gathered}
F(x, t)=\int_{-d}^{\zeta}\left\{f_{i}(x, z, t)+f_{d}(x, z, t)\right\} d z \\
M(x, t)=\int_{-d}^{\zeta}\left\{f_{i}(x, z, t)+f_{d}(x, z, t)\right\} \cdot(d+z) \cdot d z
\end{gathered}
$$

The resulting equations [62] can be simplified when the integration extends from the sea floor to the still water level $z=0$. This simplification does not affect the inertia load, which reaches a maximum when the wave surface has a zero crossing, but it discards the additional wave drag load during the passage of the wave crest. Though this effect can be significant if drag load dominates, it will be shown that for the particular application considered here, the simplification is valid. 
The magnitudes of the inertia and drag loads and moments $\left(\hat{F}_{i}, \hat{F}_{d}, \hat{M}_{i}, \hat{M}_{d}\right)$ are then given by:

$$
\begin{gathered}
\hat{F}_{i}=\rho_{\text {water }} g \frac{C_{m} \pi D^{2}}{4} \hat{\zeta} \cdot \tanh k_{\text {wave }} d \\
\hat{F}_{d}=\rho_{\text {water }} g \frac{C_{d} D}{2} \hat{\zeta}^{2} \cdot\left[\frac{1}{2}+\frac{k_{\text {wave }} d}{\sinh 2 k_{\text {wave }} d}\right] \\
\hat{M}_{i}=\rho_{\text {water }} g \frac{C_{m} \pi D^{2}}{4} \hat{\zeta} \cdot d\left[\tanh k_{\text {wave }} d+\frac{1}{k_{\text {wave }} d}\left(\frac{1}{\cosh k_{\text {wave }} d}-1\right)\right] \\
\hat{M}_{d}=\rho_{\text {water }} g \frac{C_{d} D}{2} \hat{\zeta}^{2} \cdot\left[\frac{d}{2}+\frac{2\left(k_{\text {wave }} d\right)^{2}+1-\cosh 2 k_{\text {wave }} d}{4 k_{\text {wave }} \sinh 2 k_{\text {wave }} d}\right]
\end{gathered}
$$

with:

\begin{tabular}{l|l|l}
\hline$\rho_{\text {water }}$ & water density & {$\left[\mathrm{kg} / \mathrm{m}^{3]}\right.$} \\
$g$ & gravitational acceleration & {$\left[\mathrm{m} / \mathrm{s}^{2}\right]$} \\
$k_{\text {wave }}$ & wave number & {$[1 / \mathrm{m}]$} \\
$C_{m}$ & hydrodynamic inertia coefficient & {$[-]$} \\
$C_{d}$ & hydrodynamic drag coefficient & {$[-]$} \\
$d$ & water depth & {$[\mathrm{m}]$} \\
$D$ & column diameter & {$[\mathrm{m}]$} \\
$\hat{\zeta}$ & wave amplitude & {$[\mathrm{m}]$}
\end{tabular}

\subsubsection{Wave load on a single cylinder}

An overview of the critical factors for preliminary design will aid the designer in making a good first estimate of the dimensions of the structure. The monopiles, as they have been and will be constructed in present day projects, are having substantial diameters and will be placed in relatively shallow water coastal regions, which makes a reduction of the full scope of the wave load calculation problem possible.

Examples of North Sea support structures with maximum wave height, associated period and water depth are shown in table 4.1.

Table 4.1 Maximum wave height, water depth and structure dimensions

\begin{tabular}{l||l|l|l|l|l|c} 
& $\begin{array}{c}H_{\max } \\
{[\mathrm{m}]}\end{array}$ & $\begin{array}{c}T_{\text {ass }} \\
{[\mathrm{s}]}\end{array}$ & $\begin{array}{c}\lambda_{\text {wave }} \\
{[\mathrm{m}]}\end{array}$ & $\begin{array}{c}d \\
{[\mathrm{~m}]}\end{array}$ & $\begin{array}{c}D \\
{[\mathrm{~m}]}\end{array}$ & $\begin{array}{c}H_{\max } / D \\
{[-]}\end{array}$ \\
\hline \hline Blyth & 8 & 7 & 50.7 & 8.5 & 3.5 & 2.3 \\
Horns Rev & 8.1 & 12 & 129.4 & 13.5 & 4 & 2.0 \\
OWEZ & 12.8 & 9.5 & 113.3 & 20 & 5 & 2.6
\end{tabular}


Compared to deep water offshore waves, the waves in these coastal areas are relatively short and small by comparison. This fact combined with a relatively large diameter will often result in the dominance of the inertia loads.

The ratios of $\hat{F}_{d}$ to $\hat{F}_{i}$ and $\hat{M}_{d}$ to $\hat{M}_{i}$ is, introducing $H=2 \hat{\zeta}$ are:

$$
\begin{gathered}
\frac{\hat{F}_{d}}{\hat{F}_{i}}=\frac{1}{\pi} \cdot \frac{C_{d}}{C_{m}} \cdot \frac{H}{D}\left[A_{F}+B_{F}\right] \quad \frac{\hat{M}_{d}}{\hat{M}_{i}}=\frac{1}{\pi} \cdot \frac{C_{d}}{C_{m}} \cdot \frac{H}{D}\left[\frac{A_{M}}{B_{M}}\right] \\
A_{F}=\frac{1}{2 \tanh k_{\text {wave }} d} \quad B_{F}=\frac{k_{\text {wave }} d}{2\left(\sinh k_{\text {wave }} d\right)^{2}} \\
A_{M}=\frac{1}{2}+\frac{2\left(k_{\text {wave }} d\right)^{2}+1-\cosh \left(2 k_{\text {wave }} d\right)}{4\left(k_{\text {wave }} d\right) \sinh \left(2 k_{\text {wave }} d\right)} \\
B_{M}=\tanh \left(k_{\text {wave }} d\right)+\frac{1}{k_{\text {wave }} d}\left\{\frac{1}{\cosh \left(k_{\text {wave }} d\right)}-1\right\}
\end{gathered}
$$

Including drag and inertia coefficients $C_{d}=1.0$ and $C_{m}=2.0$ the ratios $\hat{F}_{d} / \hat{F}_{i}$ and $\hat{M}_{d} / \hat{M}_{i}$ become:

$$
0.082 \frac{H}{D}<\frac{\hat{F}_{d}}{\hat{F}_{i}}<0.51 \frac{H}{D} \text { and } 0.096 \frac{H}{D}<\frac{\hat{M}_{d}}{\hat{M}_{i}}<0.51 \frac{H}{D}
$$

The total wave load on the tower is the vector sum of drag and inertia components. Thus, the ratios of total load and moment to the inertia component are:

$$
\frac{\hat{F}_{t o t}}{\hat{F}_{i}}=\sqrt{1+\left(\hat{F}_{d} / \hat{F}_{i}\right)^{2}} \text { and } \frac{\hat{M}_{t o t}}{\hat{M}_{i}}=\sqrt{1+\left(\hat{M}_{d} / \hat{M}_{i}\right)^{2}}
$$

This results in the following ranges of extreme total wave load and moment relative to the wave inertia load and corresponding moment shown in table 4.2. 
Table 4.2 Ratio of total load and moment to inertia load and moment for different $H / D$ ratios

\begin{tabular}{c|c|c}
$H / D$ & $\hat{F}_{t o t} / \hat{F}_{i}$ & $\hat{M}_{t o t} / \hat{M}_{i}$ \\
\hline \hline 1 & $1.00<\hat{F}_{t o t} / \hat{F}_{i}<1.12$ & $1.00<\hat{M}_{t o t} / \hat{M}_{i}<1.12$ \\
2 & $1.01<\hat{F}_{t o t} / \hat{F}_{i}<1.43$ & $1.02<\hat{M}_{t o t} / \hat{M}_{i}<1.43$ \\
3 & $1.03<\hat{F}_{t o t} / \hat{F}_{i}<1.83$ & $1.04<\hat{M}_{t o t} / \hat{M}_{i}<1.83$
\end{tabular}

This demonstrates that with the diameters as applied for monopile support structures of wind turbines the extreme wave loading is mainly due to inertia. In several cases the drag load may be ignored altogether for initial design purposes, while this being considered too rough an approximation, the total load and moment can still be related to the inertia load by applying a multiplication factor. For less extreme wave conditions the dominance of the inertia loading is only more pronounced. This means that any wave induced fatigue will be governed by linear inertial wave loading.

\subsubsection{Graphic representation of wave loads}

Following this analytical derivation a more ready-to-use version of the wave loads can be compiled. Graphs representing the inertia and the drag loads and moments, respectively, based on the wave period for different water depths from 5 to $30 \mathrm{~m}$ in $5 \mathrm{~m}$ intervals have been determined using equations (4.13).

Both load and moment amplitudes are plotted in a normalised form by eliminating the influence of the cylinder diameter and the wave amplitude, thus explicitly showing the influence of wave period (wave length) and water depth. The normalised loads and moments are of the following form:

$$
\begin{aligned}
& \frac{\hat{F}_{i}}{D^{2} H} \quad \text { and } \quad \frac{\hat{F}_{d}}{D H^{2}} \quad\left(\mathrm{kN} / \mathrm{m}^{3}\right) \\
& \frac{\hat{M}_{i}}{\hat{F}_{i} \cdot d} \text { and } \frac{\hat{M}_{d}}{\hat{F}_{d} \cdot d} \quad \text { (non-dimensional) }
\end{aligned}
$$

The plots in 0 have been made using hydrodynamic coefficients with values of $C_{m}=$ 2.0 and $C_{d}=1.0$, while the density of seawater is set at $\rho_{\text {water }}=1025 \mathrm{~kg} / \mathrm{m}^{3}$. Due to the simple proportionality of loads and moments with the hydrodynamic coefficients, the loads for other values than 2.0 and 1.0 are obtained by simple multiplication. Full page graphs are plotted in appendix I. 

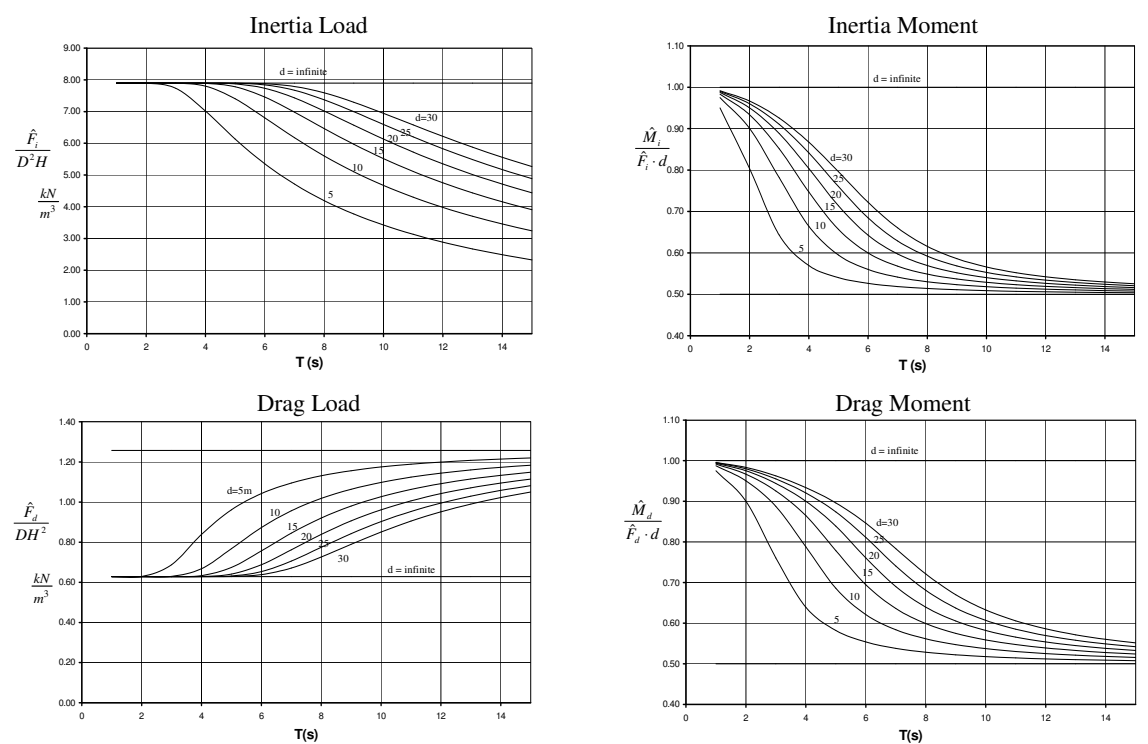

Figure 4.11 Wave induced inertia and drag loads and moments as a function of wave period

\subsection{Frequency domain determination of fatigue damage due to waves}

In the previous sections of this chapter the analysis of the dynamic response of a structure and the calculation of wave loads have been dealt with. By combining these, the frequency domain method for wave induced fatigue can be completed. The wave spectrum describes the input wave field and by multiplying that by the square of the transfer function for the bending stress, the response spectrum of bending stress is determined. Section 4.4 showed that for large diameter monopiles in not too deep water, the non-linear drag load does not have much influence on the total load. Usually the drag load is calculated for a specific wave height per frequency and included as a linear term in the transfer function. Offshore design software offers several options for these linearizations, but these will not be discussed here.

Figure 4.12 shows the calculation of the transfer function and the method of determining the bending stress spectrum for a given wave spectrum. This method can be applied to all wave spectra summarized in a scatter diagram, making the calculation of the fatigue damage very fast. 

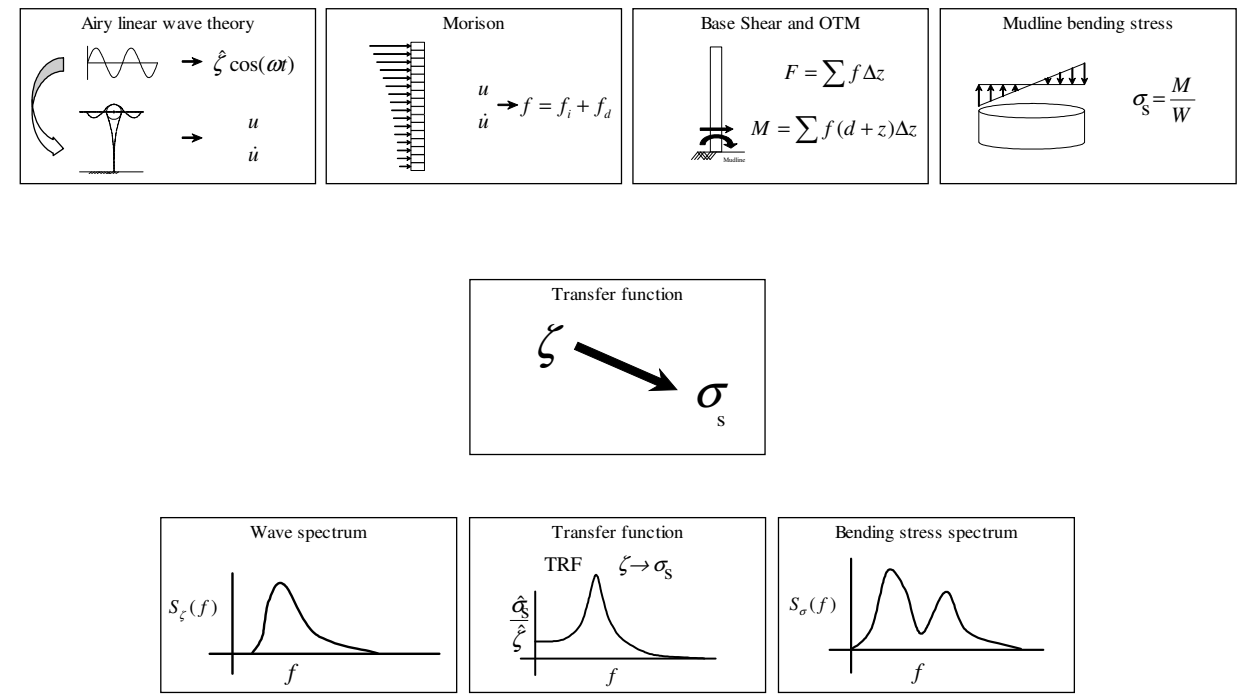

Figure 4.12 The sequence of calculating wave particle kinematics with Airy linear wave theory to wave loads with Morison, summed to overturning moment and transformed to bending stress

\subsection{Conclusions}

Frequency domain and time domain calculation methods of linear systems are two different analyzing techniques of the same system, which are entirely interchangeable. The major difference is that every time domain representation is a specific realisation of a stochastic process, while the frequency domain representation covers all stochastically possible realisations.

When calculating wave loads on a structure, the Morison equation introduces a nonlinearity through the drag term. This would tend to make the calculation of wave loads in the frequency domain less straightforward. However, for monopile structures of the dimensions typically used for offshore wind turbines, it has been shown that the linear inertia term is dominant over the non-linear drag term. This is true for the maximum waves at the reference sites and even more so for the much smaller waves that induce most of the fatigue loading. This means that the system is indeed nearly linear and that by using a linearized frequency domain approach, the fatigue damage cannot only be assessed very quickly, but also without loss of accuracy compared to time domain calculations. 


\section{Devising a frequency domain method for offshore wind turbine fatigue}

\subsection{Introduction}

Presently, design calculations for offshore wind turbines are carried out in the time domain. All non-linear load-structure interactions and control features can be incorporated to a sufficient level of detail to enable a comprehensive analysis of a design and which is assumed to provide reliable results. To obtain correct answers, however, these time domain calculations need complete and complex models of aerodynamics, control, generator, etc. and require a large number of relatively time consuming calculations for a fatigue damage check. For the offshore contractor, responsible for the design of the support structure, detailed models of the turbines are not always available, even if the contractor would be the owner of specialist turbine simulation software and would have qualified personnel to use it correctly.

As shown in the previous chapter, frequency domain calculation of fatigue of offshore structures is common practice. If the turbine loads could be incorporated in the frequency domain methods, the design practice would be aided tremendously, in both reducing the required effort and increased understanding of the design. Furthermore, a frequency domain fatigue calculation method will enable rapid design of location specific structures and quick parameter studies.

This chapter describes the basic outline of a frequency domain method for offshore wind turbine fatigue determination. In section 5.2 a general comparison between time domain fatigue due to wind loads only and the frequency domain method is shown. The frequency domain approach is further detailed in section 5.3. As the turbines under consideration are to be designed for offshore, section 5.4 describes the method to combine the outcome of frequency domain wind response calculations and wave induced response calculations. In section 5.5 the methods to derive fatigue from the combined stress response spectra are treated. For a comparison of the fatigue damage calculated using time and frequency domain, consistent use of time domain simulations is required. Section 5.6 gives an overview of expert opinions on these setting. Section 5.7 gives the conclusions of this chapter.

\subsection{Similarities between time and frequency domain approaches}

The steps required to carry out a fatigue calculation are depicted in Figure 5.1. In the time domain, on the left hand side, the wind characteristics are used as input to create a three-dimensional turbulent wind field. This field is then "shoved" through the rotor 
disk in a time domain model of the turbine. The program incorporates all specific details: wind shear, tip corrections, wake influence, tower shadow, etc. The program produces time series of bending moments and other relevant responses for different stations along the support structure.

To calculate fatigue damage at a specific location, the stress time series is postprocessed. First, the bending moment is converted to bending stress, then the stress is rainflow counted (RFC) to find the stress range variation histogram. With the proper $S-N$ curve for the detail under consideration, the Miner sum gives the fatigue damage value $D_{\text {fat }}$.

The frequency domain fatigue calculation method is shown on the right in Figure 5.1. The different steps roughly follow the procedures of Turbu and Turbload as described in section 3.7 and serve as a first outline of the new frequency domain method which will be detailed in the next sections.

The purpose of use of the frequency domain method is to arrive at an efficient tool to be able to optimise the design of the support structure. This requires complete separation between turbine and support structure in the calculation method. Figure 5.1 shows the separate calculation of the turbine loads in step 1 and the derivation of the transfer function between tower top load and support structure bending stress in step 3 .

Previous studies [3] [4] [34] [48] [49] have demonstrated the effect the operating RNA has on the support structure dynamics. The rotor introduces aerodynamic damping, which should be taken into account in the further analysis of the structure, step 2.

Steps 1 to 3 result in a transfer function per wind class for a turbulent wind field to support structure bending stress at any desired location along the structure. By multiplying the square of this transfer function with the input turbulent wind spectrum, the stress response spectrum can be found. Using this spectrum, several counting methods can be used to derive the fatigue damage as shown in step 4 . 


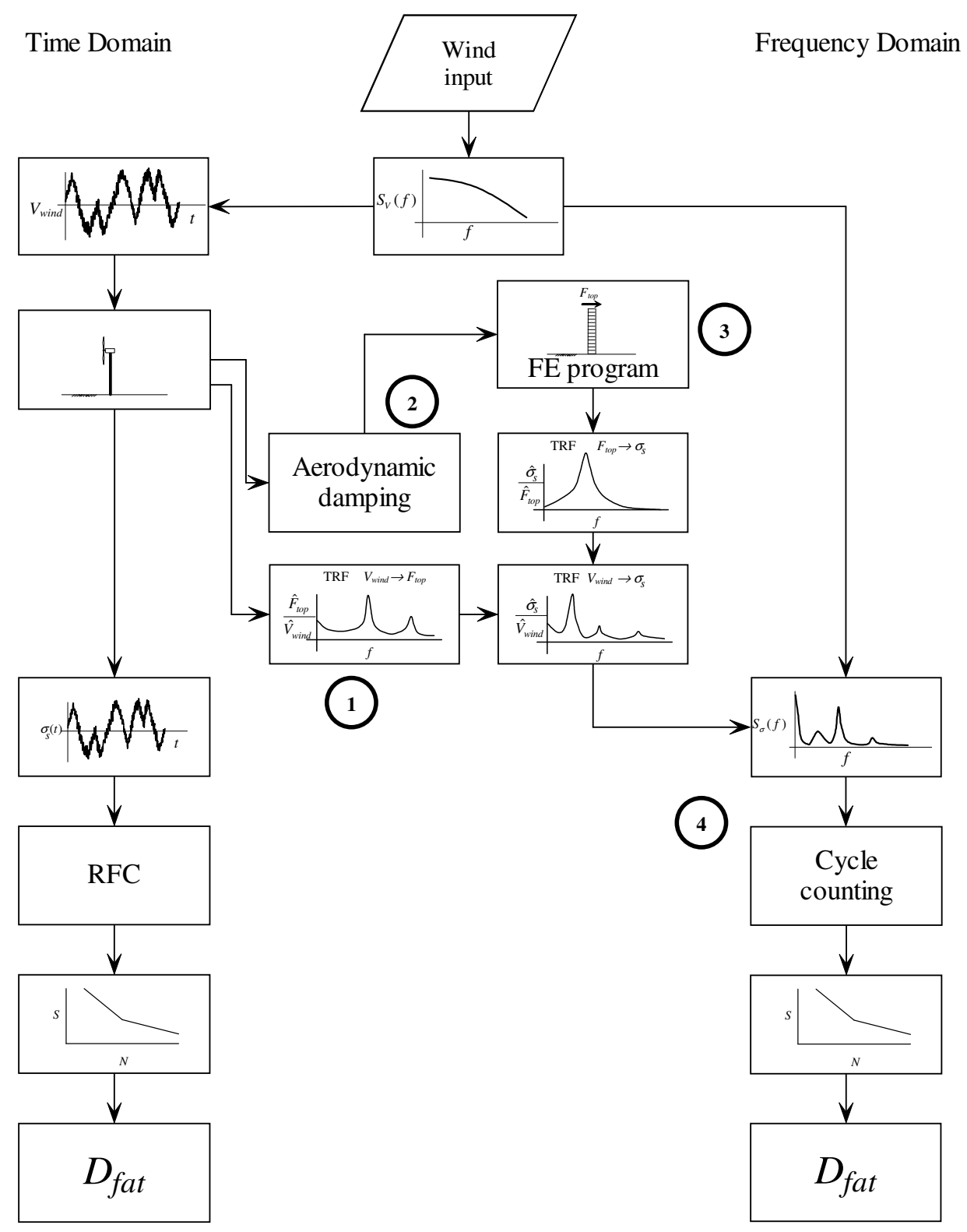

Figure 5.1 Fatigue calculation method in time domain (left) and frequency domain (right) 


\subsection{Determining stress response spectra under wind loading}

\subsubsection{Transfer function between wind field and tower top load}

The non-linear behaviour of the wind turbine in different wind conditions cannot easily be linearized in an integral and direct manner. Some form of time domain simulation to solve the blade element momentum equations for different wind conditions will usually be required. The same approach is used here in a pragmatic way. During or preferably before the design of an offshore wind farm, a turbine manufacturer is selected. It can be assumed that the manufacturer has a working computer model of his turbine, capable of performing all typical design calculations prescribed for normal turbine design. To uncouple the turbine calculations from the behaviour of the support structure, the structure can be modelled as a rigid structure.

By modelling the support structure completely rigid, no support structure dynamics will occur. This makes the transfer functions for wind speed to tower top load universally applicable to any kind of support structure. The rigid modelling completely uncouples the turbine and the support structure.

The program can now be used to calculate time series of the tower top load due to specific wind conditions described by wind turbulence spectra with a mean wind speed, turbulence intensity and wind shear. As output, the tower top load is recorded. When the time series of both the input wind field and the tower top load are converted to a spectrum, the transfer function can be derived by dividing both spectra and taking the square root:

$$
T R F=\sqrt{\frac{S_{F_{t o p}}(f)}{S_{V}(f)}}
$$

For $S_{V}(f)$ e.g. the Von Kármán or the Kaimal spectra can be used; see section 2.5.

\subsubsection{Aerodynamic damping}

For an operating RNA the support structure motion and turbine aerodynamics have a significant effect on each other. When the turbine moves forward (against the wind), the blades experience an increase in total wind speed. As a result of this increased wind speed, the instantaneous tower top load is increased through basic aerodynamic action of the blades. This load is acting against the tower top motion. For backward motion, the situation is analogous, now resulting in a reduced tower top load, also reducing the tower top motion. This effect is known as aerodynamic damping.

To enable separation of RNA and support structure calculations in the frequency domain approach, the aerodynamic damping needs to be calculated for all wind speed 
classes and must be incorporated in the dynamic model of the support structure through an additional viscous damping.

Aerodynamic damping has only been described in a few publications. Its explicit calculation was never required with the use of time domain simulation programs, which incorporate the effect intrinsically. A closed-form linearization made by Garrad was given in [49]. Later, Kühn [4] describes Garrad's method and two other methods and compares the results. A further overview and application of the methods for variable speed turbines is given in [80].

For this thesis, the following methods are distinguished, specifically named to prevent confusion:

- Garrad method, a closed-form linearization

- Numerical linearization, based on a state-space analysis

- Non-linear simulation, a full time domain simulation analysis

- Engineering estimate, a single engineering number.

\section{The Garrad method}

In Freris [49], Garrad published the derivation of a closed-form linearization. By solving the blade element part of the blade element momentum equation for a small change in the wind speed, the damping can be calculated. The solution is based on the following assumptions:

- the tip speed is much larger than the wind speed

- the tip speed is much larger than the tower top motion

- the lift coefficient $C_{L}$ is much larger than the drag coefficient $C_{D}$

Using these assumptions, the aerodynamic damping can be found with:

$$
\beta_{\text {aero }}=\frac{N_{b} \rho_{\text {air }} \Omega}{8 \pi f_{\text {nat }} m_{\text {rep }}} \int_{R_{\text {root }}}^{R_{\text {itp }}} \frac{d C_{L}}{d \alpha} c_{a}(r) r d r
$$

with:

\begin{tabular}{l|l|l}
\hline$N_{b}$ & Number of blades & {$[-]$} \\
$\rho_{\text {air }}$ & density of air & {$\left[\mathrm{kg} / \mathrm{m}^{3}\right]$} \\
$\Omega$ & rotor angular velocity & {$[\mathrm{rad} / \mathrm{s}]$} \\
$f_{\text {nat }}$ & natural frequency of the support structure & {$[\mathrm{Hz}]$} \\
$m_{\text {rep }}$ & representative mass for a 1 DOF system & {$[\mathrm{kg}]$} \\
$R_{\text {tip }}$ & radius of the blade tip & {$[\mathrm{m}]$} \\
$R_{\text {root }}$ & radius of the blade root & {$[\mathrm{m}]$} \\
$C_{L}$ & lift coefficient & {$[-]$} \\
$\alpha$ & angle of attack & {$[\mathrm{deg}]$} \\
$c_{a}(r)$ & blade chord at position $r$ & {$[\mathrm{~m}]$} \\
$r$ & radial position of blade element & {$[\mathrm{m}]$}
\end{tabular}


Solving equation 5.2 still requires the solution of the blade element momentum theory for each wind speed to find the derivative of the lift coefficient over the angle of attack including the steady state induction factor for that angle of attack. In the application here, the ratio inclination of the lift curve was taken as a constant. The advantage of the closed-form solution is that the calculation can be performed without the need for a complete wind turbine simulation program.

In any mathematical program, e.g. Matlab or even Excel, an iterative blade element momentum theory can be programmed to find the aerodynamic damping. Cerda Salzmann developed a Matlab tool, RECAL, to calculate offshore wind turbine responses to wind and wave loads [55]. To validate the tool, the offshore wind turbine at Blyth was modelled. The program gave good results with respect to the real turbine performance. The program not only calculates the power curve correctly, also the wind speed dependent pitch angle and the variable rotor speed were found to match measurements nicely.

A large advantage of the tool is the minimum amount of input data required. For modelling of the blade aerodynamics, the number of airfoils has been reduced from seven to only one, with no noticeable loss in accuracy. With the validated steady state pitch angles and rotational speed for each wind speed, the aerodynamic damping is found.

\section{Numerical linearization}

To fine-tune the controls of a wind turbine, control engineers use linearization methods to derive the state-space description of the entire turbine or parts of it for specific conditions. The computer program DUWECS, which Kühn [4] used, was equipped with such a linearization option. For the turbine simulation program used in this thesis (Bladed) such an option can be purchased separately, but was not available during this work. The method was used by Garrad Hassan and partners in the OWTES project [3] to find the aerodynamic damping for simulations of the Blyth turbines. The method is not used here.

\section{Non-linear time domain simulation}

This method is straightforward: run a normal time domain simulation with all features of turbulence, etc. and add a constant horizontal load on the nacelle which is released after transient effects of the simulation have died away. By running the same simulation again with exactly the same wind time trace but without the top load, the top loads of both simulations can be subtracted leaving the response due to the release of the single load. This results in a decaying vibration from which the logarithmic damping decrement can be estimated. This method could be executed in DUWECS, which was available to Kühn [4]. 
Bladed does not offer the opportunity to add a top load. Therefore a different approach was used in [80]. Per time step the incident in-plane wind speed per blade section $V_{\text {section }}$ is recorded together with the resulting response thrust force $F_{a x \text {,section }}$. The damping was calculated by dividing the increase of the thrust per increase of in-plane wind speed:

$$
c_{\text {damping }}=\frac{\Delta F_{\text {ax,section }}}{\Delta V_{\text {section }}}
$$

It was found that for each blade section, the aerodynamic damping ratio of the scatter of thrust change over wind speed change is approximately constant as shown in Figure 5.2 .

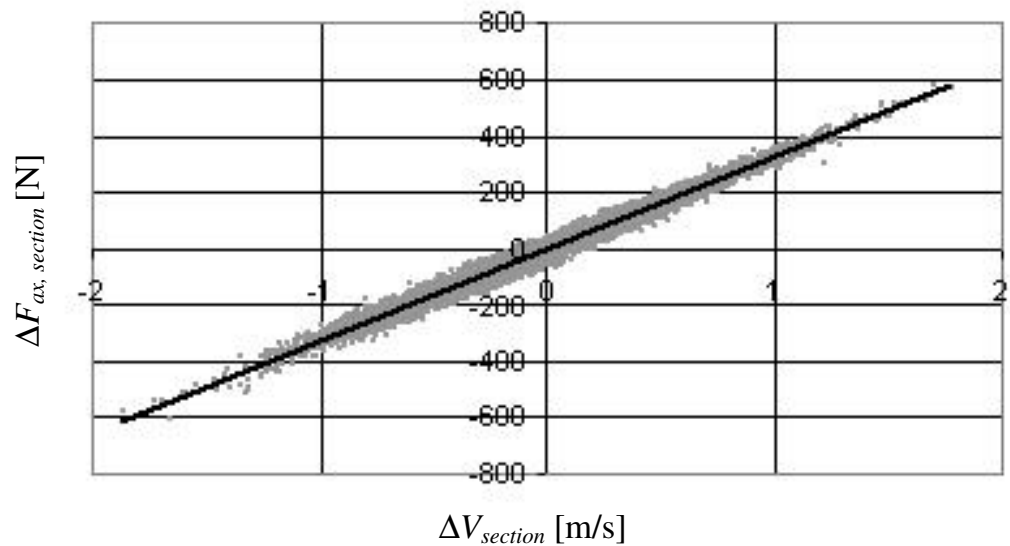

Figure 5.2 Aerodynamic damping estimation for thrust change over wind speed change for one blade section in a non-linear time domain simulation [80]

As the integration of the aerodynamic damping per section for the entire blade was not found feasible in Bladed within the scope of [80], a pragmatic approach was chosen to use the RECAL non-linear simulation tool that uses only a single time varying wind signal for the entire rotor and a single airfoil for the entire blade. The aerodynamic damping was then calculated as the increase of the total rotor thrust over the increase of the incident wind speed.

The full potential of this method has not yet been utilized and more work in this field is required.

\section{Engineering estimate}

It would be ideal when the aerodynamic damping per wind speed could become a generally accepted turbine characteristic, published in the turbine technical 
specifications sheet, like the power curve and component weights. This would prevent offshore contractors having to do complex modelling with any of the methods presented here. In the mean time, to facilitate preliminary design engineering, a crude estimate of the aerodynamic damping for all wind speed classes could be used.

For this thesis the engineering estimate is set at $4 \%$ aerodynamic damping. This figure is based on the outcome of the previous methods and a good deal of gut-feel. The variation with wind speed is neglected.

\subsubsection{Dynamic behaviour of the support structure}

In step 3, the support structure is processed in a finite element model (FEM). To include for the effect of aerodynamic damping, the structural damping factor is increased with the value derived in step 2. Solving the equations of motion for one or more specific points along the structure under time varying tower top loads results in a transfer function between tower top load and bending stress at those specific locations.

The total transfer function between the turbulent wind field and the bending stress can now be found as the product of the derived transfer functions from steps 1 and 3. By multiplying the input wind spectrum with the combined transfer function squared, we find the total bending stress spectrum for the location under consideration. To validate the method, this spectrum can be compared with the spectrum found from a time series of bending stress created by a full non-linear time domain simulation in Bladed under the same conditions.

The details of the entire sequence for steps 1,2, 3 in the frequency domain approach is shown by the flowchart in Figure 5.3. In chapter 6 the different steps are executed and described for the Blyth turbine model. 


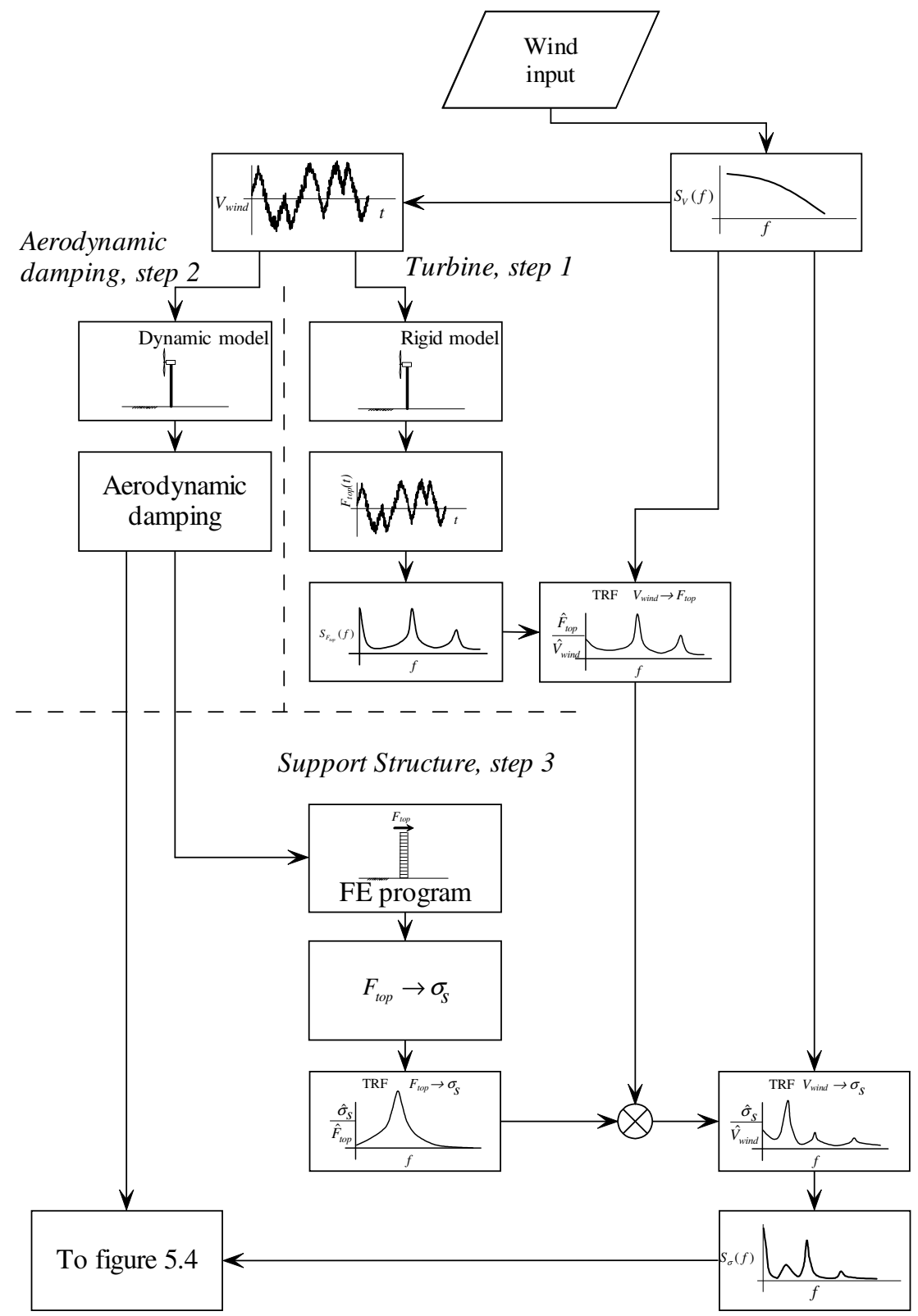

Figure 5.3 Flowchart of the frequency domain calculation to determine the stress spectrum due to wind loading 


\subsection{Incorporating stress response due to wave excitation}

The previous section presented a method to derive the response spectrum for bending stress in the support structure due to wind loads on the rotor. In chapter 4 , the frequency domain method for response calculations due to wave excitation used for the design of offshore oil \& gas structures was presented. If the responses due to wind and wave excitation are assumed to be fully independent, the combined response can be determined by adding the respective response spectra. The effectiveness of this method was already shown in [34]. The only interaction between the wind turbine and the response of the support structure due to wave excitation is the aerodynamic damping. This damping can easily be included in the wave response calculation using the magnitude of the aerodynamic damping as calculated in section 5.3.2. The flowchart in Figure 5.4 shows the steps for combining the stress response spectra due to wind and wave loading to obtain the spectrum of the total stress response.

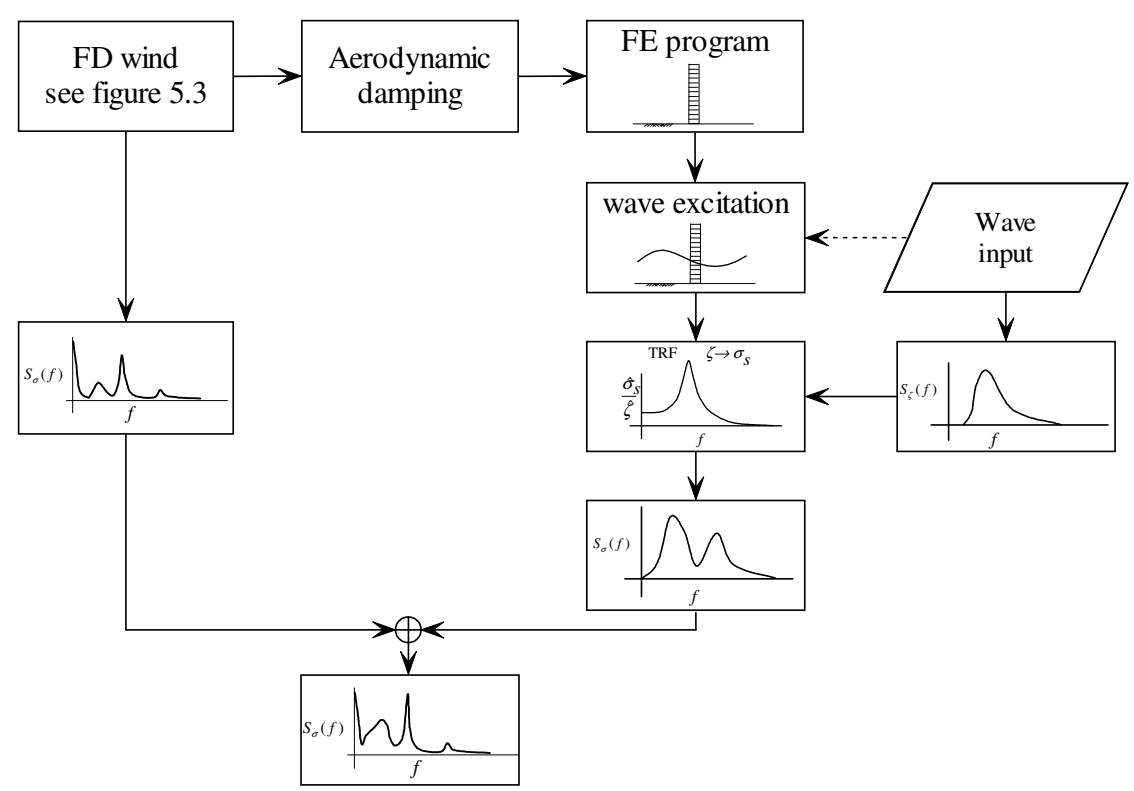

Figure 5.4 Flowchart showing the combination of stress spectra due to wind and wave loading

\subsection{Fatigue damage calculation using the spectra of the total stress response}

For all locations of interest, the method presented in the previous sections will provide the response spectra for the total stress due to combined wind and wave loading. The final step is to process this spectrum to obtain the cumulative fatigue damage $D_{f a t}$. As 
determined in section 2.8, several solutions exist to derive the stress range distribution from the spectral moments of a stress spectrum. Three methods will be applied in this thesis: Rayleigh, Rice and Dirlik. Alternatively, a spectrum can always be transformed back to the time domain allowing rainflow counting. This may look contra-productive: inventing a frequency domain solution and then turning back to the time domain, but valid reasons may exist however:

- Rainflow counting is the industry standard in wind energy, and is incorporated in this thesis for reference.

- $\quad$ TURBU and Turbload, as well as the frequency domain offshore program SESAM [67] incorporate tools to perform IFFT and rainflow counting on response spectra.

The different steps to determine fatigue damage are shown in Figure 5.4. The execution of these steps has been described in chapter 6 .

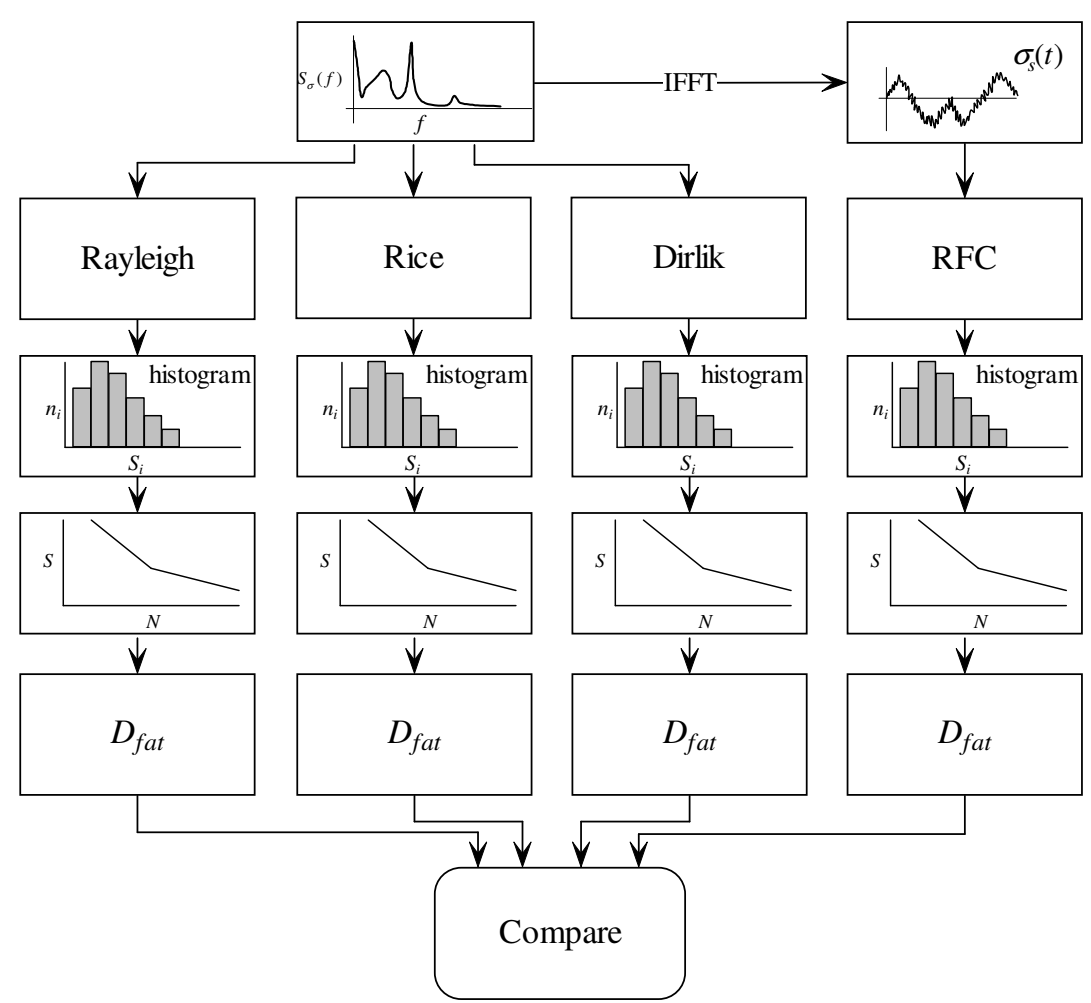

Figure 5.5 Fatigue damage calculation in the frequency domain using three probability distributions for stress peaks (ranges) and in the time domain using rainflow counting 


\subsection{Expert opinions on fatigue damage check in the time domain}

For a fatigue damage check all environmental states need to be considered and the associated fatigue damage must be calculated and subsequently summed. Sofar the industry has not reached consensus on the details of fatigue analysis of support structures for offshore wind turbines is not an area of consensus. Design standards only give directions that can be interpreted in various ways. Fortunately, brief interviews with representatives of four leading offshore wind turbine design consultants/certification agencies revealed more or less consensus in the way to proceed [78][81] [82] [83] .

The goal of the interviews was to agree on a consistent set of simulation parameters for the time domain simulations, the result of which will be used to compare with the frequency domain method results.

\section{Time step}

There is a clear and consistent opinion on this matter: all four respondents prescribed a time step of $0.1 \mathrm{~s}$, although the value seemed to be more experience-based than supported through analysis of the dynamic systems at hand.

In section 6.2.2 the time step is determined based on the calculation requirements following the flowchart of figure 2.7 .

\section{Simulation length}

The standard simulation length for turbine design is 10 minutes, $600 \mathrm{~s}$. All respondents felt that for combined wind and wave load simulation longer simulations should be carried out, varying from 1200 to $3600 \mathrm{~s}$, or more simulations of $600 \mathrm{~s}$ length for the same environmental state. The IEC 61400-3 states 3600s or 6 times 600s [65]. Another option proposed was not to reduce the number of environmental states and then assume that the number of states includes favourable and unfavourable realisations in the large set of states under consideration, as long as the random seed on which the IFFT is based was different for each load case. This would in itself promote statistical stability of the results.

\subsection{Conclusions}

In this chapter a frequency domain analysis method has been presented for fatigue damage calculations of the support structure of offshore wind turbines due to wind and wave loading. The turbine is first modelled on a rigid support structure and for every wind speed class the tower top loading is simulated in the time domain. From the spectra of the input time varying wind speed and the output tower top loading due to wind the associated transfer function is determined. 
Next, the support structure is modelled separately in a finite element model. For the turbine generated wind loading, a transfer function is determined for tower top loading to bending stress at the hot spot under consideration for the fatigue assessment. For wave loading a similar transfer function is created. The effect of the operating rotor on the support structure dynamics is accounted for through the aerodynamic damping, which is assessed separately and incorporated as additional modal damping in the support structure model.

The stress variations due to turbine generated wind load can now be calculated through combination of the tower top load and tower stress transfer functions per wind speed class. The stress variations due to wave loading are found through multiplication of the input wave spectrum and the wave stress transfer function. Assuming that wind and wave induced stresses are independent, both stress response spectra are summed to find the total stress response at the hot spot under consideration.

From stress response spectra, the fatigue damage can be determined through several methods. These methods will be compared in the next chapter in which the proposed frequency domain method will be applied to a model of the offshore wind turbines at Blyth. 


\section{Application of frequency domain fatigue to Blyth}

\subsection{Introduction}

In this chapter, the different steps are described to create response spectra of bending stresses in the support structure of an offshore wind turbine due to wind loads on the turbine and due to the combined wind loads and wave loads on the support structure. In section 6.2 the test model is described and the simulation parameters are determined. The subsequent section shows the steps to calculate the transfer function between turbulent wind input and bending stress. Only the stress at the mudline is analysed to validate the method. Calculation of stresses at other locations of the support structure are completely similar. The transfer function is found through time domain simulation of the wind field in Bladed and frequency domain analysis of the support structure in SESAM (section 6.5). Section 6.4 describes the derivation of aerodynamic damping and the impact of adding this effect to the frequency domain model. In section 6.6 the frequency domain method is applied to all wind speed ranges and the output is compared with time domain results. Furthermore, the results of the frequency domain method are compared with measurements from the real offshore wind turbine at Blyth. Section 6.7 combines the stress spectrum due to wind loading and stress spectrum due to wave loading. The results are compared with time domain simulations as well as with measurements at Blyth. The resulting stress response spectra are post-processed to find the fatigue damage in section 6.8. The outcome is compared with the results from the time domain simulations. The final section 6.9 gives the conclusions on the application of the frequency domain method on the Blyth offshore wind turbines.

\subsection{Description of the simulation model and parameter settings}

\subsubsection{Description of the as-built offshore wind turbines at Blyth}

The offshore wind turbines at Blyth have been subjected to an extensive measurement programme as part of the EU sponsored OWTES project. These results have been processed to 580 campaigns, which were available for this thesis. Figure 6.1 shows the layout of the measuring equipment on the southern turbine [3]. 


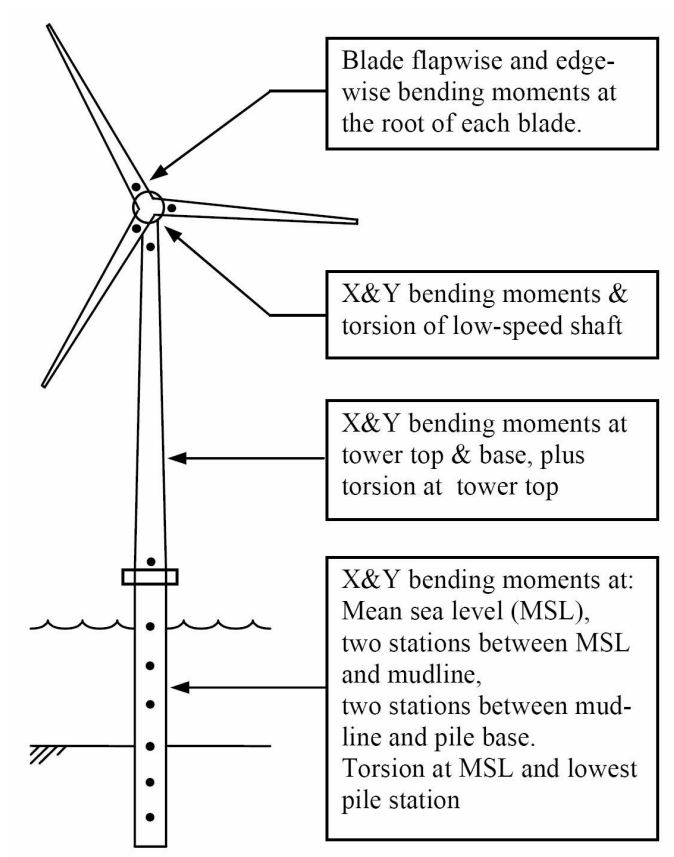

Figure 6.1 Measuring equipment on the southern turbine at Blyth [3]

The turbine has been modelled in Bladed by Garrad Hassan with assistance of the turbine manufacturer Vestas, who provided all aerodynamic, generator and control properties. The turbine model was found to compare very well with the performance of the actual turbine [47]. Figure 6.2 shows the comparison between the model and the measured values for power production and mudline bending moment.
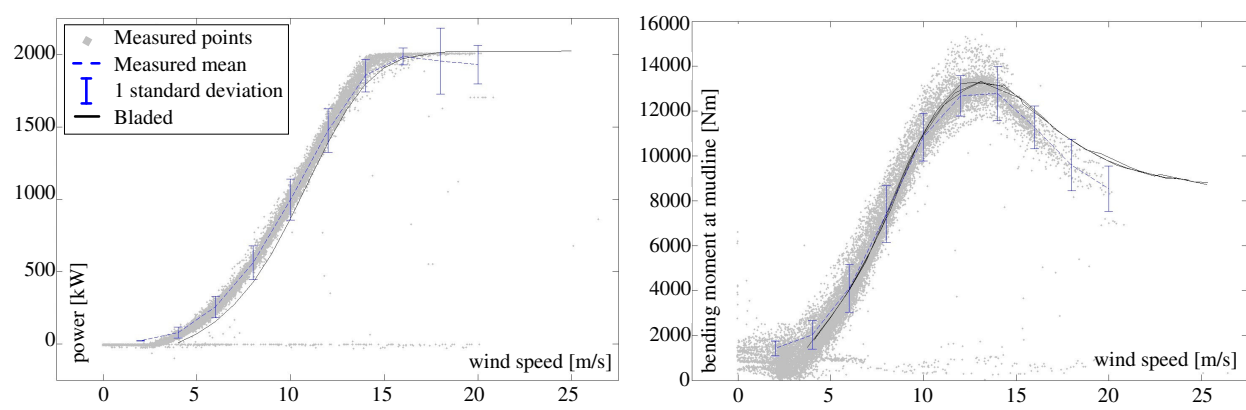

Figure 6.2 Comparison between measurements and simulations of the Blyth turbine for the power production (left) and the mudline bending moment (right) [47] 
A crucial point in stress calculations (and structural analysis in general) is the correct modelling of the structural properties. Based on the available data of the support structure, the first natural frequency could be found accurately (within 2\%). The second natural frequency shows a slightly larger deviation with the measured value (about 3\%), but the effect of this error on the structural response is low, as will be shown later in this chapter. Table 6.1 shows the four lowest natural frequencies of the support structure, for both the real structure and the models [47].

Table 6.1 Measured and modelled natural frequencies of the Blyth turbine

\begin{tabular}{l|c|c} 
& $\begin{array}{c}\text { Model frequency } \\
\text { in } \mathrm{Hz}\end{array}$ & $\begin{array}{c}\text { Measured } \\
\text { frequency in } \mathrm{Hz}\end{array}$ \\
\hline \hline $1^{\text {st }}$ fore-aft & 0.47 & 0.48 \\
$1^{\text {st }}$ side-side & 0.47 & 0.48 \\
$2^{\text {nd }}$ fore-aft & 3.1 & 3.0 \\
$2^{\text {nd }}$ side-side & 2.9 & 2.9
\end{tabular}

A final critical input parameter for structural analysis is the structural damping of the support structure. This property is very difficult to estimate during design. Damping is the process of losing kinetic energy by transferring energy to the air, water and soil and due to internal processes like the production of (minute amounts of) heat and sound. Design standards are not fully aligned on this issue and give guidelines for structural damping between $1 \%$ and $5 \%$ of critical, where the higher damping values can be used for extreme response calculations and the lower damping values are recommended for fatigue damage calculations. Table 6.2 summarizes the preferred values from different design standards.

Table 6.2 Prescribed structural damping as percentage of critical damping by different standards

\begin{tabular}{l|c} 
Standard & $\begin{array}{c}\text { Damping as percentage of } \\
\text { critical damping }\end{array}$ \\
\hline \hline ISO [77] & for fatigue: $1-2 \%$ \\
API [52] & for fatigue: $2 \%$ \\
GL [20] & $1 \%$ \\
DNV [19] & $1 \%$
\end{tabular}

The only way to find the actual damping is to excite the real structure and let the vibration die out in its natural frequency. This can be accomplished for a wind turbine by performing an emergency brake when the turbine is operating. 
The measuring campaign designated $\mathrm{x} 618 \mathrm{~b}$ is a recording of an emergency brake test of the operating turbine [53]. This sudden application of the brake produces an instantaneous excitation of the tower top resulting in a damped vibration in the first mode of the structure as shown in Figure 6.3. The measurement can be used to establish both the first natural frequency and the damping factor.

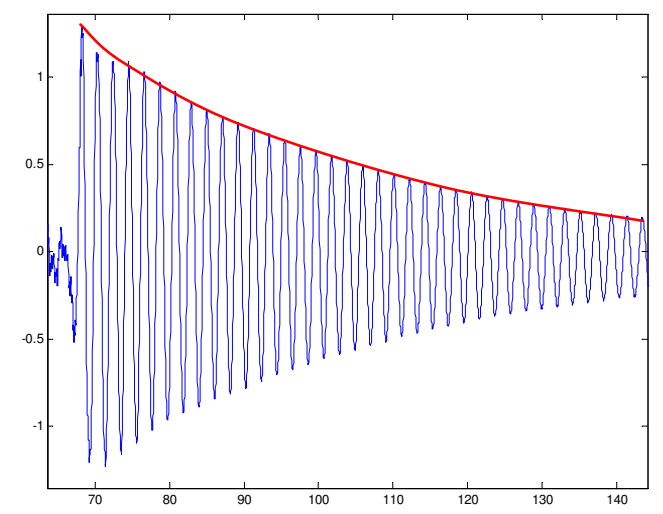

Figure 6.3 Free vibration in the first mode due to emergency braking, with fitted amplitude decay trend line [53]

From the measurement, the first natural frequency can be determined. A very simple and straightforward approach is to take a certain duration from the measurement, count all zero crossings and divide the number of cycles by the duration. In this case the total number of zero crossings is 97 , so the number of full cycles $(n-1) / 2=48$. The duration was $100 \mathrm{~s}$, so the natural frequency is $0.48 \mathrm{~Hz}$.

The damping can be determined using the half-amplitude method shown in Figure 6.4. If the reduction of displacement from point $P$ to point $R$ is $50 \%\left(\hat{x}_{R}=0.5 \hat{x}_{P}\right)$ and the amplitude envelope curve is described by:

$$
\hat{x}(t)=\frac{\hat{x}_{0}}{\sqrt{1-\beta^{2}}} e^{-\beta 2 \pi f_{n a t} t}
$$

with:

\begin{tabular}{l|l|l}
\hline$\hat{x}_{0}$ & amplitude & {$[\mathrm{m}]$} \\
$\beta=c / c_{c r}$ & fraction of critical damping & {$[-]$} \\
$f_{\text {nat }}$ & undamped natural frequency & {$[\mathrm{Hz}]$} \\
$t$ & time & {$[\mathrm{s}]$}
\end{tabular}

The damping can be calculated with: 


$$
\frac{\hat{x}_{P}}{\hat{x}_{R}}=\frac{\hat{x}\left(t_{2}\right)}{\hat{x}\left(t_{1}\right)}=2=e^{-\beta 2 \pi f_{\text {nat }}\left(t_{2}-t_{1}\right)}
$$

Using the measured data, the damping is found to be $\beta=0.0095$ or $0.95 \%$ of the critical damping. This confirms the recommended design value of $1 \%$ in table 6.2.

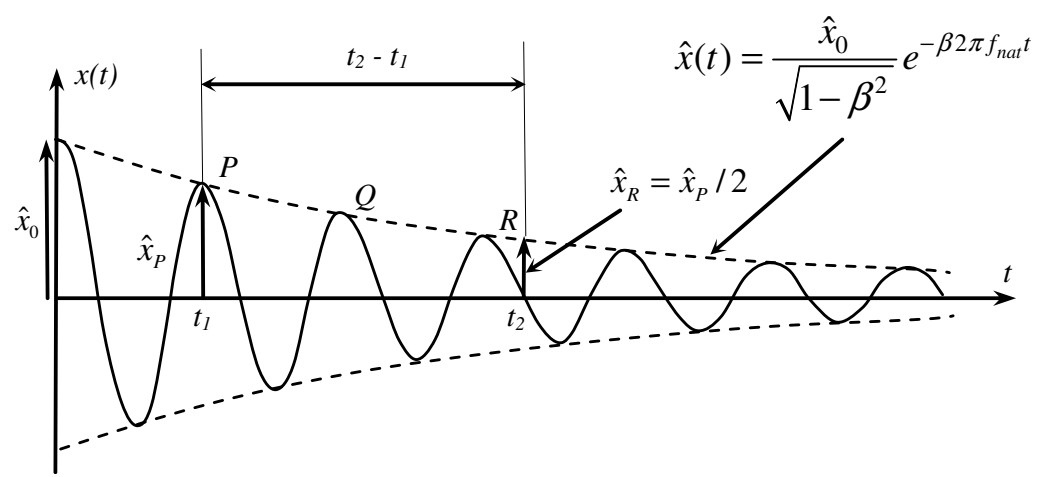

Figure 6.4 Half-amplitude method for determining the damping factor

\subsubsection{Simulation parameter setting for time and frequency domain methods}

As all analyses will be carried out in, or will be transformed to, the frequency domain, the main interest of setting the simulation parameters is to make sure that all relevant information is sampled in sufficient detail in both time and frequency domain descriptions. The characteristics of the operating turbine that will have an impact on the analysis of the bending stress in the support structure are summarized in table 6.3.

Table 6.3 Frequencies of interest for support structure bending stress

\begin{tabular}{l|l} 
range of turbulent wind frequencies & $1 / 600 \mathrm{~Hz}-1 \mathrm{~Hz}(10 \mathrm{~min}$ duration $)$ \\
range of wave frequencies at Blyth & $0.05-0.5 \mathrm{~Hz}$ \\
first natural frequency & $0.47 \mathrm{~Hz}$ \\
1P, rotational frequency & $0.175-0.36 \mathrm{~Hz}$ \\
3P, blade passing frequency & $0.525-1.08 \mathrm{~Hz}$
\end{tabular}

To have a safe margin on the highest relevant frequency of interest of 3P at $1.08 \mathrm{~Hz}$ and to prevent aliasing, this highest required sample frequency is set at $1.5 \mathrm{~Hz}$. As discussed in section 2.3, the Nyquist frequency is then determined by:

$$
f_{\text {Nyquist }}=(4 / 3) f_{\max }=2 \mathrm{~Hz}
$$

and corresponding time step in the time domain will be: 


$$
\Delta t=1 /\left(2 f_{\text {nyquist }}\right)=0.25 \mathrm{~s}
$$

The required frequency resolution and associated subrecord length are determined jointly by the minimum frequency required to model a 10 minute turbulent wind field and by the first natural frequency of the support structure (see Figure 2.7). The support structure is very lightly damped, resulting in a very sharp resonance peak. To cover this sharp peak with sufficient frequency steps, the frequency step must be chosen smaller than the half power bandwidth, as explained in section 2.3. With a natural frequency of $f_{\text {nat }}=0.47 \mathrm{~Hz}$ and a damping factor of $\beta=0.01$, the required frequency step is determined by:

$$
\Delta f=1 / 2 \beta f_{\text {nat }}=2.35 \cdot 10^{-3} \mathrm{~Hz}
$$

With this required frequency step, the minimum required subrecord length is found:

$$
T_{r}=1 / \Delta f=425.5 \mathrm{~s}
$$

while to cover the minimum frequency $f_{\min }$ in the turbulent wind field, the minimum required subrecord length is:

$$
T_{r}=1 / f_{\min }=600 \mathrm{~s}
$$

The time domain data is converted to spectra through FFT. The subrecord lengths needed for FFT are dependent on 2 to the power $N N$ :

$$
T_{r-F F T}=2^{N N}(\Delta t)=2^{N N}(0.25)
$$

so for:

$$
N N=11 \rightarrow T_{r-F F T}=2048 \cdot 0.25=512 \mathrm{~s}
$$

This is close enough to $425.5 \mathrm{~s}$ and $600 \mathrm{~s}$. However, a single time domain simulation is only one of an infinite number of possible realisations. Processing of time domain data therefore requires a considerable number of simulations before statistically reliable results can be obtained. All these simulations should be subjected to FFT. By averaging the results, reliable response spectra and transfer functions can be determined. For good results [54] advises to use a minimum of 50 records, preferably 100 or even 200 records. By applying a $50 \%$ overlap of the subrecords, only $50 \times T_{r}$ is required to still 
have a total of 100 records for averaging. This means that the total required time series length follows from:

$$
T_{\text {total }}=50 \times T_{r-F F T}=25600 \mathrm{~s}=7.1 \text { hours }
$$

\subsubsection{Influence of time step on the simulations and their analysis}

The simulation settings presented in the previous section were selected primarily to facilitate frequency domain processing of time domain data. During the first simulations however, the time step of $0.25 \mathrm{~s}$ turned out to be too large for the internal simulation process within Bladed. In the full length time domain simulation, used as a reference for the frequency domain method, anomalous energy showed up in the area between $0.6 \mathrm{~Hz}$ and $0.9 \mathrm{~Hz}$ (Figure 6.5). This energy could not be explained: both excitation frequencies and structural characteristics do not justify any response energy in that frequency range. Furthermore, neither the frequency domain method nor in the measurements from the real offshore wind turbine at Blyth revealed such behaviour.

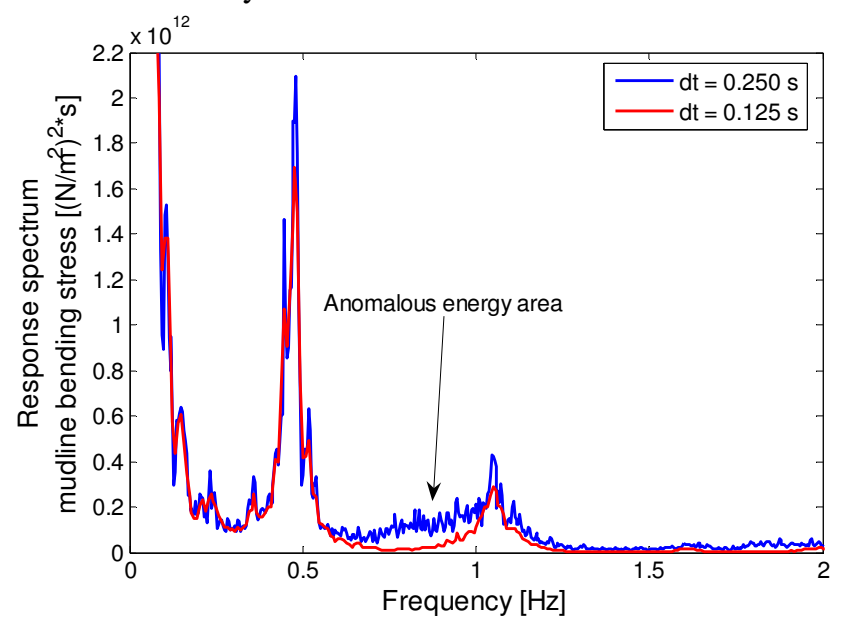

Figure 6.5 Response spectra for $V_{w}=10 \mathrm{~m} / \mathrm{s}$ with anomalous energy area

A series of simulations with different structural characteristics (stiffer and softer tower, no blade dynamics) did have some effect, but could not explain the anomaly. Only when the time step was reduced to $0.125 \mathrm{~s}$ did the anomalous energy vanish. Figure 6.6 shows the response spectra for mudline bending stress for a number of different time steps. The anomalous energy disappeared from the area of interest at a time step of less than $0.14 \mathrm{~s}$. The peak turns out to be an aliasing effect as described in section 2.3.5. Because Bladed uses a smaller internal time step than the output time step, the signal does contain higher order frequencies, which are picked up by the FFT process. 


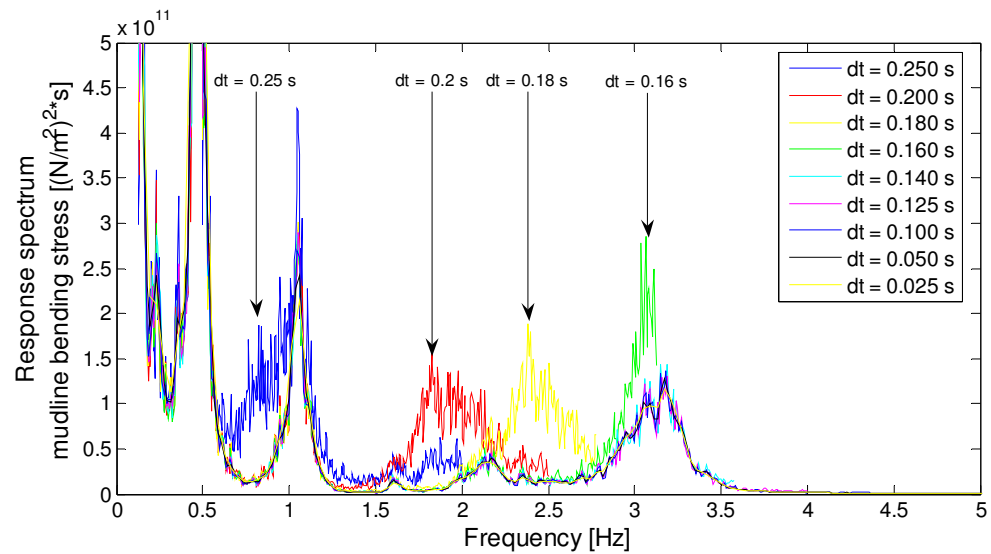

Figure 6.6 Response spectra for $V_{w}=10 \mathrm{~m} / \mathrm{s}$ for different time steps

Due to the smaller time step, the cut-off frequency shifted to the right, revealing a peak at $3 \mathrm{~Hz}$ feeding the aliasing effect: the second natural frequency of the support structure. The initial frequency range selected for the analysis did not include this frequency, but the peak does seem relatively large. However, a simple estimate of the impact of the peak showed that it is completely irrelevant. The area under the peak at the second natural frequency, which is equal to its contribution to the zeroth order spectral moment, is:

$$
\Delta m_{0}=0.75 *\left(2.10^{11}\right) * 0.5=75 \cdot 10^{9} \mathrm{~N} / \mathrm{m}^{2}
$$

The contribution to the standard deviation of the bending stress is the square root of this area, which amounts to about $0.27 \mathrm{MPa}$ and is by any means negligible.

The conclusion that can be drawn from these trial simulations is that a time step change is such that internal processes of the simulation software do not give erroneous output. The new simulation parameters, resulting from this reduced time step size, are summarized in table 6.4.

Table 6.4 New simulation settings for smaller time step

\begin{tabular}{l|l|l}
\hline$\Delta t$ & 0.125 & {$[\mathrm{~s}]$} \\
$f_{\text {Nyquist }}$ & 4 & {$[\mathrm{~Hz}]$} \\
$T_{r}$ (unchanged) & 600 & {$[\mathrm{~s}]$} \\
$N N$ & 12 & {$[-]$} \\
$T_{r-F F T}$ & 512 & {$[\mathrm{~s}]$} \\
$T_{\text {total }}$ & 7.1 & {$[$ hours $]$} \\
number of sub-records for averaging & 100 & {$[-]$} \\
overlap & 50 & {$[\%]$} \\
windowing technique & Hanning & {$[-]$}
\end{tabular}




\subsubsection{Defining the aerodynamic environment}

For all simulations, the wind climate as defined by Germanischer Lloyd [20] has been used. GL prescribes a fixed turbulence intensity of $12 \%$ for all wind speed classes. Although higher turbulence intensity at lower wind speeds, as prescribed by other standards and shown in section 2.5, represents nature better, for the validation of the frequency domain method a fixed turbulence intensity is convenient and sufficient. For the wind shear the same standard was used, giving a shear factor $\alpha=0.12$. This is again used for all wind speeds. Waves and currents are not included in these wind simulations.

\subsection{Transfer function for fluctuating wind speed to tower top load}

First, a 3D turbulence field is modelled based on an improved Von Kármán spectrum for a mean wind speed of $10 \mathrm{~m} / \mathrm{s}$ and a turbulence intensity of $12 \%$ in longitudinal direction. Then a simulation is carried out for the offshore wind turbine model at Blyth. The modal analysis of the support structure is set to not incorporate any modes, which effectively eliminates support structure dynamics. The blade modes are still active.

After the simulation, a spectrum estimate is determined for the wind speed at the hub. This results in the spectrum shown in Figure 6.7, when plotted on log-linear scale.

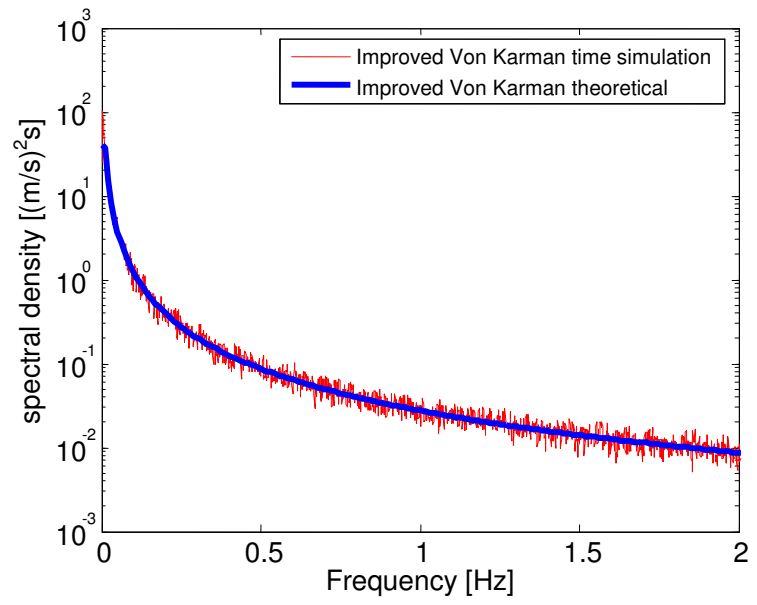

Figure 6.7 Wind spectrum, mean: $10 \mathrm{~m} / \mathrm{s}$, turbulence intensity: $12 \%$

For the resulting tower top load, the response spectrum is obtained by processing the time domain simulation of the axial load on the rotor axis using the same spectral settings; the result is presented in Figure 6.8. The effects of rotational sampling at 3P, $6 \mathrm{P}$ and $9 \mathrm{P}$ are clearly visible. 


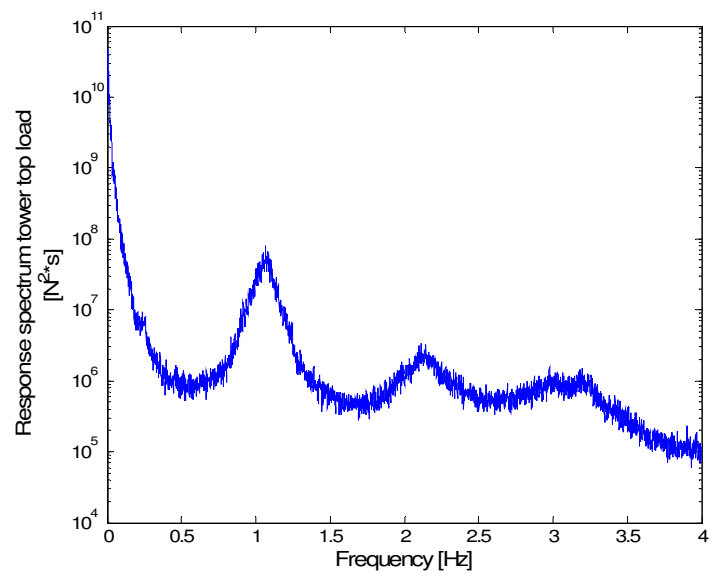

Figure 6.8 Response spectrum for tower top load

Now, the transfer function can be determined by taking the square root of the response spectrum divided by the wind spectrum at each frequency. Because the spectral estimates have been created with the same settings, the frequency intervals are the same, making the calculation of the transfer function very straightforward.

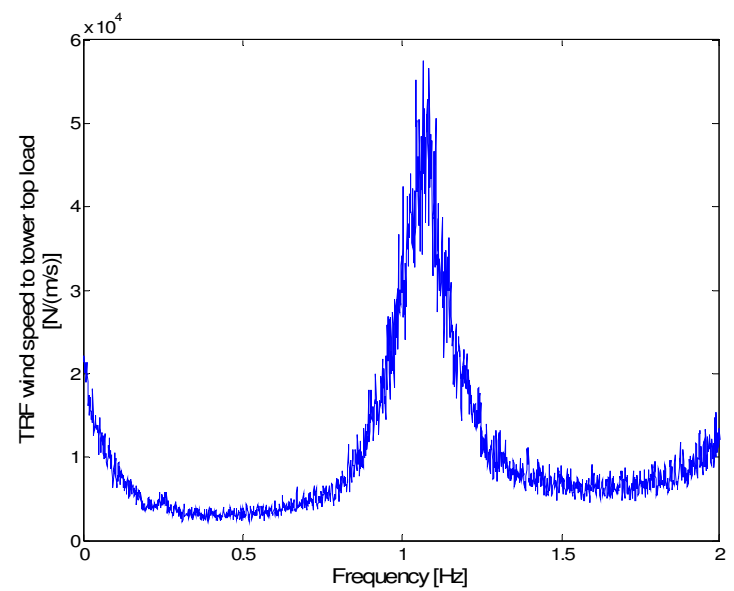

Figure 6.9 Transfer function between wind speed and tower top load

The mean rotor speed in this simulation is $21 \mathrm{RPM}=0.35 \mathrm{~Hz}$. The $1 \mathrm{P}$ peak cannot be distinguished, the $3 \mathrm{P}$ peak at $1.05 \mathrm{~Hz}$ is clearly visible. It has to be noted that the $1^{\text {st }}$ blade flapping frequency also lies in this range, which is, based on the "Campbelldiagram" approach [21], not entirely an ideal situation. 


\subsection{Incorporating aerodynamic damping}

As described in section 5.3.2, the aerodynamic damping can be calculated through different methods. Here only three of them are applied:

- Garrad

- non-linear simulation

- engineering estimate.

The aerodynamic damping was found to be as shown in Figure 6.10. The engineering estimate is equal to the value deduced for the Blyth offshore wind turbine by Garrad Hassan and Partners in the OWTES report [47]: an aerodynamic damping of $4 \%$.

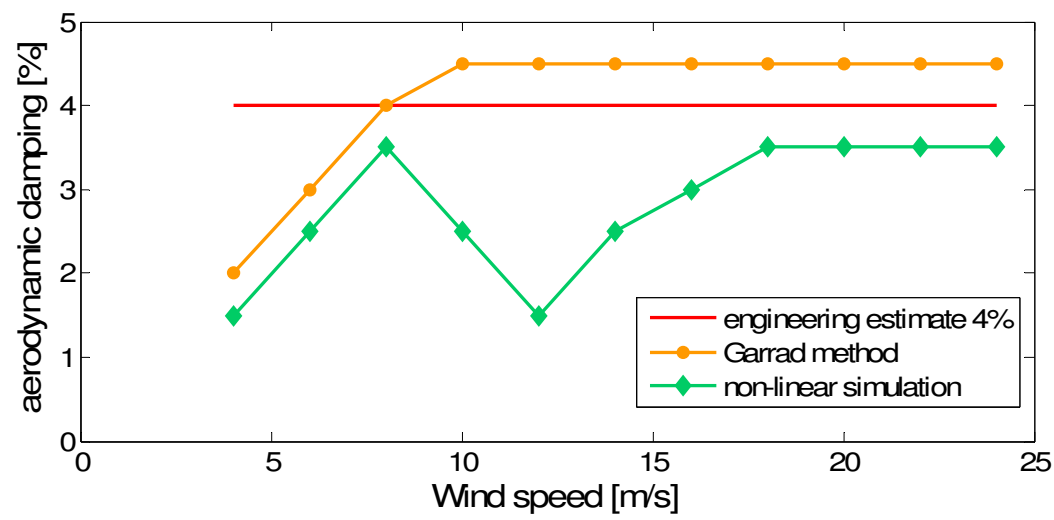

Figure 6.10 Aerodynamic damping as function of wind speed in percentage of critical damping for the Garrad method (orange, bullets), the non-linear simulation method (green) [80] and the engineering estimate (red)

Figure 6.10 shows that the engineering estimate is a little higher than the theorical aerodynamic damping originating from the Garrad method. The non-linear simulation method results in a lower damping for higher wind speeds. The conclusions from this in [80] are that the pitch control modelling in the simulation tool seems to have a large effect on the damping above rated wind speed. For this chapter it was found that the engineering estimate of $4 \%$ suits these calculations well. It is noted that the aerodynamic damping needs to be studied more closely for better understanding and modelling. Chapter 7 will also incorporate different estimates of aerodynamic damping.

\subsection{Modelling the support structure}

The finite element program SESAM was used to derive the transfer function for tower top load to mudline bending stress. A model of the offshore wind turbine was made and the natural frequency in SESAM was found to compare very well with the measured 
frequency and the frequency as modelled in Bladed. The structural damping was set to $1 \%$ and to $(1 \%+4 \%=) 5 \%$ of the critical damping, for situations without and with aerodynamic damping, respectively. A tower top load of $1000 \mathrm{~N}$ was applied with increasing frequency from $0.01 \mathrm{~Hz}$ to $2 \mathrm{~Hz}$ in steps of $0.001 \mathrm{~Hz}$, resulting in the transfer functions shown in Figure 6.11. The effect of incorporating the aerodynamic damping is clearly very large.

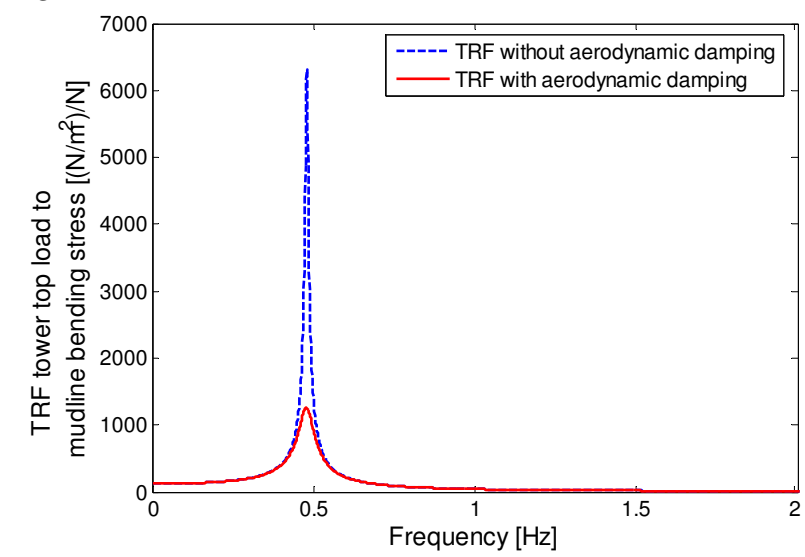

Figure 6.11 Transfer function of mudline bending stress per unit tower top load as function of frequency with only structural damping (1\%) and additional aerodynamic damping (4\%)

By combining the transfer functions of Figure 6.9 and Figure 6.11, the combined transfer function is derived, Figure 6.12.

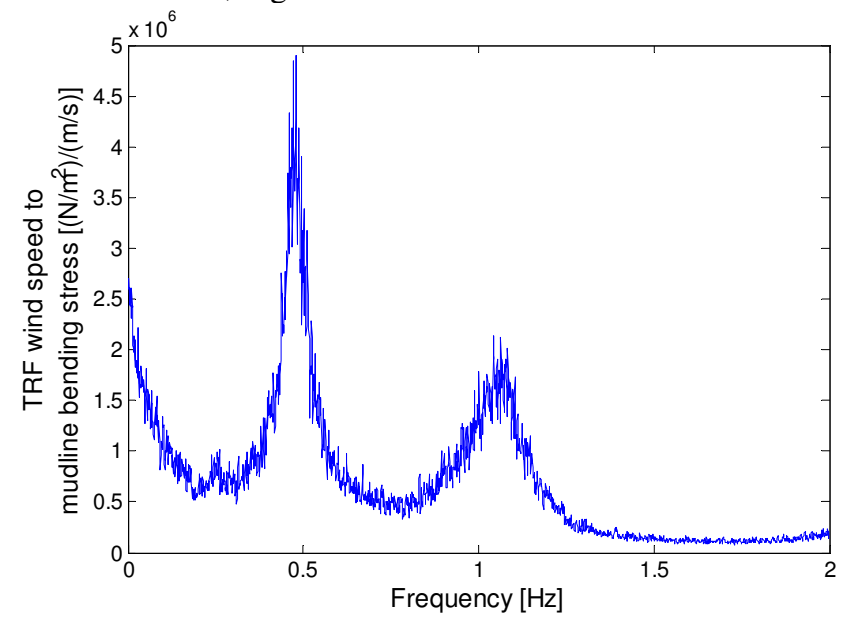

Figure 6.12 Combined transfer function of mudline bending stress per unit wind speed as function of frequency, including aerodynamic damping 
By multiplying the square of the combined transfer function with the input wind spectrum, the response spectrum for bending stress at the mudline can be determined, as shown in Figure 6.13. In the time domain, the time series of the mudline bending stress for the same conditions can be found by using a full dynamic model of the offshore wind turbine (turbine and support structure). The resulting time series of the varying bending stress can be transformed to a spectrum through FFT. This spectrum is also shown in Figure 6.13.

Figure 6.13 shows that the results match very well. The shapes are identical and only the peak at $1 \mathrm{~Hz}$, which corresponds with both the 3P-blade passing frequency and the first blade flap frequency, is slightly lower when calculated in the frequency domain. Apparently the overlapping of these frequencies influences the support structure when all dynamics are modelled in the time domain.

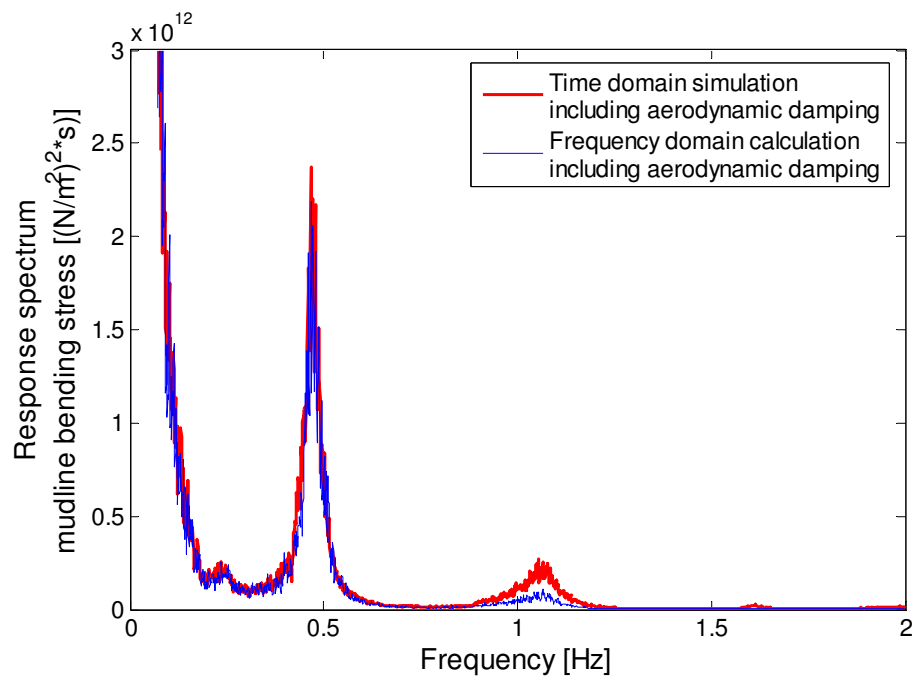

Figure 6.13 Mudline bending stress response spectra for frequency and time domain calculations

However, similar as for the peak at the second natural frequency of the support structure at $3 \mathrm{~Hz}$ in section 6.2.3, the contribution to the variable bending stress from the peak at $1 \mathrm{~Hz}$ is completely irrelevant. The contribution to the standard deviation of the bending stress is approximately:

$$
\Delta \sigma_{s} \approx \sqrt{(0.35) *\left(0.2 \cdot 10^{12}\right) *(0.5)}=\sqrt{3.5 \cdot 10^{10}}=0.19 \cdot 10^{6} \mathrm{~N} / \mathrm{m}^{2}=0.19 \mathrm{MPa}
$$

which is negligible, both as an absolute stress value and in comparison to the other peaks in the spectrum. 


\subsection{Comparison of frequency domain calculations with time domain calculations and with measurements under wind loading only}

\subsubsection{Simulations for wind speeds of 6-20 m/s}

All previous simulations were based on $10 \mathrm{~m} / \mathrm{s}$ wind, this to be able to compare different parameter settings. Because a wind turbine responds non-linearly to different mean wind speeds, simulations for several wind speeds are necessary to evaluate the applicability of the frequency domain method for the entire design scope. Steps of $2 \mathrm{~m} / \mathrm{s}$ mean wind speed cases were used from $6 \mathrm{~m} / \mathrm{s}$ to $20 \mathrm{~m} / \mathrm{s}$ wind speed. For all cases a turbulence intensity of $12 \%$ was taken, as per Germanischer Lloyd for offshore wind turbine design [20]. As before, for all simulations the same length of $7.1 \mathrm{~h}$ with a time step of $0.125 \mathrm{~s}$ was used. The time series were sampled at $2^{12}=4096$ points per subrecord with Hanning windowing and 50\% overlap. Figure 6.14 shows 4 cases for increasing wind speed, with spectra obtained from the full time domain simulation and directly from the frequency domain method. The $x$ and $y$ axis scales are the same for all 4 plots. It can be seen that the results for both methods match very well. For higher wind speeds, increasingly more energy reaches the support structure's natural frequency at $0.47 \mathrm{~Hz}$ giving a growing resonance peak. The $3 \mathrm{P}$ peak can be seen to shift to the right from $6 \mathrm{~m} / \mathrm{s}$ wind to $8 \mathrm{~m} / \mathrm{s}$ wind as the rotation speed increases from 16 RPM to 21 RPM (1P at $0.27 \mathrm{~Hz}$ and $0.35 \mathrm{~Hz}, 3 \mathrm{P}$ at $0.8 \mathrm{~Hz}$ and $1.05 \mathrm{~Hz}$ ).
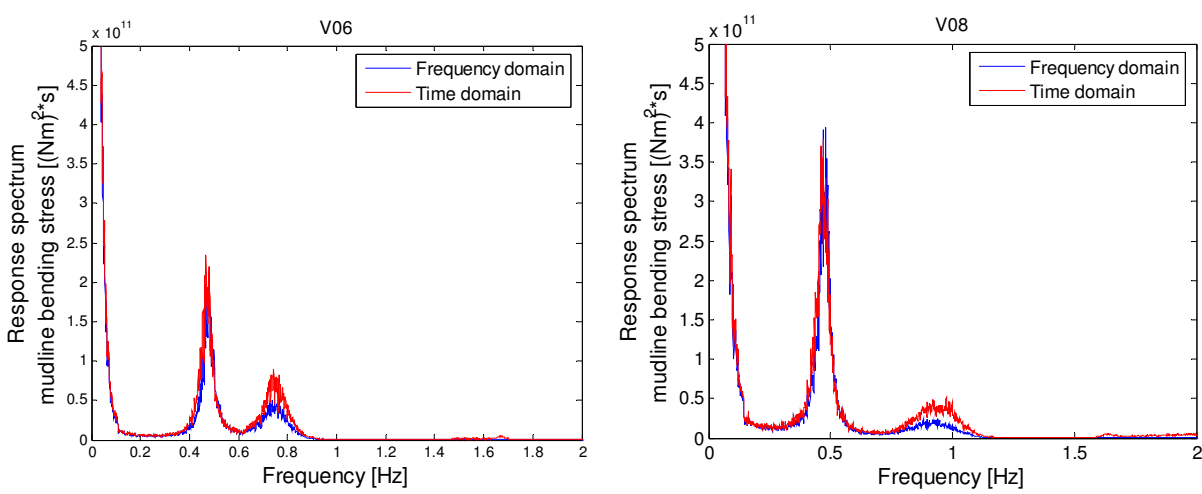

${ }^{\circ}$ The simulation length per wind speed class is over 7 hours to acquire a statistically stable transfer function. These simulations only have to be perfomed once for each turbine type and can than be re-used infinitely for support structure design. 

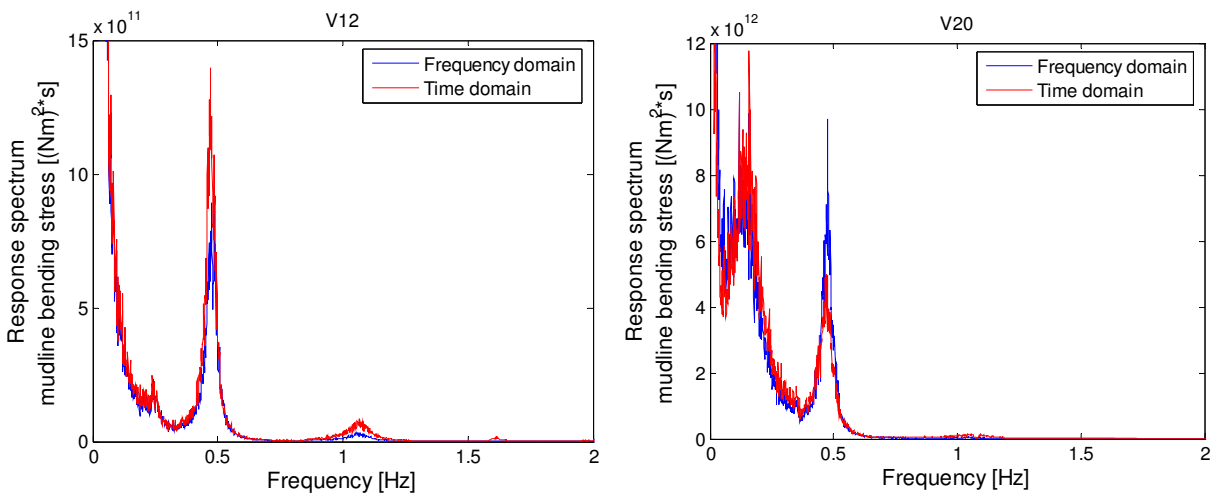

Figure 6.14 Response spectra of mudline bending stress from frequency domain and full time domain calculations for $6,8,12$ and $20 \mathrm{~m} / \mathrm{s}$ wind speed

\subsubsection{Comparison of time domain results with measurements at Blyth}

A final comparison can be made with measurements from the actual offshore wind turbine at Blyth. The biggest problem in this respect is the constant presence of waves at this location, however small. Review of all 580 campaigns of 30 minutes length resulted in only 2 usable cases when the turbine is in operation and the waves are nearly absent $\left(H_{s}<0.6 \mathrm{~m}\right)$. Table 6.5 gives an overview of the parameters for these two campaigns. Figure 6.15 shows the mudline bending moment spectra for these two cases, together with spectra from the time domain simulations. Time domain simulations were used here because the previous sections have shown that frequency and time domain method produce nearly equal spectra, but, for this comparison, tuning all parameters to match the measurements was done more easily in the time domain.

Table 6.5 Campaign parameters for $\mathrm{x} 00 \mathrm{e} 9$ and $\mathrm{x} 0660$

\begin{tabular}{c|c|c} 
campaign code & x00e9 & x0660 \\
\hline \hline date and time & 27 Oct $2001.13: 12: 32$ & $14 \mathrm{Jan} 2003.15: 05: 57$ \\
$V_{w}$ & $11.77 \mathrm{~m} / \mathrm{s}$ & $15.34 \mathrm{~m} / \mathrm{s}$ \\
$\sigma_{v}$ & $2.41 \mathrm{~m} / \mathrm{s}$ & $2.81 \mathrm{~m} / \mathrm{s}$ \\
$I_{t}$ & $20 \%$ & $18 \%$ \\
$V_{d i r}$ & $267^{\circ}$ & $243^{\circ}$ \\
Mean power & $1363 \mathrm{~kW}$ & $1952 \mathrm{~kW}$ \\
$H_{s}$ & $0.45 \mathrm{~m}$ & $0.59 \mathrm{~m}$ \\
$T_{p}$ & $5.5 \mathrm{~s}$ & $9.8 \mathrm{~s}$
\end{tabular}



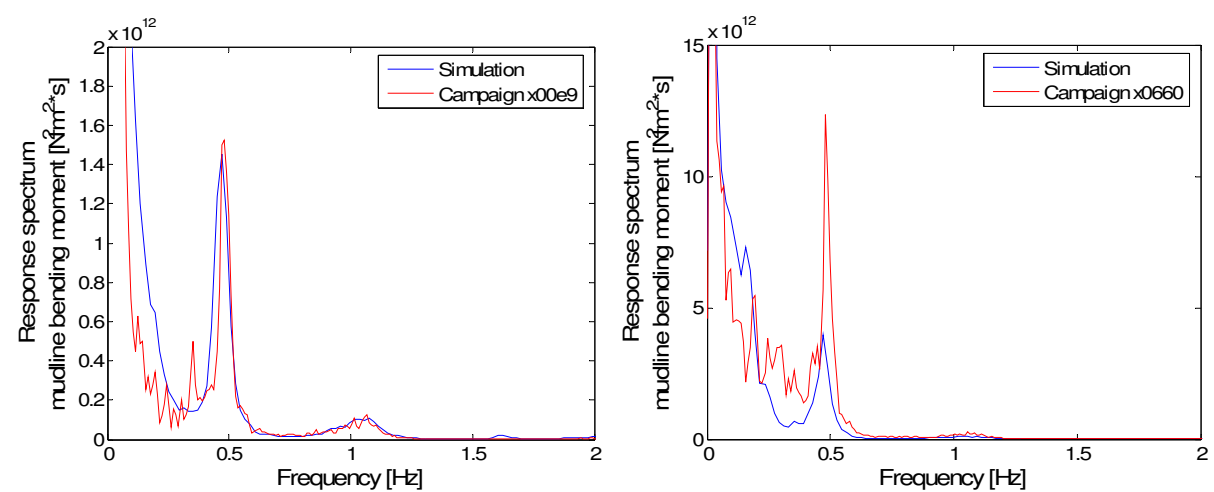

Figure 6.15 Comparison between measurement x00e 9 and x0660 with the time domain method for wind loading only

The following observations can be made from the graphs in Figure 6.15:

- The turbulence noted in table 6.3 was used as input for Bladed to simulate the wind field, but this value is measured on the nacelle, behind the rotor; therefore the actual turbulence may well be lower. Moreover, the actual turbulence spectrum chosen for the time simulation was a Von Kármán spectrum, whereas the actual spectral shape may be different.

- The left figure (x00e9) shows a very nice match with only slight deviation of the low frequency part, which is probably due to the differences between the model and reality as mentioned in the first bullet

- In the right figure (x0660), the influence of waves is clearly present in the measurements, introducing additional energy between 0.2 and $0.5 \mathrm{~Hz}$. This explains the difference between the graphs in that area, including the significantly higher resonance peak in the measurements.

- Taking the uncertainties of the measured data and the random error in the measured spectra due to the short duration $(30 \mathrm{~min})$ of the measurements campaigns into account, the response spectra from the time domain simulations for the nearly wind-only load cases show very satisfactory agreement with the measurements. This demonstrates that the wind field and structural models, as well as the Bladed program are giving sattisfactory results. 


\subsection{Comparison of frequency domain results with time domain and measurements under combined wind and wave loading}

\subsubsection{Introduction}

After the analysis of the offshore wind turbine subjected to wind loading only, now the response due to wave loading is added. The method of combining wind and wave response is very straightforward: the response spectra due to wind loading and due to wave loading are created independently and in such a way that the frequency steps are equal. Then the ordinates of both spectra are simply added. First, section 6.7.2 presents the 5 reference cases used for the comparison.

\subsubsection{Reference load cases}

Within the OWTES task 4.3, robustness of design calculations [53], the site data at Blyth was analysed to facilitate fatigue damage calculations. A detailed report has been made available to the project, describing the environment in terms of simultaneous wind and wave conditions. To reduce the number of calculations, the data was binned into a three-dimensional scatter diagram giving the joint probability of significant wave height, wave zero-crossing period and mean wind speed. This diagram was further reduced to 15 characteristic environmental states, as summarized in table 6.6.

Table 6.6 Summary of 15 environmental states for Blyth [53]; the 5 states for testing the frequency domain method against the time domain method and a corresponding measurement

\begin{tabular}{|c|c|c|c|c|c|c|}
\hline & $\begin{array}{l}H_{s} \\
(\mathrm{~m})\end{array}$ & $\begin{array}{l}T_{z} \\
(\mathrm{~s})\end{array}$ & $\begin{array}{c}V_{w} \\
(\mathrm{~m} / \mathrm{s})\end{array}$ & $\begin{array}{c}\% \text { of } \\
\text { occurence }\end{array}$ & campaign & $\begin{array}{c}\text { demonstration } \\
\text { case }\end{array}$ \\
\hline 1 & "0.25 & 2.0 & 5.0 & 20.47 & none & 1 \\
\hline 2 & 0.25 & 5.2 & 4.9 & 3.73 & & \\
\hline 3 & 0.25 & 4.0 & 11.8 & 21.76 & $\mathrm{x} 00 \mathrm{e} 9$ & 2 \\
\hline 4 & 0.25 & 5.6 & 15.7 & 3.85 & & \\
\hline 5 & 0.25 & 5.8 & 20.6 & 1.00 & & \\
\hline 6 & 0.75 & 3.4 & 6.7 & 8.62 & & \\
\hline 7 & 0.75 & 5.3 & 5.8 & 13.25 & x00da & 3 \\
\hline 8 & 0.75 & 5.5 & 11.7 & 5.58 & & \\
\hline 9 & 1.25 & 5.2 & 8.8 & 10.66 & & \\
\hline 10 & 1.25 & 8.0 & 8.5 & 1.25 & & \\
\hline 11 & 1.75 & 6.0 & 9.9 & 4.83 & $\mathrm{x} 051 \mathrm{e}$ & 4 \\
\hline 12 & 1.75 & 6.7 & 16.2 & 0.55 & & \\
\hline 13 & 2.4 & 6.8 & 12.8 & 3.54 & & \\
\hline 14 & 3.4 & 7.8 & 14.5 & 0.77 & & \\
\hline 15 & 3.3 & 9.7 & 18.7 & 0.14 & x0581 & 5 \\
\hline
\end{tabular}


In the table, the 5 demonstration cases that will be used for the comparison between the time domain and the frequency domain method are highlighted. A corresponding measurement campaign hs been selected with an environment that is nearly equal to the selected demonstration case. For case 1 no such campaign is available; the computer at Blyth was activated to record only specific cases, small waves and light wind were not among these. Comparison with the measurements will be reported in section 6.7.4.

\subsubsection{Comparison of frequency domain and time domain calculations}

The SESAM model of the support structure used in section 6.5 to derive the transfer function for tower top load to bending stress was also used to determine the transfer function for wave loading on the support structure. The hydrodynamic parameters are defined and 60 frequency steps are selected for which the transfer function will be determined. These frequency steps are chosen in such a way that they cover the range of interest for wave loads; i.e. from $0.05 \mathrm{~Hz}$ to $1 \mathrm{~Hz}$. Furthermore, the frequency steps are very finely distributed in the area of the peak of the wave spectrum and around the natural frequency of the offshore wind turbine.

The damping of the model was again set to $5 \%$ of critical damping, which incorporates the $4 \%$ aerodynamic damping. The first 25 modes were used to model structural dynamics combined with static back substitution ${ }^{1}$ to achieve a fully correct quasi-static structural response. Waves with the same frequency as the natural frequency of the offshore wind turbine $(0.47 \mathrm{~Hz})$ have a length of only $7.1 \mathrm{~m}$, which is approximately twice the diameter of the foundation pile and transition piece on which the waves act. Therefore wave diffraction, as described in section 2.4.3, will have considerable influence on the wave loads.

In the first run with SESAM, the diffraction correction was not incorporated. The resulting transfer function is shown in Figure 6.16. Figure 6.16 also shows the transfer function including the correction; the impact for all frequencies above approximately $0.3 \mathrm{~Hz}$ is very significant and the resonance peak nearly disappears. The transfer function corrected for the effects of wave diffraction has been used in further calculations.

\footnotetext{
${ }^{1}$ In practical applications modal analysis of a finite element model only solves a limited number of mode shapes. The higher modes are discarded, which is no problem for the dynamic solution as all high modes do not add significantly to the dynamic response. However, as the (quasi-) static response is also a part of the complete response, all static contributions from the higher modes are also lost. This causes major deviations in stress calculations. Two solutions exist: either solve all modes for the finite element model or remove the static components from the limited number of modes, calculate the full static solution and add that to the dynamic solution from the limited number of modes. This last option is called "static back substitution".
} 


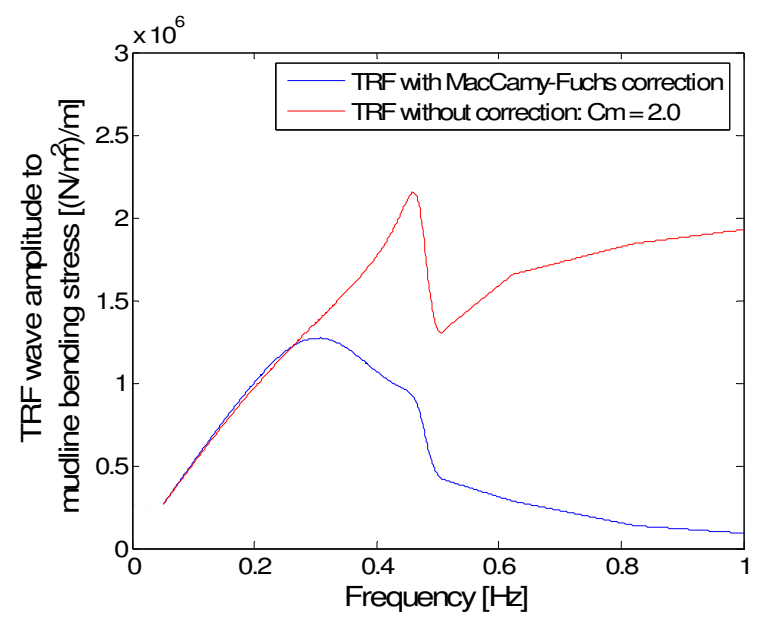

Figure 6.16 Transfer function of mudline bending stress per unit wave amplitude with and without MacCamy-Fuchs diffraction correction

Combining the response spectra for mudline bending stress due to wind and wave loading was done in Excel. To be able to use the wind speeds in section 6.6.1 with steps of $2 \mathrm{~m} / \mathrm{s}$, the wind speed settings of the cases highlighted in table 6.6 were rounded to fit these wind speeds. The resulting spectra are compared with spectra determined from time domain simulations in Bladed for the same conditions. The Bladed simulations were run with a length of $3600 \mathrm{~s}$, i.e. 7 subrecord lengths of $512 \mathrm{~s}$. The time series were FFTed to a spectrum using 4096 points per subrecord, including Hanning windowing and a $50 \%$ overlap; this provides for 14 spectral averages. Due to this relatively low number of averages the spectra from the time domain simulations show more random error (are less smooth) than the spectra from the frequency domain calculations. Figure 6.17 shows the spectra for the 5 cases. Note that not all the vertical scales of the figures are the same. 

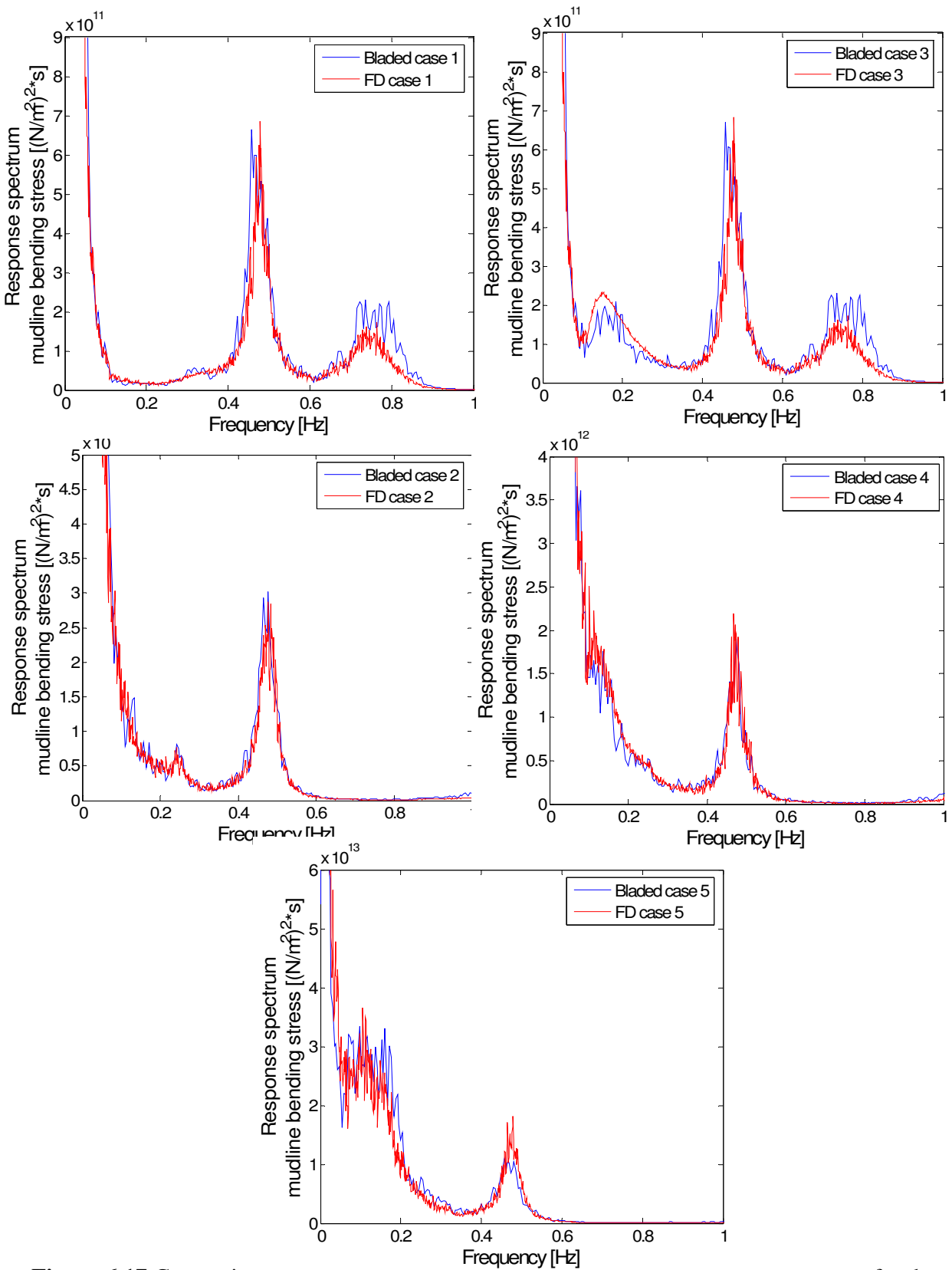

Figure 6.17 Comparison between trequency domain and tıme domain response spectra for the mudline bending stress for 5 wind and wave load cases 
The first and most important conclusion to be drawn from these figures is that the agreement between the results of both methods is striking. The stress response spectra compare very well, proving that the frequency domain method is a fully acceptable alternative to time domain simulation. From cases 1, 2 and 3 it becomes clear that the turbulent wind has a large influence on this particular offshore wind turbine configuration. Structural resonance (the peak around $0.47 \mathrm{~Hz}$ ) is a significant contributor to the mudline bending stress and is due to wind excitation only; the wave induced stress is only of minor importance for this structure. This was to be expected due to the very shallow water and high first natural frequency of the offshore wind turbine. For cases 4 and 5 the wave conditions are more severe and therefore become relatively more important. The quasi-static response to the wave spectrum increases and becomes more important than the resonance contribution, as can be clearly seen in cases 4 and 5 .

The blade passing frequency $3 \mathrm{P}$ is only visible in cases 1 and 3 around $0.75 \mathrm{~Hz}$. For these cases the $3 \mathrm{P}$ frequency is lower than for the other cases where the turbine runs at maximum speed of $21 \mathrm{RPM}, 0.35 \mathrm{~Hz}$ and a $3 \mathrm{P}$ frequency of $1.05 \mathrm{~Hz}$. The relative impact of the $3 \mathrm{P}$ frequency is only significant for these lower wind speed cases 1 and 3.

\subsubsection{Comparison of spectra from frequency domain calculations with spectra from measurements at Blyth}

To complete the comparisons for the offshore wind turbines at Blyth, the response spectra found with the frequency domain method are compared with spectra obtained from the measurement campaigns for cases 2 to 5 mentioned in table 6.6. Figure 6.18 shows the results.
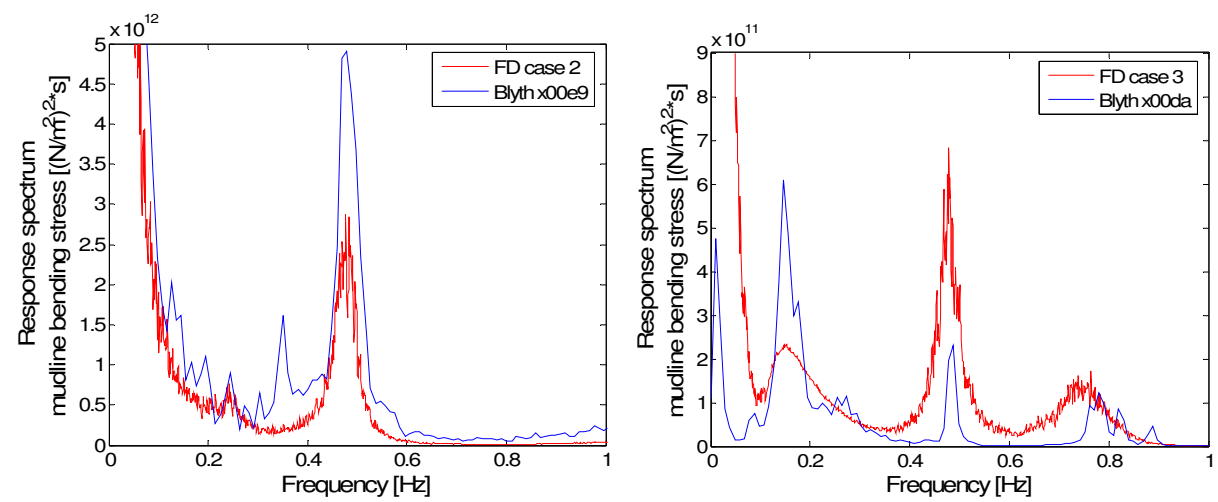

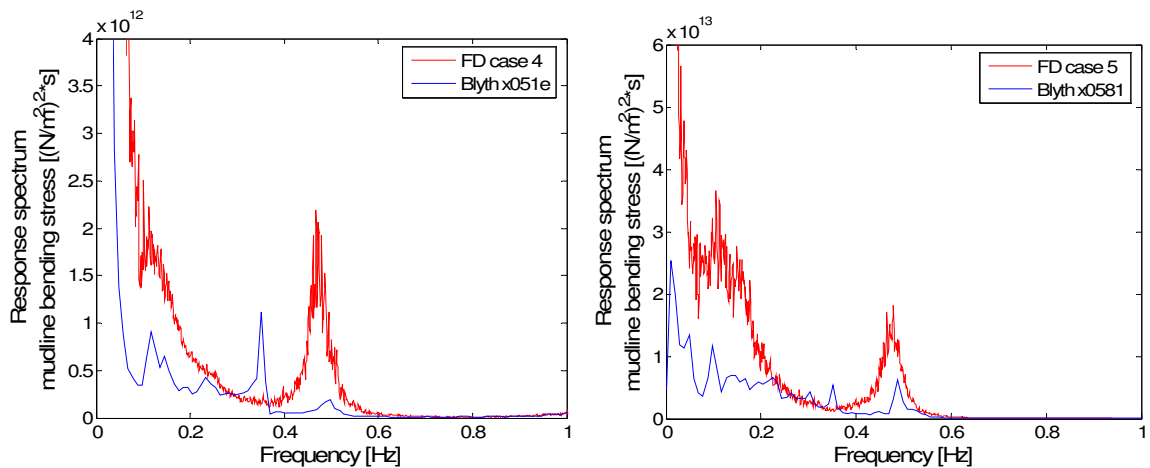

Figure 6.18 Comparison of frequency domain response spectra for mudline bending stress with measured spectra for combined wind and wave loading

When comparing full-scale measurements with theoretical calculations one should always be aware of relevant differences:

- Conditions during the full-scale measurements can generally not be controlled. This means, for example, that the real environment during the measurements is generally not exactly the same as the environment modelled in the theoretical calculations. This applies to the wind fields, but in the present situation even more so to the sea state. The Blyth location is close to the coast, so that the wind fetch for different wind directions is quite variable. Turbulence spectra will also deviate from the actual on-site turbulence. Combined with the shallow water depth and complex bathymetry it is unlikely that the actual, multidirectional sea state generated by local winds is well represented in a theoretical, unidirectional Pierson-Moskovitz spectrum.

- Similar considerations apply to differences between the actual structure and its representation in the theoretical model, including foundation behaviour. In the present situation this is considered to be of minor influence, however.

- Natural excitation is three-dimensional and due to all sources, however small, while the theoretical model is often purposely limited to describing twodimensional behaviour and the effect of one source of excitation only. The measured spectra are therefore likely corrupted, to some extent, by other excitations and by three-dimensional effects. The observations made in the first point about multidirectional sea states and their unidirectional representation in the theoretical model is very exemplary in this respect.

- Measurement durations are unavoidably limited, e.g. due to the length of time for which test conditions can be considered to be unchanged and stationary, or due to more practical reasons. For random processes these durations are nearly always much too short to enable an accurate determination of system behaviour. The 
measurements at Blyth were limited to 30 minute recordings of time series data. Using required analysis parameters (see section 6.2.2 and 6.2.3) this means that the processed spectra are the result of averaging over only 7 spectral estimates. The measured spectra are consequently subject to considerable statistical error (mostly random error, but bias errors can certainly not be excluded). The measured spectra are thus much more "irregular" and "peaky" than the much smoother, theoretically calculated spectra, which have been obtained by averaging over 100 spectral estimates.

These circumstances are always present and complicate comparisons by no small amount, the more so the more complicated the objectives become. The measured and calculated spectra for the mudline bending moment due to (nearly) wind only loading also suffered from the above, but showed very satisfactory agreement overall, see section 6.6.2 and Figure 6.15. For the more complex situation of combined wind and wave loading the differences in Figure 6.18 are bigger. However, taking the above considerations into account it may nonetheless be concluded that there is good general agreement:

- The measurements confirm the overall behaviour of the structure in bending for all four cases.

- The peak due to structural resonance around $0.47 \mathrm{~Hz}$ is clearly present in all spectra, but it would appear that the real Blyth support structure is either less strongly excited or more strongly damped than the model for cases 3, 4 and 5 . Lower unidirectional excitation on the real Blyth structure can be the result of sea states actually being multidirectional instead of unidirectional (see above). Higher damping for increasing wind speeds in cases 3,4 and 5 can be due to higher aerodynamic damping in reality, despite the opposite trend suggested in section 6.4 .

- The peak associated with 3P excitation in case 3 is evident in both the calculations and the measurements.

- Finally, the trend that quasi-static stress response increases with decreasing frequency below $0.4 \mathrm{~Hz}$ is confirmed as well. For cases 4 and 5 the measured stress response for frequencies in this range is lower, which can again be due to the fact that the sea states are actually multidirectional instead of unidirectional as in the model; unidirectional wave loading then clearly overestimates the bending stress response in the theoretical model. 


\subsection{Fatigue from response spectra}

\subsubsection{Introduction}

The preceding sections demonstrated the effective use of frequency domain analysis for the calculation of support structure stress response spectra. These spectra compare very well with spectra generated from time domain simulations. They are, however, only an intermediate step in the fatigue analysis. To complete this fatigue analysis, the following steps remain to be executed:

- $\quad$ count the stress variations $\left(S_{i}\right.$ and $\left.n_{i}\right)$

- compare stress variations to relevant design $S-N$ curve

- $\quad$ calculate the Miner sum fatigue damage $D_{\text {fat }}$

As presented in section 5.5, four methods of determining the stress variations will be investigated here. The general fatigue criteria and parameters are briefly described in section 6.8.2. The methods are detailed in 6.8.3 and 6.8.4. The final section gives the results for all methods and compares the outcome.

\subsubsection{Fatigue calculation criteria and parameters}

In the time domain, the rainflow counting algorithm can be used. In analysis reported in this thesis, the algorithm incorporated in the Bladed program has been used for all rainflow countings to make sure no deviations can occur due to counting method differences. The settings of the program require 2 inputs: minimum range to count, which is set to zero and number of bins, which is set to the maximum of 128 [78].

A relevant $S-N$ curve for a welded connection in the tubular was determined using the new issue (2004) of the GL design standard [64] after consulting their fatigue expert [79]. The $S-N$ curve is defined by:

$$
\log \left(N_{i}\right)=6.69897+m_{S N} \cdot Q
$$

with:

$$
Q=\log \left(\Delta \sigma_{R C} / S_{i}\right)-0.39794 / m_{S N 1}
$$

and:

\begin{tabular}{l|l|l}
$\Delta \sigma_{R C}$ & corrected reference fatigue strength at $N_{i}=5 \cdot 10^{6}$ & {$\left[\mathrm{~N} / \mathrm{m}^{2}\right]$} \\
$m_{S N}$ & inverse slope of $S-N$ curve & {$[-]$} \\
$m_{S N 1}$ & inverse slope of $S-N$ curve for $N_{i}<5 \cdot 10^{6}$, here $m_{S N 1}=3$ & {$[-]$} \\
$m_{S N 2}$ & inverse slope of $S-N$ curve for $N_{i}>5 \cdot 10^{6}$, here $m_{S N 2}=5$ & {$[-]$}
\end{tabular}

Table 6.7 shows the input correction factors for a submerged weld at the mudline as will be considered here. The weld is a "transverse loaded butt weld", ground flush to 
plate with $100 \%$ non-destructive testing, designated type 211 . The corrected reference strength is calculated with:

$$
\Delta \sigma_{R c}=f_{t, \sigma} \cdot f_{c, \sigma} \cdot f_{m, \sigma} \cdot f_{R, \sigma} \cdot f_{w, \sigma} \cdot f_{i, \sigma} \cdot f_{a, \sigma} \cdot \Delta \sigma_{R}
$$

Table 6.7 Correction factors for submerged weld according to GL [64] [79]

\begin{tabular}{l|l|l} 
factor & description & value \\
\hline \hline$f_{t, \sigma}$ & thickness effect & 0.803 \\
$f_{c, \sigma}$ & corrosion effect & 1.0 \\
$f_{m, \sigma}$ & material effect & 1.0 \\
$f_{R, \sigma}$ & effect of mean stress & 1.0 \\
$f_{w, \sigma}$ & effect of weld shape & 1.25 \\
$f_{i, \sigma}$ & influence of importance of structural & 1.0 \\
$f_{a, \sigma}$ & element & 1.0 \\
$\Delta \sigma_{R}$ & effect of misalignment & $100 \mathrm{~N} / \mathrm{mm}^{2}$ \\
& reference fatigue strength at $N_{i}=$ &
\end{tabular}

Figure 6.19 shows the $S-N$ curve for the detail that represents the structural element under consideration.
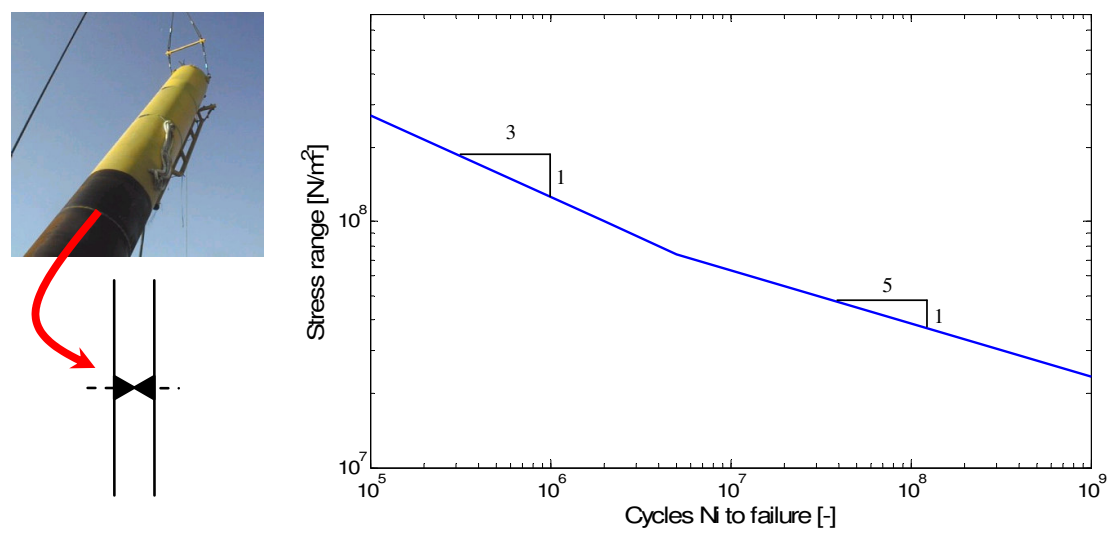

Figure 6.19 Upending of Blyth monopile with detail representing the mudline weld for $\sigma_{R}$ selection and related $S-N$ curve 


\subsubsection{Counting methods}

As discussed previously in sections 2.8 and 4.5, several methods of peak counting exist in the time domain with their equivalent method in the frequency domain. Table 6.8 gives an overview of these methods in both domains and a graphical representation.

Table 6.8 Methods of counting stress variations

\begin{tabular}{|c|c|c|}
\hline Time domain & Frequency domain & Graphic \\
\hline maximum peak counting & Rayleigh & $\eta \quad N^{N}$ \\
\hline all positive peak counting & Rice & \\
\hline rainflow counting & Dirlik & \\
\hline
\end{tabular}

All methods converge to a comparable outcome when applied to narrow-band signals. For broad-band signals, such as the response spectra under consideration in this chapter, the methods give increasingly dissimilar answers. This is demonstrated for the peak density distribution for case $5\left(V_{w}=18.7 \mathrm{~m} / \mathrm{s}, H_{s}=3.3 \mathrm{~m}, T_{z}=9.7 \mathrm{~s}\right)$ in Figure 6.20.

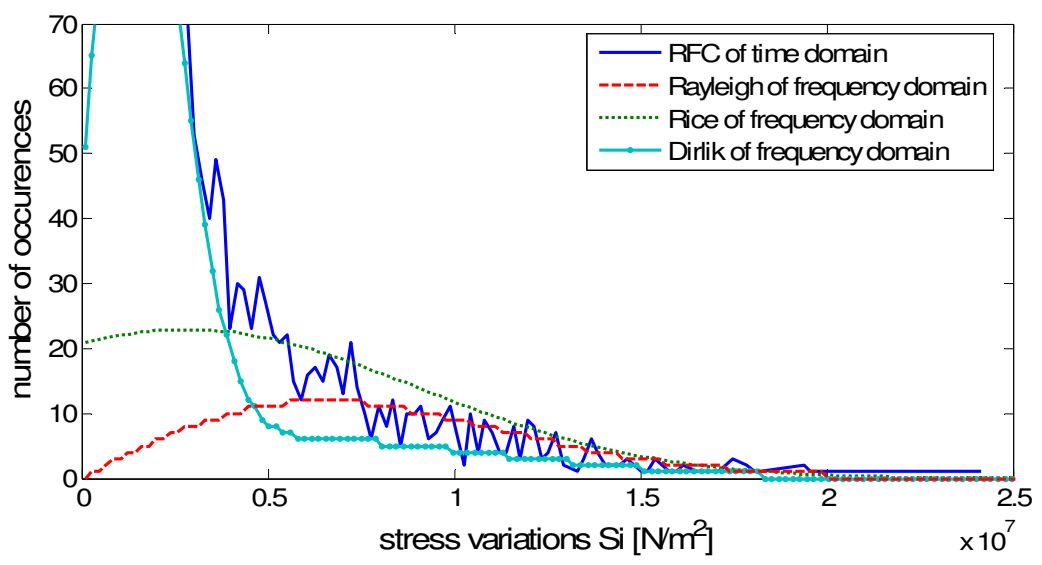

Figure 6.20 Comparison of rainflow counting outcome: number of occurrences $n$ of stress variations $S$ from direct time domain and IFFT frequency domain response 
As the methods (Rayleigh, Rice and Dirlik) incorporate an increasing number of spectral moments, the ability to incorporate the broad-band-effects of the stress response should improve. Dirlik is therefore expected to match the rainflow counted distribution closest. A comparison of counting methods is further addressed in 6.8.4 and section 7.4.

\subsubsection{Determining fatigue damage}

The different methods of determining the number of stress cycles in the frequency domain, and using that information for determining the fatigue damage with the selected $S-N$ curve, have been executed for all methods presented in section 5.5. Furthermore, the time domain output is rainflow counted, and output spectra are created from the time domain to also determine the fatigue damage through the Dirlik method. Figure 6.21 shows the flowchart for the post-processing operations carried out for the time domain and frequency domain responses.

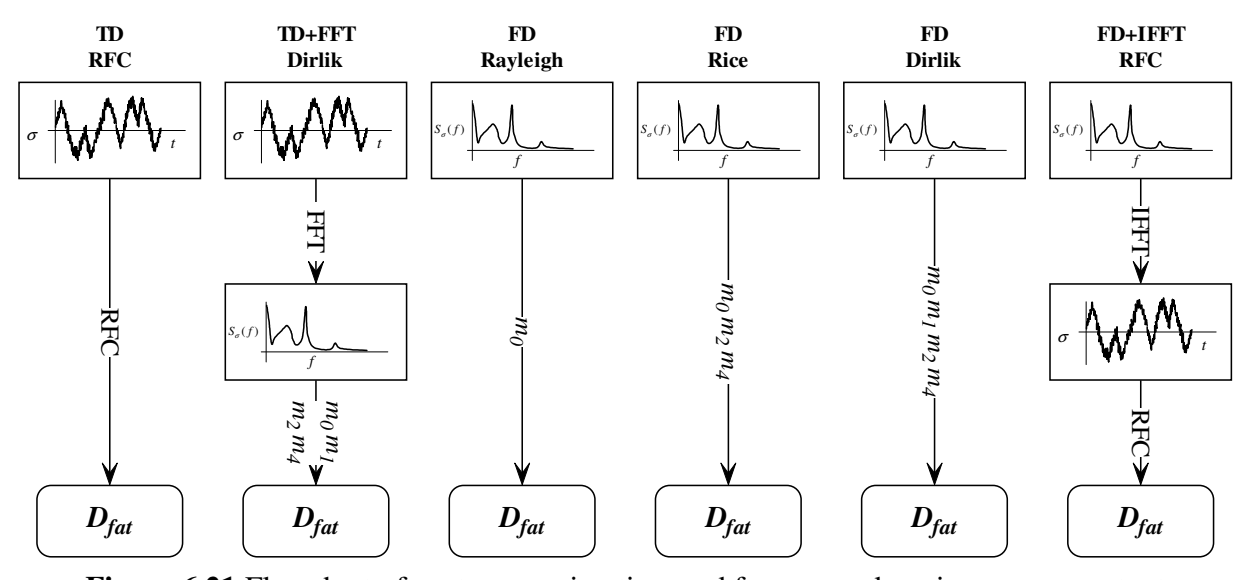

Figure 6.21 Flowchart of post-processing time and frequency domain response output to determine fatigue damage

Table 6.9 shows the outcome of fatigue damage $D_{\text {fat }}$ for all these cases for a full 20 year period. Table 6.10 shows the damages relative to the time domain-rainflow count. 
Table 6.9 Comparison of calculated fatigue damage for different methods for 5 reference states of the offshore wind turbines at Blyth. The tables represent $D_{f a t}$ for a service life of 20 years

\begin{tabular}{c|c|c|c|c|c|c} 
& $\begin{array}{c}\text { TD } \\
\text { RFC }\end{array}$ & $\begin{array}{c}\text { TD+FFT } \\
\text { Dirlik }\end{array}$ & $\begin{array}{c}\text { FD } \\
\text { Rayleigh }\end{array}$ & $\begin{array}{c}\text { FD } \\
\text { Rice }\end{array}$ & $\begin{array}{c}\text { FD } \\
\text { Dirlik }\end{array}$ & $\begin{array}{c}\text { FD+IFFT } \\
\text { RFC }\end{array}$ \\
\hline \hline case 1 & $1.86 \mathrm{E}-06$ & $1.70 \mathrm{E}-06$ & $1.12 \mathrm{E}-05$ & $1.72 \mathrm{E}-05$ & $1.26 \mathrm{E}-06$ & $1.68 \mathrm{E}-06$ \\
case 2 & $2.04 \mathrm{E}-05$ & $2.28 \mathrm{E}-05$ & $4.19 \mathrm{E}-05$ & $5.42 \mathrm{E}-05$ & $1.19 \mathrm{E}-05$ & $1.16 \mathrm{E}-05$ \\
case 3 & $2.04 \mathrm{E}-06$ & $1.86 \mathrm{E}-06$ & $1.17 \mathrm{E}-05$ & $1.77 \mathrm{E}-05$ & $1.52 \mathrm{E}-06$ & $1.60 \mathrm{E}-06$ \\
case 4 & $9.04 \mathrm{E}-05$ & $1.22 \mathrm{E}-04$ & $3.51 \mathrm{E}-04$ & $6.79 \mathrm{E}-04$ & $7.92 \mathrm{E}-05$ & $1.06 \mathrm{E}-04$ \\
case 5 & $1.29 \mathrm{E}-03$ & $1.34 \mathrm{E}-03$ & $2.26 \mathrm{E}-03$ & $2.55 \mathrm{E}-03$ & $1.10 \mathrm{E}-03$ & $1.08 \mathrm{E}-03$
\end{tabular}

Table 6.10 Comparison of the calculated fatigue damage of the offshore wind turbines at Blyth using different counting methods compared to the rainflow counting method (TD RFC) in \% for 5 reference states

\begin{tabular}{l|r|r|r|r|r|r} 
& \multicolumn{1}{|c|}{$\begin{array}{c}\text { TD } \\
\text { RFC }\end{array}$} & $\begin{array}{c}\text { TD+FFT } \\
\text { Dirlik }\end{array}$ & $\begin{array}{c}\text { FD } \\
\text { Rayleigh }\end{array}$ & $\begin{array}{c}\text { FD } \\
\text { Rice }\end{array}$ & $\begin{array}{c}\text { FD } \\
\text { Dirlik }\end{array}$ & $\begin{array}{c}\text { FD+IFFT } \\
\text { RFC }\end{array}$ \\
\hline \hline case 1 & $0 \%$ & $-9 \%$ & $500 \%$ & $825 \%$ & $-32 \%$ & $-10 \%$ \\
case 2 & $0 \%$ & $12 \%$ & $106 \%$ & $166 \%$ & $-42 \%$ & $-43 \%$ \\
case 3 & $0 \%$ & $-9 \%$ & $472 \%$ & $770 \%$ & $-26 \%$ & $-22 \%$ \\
case 4 & $0 \%$ & $35 \%$ & $289 \%$ & $651 \%$ & $-12 \%$ & $17 \%$ \\
case 5 & $0 \%$ & $4 \%$ & $75 \%$ & $98 \%$ & $-15 \%$ & $-16 \%$
\end{tabular}

The different processing steps for rainflow counting and Dirlik show remarkably good comparison. The reader is reminded that fatigue damage determination with $S$ - $N$ curves having slopes of 3 and 5 differ drastically for small changes in stress ranges. Especially for the spectra where wave induced stress becomes more important, cases 4 and 5 , the differences between frequency and time domain calculations become smaller.

The results show that Rayleigh and Rice deviate significantly from the rainflow counting and the Dirlik method. However, it should be noted that the calculated fatigue damages in table 6.9 are extremely low (from approximately $1.010^{-6}$ to $1.010^{-3}$ corresponding with fatigue lives of a thousand to a million years). Small absolute errors thus create large relative errors, as table 6.10 shows. The relevance of large relative differences between small numbers in practical applications can and should be questioned. This is further emphasized by the calculations in section 7.4, which relate to more realistic fatigue damage cases. These results will show that for such cases the different counting methods give comparable results. 


\subsection{Conclusions on frequency domain fatigue calculations}

In this chapter the frequency domain method was applied to the validated model of the Blyth offshore wind turbine. It is shown that effective uncoupling of the turbine and the support structure can be executed successfully, provided that the aerodynamic damping is taken into account as additional viscous damping in the support structure model. The calculated stress response spectra using the frequency domain method compare very well with the stress response spectra calculated via time domain simulations.

Through this uncoupling of the turbine and the support structure, wave induced response can be determined separately. The effects of wind and wave response can then be added by simple summation of the corresponding spectra. The results compare again very well with spectra from the time domain simulations.

Comparison of both time domain and frequency domain calculations to measured responses of the real turbine shows that the measurements incorporate numerous uncertainties, making model comparison to a single measurement campaign very difficult, even with a validated model.

To determine fatigue damage from the frequency domain response, several methods can be used. The support structures of the offshore wind turbines at Blyth are shown to be very insensitive to fatigue. The fatigue lives of points at the mudline run into the thousands of years. For these conditions the spread in calculated fatigue damage between different counting methods is considerable. Peak counting methods perform worst. The empirical Dirlik method uses most information from the spectra through 4 moments $\left(m_{0}, m_{1}, m_{2}, m_{4}\right)$ and shows the best comparison with the time domain method of rainflow counting as could probably be expected. As rainflow counting is the standard method of stress variation determination in the wind industry, the Dirlik method will most likely be favoured for frequency domain calculations.

This chapter proved that fatigue damage of offshore wind turbines can be determined effectively in the frequency domain by uncoupling the turbine from the support structure. In this chapter the comparison was made for single environmental states only. In the next chapter the method will be applied to the design of an offshore wind turbine in the Egmond aan Zee offshore wind farm (OWEZ) for the total fatigue damage calculation of all environmental states. 


\section{Frequency domain fatigue for OWEZ}

\subsection{Introduction}

The previous chapters proved that the proposed frequency domain method can be successfully applied to calculate fatigue damage of support structures for offshore wind turbines. The validated model of the offshore wind turbine at Blyth is the only one available for this demonstration, but is not the most suitable for parameter variation: the relatively high natural frequency of the structure prevents wave induced resonant behaviour, which minimizes the effect of fatigue damage on the support structure.

This chapter therefore describes the results of a study on a more representative structure to illustrate fatigue damage calculations in the frequency domain: the design for the OWEZ site. With a water depth of $20 \mathrm{~m}$ and a first natural frequency around $0.31 \mathrm{~Hz}$, the structure will show significant resonant behaviour, making fatigue damage a driving factor in the support structure design. Section 7.2 will first introduce the project details: site, turbine and environment. The analysis method currently favoured by most experts for time domain simulations is presented in section 7.3 together with the approaches to take the aerodynamic damping into account. In section 7.4 the fatigue damage checks for all operational load cases for the baseline design in both frequency and time domain are compared, including a more in-depth analysis of aerodynamic damping effects. With the frequency domain method working properly, parameter variations can be carried out quickly. In section 7.5 this is illustrated by subjecting the baseline design to variations in scour depth and calculating its effects on fatigue life. The conclusions on frequency domain fatigue damage calculation are summarized in the final section 7.6.

\subsection{Description of turbine, support structure and environment}

\section{The turbine}

General characteristics of the OWEZ project were introduced in chapters 1 and 3. The OWEZ project has been in the pipeline for a very long period before scheduled installation in 2006. During this period, a complete and certified design has been made for the NEG-Micon NM92 turbine in 2004. Due to the merger of NEG-Micon and Vestas during 2004, the choice of turbine changed to the Vestas V90. The calculations in this chapter are based on the final design for the abandoned NM92 turbine. Through co-operation with NEG-Micon the turbine model in Bladed was validated with measurements and design software output. 


\section{The support structure}

For the support structure, several options were detailed: with and without scour protection and with different options for the connection of the transition piece to the foundation pile. Only one design was made for all 36 locations in the offshore wind farm, based on a maximum water depth in the installation area of $20 \mathrm{~m}$. Figure 7.1 shows this baseline design including a $8.5 \mathrm{~m}$ scour hole without scour protection; this is a scour depth of 1.8 times the pile diameter. For all calculations in this chapter, except the scour depth variation calculations in section 7.5 , the scour depth of this baseline design is being used.

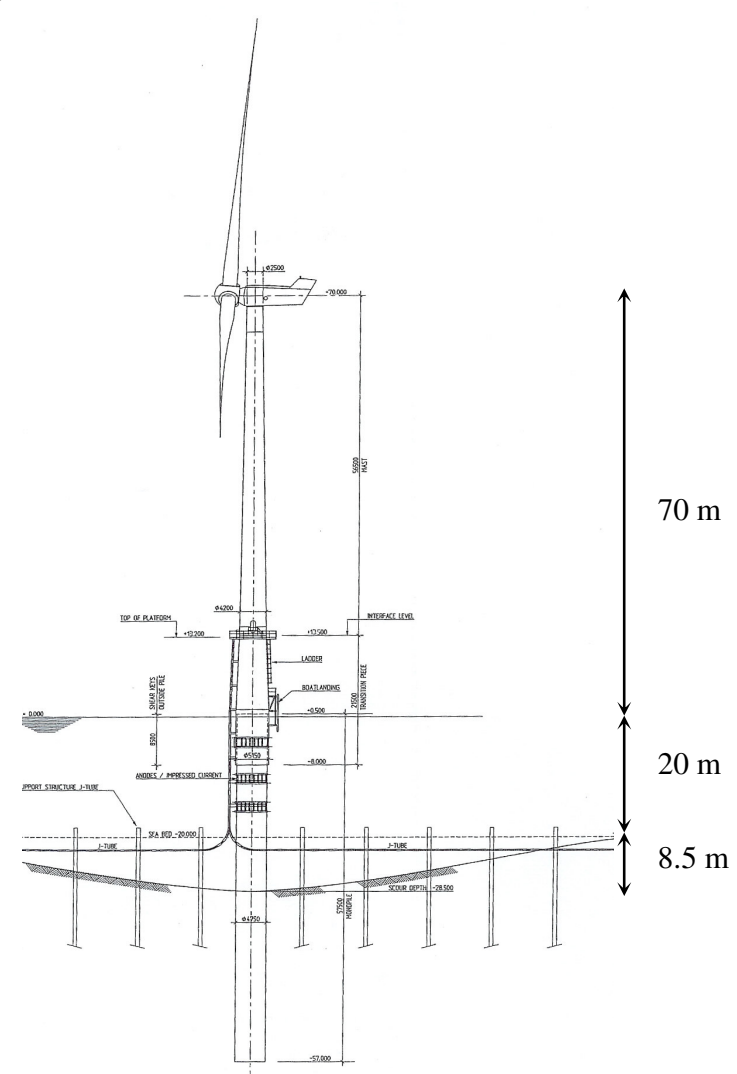

Figure 7.1 Drawing of OWEZ baseline design for the NM92 turbine

\section{The environment}

It is standard practice to use databases of wind, wave and current to find the simultaneous occurrence of all parameters. As current produces a constant load on the 
structure during the environmental states defined for fatigue analysis, it is not taken into account for the analysis of the steel support structures considered here: constant stress is not supposed to add to fatigue damage for steel structures (it does for composites and concrete, for instance). Directionality is normally reduced to bins of $30^{\circ}$ with incorporation of symmetry effects: due north and due south excitations by wind and waves induce fatigue damage at the same locations in the structure. For preliminary design no directional effects are taken into account: all wind and waves are modelled as unidirectional and coming from one direction.

The mean wind speed, significant wave height and zero-crossing wave period are usually gathered in a three-dimensional scatter diagram. To create such a diagram, two methods exist:

- $\quad$ binning wave parameters to an $H_{s}-T_{z}$ wave scatter diagram and then adding the percentage of occurrence of different mean wind speeds per scatter diagram bin;

- $\quad$ making a wave scatter diagram per mean wind speed range, in steps of $2 \mathrm{~m} / \mathrm{s}$. The first method is clearly based on the offshore way of looking at the data, while the second is assessing the data from the turbine point of view. With respect to a standardizing the analysis method and to suit the proposed frequency domain method, the wind-based method is clearly preferable. Only a limited number of wind speed cases need to be addressed in time domain simulations with a rigid support structure, with each simulation serving a complete wave scatter diagram per wind speed class. Recent developments [24] have incorporated this type of 3D scatter diagram option in environmental databases for downloading of all global offshore sites.

In the time domain, further reduction of environmental states can be achieved by binning nearly similar states to a mean state. This will reduce the number of load cases from a typical 100 to approximately 15 . The reduction method works well with respect to reducing calculation effort and maintaining accuracy [3][4]. Clearly, the frequency domain method is superior in this respect as it does not require any reduction in the preliminary phase to enable even faster calculations.

For the environmental data, the NESS-NEXT data is used in this thesis. The data has been grouped into $2 \mathrm{~m} / \mathrm{s}$ wind speed bins for $0.5 \mathrm{~m} H_{s}$ bin sizes and $1 \mathrm{~s} T_{z}$ bin sizes. This results in 112 environmental states for which the turbine is operating. The non-operating states are left out of the present analysis as they give comparable results for time and frequency domain methods; this should of course not be done in a detailed design effort. Figure 7.2 shows part of the scatter diagram for $V_{w}=10 \mathrm{~m} / \mathrm{s}$. The full 3D scatter diagram is shown in appendix II, the summary of environmental parameters per state are shown in appendix III. 


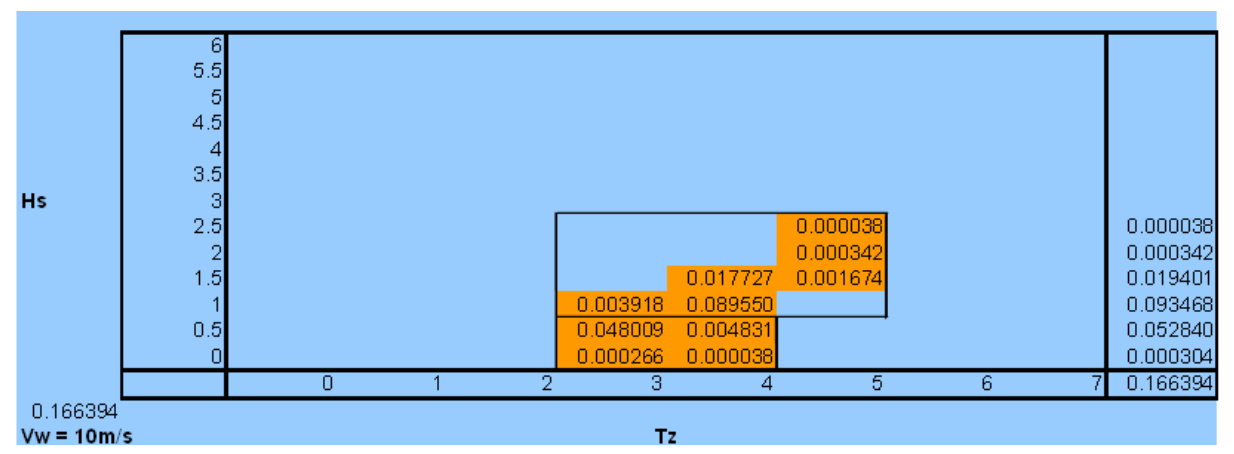

Figure 7.2 Part of 3D scatter diagram for $V_{w}=10 \mathrm{~m} / \mathrm{s}$

For quick time domain checks, the 112 states are further binned to 22 representative states as shown in table 7.1 [84].

Table 7.1 22 binned environmental states

\begin{tabular}{c|c|c|c|c} 
State & $V_{w}$ & $T_{z}$ & $H_{s}$ & $P($ state $)$ \\
\hline \hline 1 & 4 & 3 & 0.5 & 0.039525 \\
2 & 4 & 4 & 0.5 & 0.032107 \\
3 & 6 & 3 & 0.5 & 0.111652 \\
4 & 6 & 4 & 0.5 & 0.072241 \\
5 & 8 & 3 & 0.5 & 0.114467 \\
6 & 8 & 4 & 1.0 & 0.086849 \\
7 & 10 & 3 & 0.5 & 0.053144 \\
8 & 10 & 4 & 1.0 & 0.113250 \\
9 & 12 & 4 & 1.0 & 0.058584 \\
10 & 12 & 4 & 1.5 & 0.059992 \\
11 & 14 & 4 & 1.5 & 0.044775 \\
12 & 14 & 5 & 2.0 & 0.032640 \\
13 & 16 & 4 & 2.0 & 0.017880 \\
14 & 16 & 5 & 2.5 & 0.031042 \\
15 & 18 & 5 & 2.5 & 0.017385 \\
16 & 18 & 5 & 3.0 & 0.008027 \\
17 & 20 & 5 & 2.5 & 0.004337 \\
18 & 20 & 5 & 3.0 & 0.011412 \\
19 & 22 & 5 & 3.0 & 0.003956 \\
20 & 22 & 6 & 4.0 & 0.002929 \\
21 & 24 & 5 & 3.5 & 0.001522 \\
22 & 24 & 6 & 4.0 & 0.000989
\end{tabular}




\subsection{Set-up of fatigue checks}

\subsubsection{Simulation settings}

As presented in section 5.6, the expert advice on the time step was to use $0.1 \mathrm{~s}$. In section 6.2 .2 a time step of $0.25 \mathrm{~s}$ was found to be sufficient, but the aliasing effect described in section 6.2.3 was also found for this model with this time step size. The aliasing is prevented when the time step size was reduced to $0.125 \mathrm{~s}$.

Following the expert opinions (section 5.6), a single simulation of $3600 \mathrm{~s}$ will be carried out per environmental state, with a non-recorded initiation time of $120 \mathrm{~s}$, which was found to be sufficient to let initiation effects die out. When spectra are created from these time series for comparison with the frequency domain using a subrecord length of 4096 points (512 s), the resulting spectrum will be an average of only 14 subrecords.

\subsubsection{Aerodynamic damping}

The aerodynamic damping was estimated using the Garrad method and the non-linear simulation method. The results are shown in Figure 7.3, together with the engineering estimate of $4 \%$.

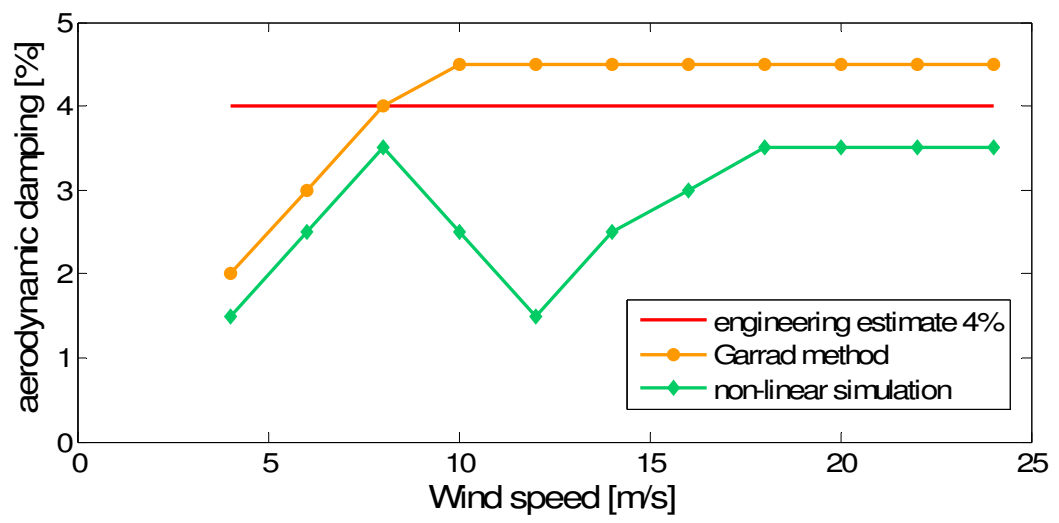

Figure 7.3 Aerodynamic damping for NM92 from the Garrad method (red), the non-linear simulation (purple) and the engineering estimate of $4 \%$ (blue)

\subsubsection{Preliminary comparison of response spectra}

Before calculating the fatigue damage in both frequency and time domain, the response spectra for the two methods are compared for a few cases. As for the offshore wind turbine at Blyth in the previous chapters, the response spectra for different load 
cases match very well, as shown by way of example in Figure 7.4 for case 42 with $V_{w}=$ $10 \mathrm{~m} / \mathrm{s}, H_{s}=1 \mathrm{~m}$ and $T_{z}=4 \mathrm{~s}$. Comparison of all 112 states is shown in appendix IV.

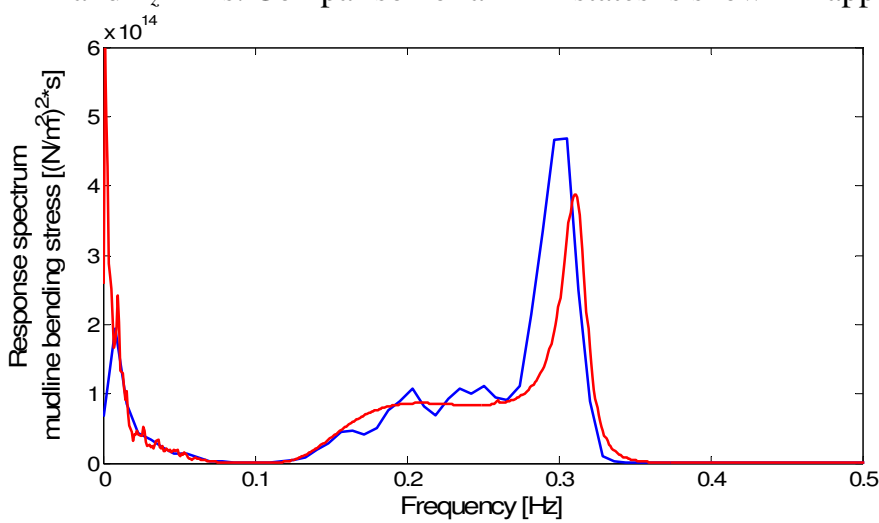

Figure 7.4 Comparison for time domain (blue) and frequency domain (red) response spectra for: $V_{w}=10 \mathrm{~m} / \mathrm{s}, H_{s}=1 \mathrm{~m}$ and $T_{z}=4 \mathrm{~s}$

The response spectra clearly show the much more prominent resonance peak at 0.31 $\mathrm{Hz}$. Due to the lower natural frequency than the Blyth offshore wind turbine, a larger portion of wave spectral energy is projected across the natural frequency, resulting in more pronounced resonant behaviour. Figure 7.5 shows the effect of aerodynamic damping: when incorporated the resonance peak reduces significantly.

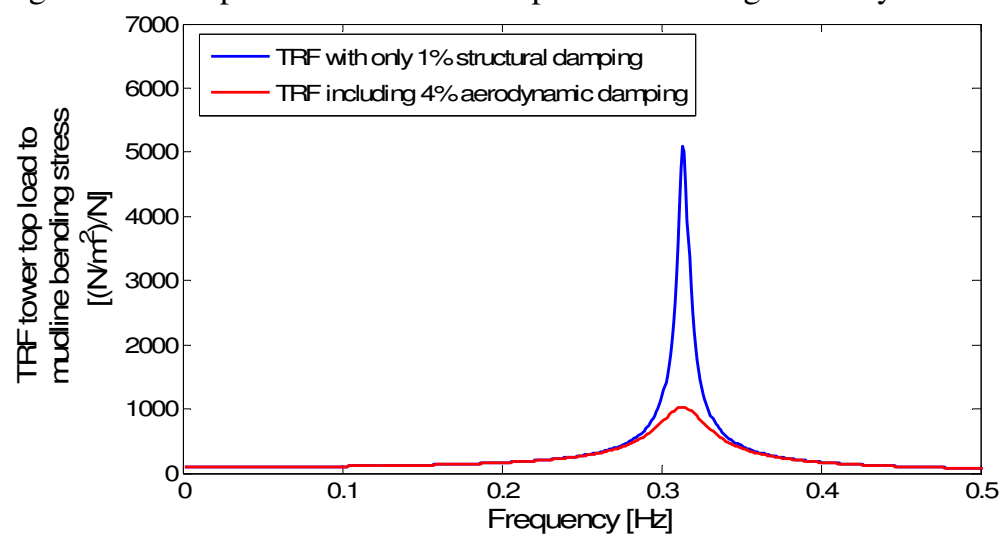

Figure 7.5 Effect of aerodynamic damping on the transfer functions for bending stress at the mudline per unit tower top load 


\subsection{Fatigue calculations for the baseline design}

\subsubsection{Comparison of full and reduced time domain calculations}

A first method to speed-up fatigue assessment in the time domain is to reduce the number of environmental states. As discussed in section 7.2, the full 112 states were reduced to 22 representative states. As the effects of the random seeds on a reduced number of states may become more significant, it could be advisable to make a number of random realisations of these states and take their mean. The results of the fatigue checks are shown in table 7.2.

Table 7.2 Comparison of lifetime fatigue damage for time domain simulation of the full 112 states and of 5 runs each of the reduced number of 22 states and their mean

\begin{tabular}{c|c|c|c|c|c|c} 
Time domain & \multicolumn{6}{|c}{ Time domain 22 states, 5 runs } \\
\cline { 2 - 7 } 112 states & 1 & 2 & 3 & 4 & 5 & $\begin{array}{c}\text { mean of 5 } \\
\text { runs }\end{array}$ \\
\hline \hline 0.56 & 0.58 & 0.60 & 0.63 & 0.62 & 0.62 & 0.61
\end{tabular}

It can be seen that the reduction of the number of states from 112 to 22 has resulted in a conservative representation, always resulting in slightly higher fatigue damage for each random simulation of the 22 states. The mean of the 5 runs is a good conservative estimate compared to the full 112 states simulation.

\subsubsection{Comparison of time and frequency domain calculations for 112 states}

The next step is to compare the full 112 states time domain fatigue calculations with the results of the frequency domain approach. For this case the engineering estimate of constant aerodynamic damping of $4 \%$ is used for all wind speed bins. Furthermore, two frequency domain representations of wave response are calculated: with and without diffraction correction. For the Blyth offshore wind turbine, with its natural frequency at $0.5 \mathrm{~Hz}$, diffraction significantly reduced the height of the resonance peak in the transfer function for wave loads as shown in Figure 6.16. As Bladed uses a spectral cut-off of the input wave spectrum, diffraction could have been implicitly incorporated in the time domain simulations for Blyth and possibly also for OWEZ. The diffraction correction is a standard option in the SESAM design program and by switching it on and off, its impact on the design can be analysed. Table 7.3 shows the results for the three fatigue checks. The frequency domain fatigue damage was calculated with the Dirlik method. 
Table 7.3 Comparison of fatigue damage for time domain and frequency domain without and with diffraction correction

\begin{tabular}{c|c|c}
$\begin{array}{c}\text { TD 112 states } \\
\text { Bladed cut-off }\end{array}$ & $\begin{array}{c}\text { FD 112 states } \\
\text { no diffraction }\end{array}$ & $\begin{array}{c}\text { FD 112 states } \\
\text { with diffraction }\end{array}$ \\
\hline \hline 0.56 & 0.50 & 0.16
\end{tabular}

From the comparison of both frequency domain calculations it is apparent that also for the OWEZ offshore wind turbine the diffraction reduces wave induced stresses significantly. This effect is included in the Bladed program as a spectral cut-off. For this particular structure and water depth the cut-off occured at a frequency above the natural frequency, therefore not affecting the outcome. The impact of the diffraction correction can also be visualised in the MacCamy-Fuchs graph presented earlier in Figure 2.15. Taking the deep water relation for wave length to wave period, which is true for the fatigue inducing waves at $20 \mathrm{~m}$ water depth:

$$
\lambda_{\text {wave }}=1.56 T^{2}
$$

it can be calculated that for the support structure with $D=4.75 \mathrm{~m}$ at the natural period of approximately $3 \mathrm{~s}$, the ratio $D / \lambda_{\text {wave }}=0.34$, giving a reduced inertia coefficient of $C_{m}=1.3$ at the resonant frequency compared to $C_{m}=2.0$ when no correction is applied. The corrected value is indicated in Figure 7.6.

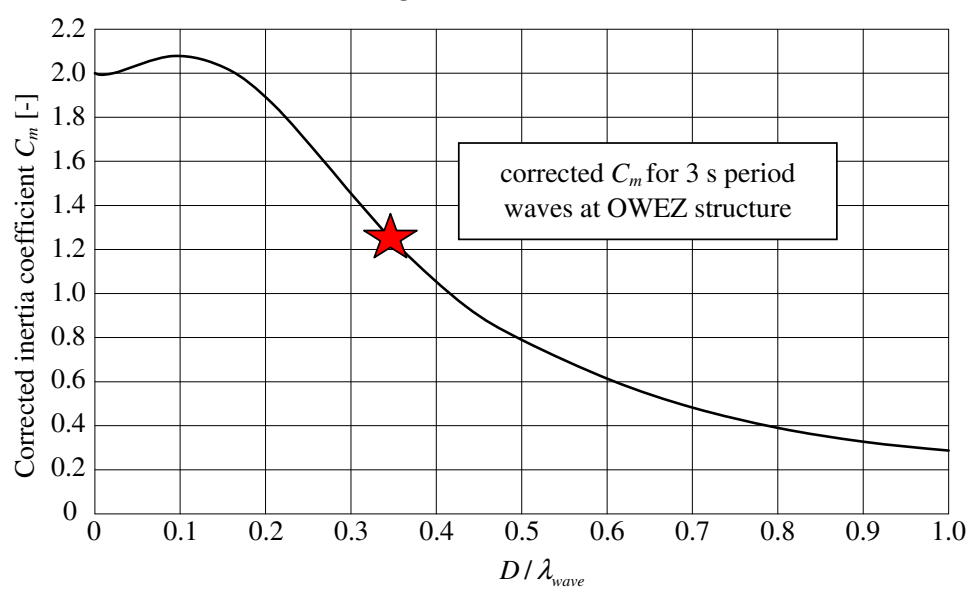

Figure 7.6 MacCamy-Fuchs diffraction correction of the inertia coefficient $C_{m}$ and the required correction for the OWEZ structure at the natural frequency (star) 
The time domain calculations in Bladed do not incorporate any diffraction correction and should hence only be used for comparison with non-corrected frequency domain fatigue damage. Table 7.3 clearly shows that the non-corrected frequency domain fatigue damage compares very well with the time domain calculated fatigue damage. Although the frequency domain value is also lower, a deviation of roughly $10 \%$ on lifetime fatigue is by all means acceptable for a preliminary design calculation based on the very crude estimate of the aerodynamic damping.

A more detailed study of the damage per wind speed bin reveals that the differences between time and frequency domain fatigue damage outcome show a trend over the wind speed bins: for lower wind speed bins, the frequency domain method calculates lower fatigue damage, for higher wind speed bins, the fatigue damage in the frequency domain is higher than in the time domain. This effect can be explained by the aerodynamic damping being dependent on the wind speed: for low wind speeds, the turbine induces less damping, making the estimate of $4 \%$ too high resulting in lower fatigue damage in the frequency domain. For higher wind speeds, the $4 \%$ damping is too low, resulting in more fatigue damage in the frequency domain calculations, as can be seen in Figure 7.7.

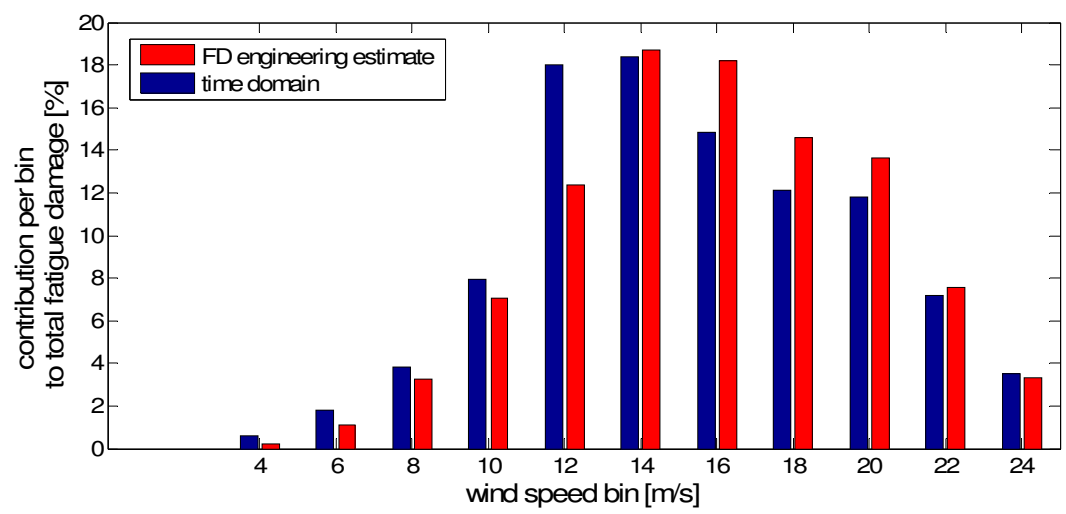

Figure 7.7 Comparison of fatigue damage per wind speed bin for time and frequency domain calculations for $4 \%$ aerodynamic damping

In the frequency domain, several probability distributions can be used to find the number of stress range variations: Rayleigh, Rice and Dirlik. The results of these methods can also be compared for the frequency domain check with the engineering estimate of $4 \%$ aerodynamic damping and no diffraction correction. Table 7.4 shows these results. As found in section 6.8.4, Rice and Rayleigh give higher fatigue damage values. However, for the OWEZ case in which the calculated fatigue damage is much more a design driver and has therefore more realistic and meaningful values than for 
Blyth, the fatigue damages calculated with the various distributions compare much better, both mutually and with the rainflow counting method of the time domain simulations. The Rayleigh and Rice calculations are slightly conservative compared to rainflow counting, the Dirlik calculation is slightly optimistic.

Table 7.4 Comparison of lifetime fatigue damage for time domain and frequency domain using Rayleigh, Rice and Dirlik distributions for $4 \%$ aerodynamic damping

\begin{tabular}{c|c|c|c}
$\begin{array}{c}\text { TD 112 states } \\
\text { RFC }\end{array}$ & $\begin{array}{c}\text { FD 112 states } \\
\text { Rayleigh }\end{array}$ & $\begin{array}{c}\text { FD 112 states } \\
\text { Rice }\end{array}$ & $\begin{array}{c}\text { FD 112 states } \\
\text { Dirlik }\end{array}$ \\
\hline \hline 0.56 & 0.58 & 0.58 & 0.50
\end{tabular}

\subsubsection{Impact of different estimates of aerodynamic damping on the lifetime fatigue damage}

As shown in Figure 7.7, the single aerodynamic damping value of $4 \%$ for all wind speed classes results in differences in the time domain calculations for low and high wind speeds. As presented in section 5.3.2 several calculation methods exist for the determination of the aerodynamic damping. To facilitate the calculation of these wind speed class dependent damping values, transfer functions were created for total (structural + aerodynamic) damping of $1 \%$ to $10 \%$ in steps of $0.5 \%$.

The results of the different aerodynamic damping input is shown in table 7.5 together with damage due to the single aerodynamic damping value of $4 \%$ presented in the previous section and the time domain fatigue damage. All results exclude the influence of diffraction correction.

Table 7.5 Fatigue damage results (Dirlik) for time domain and three different aerodynamic damping realisations per wind speed bin

\begin{tabular}{c|c|c|c}
$\begin{array}{c}\text { TD 112 states } \\
1 \text { hour } \\
\text { simulations }\end{array}$ & $\begin{array}{c}\text { FD 112 states } \\
\text { engineering } \\
\text { estimate }(4 \%)\end{array}$ & $\begin{array}{c}\text { FD 112 states } \\
\text { Garrad method }\end{array}$ & $\begin{array}{c}\text { FD 112 states } \\
\text { non-linear } \\
\text { simulation }\end{array}$ \\
\hline \hline 0.56 & 0.50 & 0.45 & 0.74
\end{tabular}

For the accumulated fatigue damage, the figures are all of the same order of magnitude. To study the details of the different aerodynamic damping calculation methods, the differences between the cases per wind speed bin can again be plotted against the reference time domain case as shown in Figure 7.8. 

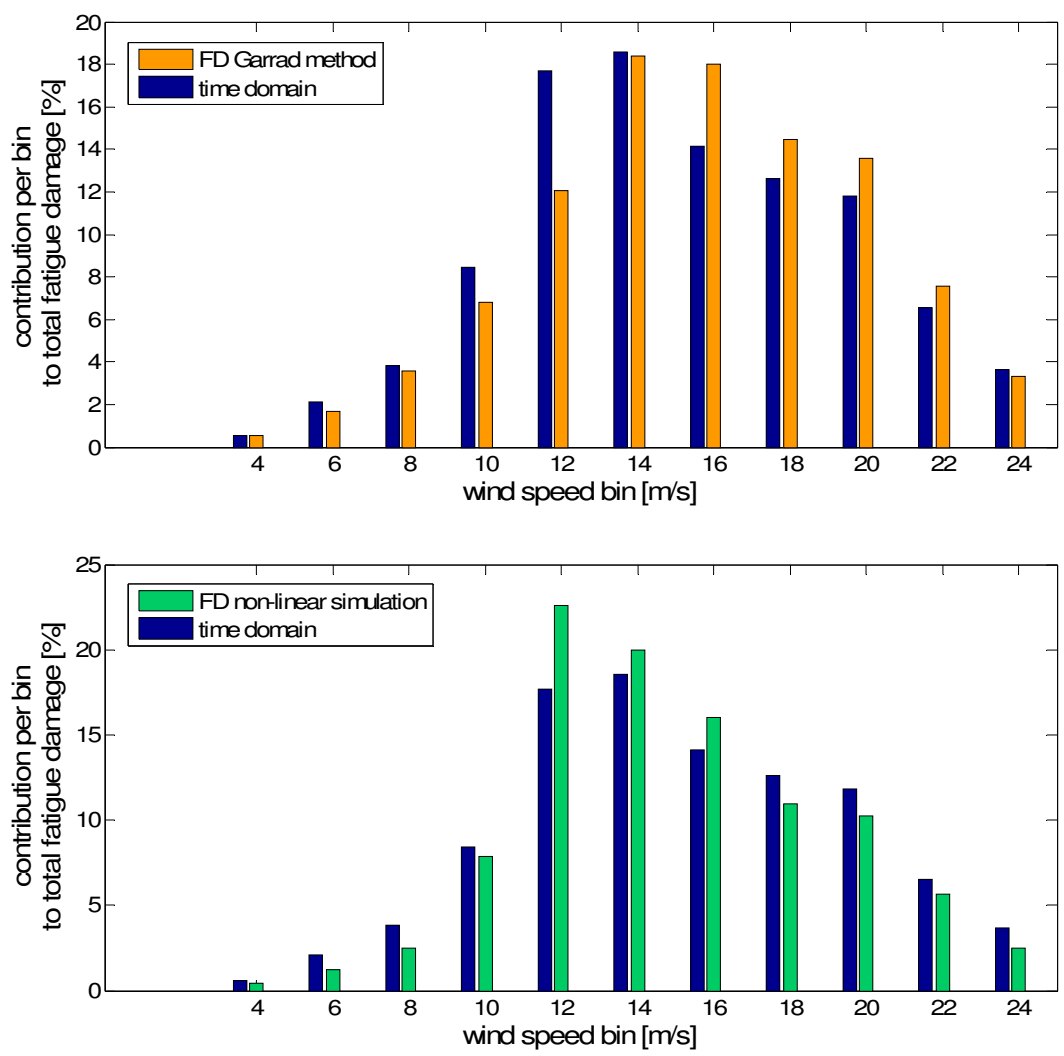

Figure 7.8 Comparison of fatigue damage per wind speed bin for time and frequency domain calculations with the aerodynamic damping from the Garrad method (above) and the non-linear simulation method (below)

Figure 7.8 shows that the fatigue damages for the different damping estimates remain slightly different from the time domain fatigue damage. The main findings are that damage for low wind speed classes is consistently lower and that for high wind speed classes the damage is consistently higher than the time domain results.

Although the Garrad method incorporates the effects of the variable speed rotor, reducing aerodynamic damping for lower wind speeds, the effect on the fatigue damage is only small and is counteracted by a higher value of aerodynamic damping (4.5\%) for above rated wind speeds, further reducing fatigue damage compared to the $4 \%$ engineering estimate case. The total fatigue damage is therefore somewhat lower than for the engineering estimate case.

The non-linear simulation method resulted in a significantly lower aerodynamic damping value, especially for the above rated wind speeds (Figure 7.3). From the 
comparison per wind speed bin in Figure 7.8 it is apparent that for these bins the frequency domain calculated fatigue damage and the time domain calculated fatigue damage match better. The dent in the aerodynamic damping at the 10,12 and $14 \mathrm{~m} / \mathrm{s}$ wind speed bins compares well with the trend in the time domain calculated fatigue damage, although it overshoots slightly.

From an engineering point of view, the single engineering estimate of $4 \%$ damping for all wind speed bins gives a very good first stab at assessing fatigue damage. When more turbine details are available, the non-linear simulation method gives a better figure for the aerodynamic damping per bin.

\subsubsection{Manual fit of aerodynamic damping}

As the fatigue damage calculation tool in the frequency domain method can easily cope with parameter variation, a purely academic fit of damping can be made to make time and frequency domain fatigue damage agree more. The aerodynamic damping per wind speed bin was varied until the mean fatigue damage between time and frequency domain differed by less than $10 \%$ per wind speed bin. This fitting method assumes that the difference between time and frequency domain fatigue damage calculation per environmental state can be minimised by "tuning" the aerodynamic damping. It is used here as a test case to get more feeling for the aerodynamic damping variation per wind speed bin. The outcome per wind speed class is shown in Figure 7.9. In appendix IV the stress response spectra from the time and frequency domain method are compared.

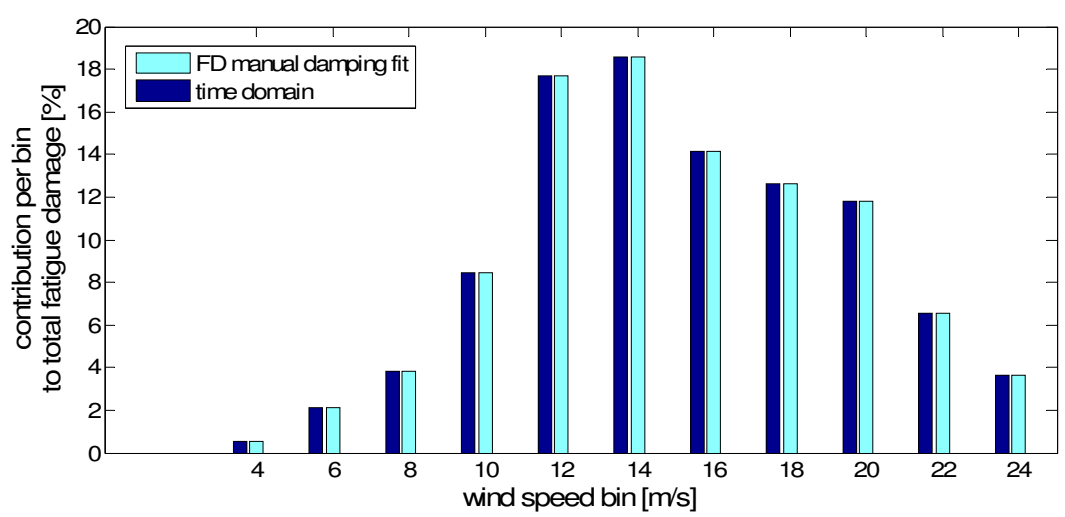

Figure 7.9 Fitted aerodynamic damping per wind speed class to make the frequency domain calculated fatigue (Dirlik) and time domain (RFC) calculated fatigue the same

The total fatigue damage is shown in table 7.6. As before, the results exclude the diffraction correction. The Dirlik result, for which the damping was fitted, now indeed 
gives the same fatigue damage as the rainflow counting. The Rayleigh and Rice distributions, for the same damping as found for the Dirlik fitting, give some $20 \%$ higher damage results.

Table 7.6 Comparison between time domain fatigue damage and frequency domain fatigue damage for manually fitted aerodynamic damping

\begin{tabular}{c|c|c|c}
$\begin{array}{c}\text { TD 112 states } \\
\text { RFC }\end{array}$ & \multicolumn{3}{|c}{ FD 112 states with manually fitted damping } \\
Dirlik & Rayleigh & Rice \\
\hline \hline 0.56 & 0.57 & 0.67 & 0.67
\end{tabular}

The damping found through this manual fitting method is discussed in [80] together with the other damping values determined previously. The fitting must be handled with care as it is only a variation of end results based on a desired outcome of end results. And it is not only the aerodynamic damping that is responsible for differences between time and frequency domain results. The four aerodynamic damping methods are depicted in Figure 7.10

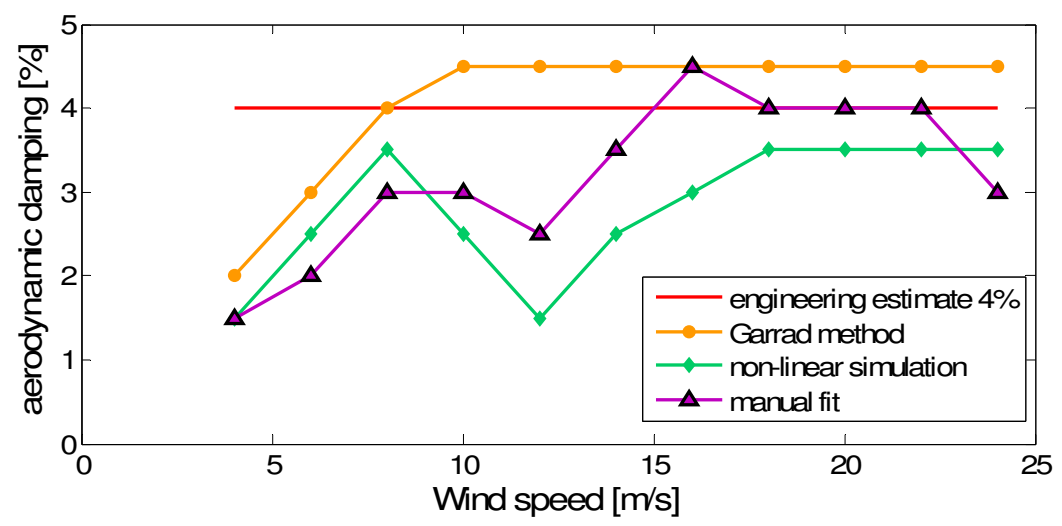

Figure 7.10 Aerodynamic damping for NM92, engineering estimate (blue), Garrad method (red, bullets), non-linear simulation (purple, diamonds), manual fit (green, triangles)

The manually fitted aerodynamic damping clearly mimics the trend found with the non-linear simulation method for aerodynamic damping. The method could be used to further optimise the non-linear simulation method and the understanding of the phenomena influencing the aerodynamic damping. Should the manual fit be correct, then the following observations on aerodynamic damping could be made:

- clear impact of variable rotor speed at partial load wind speeds

- decreased damping as the turbine reaches the rated wind speed 
- $\quad$ small overshoot at rated wind speed where rotation speed control and blade pitch control interchange power output control (could be a modelling feature)

- decrease in aerodynamic damping near cut-out (could also be a modelling feature).

\subsubsection{Conclusions on aerodynamic damping}

The main conclusion to be drawn from the results of the different aerodynamic damping calculation methods is that this subject requires more research to come to a fully verified calculation method. The aerodynamic damping has significant effect on the fatigue life of the support structure, which is explicitly demonstrated in the frequency domain but only implicitly included in the time domain calculation. The impact of control routines on the aerodynamic damping are apparent in the time domain simulation software and these should be checked for physical correctness by measurements from real offshore wind turbines.

In the meantime, the engineering estimate of $4 \%$ aerodynamic damping seems to be giving the most usable estimate of aerodynamic damping for preliminary design purposes for the class of turbines assessed in this thesis: 2-3 MW with a first natural frequency around $0.3 \mathrm{~Hz}$ or higher. Although variations are noted in a detailed study of the results for the 112 cases considered, the final summation to obtain the total fatigue damage does not seem to be severely affected as differences tend to average out.

When measurements from more turbines become available, the non-linear simulation method may give much more refined establishment of the aerodynamic damping per wind speed bin. This method could be used by turbine manufacturers to provide an aerodynamic damping table per wind speed bin for their turbine models, just as a typical power curve or the dimensions and weights of the individual components.

Although uncertainties regarding the absolute value of aerodynamic damping remain, the different methods give ample insight to effectively apply the frequency domain fatigue calculation method.

\subsection{The effect of scour on the lifetime fatigue damage}

\subsubsection{Using frequency domain fatigue calculations for sensitivity analyses}

As offshore wind energy is gradually becoming a mature technology, various aspects of placing turbines offshore still give rise to questions resulting in uncertainty, to overdimensioning and to increase of cost. One of these major uncertainties is the effect of scour on the dynamics of the offshore wind turbine and on its fatigue life. To discard these uncertainties, one could opt for scour protection, fixing the seabed level and 
preventing scour. But this option is costly and scour protection requires maintenance during the service life of the offshore wind farm.

The frequency domain fatigue damage calculation method offers the option to quickly assess the impact of different scour depths on the dynamics and fatigue life of the support structure. In this way, although the true extent of the scour depth may be uncertain, its effects can be checked. This supports more educated design decisions, which could eventually reduce offshore wind farm costs.

\subsubsection{General description of scour around monopile structures}

As described briefly in section 3.3 scour is the process where the current accelerates around the support structure and due to this acceleration picks up and transports soil particles away from the support structure. Figure 7.11 shows the scour process and an example of scour around a test pile.

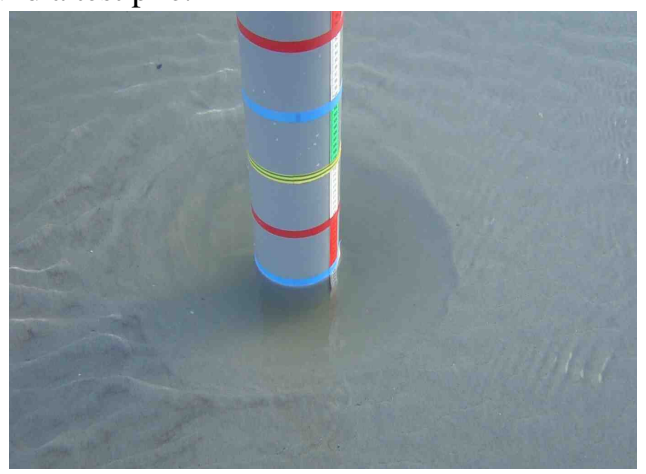

Figure 7.11 Scour around a test pile

Scour affects the design of offshore wind turbines in three ways:

- lowering of the seabed around the structure reduces the lateral bearing resistance that the foundation pile can mobilize, which may mean that the pile needs to be driven deeper into the seabed,

- the lowering of the seabed effectively makes the entire support structure longer, lowering the natural frequency, which can have implications for fatigue damage,

- a large scour hole will leave the J-tube free-spanning, which eventually may damage the cable if this effect is not taken into account properly.

To prevent scour, a layer of crushed rock can be dumped around the support structure, thus increasing the particle size and weight to prevent it from being picked up and transported. Although rock dumping is effective, the scour protection requires inspection and maintenance throughout the lifetime of the offshore wind farm. On the 
other hand, when the extent of the scour can be predicted, its effects can be taken into account in the design, reducing cost. A literature survey was carried out in [63], which pointed to the conclusion that current design standards may over-estimate the scour depth, by recommending a depth of 1.5 to 2.7 times the pile diameter $D$. The few measurements available from large diameter monopiles reveal that a scour depth of less than $1 D$ has been found.

\subsubsection{Scour depth variations}

To assess the impact of scour on the fatigue design, the scour depth is varied from no scour to a maximum of $2.5 \mathrm{D}$. To model the foundation stiffness, the soil layers are represented by $p-y$ curves. For every scour depth a stiffness matrix is created which is fed into SESAM to derive the different transfer functions. As this method of foundation simulation was not used in the previous sections, for this scour check a small deviation of the natural frequency occurred when compared to the model of the structure in the previous sections.

The dimensions of the structure are not altered throughout the parameter variation, but reduced fatigue damage due to less scour could eventually lead to a structure requiring less steel and therefore being cheaper. Figure 7.12 shows the decrease of the natural frequency as the scour depth increases.

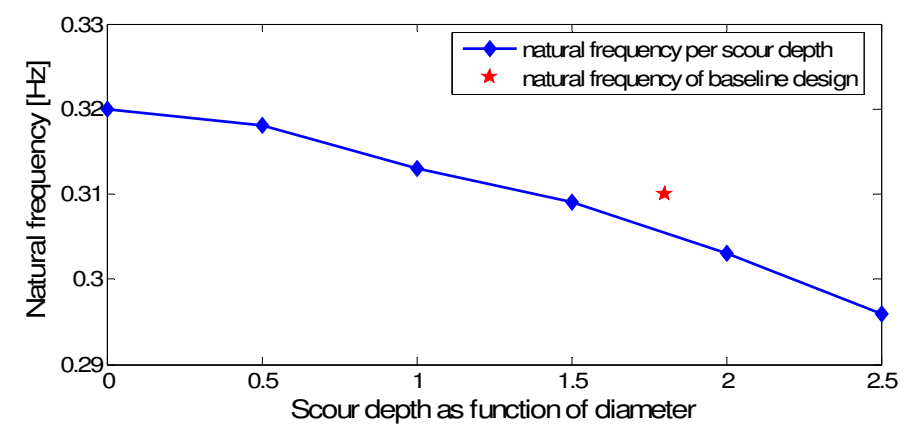

Figure 7.12 Natural frequency change due to increasing scour depth (blue) and natural frequency of baseline design with $1.8 D$ scour depth (red)

For these checks the engineering estimate of $4 \%$ aerodynamic damping has been used and diffraction correction has been excluded. The results of the fatigue checks are shown in Figure 7.13. 


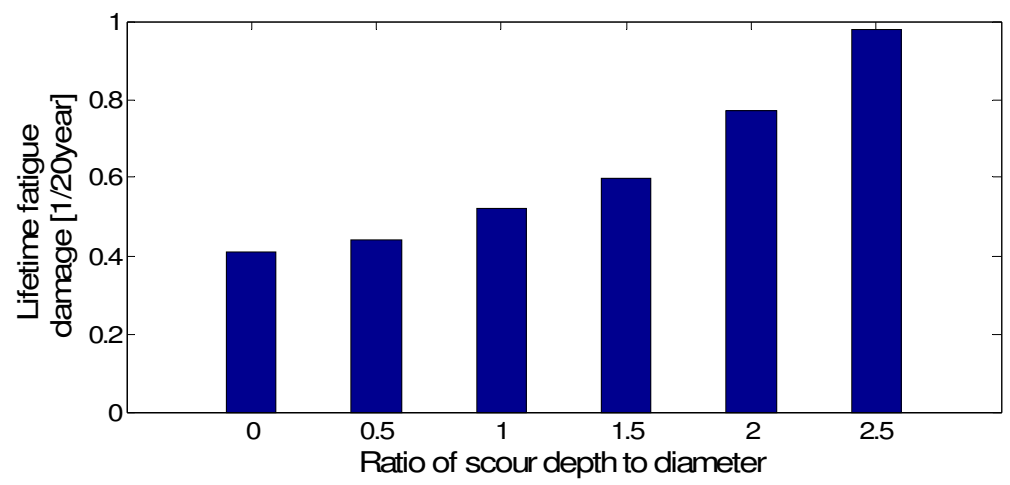

Figure 7.13 Comparison of fatigue damage for varying scour depth

\subsubsection{Conclusions on the impact of scour on fatigue life}

The parameter variation shows that the natural frequency is notably affected by scour depth, albeit that the maximum variation is only $7.5 \%$. The impact on fatigue life, on the other hand, shows much larger differences: more than 100\% increase. The lower natural frequency means that more wave energy can create resonant behaviour of the offshore wind turbine, increasing fatigue damage. The reader is reminded that fatigue damage is governed by the $S$ - $N$ curve with inclined slopes of 3 and 4: small increases in stress level will increase fatigue damage to the power of 3 and 4 .

Although the differences in fatigue damage are very large for these extreme variations in scour depth from $0 D$ to $2.5 D$, the real scour depth is expected to vary between $0.8 D$ and $1.3 D$, as most measurements on real structures have shown [63]. The impact of scour depth on the fatigue damage can be easily assessed with the frequency domain method and dimensions can be optimised to keep the total fatigue damage below the required threshold.

\subsection{Conclusions}

In this chapter the frequency domain fatigue damage calculation method has been applied to a design of the support structure for the OWEZ offshore wind farm. With a natural frequency of $0.31 \mathrm{~Hz}$, this offshore wind turbine is much more prone to fatigue damage than the much stiffer offshore wind turbine at Blyth. For time domain fatigue calculation the total fatigue damage was found to be 0.56 , a fatigue life of 36 years. This excludes the influence of wave diffraction on the wave excitation of the support structure. If diffraction is taken into account the calculated fatigue life increases to 125 years. 
The frequency domain calculated total fatigue damage is entirely comparable to the outcome of the time domain calculations. The following specific conclusions can be drawn:

- reduction of the number of environmental states from 112 to 22 reduces calculation effort in the time domain, and still gives usable results for lifetime fatigue damage

- diffraction correction for the wave excitation significantly reduces lifetime fatigue damage but is not incorporated in the current time domain model; it was therefore not further used in the comparisons of this chapter, but it should always be incorporated in design calculations

- the use of either Rayleigh, Rice or Dirlik is a post-processing choice; Dirlik reportedly produces results that match the outcome of rainflow counting better; however as the execution of the 3 methods is easy and straightforward, it is recommended that all 3 methods are used: Rayleigh and Rice counting methods give a slightly higher lifetime fatigue damage than Dirlik, making the application of either of these methods a good reference for an upper bound of the fatigue damage

- the engineering estimate for aerodynamic damping is a very usable figure for fatigue damage calculations in preliminary design when no turbine details are available

- the Garrad method for aerodynamic damping gave slightly lower fatigue damage compared to the engineering estimate; the method is effective, but the engineering estimate is much more straightforward and does not require any turbine details

- when turbine details are available, the non-linear simulation method gives a much more accurate description of the aerodynamic damping per wind speed bin resulting in even better matching of results of fatigue damage per wind speed bin compared to time domain rainflow counting

- a manual fit of the aerodynamic damping supports the view that the non-linear simulation method may be correct

- more detailed study into the aerodynamic damping is strongly recommended

- current methods for calculating aerodynamic damping are accurate enough for an effective application of the frequency domain fatigue damage calculation method, however

- scour depth variations impact the natural frequency of offshore wind turbine support structures: a scour hole varying from $0 D$ to $2.5 D$ reduces the natural frequency of the OWEZ structure with $7.5 \%$ and the corresponding fatigue damage increases with more than $100 \%$

- the variation of scour depth lies in reality in a much narrower band, making the impact on both natural frequency and fatigue damage much smaller

- frequency domain fatigue damage calculation is fast, accurate and easy to use for fatigue damage calculations. 


\section{Conclusions and Outlook}

\subsection{Conclusions}

\section{General considerations on offshore wind energy}

Offshore Wind Energy has become a reality during the execution of this thesis project from 2001 to 2006. Installation of offshore wind power stations in European waters is common practice with 15 stations running to date. However, the design of the individual offshore wind turbines is still subject to a large number of design uncertainties.

It is a fact that turbines are still standard units, designed to meet typical generalised environmental conditions, these days including "offshore". The turbine itself is not a variable in the design of the offshore wind farm. The support structure on the other hand is a major variable. Support structures vary in size and dimensions from wind farm to wind farm and these days even location specific support structures are being designed within the wind farms. These refinements allow for reduction of cost and improvement of the cost effectiveness of offshore wind.

To achieve further cost reductions, it is imperative to create a widespread understanding of the basics of the design drivers of offshore wind turbines. This thesis aims to assist this understanding and it will be used in the education programme of the Delft University of Technology and probably beyond.

With respect to accelerating the implementation of lessons learned from previous projects, it is striking that the barrier between offshore contractor and turbine manufacturer is still paramount. No matter how effective a contractual split in offshore wind turbine sub-components may seem, the design is certainly not much aided by it. Fortunately, extreme load cases can still be studied rather effectively when turbine and support structure are assessed separately. However, for fatigue a comparison of four offshore wind farm designs shows tremendous variation in approach. The observation in the design report for Horns Rev that "no fatigue damage will be present", based on the evaluation of a single stress amplitude, is a clear example of how things should not be done.

\section{Environment}

The offshore environment needs to be known for the specific site of the offshore wind farm. For most locations, several sources are available: buoys, hind-cast databases and satellite data, of which the latter is becoming a more and more sophisticated source as available data and analysis options increase and the data is tailored to offshore wind design software. 
For the offshore wind turbine site the data needs to be processed into extreme events and combined wind/wave scatter diagrams covering the probability of all environmental states during the lifetime. In this thesis 3-dimensional scatter diagrams were used for mean wind speed, significant wave height and mean zero-crossing wave period. The best way to process these is to make a wave scatter diagram per wind speed bin of $2 \mathrm{~m} / \mathrm{s}$ size. This fits the typical wind speed bins used in turbine design.

For the wave spectral shape several options can be selected. For fatigue calculations, however, most benign sea states are fully developed, justifying a Pierson-Moskowitz representation.

Wave diffraction significantly reduces wave induced loading on the large diameter support structure at the resonant frequency as inertia is the dominant hydrodynamic load component and diffraction reduces the inertia coefficient. Present day monopile support structure dimensions are such that wave diffraction has a significant impact on fatigue life. Existing time domain programs do not readily offer diffraction correction as a modelling option, making the design conservative with the possibility of overconservatism.

For wind loading on the turbine, several theories can be used for determining the various parameters. The theories to use are properly described in the design standards and can be selected according to site-specific data.

\section{General fatigue issues}

With increasing turbine size and weight, and offshore wind farms being planned in increasingly deeper waters, the natural frequency of the support structure will decrease. With a lower natural frequency the structure becomes more and more prone to wave induced fatigue loading. An advantage is that the operating turbine introduces significant aerodynamic damping, decreasing resonant behaviour and consequently fatigue damage.

To calculate the fatigue damage in the design process, the offshore wind turbine is subjected to all possible wind and wave combinations and the stresses at relevant locations in the structure are determined. Furthermore the contribution to the fatigue damage of the pile driving and turbine stops and start-ups are analysed separately. To count the stress cycles to calculate the fatigue damage, several counting methods exist in the time domain and the frequency domain. Peak counting can be used in both time and frequency domain and gives very good results for narrow-band processes such as response to wave loads, but for turbine loads rainflow counting in the time domain is the industry standard. In the frequency domain the Dirlik probability distribution has shown to give the best match with the rainflow counting method.

When calculating fatigue, the simulation parameters should be properly selected, so as not to miss vital information. In the time domain it was found that a time step of $0.25 \mathrm{~s}$ is sufficient for the structures consideration here: 2-3 MW turbines on structures with a 
natural frequency of approximately $0.3 \mathrm{~Hz}$. The computer software used required a smaller time step to prevent aliasing from the internal calculation settings resulting in a selected time step of $0.125 \mathrm{~s}$. For fatigue calculations in single environmental states a duration of $3600 \mathrm{~s}$ has been used with different random seeds for each state. When such a time series is translated to a spectrum the subrecords used to create the spectrum should be long enough to provide a frequency step that is small enough to properly cover the resonance peak. In this case a record length of $512 \mathrm{~s}$ was found to be sufficient. This meant using 512/0.125 $=4096$ points per spectral estimate, which gives, with $50 \%$ overlap, only 14 averages to create a spectrum. To create statistically reliable spectra from time domain simulations preferably 100 averages are needed, requiring a simulation duration of 7.1 hours.

\section{Frequency domain fatigue calculation: uncoupling of turbine and support structure}

The core of the fatigue calculation method presented in this thesis is the full uncoupling of the support structure and the turbine. The turbine is modelled on a rigid support structure (no dynamics) in a regular, fully non-linear time domain program. By running several wind speed cases, transfer functions are derived for turbulent wind speed to tower top loading per wind speed bin, in steps of $2 \mathrm{~m} / \mathrm{s}$ from cut-in to cut-out wind speed. The transfer functions can be used for any support structure configuration and only need to be calculated once.

The support structure can be modelled separately in any finite element program. For this structure two transfer functions are created: from tower top load to bending stress at any location in the structure and from wave height to bending stress at the same location. To solve the equations of motion of the modelled structure, many programs use modal analysis. It is imperative that either of the following options is used: solving all modes of the model or using modal analysis with static back-substitution.

The only interaction between turbine and support structure is the aerodynamic damping, which is calculated separately and incorporated in the model of the support structure as additional viscous damping.

The stress response spectra due to wind loading and due to wave loading are calculated separately and are then added linearly, resulting in the combined stress response spectrum. The Rayleigh, Rice and Dirlik counting methods can be used to determine the distribution of stress variations corresponding with this spectrum in order to calculate the accumulated fatigue damage.

\section{Aerodynamic damping}

Although the existence of aerodynamic damping has been known for many years, it has never been a design parameter. Several theories to derive the magnitude of the aerodynamic damping have been explored. 
Engineering estimate: based on previous experience and to facilitate preliminary design without extensive turbine knowledge an estimated value of $4 \%$ aerodynamic damping was introduced. The estimate gives good results for preliminary design, although the value does not represent variations in aerodynamic damping expected for variable speed, pitch regulated turbines.

Garrad method: a closed form formula to calculate the aerodynamic damping per wind speed bin. This method requires the basic parameters of the wind turbine and is able to incorporate the variable speed option. It can be used to verify the correctness of the engineering estimate for different turbines. The preliminary conclusions on this method are that it gives a reasonable estimate of the damping; it tends to be on the high side compared to the damping estimate using non-linear simulation, which is summarized hereafter.

Non-linear simulation: this method uses the full non-linear time domain model by calculating the variations in rotor thrust due to variations in incident wind speed on the rotor. The method gives a lower estimate than the previous two methods, making it relatively conservative and very useful for detailed design. The drawback of needing a full turbine model can be overcome when turbine manufacturers use this method and supply the wind speed bin dependent aerodynamic damping with their general turbine data.

Aerodynamic damping is a critical factor in the frequency domain fatigue damage calculation method. Although the methods to derive its magnitude vary, the fatigue damage calculated with all methods is within acceptable limits for an effective application of the frequency domain method in preliminary design. As the impact on fatigue is large, more research is required to further the knowledge of the aerodynamic damping and make it an explicit design factor, not only in offshore wind turbine design but also in turbine design and to use the method for detailed design as well.

\section{Comparison to the Blyth offshore wind turbine}

A validated model of the offshore wind turbines at Blyth was available to be used in this thesis. Both the time domain and the frequency domain method were applied to this structure and it was found that the spectra of time and frequency domain calculated stress response match very well. For calculation of fatigue damage the Dirlik method was found to fit the rainflow counted time domain fatigue damage best, although fatigue is not a real issue for these offshore wind turbines as the natural frequency of $0.5 \mathrm{~Hz}$ is too high to result in any significant resonant behaviour from wave action.

Comparison of the frequency domain method to measured stress spectra showed that the method also compares well to the real offshore wind turbine. Perfect agreement will not be possible as the number and length of the measurements is limited and the order of detail of the measured environmental conditions is not high enough to ensure that the 
real and the modelled offshore wind turbine are subjected to exactly the same environmental loads.

\section{Applying the frequency domain method to an OWEZ design}

As the Blyth offshore wind turbine is not susceptible to fatigue, a design for the OWEZ wind farm was used to perform fatigue damage calculations. For this structure fatigue is a design driver. Time domain and frequency domain fatigue damage was calculated for 112 environmental states.

It was found that the lifetime fatigue damage calculated with the frequency domain method matches very well the time domain calculated fatigue damage. The different aerodynamic damping methods were used and it can be concluded that the engineering estimate gives good results for preliminary design and that the non-linear simulation method approaches the time domain fatigue damage results even more closely, including some conservatism. A manual fit of the aerodynamic damping was made to match the frequency domain outcome per wind speed bin to the time domain results. The fitted damping curve supports the basic shape and magnitudes of the non-linear simulation method.

When wave diffraction is correctly applied in the frequency domain a significant decrease of fatigue damage is found when compared to time domain fatigue damage without wave diffraction. It is imperative that for the monopile structures currently being designed, diffraction is considered in the design of the support structure.

Post-processing the frequency domain stress spectra to obtain stress ranges to calculate fatigue damage requires the use of a counting method for the statistical distribution of the stress ranges. Generally, the Dirlik distribution can be expected to provide the closest fit to rainflow counting. However, the Rayleigh and Rice distributions performed equally well and provide an upper bound estimate for fatigue damage. It is therefore recommended that all three distributions are used in practical applications.

To demonstrate the effectiveness of a quick fatigue assessment tool, a parameter variation was carried out for the effect of scour on the natural frequency and the lifetime fatigue damage of the offshore wind turbine. With a variation of scour depth from $0 D$ to $2.5 \mathrm{D}$ the natural frequency decreased $7.5 \%$ and the fatigue damage increased by more than $100 \%$. For real offshore wind turbines the scour depth variation will be much smaller, but the example proved the effectiveness of the frequency domain method to quickly perform such a parameter variation study, which enhances understanding, supports educated design decisions for further optimisation of the offshore wind turbine design and is a powerful tool to reduce its cost. 


\subsection{Outlook}

\section{Future developments}

The method presented in this thesis was carried out with a series of computer programs to produce all required transfer functions and responses. For the purpose of this thesis, further development of the software was not required. For useful application in real projects such a development is vital to streamline the calculation method and make it robust. During the completion of this thesis a project is being carried out at the OWEZ-contractor to apply the frequency domain method to the actual design of the Egmond offshore wind farm. In this project the method is also checked against time domain design calculations and will be included in the software packages of the contractor to make it a standard design tool for future use.

In the offshore industry, the frequency domain fatigue calculation is not only used for preliminary design, but is also the accepted industry standard for final design. The method presented in this thesis can also be used beyond the preliminary design stages although for a final check in integrated time domain models will be remain preferable in the near future. The following will be required to make this feasible:

- development of a calculation method for aerodynamic damping validated using measurements of a number of real offshore wind turbines

- $\quad$ extension of the method to incorporate different wind and wave directions.

\section{Beyond the monopile}

As the turbine size still increases over time and as some offshore wind farms are planned in deeper waters, the monopile should be expected to reach its limit of applicability. Designs of tripods and jackets have already been proposed and will be constructed in pilot projects in the near future. Currently time domain turbine design tools are being extended with modelling techniques to facilitate the design of these structures.

The major advantages of using the frequency domain method are, however:

- its transparency, which promotes better understanding of the underlying processes and provides clearer insight over various design parameters;

- its efficiency in engineering time and computer usage, which makes it possible to investigate the impact of parameter or design variations with the associated potential for design optimisation and cost reduction.

Since the turbine and support structure are uncoupled by separately determining the aerodynamic damping, the frequency domain method as developed in this thesis for monopile structures is generally applicable to any type of support structure. 


\section{References}

[1] Tempel, J van der (2002) Offshore wind, to mill or to be milled ISBN 9076468-09-5, DUWIND, Delft University of Technology

[2] BTM Consult ApS (2005) International Wind Energy Development, World Market update 2004, Forecast 2005-2009 IC Christensens Allé 1, DK6950 Ringkøbing, Denmark

[3] Camp, T et al. (2003) Design Methods for Offshore Wind Turbines at Exposed Sites, final report. Garrad Hassan and partners, 2317/BR/22D

[4] Kühn, M (2001) Dynamics and Design Optimisation of Offshore Wind Energy Conversion Systems Institute for Wind Energy, Delft University of Technology ISBN 90-76468-07-9

[5] Ferguson, MC (ed.) et al. (1998) Opti-OWECS Final Report Vol.4: A typical Design Solution for an Offshore Wind Energy Conversion System Institute for Wind Energy, Delft University of Technology

[6] Bendat, JS and Piersol, AG (1980) Engineering applications of correlation and spectral analysis A Wiley-Interscience Publication, New York

[7] Vugts, JH (1995) Stochastic Processes Lecture notes for X2CT3, Offshore Engineering, Delft University of Technology

[8] Pierson, WJ, Moskowitz, LA (1964) A proposed spectral form of fully developed wind seas based on the similarity theory of S.A. Kitaigorodshii, J. Geopys. Res., Vol. 69

[9] Hasselmann, et al, (1973) Measurements of wind wave growth and swell decay during the Joint North Sea Wave Project Deutsche Hydro. Zeitschr. Riehe, A8

[10] Chakrabarti S.K. (1987) Hydrodynamics of Offshore Structures ISBN 0905451-66-X Computational Mechanics Publications Southampton

[11] Wheeler, JD (1970) Method for Calculating Forces Produced by Irregular Waves, J. of Petroleum Technology, pp. 359-367

[12] ISO 19901-1 (2005) Petroleum and natural gas industries - Specific requirements for offshore structures - Part 1: Metocean design and operating considerations International Organization for Standardization, Geneva, Switzerland

[13] Eng-Soon, C et al. (1994) Laboratory study of plunging wave impacts on vertical cylinders Department of Civil Engineering, National University of Singapore

[14] Hagemeijer, PM (1985) Breaking waves and wave forces in shallow water: a review Koninklijke/Shell Exploratie en Produktie Laboratorium

[15] HMSO for tides, waves etc.

[16] www.wmo.ch 
[17] Hoven, I van der (1957) Power spectrum of horizontal wind speed in the frequency range of 0.0007 to 900 cycles per hour Journal of Meterology $14 \mathrm{pp}$ 160-4

[18] Unknown participant (2004) Comment during IEA expert meeting on offshore wind energy, Ringkøbing Denmark

[19] DNV (2004) Design of offshore wind turbine structures Det Norske Veritas, DNV-OS-J101

[20] Germanischer Lloyd (2000), Rules \& Guidelines 2000: IV Non-marine Technology - Regulations for the Certification of (Offshore) Wind Energy Conversion Systems

[21] Burton, T et al., (2001) Wind Energy Handbook, John Wiley \& Sons Ltd, England, ISBN 0-471-48997-2

[22] IEC (1999) Wind turbine generator systems - Part 1: Safety requirements International standard 61400-1, International Electrotechnical Commission

[23] DS472 (1992) Code of practice for loads and safety of wind turbine constructions Danish Society of Engineers and the Federation of Engineers

[24] www.waveclimate.com

[25] Wieringa, J (1973) Gust factor over open water and built-up country Boundary layer Meterology, 3 pp 424-441

[26] Matthies, HG, et al (1995) Study of Offshore Wind Energy in the EC, Joule I (JOUR 0072) Verlag Natürliche Energie, Brekendorf

[27] www.hornsrev.dk

[28] Manwell, JF, McGowan, JG, Rogers, AL (2002) Wind energy explained John Wiley \& Sons Ltd, Baffins Lane, Chichester, West Sussex PO19 1UD Dengland, ISBN 0-470-84612-7

[29] Vestas (2003) V80-2MW OptiSpeed ${ }^{T M}$ Offshore Wind Turbine leaflet on www.vestas.dk

[30] Betz, A (1920) Das maximum der theoretisch möglichen Auswendung des Windes durch Windmotoren Zeitschrift für gesamte Turbinewesen, vol. 26, p 307

[31] Beurskens, J, Kuik, G. van (2004) Alles in de wind, vragen en antwoorden over windenergie, Daedalus, Maastricht, ISBN 90-71580-02-4

[32] Molenaar, D-P, Dijkstra Sj (1999) Modelling the structural dynamics of flexible wind turbines. In Proceedings of European Wind Energy Conference and Exhibition, Acropolis Convention Centre, Nice, France

[33] Vugts, JH (2000) Considerations on the dynamics of support structures for an OWEC Section Offshore Technology, Delft University of Technology

[34] Tempel, J van der (2000) Lifetime Fatigue of an Offshore wind Turbine Support Structure Section Offshore Technology \& Section Wind Energy, Delft University of Technology

[35] Divone, L (1984) from: Jos Beurskens' personal archives 
[36] Verburg, WH (1996) (Over) Spannend Staal Overspannend Staal - Rotterdam, ISBN 90-72830-18-0

[37] Miner, MA (1945) Cumulative damage in fatigue Trans. ASME, Journal of Applied Mechanics, Vol. 67, pp. A159-A164, 1945.

[38] AMEC (1997) Design document for Blyth offshore wind farm

[39] Kühn M (1998) Simplified Dynamics Analysis of a Monopile Support Structure for the TW $1.5 \mathrm{~s}$ at a Baltic Site, Institute for Wind Energy, Delft University of Technology

[40] LIC Engineers A/S (1996) Mono Pile Foundation

[41] Kempers, M (2003), Design for OWEZ, IC + E

[42] HMC (2002) Basis for Design of the Ringhorne Jacket, Heerema Marine Contractors

[43] Cheng, P (2002) A Reliability Based Design Methodology for Extreme Responses of Offshore Wind Turbines, PhD. Thesis, Delft University of Technology, Section Wind Energy

[44] Unkown speaker at Washington workshop (2003)

[45] Engelen, T, Oei, T, Rieffe, H, Dragt, J (1991) TURBU-I Computer Program for Turbulence Load Analysis of Horizontal Axis Wind Turbines, ECN-I--91-073, ECN, Petten, The Netherlands

[46] Rademakers, LWMM, Werff, PA van der (1992) Verification of wind turbine design codes wit the "Dutch handbook wind data for wind turbine design" ECNI-92-048 ECN, Petten, The Netherlands

[47] Camp, TR (2003) Verification of design tools Garrad Hassan and Partners, document no. 2317/BR/16, OWTES under EU contract JOR3-CT95-0284

[48] Hofland, LD (1999) Fatigue of the support structure of an OWEC due to the combined loading of wind and waves Thesis Main Report, Delft University of Technology, Faculty of Civil Engineering and Geoscience, Section Offshore Technology

[49] Freris, L.L. (1990) Wind Energy Conversion Systems ISBN 0-13-960527-4 Prentice Hall International, UK

[50] Morris, MJ (2003) Description of database of measurements Garrad Hassan and Partners, document no. 2317/BR/20, OWTES under EU contract JOR3-CT950284

[51] Camp, TR (2003) Verification of design tools Garrad Hassan and Partners, document no. 2317/BR/16, OWTES under EU contract JOR3-CT95-0284

[52] API (2000) Recommended Practice for Planning, Design and Constrcucting Fixed Offshore Platforms - Working Stress Design American Petroleum Institute, $21^{\text {st }}$ edition 
[53] Tempel, J van der (ed.) et al. (2003) Robustness of Design Load Calculations for Offshore Wind Turbines OWTES task 4.3, EW-03191, DUWIND, Delft University of Technology

[54] Bendat, JS and Piersol, AG (1986) Random data: Analysis and measurement procedures $2^{\text {nd }}$ Edition A Wiley - Interscience Publication, New York

[55] Cerda Salzmann, D (2003) Dynamic Response Calculation of Offshore Wind Turbine Monopile Support Structures Offshore Engineering, Delft University of Technology

[56] Fugro-GEOS (1999) Metocean criteria for the Blyth offshore wind farm

[57] Zaaijer, MB (2000) Sensitivity analysis for foundations of offshore wind turbines, Section Wind Energy, WE 02181, Delft

[58] Shaw, VK (2004) Foundation Model and Design for Offshore Wind Turbine Monopiles, Offshore Engineering, Delft University of Technology

[59] Bundesambt für Seeschiffart und Hydrographie (1996) Ostsee-Handbuch Teil B: Natverhältnisse Nr. 2003, Hamburg, 18 November 1996

[60] Engelen, TG, Braam, H. (2004) TURBU Offshore, computer program for frequency domain analysis of horizontal axis offshore wind turbines, ECN, ECN-C--04079

[61] STABCON (2004) Intermediate report on aero-elastic instabilities: code comparison

[62] Ippen, AZ (editor) (1966) Estuary and Coastline Hydrodynamics; Engineering Society Monographs, McGraw Hill Book Company, chapter 8.1.

[63] Tempel, J van der, Zaaijer, MB, Subroto, TS (2004) The effects of scour on the design of offshore wind turbines, proceedings of MAREC 2004, Blyth UK

[64] Germanischer Lloyd (2004) Rules and Guidelines for the Design of Offshore Wind turbines, Hamburg, Germany

[65] IEC 16400-3 (2006) Design requirements for Offshore Wind Turbines (Draft)

[66] Lysen, EH (1983) Introduction to Wind Energy, SWD Publications SWD 82-1, The Netherlands

[67] DNV (2002) SESAM Framework manual Det Norske Veritas, report no. 92-7050

[68] Matlock, H (1970) Correlations for Design of Laterally Loaded Piles in Soft Clay, Paper number OTC 1204, Proceedings Second Annual Offshore Technology Conference, Houston, Texas, USA, pp. 577-594

[69] Barltrop, NDP, Adams, AJ (1991) Dynamics of Fixed Marine Structures, Butterworth-Heinemann Ltd, Linacre House, Oxford, ISBN 0750610468

[70] Matsuiski, M, Endo, T (1969) Fatigue of metals subjected to varying stress, Japan Soc. Mech. Eng.

[71] Dijk, GM van, Jonge, JB de (1975) Introduction to a fighter aircraft loading standard for fatigue evaluation - FALSTAFF, National Aerospace Laboratory, NLR, MP75017U, Amsterdam, Holland 
[72] Rice, SO (1944) Mathematical analysis of random noise. Bell System Technical Journals, vol. 23-24.

[73] Vugts, JH (1973) Short- and long-term statistics in a probabilistic approach to waves and marine structures O.D.C. report 39, Shell Oil Company, Southern E\&P Region

[74] Dirlik, T (1985) Application of computers in fatigue analysis Ph.D. Thesis, Warwick University

[75] Benasciutti, D, Tovo, R (2005) Spectral methods for lifetime prediction under wide-band stationary random processes, International Journal of fatigue, $27 \mathrm{pp}$. 867-877

[76] ISO 19901-4 (2003) Petroleum and natural gas industries specific requirements for offshore structures - Part 4: geotechnical and foundation design considerations Issued 2003-08-01 by the International Standards Organization

[77] ISO 19902 DIS (2004) Petroleum and natural gas industries specific requirements for fixed offshore structures Issued 2004-09-30 by the International Standards Organization

[78] Camp, TR (2005) e-mail communications on fatigue settings

[79] Lutz K (2005) e-mail communications on fatigue settings

[80] Cerda Salzmann D, Tempel J van der (2005) Aerodynamic damping in the design of support structures for offshore wind turbines, Proceedings of the Offshore Wind Energy Conference, Copenhagen, Denmark

[81] Waegter, J (2005) email communication on fatigue settings

[82] DNV (2005) email communication on fatigue settings

[83] GL (2005) email communication on fatigue settings

[84] Vries, W de (2005) Time domain fatigue analysis for an offshore wind turbine design for the Nearshore Wind Farm, Offshore Engineering, Delft University of Technology 


\section{Appendix I}

\section{Wave induced inertia and drag load and moment graphs}
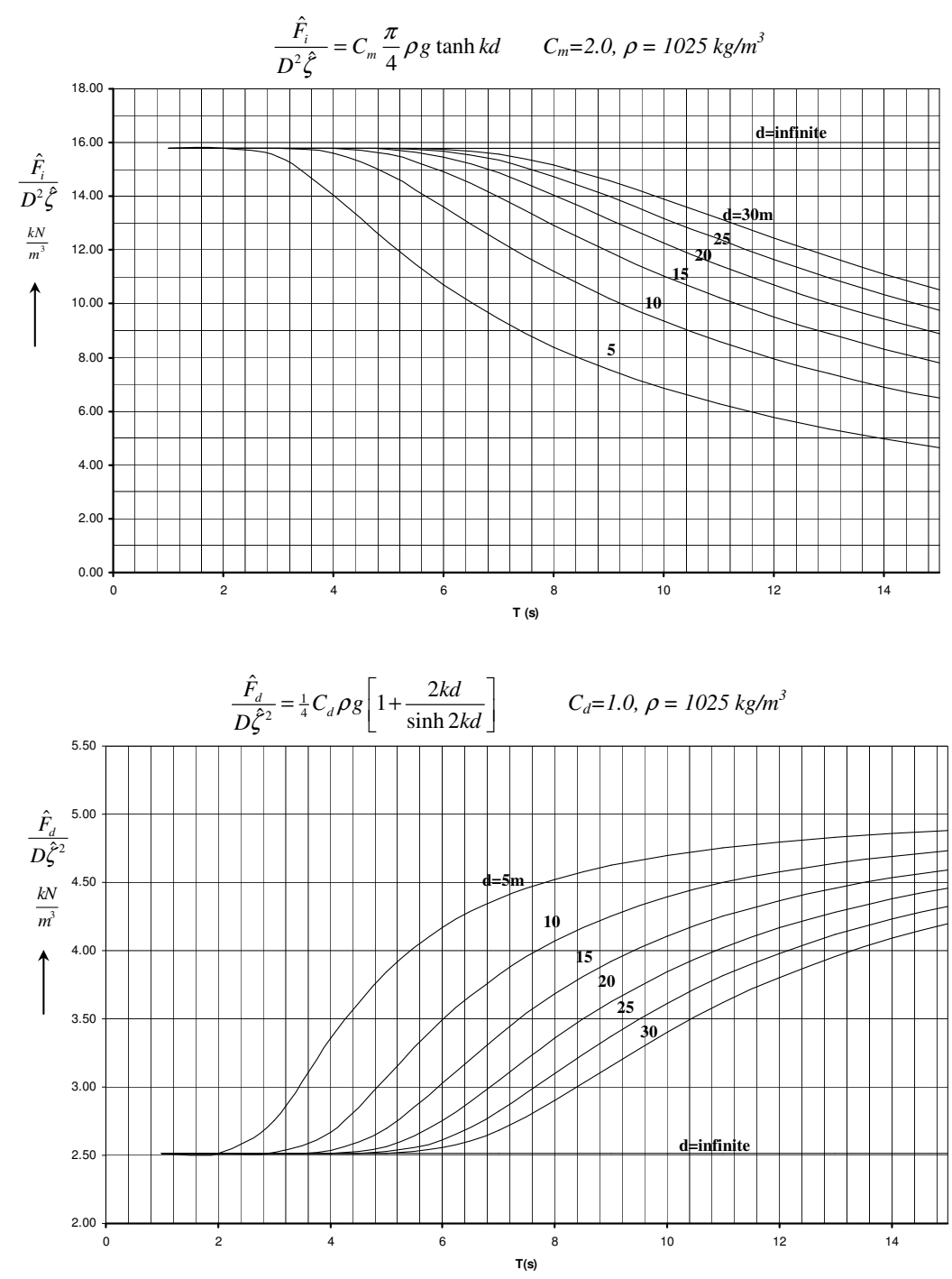

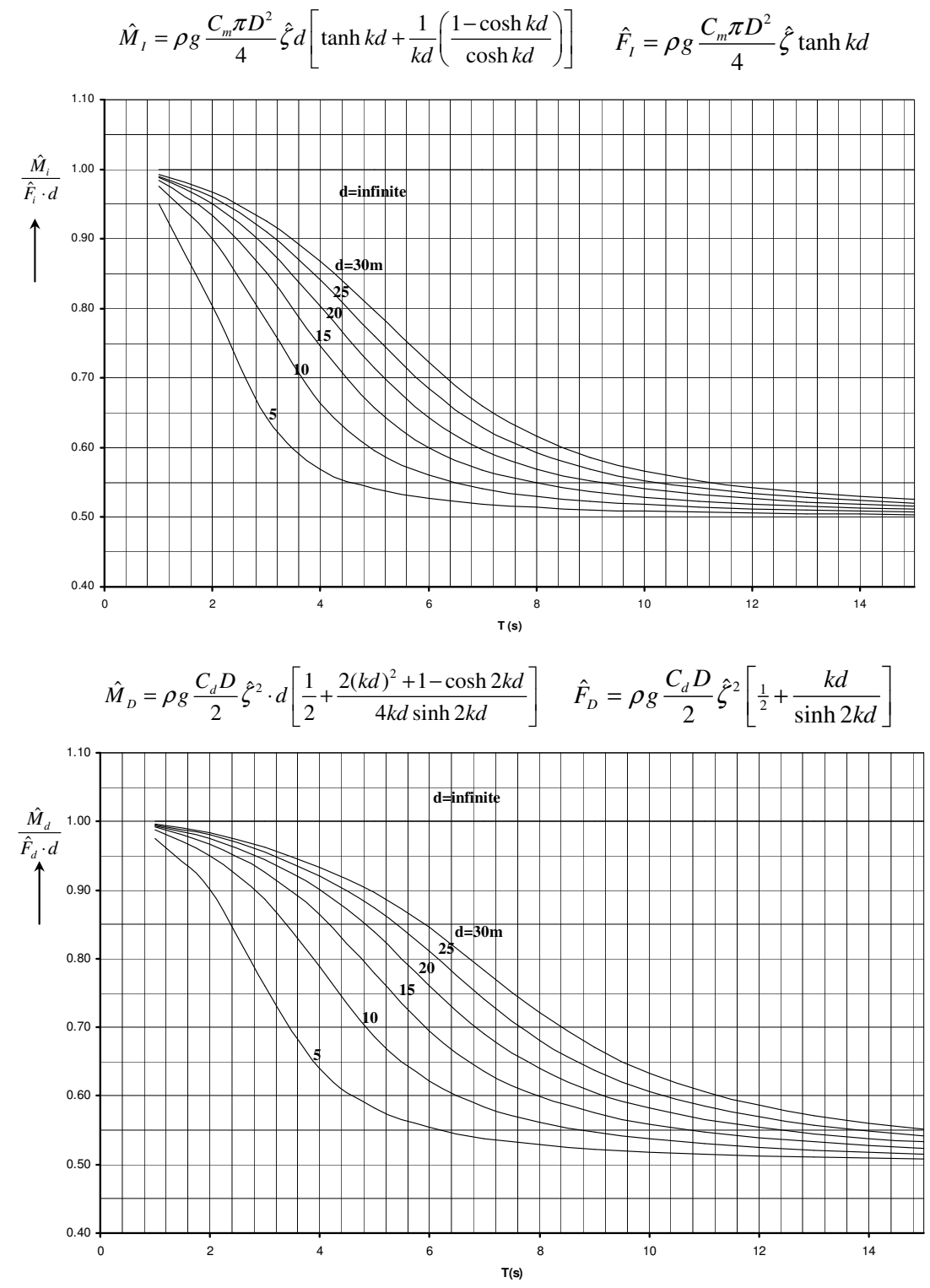


\section{Appendix II 3D scatter diagram for OWEZ site}
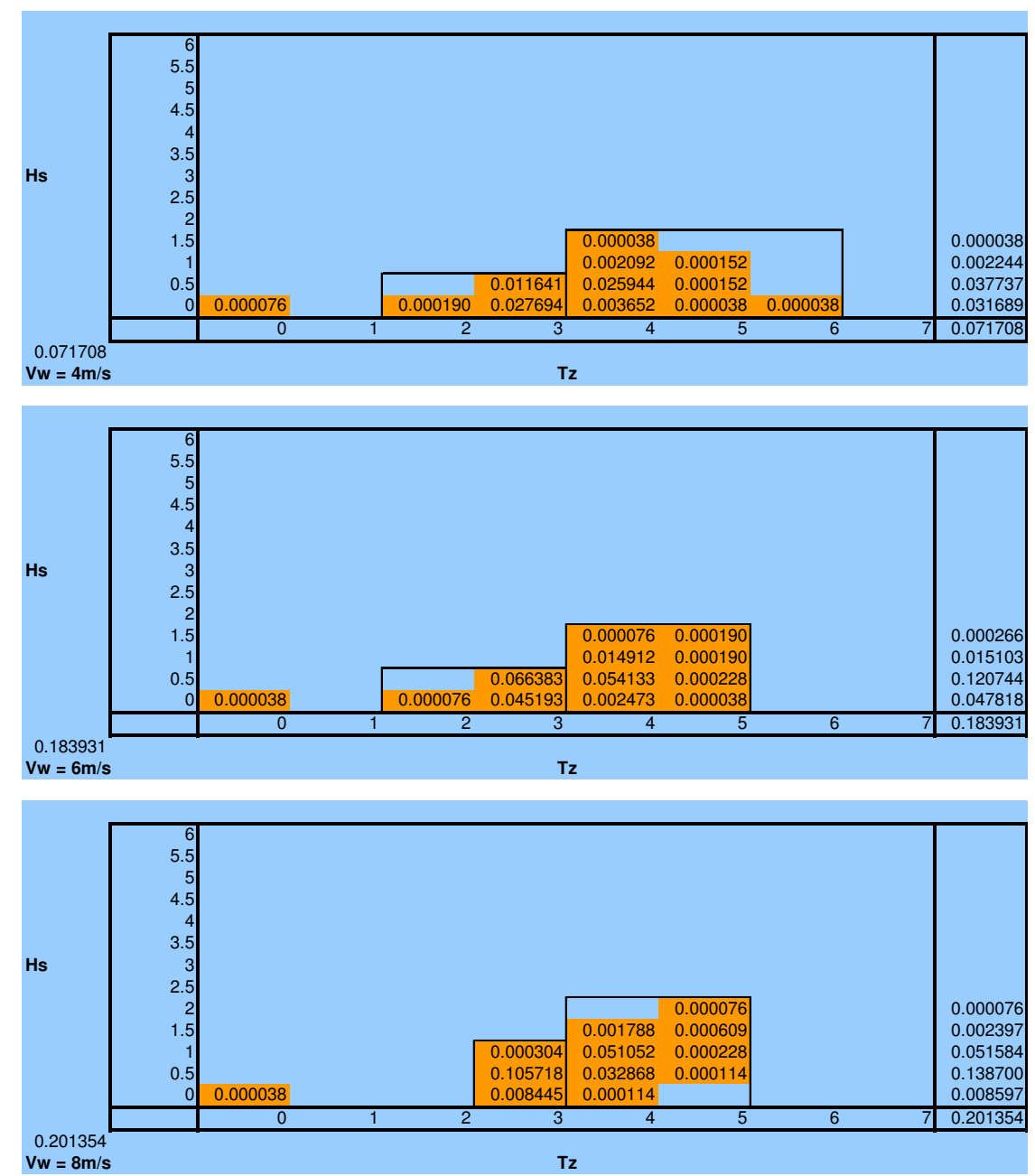

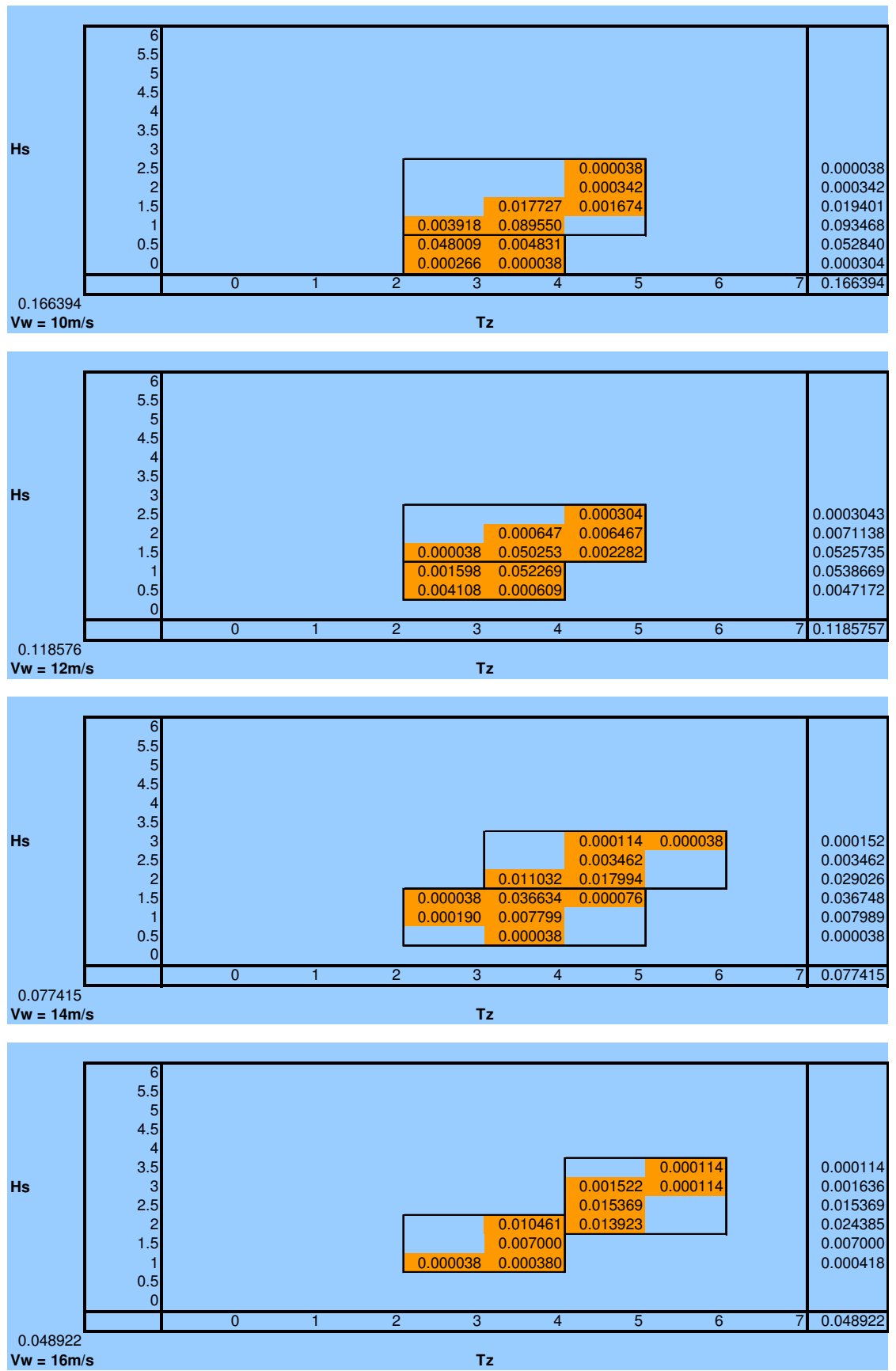

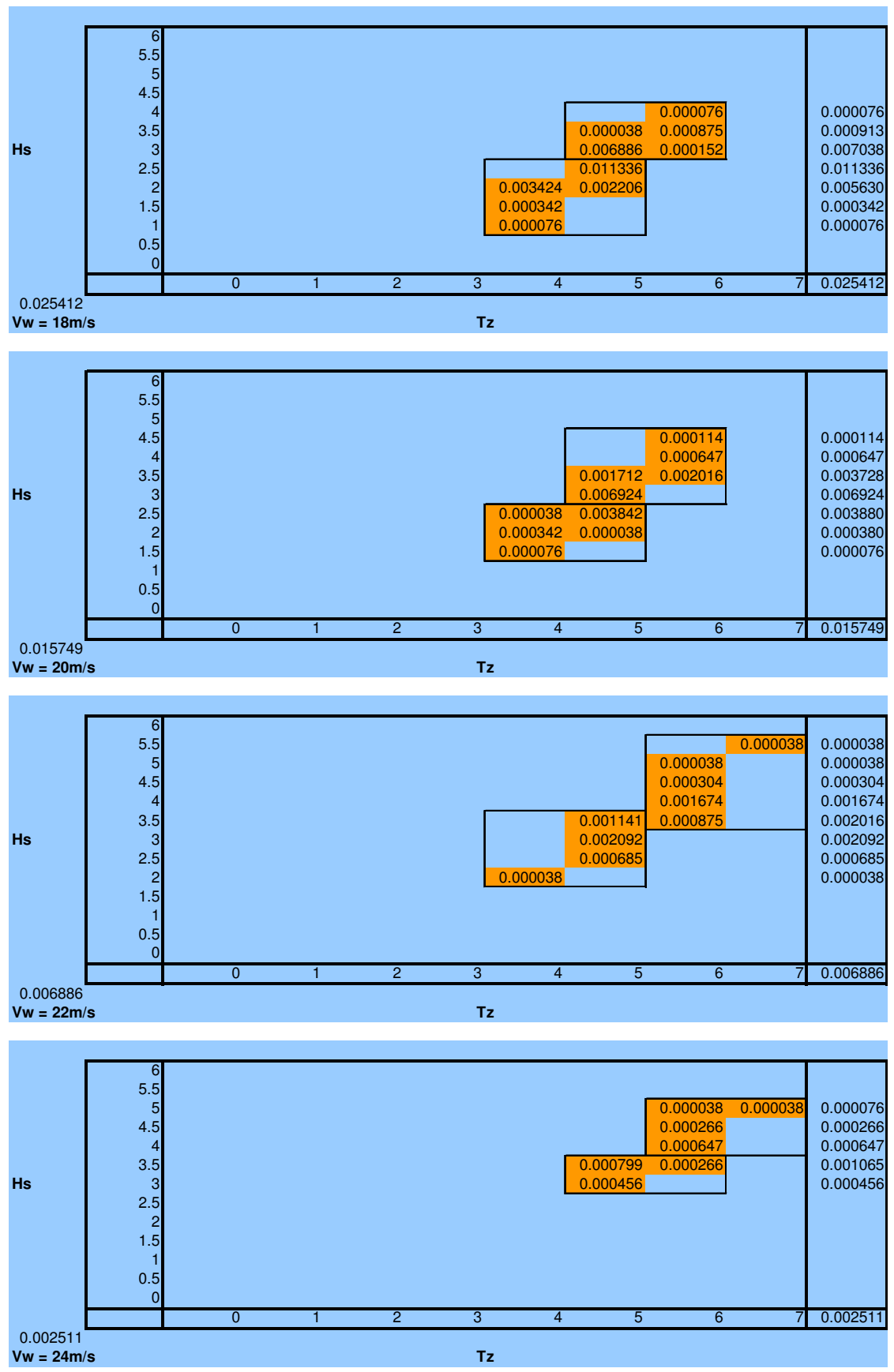


\section{Appendix III Parameters of all 112 states}

\begin{tabular}{|c|c|c|c|c|}
\hline State & $\mathrm{Vw}$ & $\mathrm{Tz}$ & $\mathrm{Hs}$ & $\%$ \\
\hline 1 & 4 & 0.1 & 0 & $0.008 \%$ \\
\hline 2 & 4 & 2 & 0 & $0.019 \%$ \\
\hline 3 & 4 & 3 & 0 & $2.769 \%$ \\
\hline 4 & 4 & 3 & 0.5 & $1.164 \%$ \\
\hline 5 & 4 & 4 & 0 & $0.365 \%$ \\
\hline 6 & 4 & 4 & 0.5 & $2.594 \%$ \\
\hline 7 & 4 & 4 & 1 & $0.209 \%$ \\
\hline 8 & 4 & 4 & 1.5 & $0.004 \%$ \\
\hline 9 & 4 & 5 & 0 & $0.004 \%$ \\
\hline 10 & 4 & 5 & 0.5 & $0.015 \%$ \\
\hline 11 & 4 & 5 & 1 & $0.015 \%$ \\
\hline 12 & 4 & 6 & 0 & $0.004 \%$ \\
\hline 13 & 6 & 0.1 & 0 & $0.004 \%$ \\
\hline 14 & 6 & 2 & 0 & $0.008 \%$ \\
\hline 15 & 6 & 3 & 0 & $4.519 \%$ \\
\hline 16 & 6 & 3 & 0.5 & $6.638 \%$ \\
\hline 17 & 6 & 4 & 0 & $0.247 \%$ \\
\hline 18 & 6 & 4 & 0.5 & $5.413 \%$ \\
\hline 19 & 6 & 4 & 1 & $1.491 \%$ \\
\hline 20 & 6 & 4 & 1.5 & $0.008 \%$ \\
\hline 21 & 6 & 5 & 0 & $0.004 \%$ \\
\hline 22 & 6 & 5 & 0.5 & $0.023 \%$ \\
\hline 23 & 6 & 5 & 1 & $0.019 \%$ \\
\hline 24 & 6 & 5 & 1.5 & $0.019 \%$ \\
\hline 25 & 8 & 0.1 & 0 & $0.004 \%$ \\
\hline 26 & 8 & 3 & 0 & $0.845 \%$ \\
\hline 27 & 8 & 3 & 0.5 & $10.572 \%$ \\
\hline 28 & 8 & 3 & 1 & $0.030 \%$ \\
\hline & & & \\
\hline & & & \\
\hline & & &
\end{tabular}

\begin{tabular}{|c|c|c|c|c|}
\hline State & $\mathrm{Vw}$ & $\mathrm{Tz}$ & $\mathrm{Hs}$ & $\%$ \\
\hline 57 & 14 & 3 & 1 & $0.019 \%$ \\
\hline 58 & 14 & 3 & 1.5 & $0.004 \%$ \\
\hline 59 & 14 & 4 & 0.5 & $0.004 \%$ \\
\hline 60 & 14 & 4 & 1 & $0.780 \%$ \\
\hline 61 & 14 & 4 & 1.5 & $3.663 \%$ \\
\hline 62 & 14 & 4 & 2 & $1.103 \%$ \\
\hline 63 & 14 & 5 & 1.5 & $0.008 \%$ \\
\hline 64 & 14 & 5 & 2 & $1.799 \%$ \\
\hline 65 & 14 & 5 & 2.5 & $0.346 \%$ \\
\hline 66 & 14 & 5 & 3 & $0.011 \%$ \\
\hline 67 & 14 & 6 & 3 & $0.004 \%$ \\
\hline 68 & 16 & 3 & 1 & $0.004 \%$ \\
\hline 69 & 16 & 4 & 1 & $0.038 \%$ \\
\hline 70 & 16 & 4 & 1.5 & $0.700 \%$ \\
\hline 71 & 16 & 4 & 2 & $1.046 \%$ \\
\hline 72 & 16 & 5 & 2 & $1.392 \%$ \\
\hline 73 & 16 & 5 & 2.5 & $1.537 \%$ \\
\hline 74 & 16 & 5 & 3 & $0.152 \%$ \\
\hline 75 & 16 & 6 & 3 & $0.011 \%$ \\
\hline 76 & 16 & 6 & 3.5 & $0.011 \%$ \\
\hline 77 & 18 & 4 & 1 & $0.008 \%$ \\
\hline 78 & 18 & 4 & 1.5 & $0.034 \%$ \\
\hline 79 & 18 & 4 & 2 & $0.342 \%$ \\
\hline 80 & 18 & 5 & 2 & $0.221 \%$ \\
\hline 81 & 18 & 5 & 2.5 & $1.134 \%$ \\
\hline 82 & 18 & 5 & 3 & $0.689 \%$ \\
\hline 83 & 18 & 5 & 3.5 & $0.004 \%$ \\
\hline 84 & 18 & 6 & 3 & $0.015 \%$ \\
\hline
\end{tabular}

\begin{tabular}{|c|c|c|c|c|}
\hline State & $\mathrm{Vw}$ & $\mathrm{Tz}$ & $\mathrm{Hs}$ & $\%$ \\
\hline 85 & 18 & 6 & 3.5 & $0.087 \%$ \\
\hline 86 & 18 & 6 & 4 & $0.008 \%$ \\
\hline 87 & 20 & 4 & 1.5 & $0.008 \%$ \\
\hline 88 & 20 & 4 & 2 & $0.034 \%$ \\
\hline 89 & 20 & 4 & 2.5 & $0.004 \%$ \\
\hline 90 & 20 & 5 & 2 & $0.004 \%$ \\
\hline 91 & 20 & 5 & 2.5 & $0.384 \%$ \\
\hline 92 & 20 & 5 & 3 & $0.692 \%$ \\
\hline 93 & 20 & 5 & 3.5 & $0.171 \%$ \\
\hline 94 & 20 & 6 & 3.5 & $0.202 \%$ \\
\hline 95 & 20 & 6 & 4 & $0.065 \%$ \\
\hline 96 & 20 & 6 & 4.5 & $0.011 \%$ \\
\hline 97 & 22 & 4 & 2 & $0.004 \%$ \\
\hline 98 & 22 & 5 & 2.5 & $0.068 \%$ \\
\hline 99 & 22 & 5 & 3 & $0.209 \%$ \\
\hline 100 & 22 & 5 & 3.5 & $0.114 \%$ \\
\hline 101 & 22 & 6 & 3.5 & $0.087 \%$ \\
\hline 102 & 22 & 6 & 4 & $0.167 \%$ \\
\hline 103 & 22 & 6 & 4.5 & $0.030 \%$ \\
\hline 104 & 22 & 6 & 5 & $0.004 \%$ \\
\hline 105 & 22 & 7 & 5.5 & $0.004 \%$ \\
\hline 106 & 24 & 5 & 3 & $0.046 \%$ \\
\hline 107 & 24 & 5 & 3.5 & $0.080 \%$ \\
\hline 108 & 24 & 6 & 3.5 & $0.027 \%$ \\
\hline 109 & 24 & 6 & 4 & $0.065 \%$ \\
\hline 110 & 24 & 6 & 4.5 & $0.027 \%$ \\
\hline 111 & 24 & 6 & 5 & $0.004 \%$ \\
\hline 112 & 24 & 7 & 5 & $0.004 \%$ \\
\hline
\end{tabular}




\section{Appendix IV \\ Comparison of response spectra for time domain and frequency domain for 112 \\ states for manually fitted aerodynamic damping}



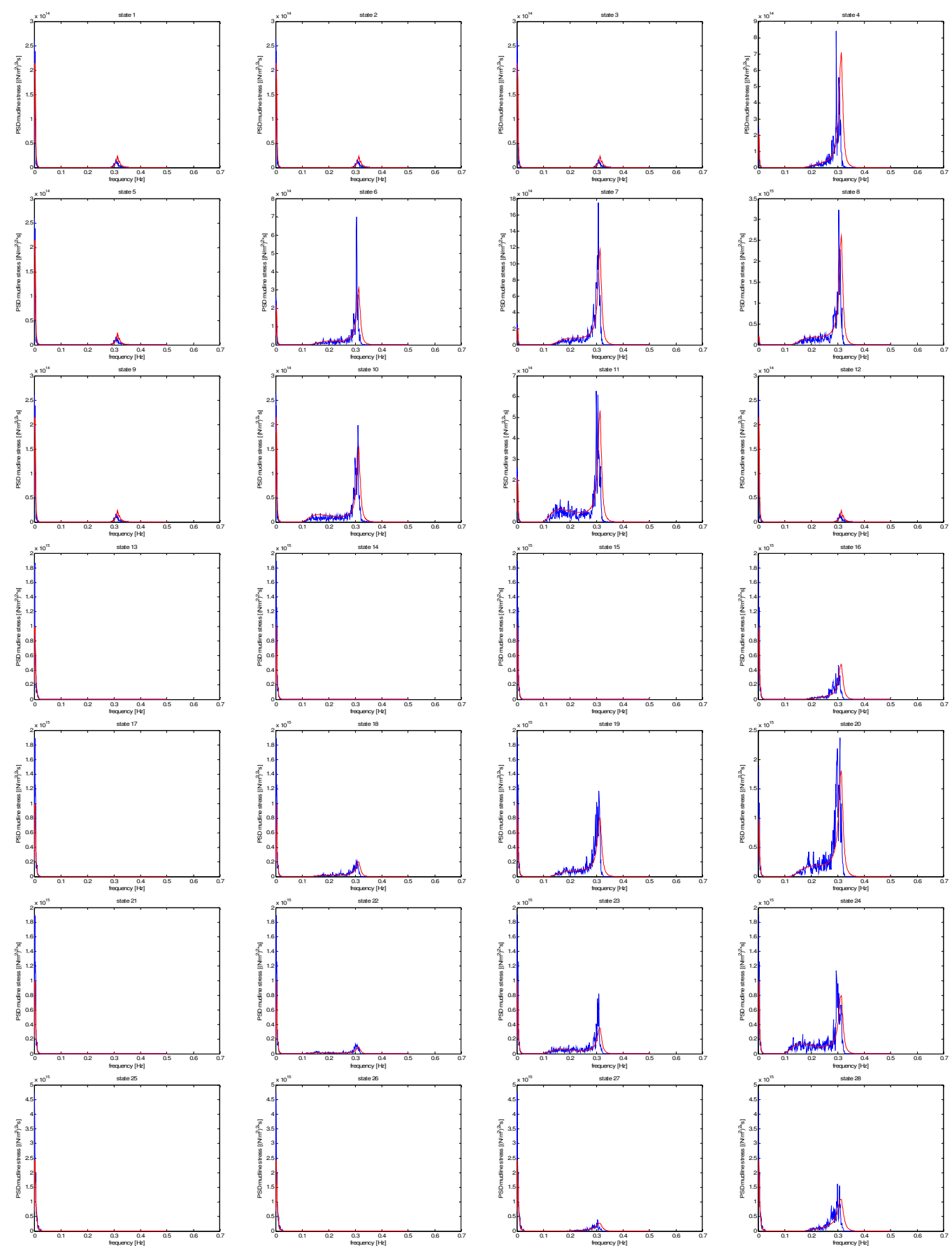

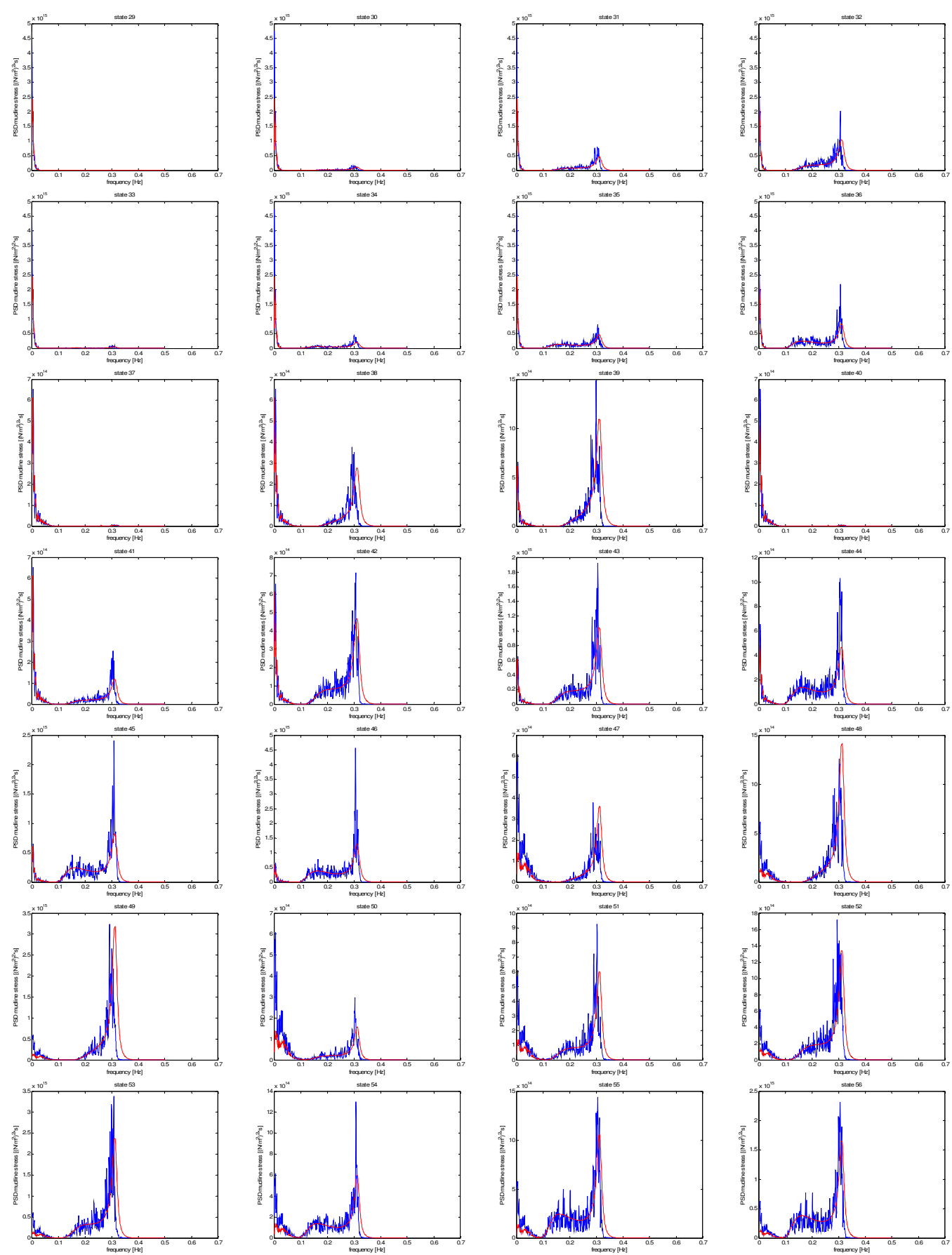

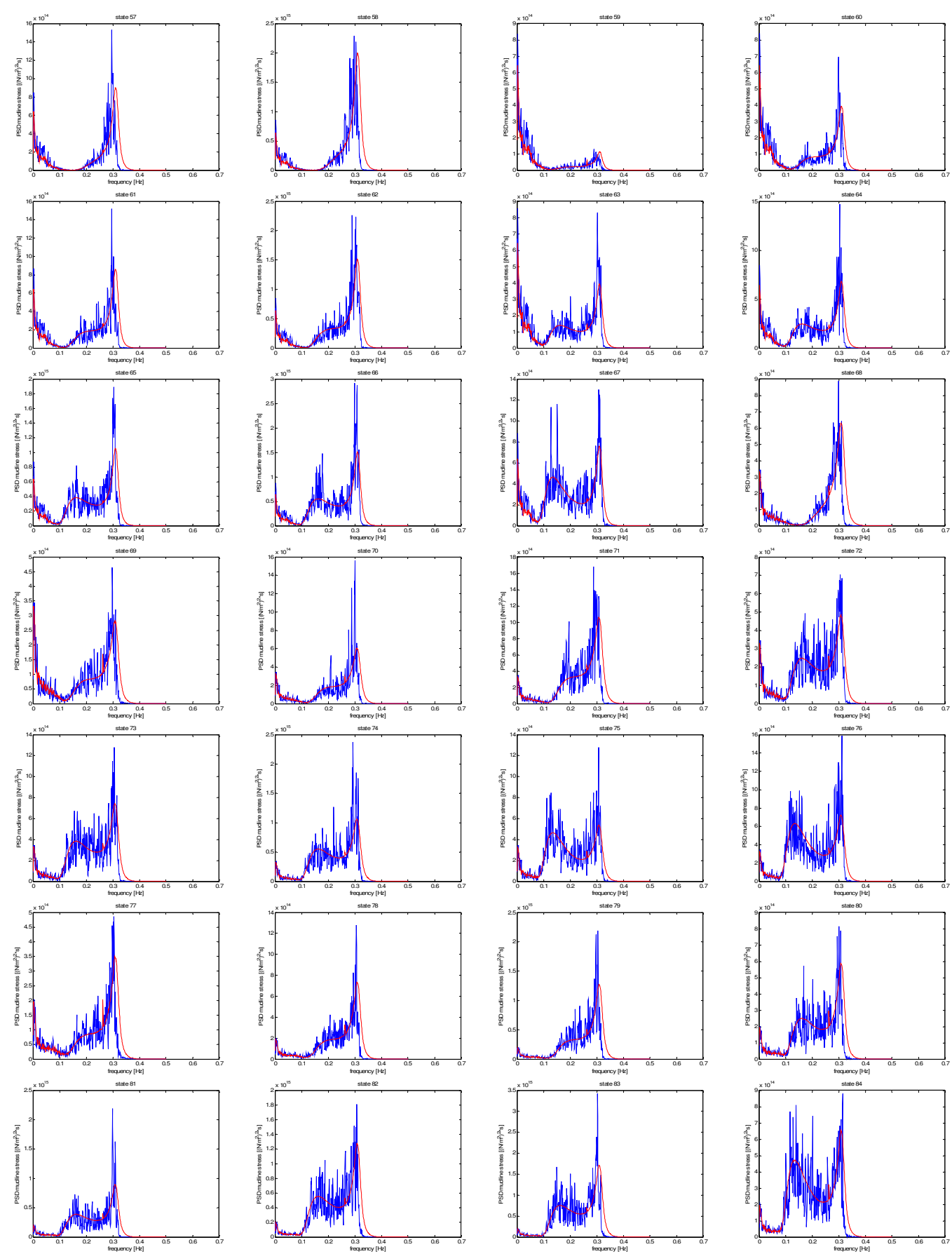

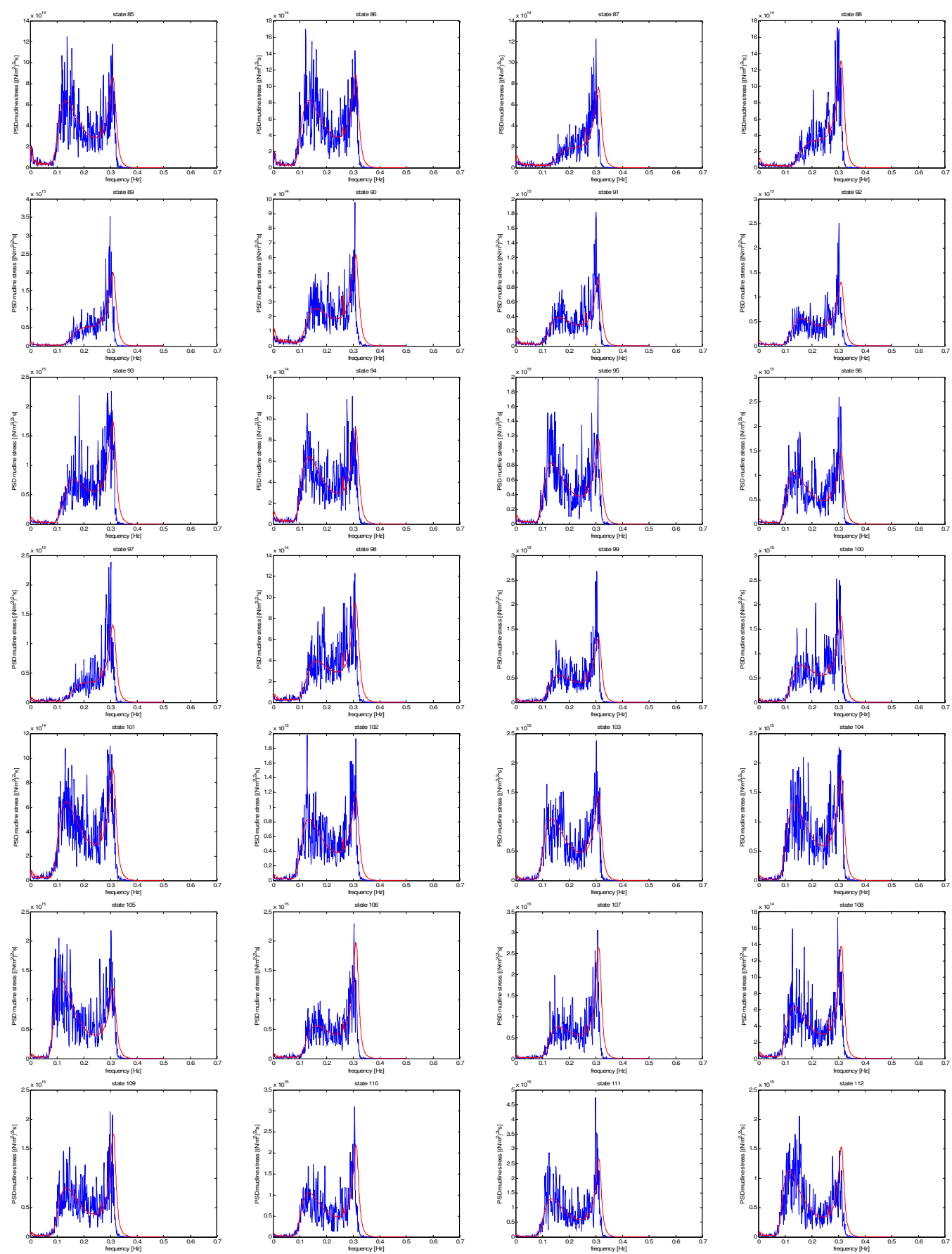


\section{Samenvatting}

Door de groeiende vraag naar energie, het Kyoto protocol en de gewenste diversificatie van energiebronnen is windenergie uitgegroeid tot een volwassen industrie, met een kostprijs van elektriciteit die al kan concurreren met gasgestookte centrales. Om ruimte te bieden aan nog meer windvermogen zijn de turbines het afgelopen decennium buitengaats gegaan. Op zee is de windsnelheid hoger en de kwaliteit van het windaanbod beter dan op land, wat resulteert in een hogere elektriciteitsopbrengst. Aan de andere kant zijn de kosten van het installeren van offshore windturbines hoger dan op land.

Om de rendabiliteit van offshore wind te verbeteren moeten de risico's goed in kaart gebracht en verminderd worden en moeten de kritische ontwerpfactoren worden geoptimaliseerd. Vanuit het ontwerp gezien vraagt dit om de volgende stappen:

- het doorgronden van de werking van offshore windturbines

- het toepassen van kennis opgedaan in voorgaande projecten

- het verbeteren van ontwerpgereedschappen.

Dit proefschrift richt zich op het ontwerp van de ondersteuningsconstructie. Eerst worden de basisprincipes van het ontwerpen van traditionele offshore constructies en van windturbines samengevat, met nadruk op het toepassen van deze principes op de ondersteuningsconstructie. Vervolgens wordt een overzicht gegeven van de ontwerpen van vier offshore windparken en een offshore platform. De ontwerpmethoden zijn onderling vergeleken en wezen uit dat de methodologie voor het merendeel consistent is. Alleen de aanpak van de berekening van de vermoeiingsschade verschilt van project tot project.

In de offshore olie- en gasindustrie wordt vermoeiingsschade berekend in het frequentiedomein. Dit kan omdat de golfbelastingen gelineariseerd kunnen worden. De voordelen van het frequentiedomein zijn de inzichtelijkheid van de tussen- en eindantwoorden alsmede de snelheid van de berekeningen. In de windindustrie (zowel onshore als offshore) is het gebruikelijk vermoeiingsberekeningen in het tijddomein uit te voeren om zodoende alle niet-lineaire effecten van de turbine in rekening te kunnen brengen. Een nadeel van deze methode voor het ontwerp van ondersteuningsconstructies is dat de offshore aannemer vaak te weinig kennis heeft van aërodynamica en van de details van de turbine om een volledig model te kunnen maken in het tijddomein. In dit proefschrift is een frequentiedomein methode ontwikkeld die dit probleem ondervangt. Bij deze methode is de uitwisseling van informatie over de turbine dusdanig beperkt dat de turbinefabrikant geen gevoelige informatie hoeft prijs te 
geven aan de offshore aannemer om toch tot een goed ontwerp van de ondersteuningsconstructie te komen. De offshore ontwerper kan vervolgens de ondersteuningsconstructie optimaliseren met behulp van zijn vertrouwde frequentiedomein software.

De frequentiedomein methode is getest op het ontwerp van de offshore windturbines bij Blyth, waarvan een gevalideerd computermodel en offshore metingen beschikbaar waren. Verder is de methode toegepast op een ontwerp van het offshore windpark dat bij Egmond zal verschijnen in 2006. In beide gevallen bleek de frequentiedomein methode heel goed te werken en resultaten te geven die goed overeenkomen met de uitkomsten van de tijddomein simulaties. De benodigde rekentijd voor een vermoeiingscheck is gereduceerd van meerdere uren in het tijddomein tot minder dan 2 minuten in het frequentiedomein. Deze snelheid van rekenen maakt het mogelijk door parametervariatie de gevoeligheid van het ontwerp voor verschillende ontwerpkeuzes te toetsen en voor elke constructie in een windpark een specifieke geometrie te ontwerpen.

Een cruciaal onderdeel van de methode is de invloed van de aërodynamische demping die de draaiende turbine introduceert op de dynamische responsie van de ondersteuningsconstructie. Verschillende berekeningsmethoden voor deze demping zijn toegepast en geven bruikbare resultaten. Meer studie is vereist om de grootte van de aërodynamische demping nauwkeuriger te bepalen.

De frequentiedomein methode wordt op dit moment geïmplementeerd bij een offshore aannemer, terwijl verschillende andere bedrijven in de offshore windindustrie reeds hun interesse hebben getoond. 


\section{Curriculum vitae}

Jan van der Tempel was born in Enschede on January $18^{\text {th }} 1974$. He completed the Gymnasium at "Het Drachtster Lyceum" in 1992 upon which he started his study in Civil Engineering at the Delft University of Technology. He graduated in 2000 in the field of Offshore Engineering on his thesis titled: "Lifetime fatigue of offshore wind turbine support structures".

After his studies, he was employed by Royal Boskalis Westminster as a project engineer where his work focused on tendering for the installation of the Horns Rev offshore wind farm. He also worked in engineering and installation of several dredging and offshore projects.

Since the summer of 2001 he has been working as a $\mathrm{PhD}$ student at the Delft University of Technology in the Offshore Engineering and Wind Energy sections. During the five years of his $\mathrm{PhD}$ study he published over 20 scientific papers and coached over 50 students in BSc and MSc projects. Furthermore he was responsible for initiating and teaching the new student course in "Offshore Wind Farm Design".

He participated in a number of national and international research projects as task or project leader. He was also a co-author of project proposals, which have led to the financing of two new $\mathrm{PhD}$ positions within the Offshore Engineering section.

In the summer of 2002 he invented the Ampelmann together with a colleague. This patented system uses an inverted flight simulator on a ship to create a stationary transfer deck, even in high waves, to easily transfer personnel and equipment from the ship to the offshore wind turbine. The proof-of-concept tests of the system were completed in February 2005 and a prototype is currently being developed.

Next to over 30 articles in newspapers and magazines, the Ampelmann featured in two television shows and was demonstrated in the Dutch Houses of Parliament and at the EU in Brussels. For the Ampelmann, he was nominated and/or awarded the following recognitions:

- $\quad 1^{\text {st }}$ prize poster at the European Wind Energy Conference, London 2004

- UFD Ritsema van Eck prize for Excellent University Teamwork, 2005

- $\quad 1^{\text {st }}$ prize Writing a Business Plan, Netwerk Jonge Ondernemers, 2005

- Nominated for the Royal Shell Prize, 2005

- Winner of NewVenture round 1, 2005

- Short listed (final 6) for the Altran Foundation Award, 2006

He is currently employed by the DUT as a researcher/lecturer, mainly focusing on the development of the prototype of the Ampelmann.

In his spare time he reads, is a rugby referee and reconstructs houses. 